\title{
Dietary factors and low-grade inflammation in relation to overweight and obesity
}

Philip C. Calder ${ }^{1}$, Namanjeet Ahluwalia ${ }^{2}$, Fred Brouns ${ }^{3,21}$, Timo Buetler ${ }^{4,22}$, Karine Clement ${ }^{5}$,

Karen Cunningham ${ }^{6}$, Katherine Esposito ${ }^{7}$, Lena S. Jönsson ${ }^{8}$, Hubert Kolb ${ }^{9}$, Mirian Lansink ${ }^{10}$, Ascension Marcos ${ }^{11}$, Andrew Margioris ${ }^{12}$, Nathan Matusheski ${ }^{13}$, Herve Nordmann ${ }^{14}$, John O’Brien ${ }^{4}$, Giuseppe Pugliese ${ }^{15}$, Salwa Rizkalla ${ }^{5}$, Casper Schalkwijk ${ }^{16}$, Jaakko Tuomilehto ${ }^{17}$, Julia Wärnberg ${ }^{11,18}$, Bernhard Watzl ${ }^{19}$ and Brigitte M. Winklhofer-Roob ${ }^{20}$

1. School of Medicine, University of Southampton, Southampton SO16 6YD, UK

2. INSERM U557, University of Paris, 93017 Bobigny Cedex, France

3. Cargill R\&D Centre Europe, 1800 Vilvoorde, Belgium

4. Nestlé Research Centre, Vers-chez-les-Blanc, 1000 Lausanne, Switzerland

5. Department of Nutrition, INSERM U872, Research Center on Human Nutrition, Pitie Salpetriere Hospital, 75013 Paris, France

6. Coca-Cola Europe, Hammersmith, London W6 9HQ, UK

7. Division of Metabolic Diseases, University of Naples, 80138 Naples, Italy

8. ILSI Europe a.i.s.b.I., Avenue E. Mounier 83, Box 6, 1200 Brussels, Belgium

9. Research Group Immunobiology, Medical Faculty, University of Dusseldorf, 40225 Dusseldorf, Germany

10. Danone Research, Centre for Specialised Nutrition, 6700, CA, Wageningen, The Netherlands

11. Department of Metabolism and Nutrition, CSIC, Spanish National Research Council, 28040 Madrid, Spain

12. School of Medicine, University of Crete, 71409 Heraklion, Greece

13. Nutrition Research, Kraft Foods, Inc., Glenview IL 60025, USA

14. Ajinomoto Europe, 75817 Paris, France

15. Department of Clinical and Molecular Medicine, "La Sapienza" University of Rome, 00161 Rome, Italy

16. Internal Medicine, University of Maastricht, 6202 AZ Maastricht, The Netherlands

17. University of Helsinki, 00014 Helsinki, Finland

18. Department of Preventive Medicine and Public Health, University of Navarra, 31080- Pamplona, Spain

19. Max-Rubner Institut, Federal Research Centre for Nutrition and Food, 76131 Karlsruhe, Germany

20. Institute of Molecular Biosciences, Human Nutrition and Metabolism Research and Training Center, Karl-Franzens University of Graz, 8010 Graz, Austria

21. Department of Nutrition, Maastricht University Medical Centre, 6200 MD Maastricht, The Netherlands

22. XeRR, Institute of Pharmacology and Toxicology, University of Zürich, 8057, 8057 Zürich, Switzerland

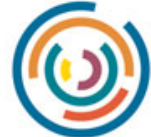

$\mid \mathrm{LSI}$

Europe

International Life

Sciences Institute
Commissioned by the

ILSI Europe Metabolic Syndrome and Diabetes Task Force

Correspondence: ILSI Europe a.i.s.b.I. - Avenue E. Mounier 83, Box 6 - 1200 Brussels, Belgium, fax +32 276200 44, email publications@ilsieurope.be 


\section{British Journal of Nutrition \\ Volume 106, 2011 ISSN: 0007-1145}

Publishing, Production, Marketing, and Subscription Sales Office:

Cambridge University Press

The Edinburgh Building

Shaftesbury Road

Cambridge CB2 8RU, UK

For Customers in North America:

Cambridge University Press

Journals Fullfillment Department

100 Brook Hill Drive

West Nyack

New York 10994-2133

USA

Publisher: Katy Christomanou

Special sales and supplements:

This Journal accepts relevant advertisements and inserts. We also provide bulk reprints of suitable papers to meet teaching or promotional requirements. The journal also publishes supplements on behalf of academic and corporate collaborators. Please contact Katy Christomanou at the Cambridge address for further details. E-mail: kchristomanou@cambridge.org

Subscription information:

British Journal of Nutrition is an international journal published by Cambridge University Press on behalf of The Nutrition Society. The twelve issues starting January 2011 comprise Volume 105, the twelve issues starting July 2011 comprise Volume 106.

Annual subscription rates:

Volumes 105/106 (24 issues):

Internet/print package $£ 1021 / \$ 1990 / € 1635$

Internet only: $£ 835 / \$ 1630 / € 1334$

Print only: £973/\$1895/€1580

Any supplements to this journal published in the course of the annual volume are normally supplied to subscribers at no extra charge.

Back volumes are available. Please contact Cambridge University Press for further information.

Claims for non-receipt of journal issues will be considered on their merit and only if the claim is received within six months of publication. Replacement copies supplied after this date will be chargeable.
US POSTMASTERS: please send address corrections to British Journal of Nutrition, Cambridge University Press, 100 Brook Hill Drive, West Nyack, New York 10994-2133.

Directions to Contributors are available from the Society at the address below or can be found on the Society's website at http://www.nutritionsociety.org (an abbreviated Notes for Authors can be found inside the back cover).

Offprints: The author (or main author) of an accepted paper will receive a copy of the PDF file and a voucher copy of the issue in which their paper has been published. There will be an option to purchase paper offprints, these should be ordered at proof stage. No page charges are levied by this journal.

Copyright: As of 1 July 2000 the copyright of all articles submitted to British Journal of Nutrition are retained by the authors or their institutions. For articles prior to this date permission for reproduction of any part of the journal (text, figures, tables or other matter) in any form (on paper, microfiche or electronically) should be sought directly from the Society, at: The Publications Office, The Nutrition Society, 10 Cambridge Court, 210 Shepherds Bush Road, Hammersmith, London W6 7NJ, UK.

Disclaimer: The information contained herein including any expression of opinion and any projection or forecast, has been obtained from or is based upon sources believed by us to be reliable, but is not guaranteed as to accuracy or completeness. The information is supplied without obligation and on the understanding that any person who acts upon it or otherwise changes his/her position in reliance thereon does so entirely at his/her own risk. Neither the Society nor Cambridge University Press accepts responsibility for any trade advertisement included in this publication.

This journal is printed on acid-free paper from renewable sources. Printed in the UK by Bell \& Bain Ltd., Glasgow.

This journal issue has been printed on FSC-certified paper and cover board. FSC is an independent, nongovernmental, not-for-profit organization established to promote the responsible management of the world's forests. Please see www.fsc.org for information.

British Journal of Nutrition is covered in Current Contents ${ }^{\circledR} /$ Agriculture, Biology \& Environmental Sciences, SciSearch ${ }^{\circledR}$, Research Alert ${ }^{\circledR}$, Current Contents ${ }^{\circledR}$ Life Sciences, Index Medicus ${ }^{\circledR}$ (MEDLINE ${ }^{\circledR}$ ), AGRICOLA $^{\circledR}$, CAB Abstracts ${ }^{\text {TM }}$, Global Health, BIOSIS ${ }^{\circledR}$ Database, EMBASE/Excerpta Medica and Elsevier BIOBẢSE/Current Awareness in Biological Sciences, CINAHL, and Chemical Abstracts Service. 
Vol. 106 Supplement No. S3 December $2011 \quad$ British Journal of Nutrition

\title{
Table of Contents
}

$\begin{array}{lr}\text { Preamble } & \text { S5 }\end{array}$

$\begin{array}{lr}\text { Concept and markers of low-grade inflammation } & \text { S5-S11 }\end{array}$

$\begin{array}{lr}\text { Chronic low-grade inflammation and insulin resistance } & \text { S11-S13 }\end{array}$

$\begin{array}{lr}\text { Postprandial inflammatory response } & \text { S13-S14 }\end{array}$

$\begin{array}{lr}\text { Ageing and low-grade inflammation } & \text { S14-S15 }\end{array}$

$\begin{array}{lr}\text { Exercise and low-grade inflammation } & \text { S15-S18 }\end{array}$

A consideration of different approaches to identify relationships between diet and its
components and markers of chronic low-grade inflammation

$\begin{array}{lr}\text { Dietary patterns and low-grade inflammation } & \text { S18-S28 }\end{array}$

Process-related compounds: advanced glycation end products and advanced lipoperoxidation end products

$\begin{array}{lr}\text { Micronutrients and phytochemicals } & \text { S46-S52 }\end{array}$

$\begin{array}{lr}\text { Other factors } & \text { S52-S53 } \\ \text { Sum } & \text { S53-S54 }\end{array}$

$\begin{array}{lr}\text { Summary, conclusions and research gaps } & \text { S53-S54 }\end{array}$

$\begin{array}{lr}\text { Acknowledgements } & \text { S54-S55 }\end{array}$

(Received 20 January 2011 - Revised 8 August 2011 - Accepted 7 September 2011)

Key words: Inflammation: Cytokines: Adipose: Obesity: Diet

Correspondence: ILSI Europe a.i.s.b.1., Avenue E. Mounier 83, Box 6 - 1200 Brussels, Belgium, fax +32 276200 44, email publications@ilsieurope.be

\begin{abstract}
Abbreviations: AGE, advanced glycation end products; AGE-R, advanced glycation end product receptor; AHEI, alternate healthy eating index; ALE, advanced lipoxidation end products; CCL, chemokine (C-C motif) ligand; CCR, CC chemokine receptor; $\mathrm{CFU}$, colony forming units; CLA, conjugated linoleic acids; CRP, C-reactive protein; DQI, Diet Quality Index; DQI-R, revised Diet Quality Index; GI, glycaemic index; GL, glycaemic load; HEI, healthy eating index; IFN, interferon; IL-1ra, IL-1 receptor antagonist; IRS, insulin receptor substrate; LPS, lipopolysaccharide; MAPK, mitogen-activated protein kinase; MCP, monocyte chemoattractant protein; MIF, macrophage migration inhibitory factor; MIP, macrophage inflammatory protein; MRP, Maillard reaction products; NHANES, National Health and Nutrition Examination Survey; PAI-1, plasminogen activator inhibitor 1; Q, quintile; RAGE, receptor for advanced glycation end products; RANTES, regulated on activation, normal $\mathrm{T}$ expressed and secreted; ROS, reactive oxygen species; SAA, serum amyloid A; sE-selectin, soluble E-selectin; sICAM-1, soluble intercellular adhesion molecule-1; sP-selectin, soluble P-selectin; STAMP, six-transmembrane protein of prostate; sTNFR, soluble receptors of TNF; sVCAM-1, soluble vascular cell adhesion molecule-1; TGF, transforming growth factor; TLR, Toll-like receptors.
\end{abstract}

(C) ILSI Europe [2011] 
Low-grade inflammation is a characteristic of the obese state, and adipose tissue releases many inflammatory mediators. The source of these mediators within adipose tissue is not clear, but infiltrating macrophages seem to be especially important, although adipocytes themselves play a role. Obese people have higher circulating concentrations of many inflammatory markers than lean people do, and these are believed to play a role in causing insulin resistance and other metabolic disturbances. Blood concentrations of inflammatory markers are lowered following weight loss. In the hours following the consumption of a meal, there is an elevation in the concentrations of inflammatory mediators in the bloodstream, which is exaggerated in obese subjects and in type 2 diabetics. Both high-glucose and high-fat meals may induce postprandial inflammation, and this is exaggerated by a high meal content of advanced glycation end products (AGE) and partly ablated by inclusion of certain antioxidants or antioxidant-containing foods within the meal. Healthy eating patterns are associated with lower circulating concentrations of inflammatory markers. Among the components of a healthy diet, whole grains, vegetables and fruits, and fish are all associated with lower inflammation. AGE are associated with enhanced oxidative stress and inflammation. SFA and trans-MUFA are pro-inflammatory, while PUFA, especially long-chain $n$-3 PUFA, are anti-inflammatory. Hyperglycaemia induces both postprandial and chronic low-grade inflammation. Vitamin C, vitamin E and carotenoids decrease the circulating concentrations of inflammatory markers. Potential mechanisms are described and research gaps, which limit our understanding of the interaction between diet and postprandial and chronic low-grade inflammation, are identified.

\section{Preamble}

Inflammation is a normal defence mechanism that protects the host from infection and other insults; it initiates pathogen killing as well as tissue repair processes and helps to restore homeostasis at infected or damaged sites. It is typified by redness, swelling, heat, pain and loss of function, and involves interactions among many cell types and the production of, and responses to, a number of chemical mediators. Self-regulation of the inflammatory response involves the activation of negative feedback mechanisms such as the secretion of anti-inflammatory cytokines, inhibition of proinflammatory signalling cascades, shedding of receptors for inflammatory mediators and activation of regulatory cells. As such, and controlled properly, regulated inflammatory responses are essential to remain healthy and maintain homeostasis. Pathological inflammation involves a loss of tolerance and/or of regulatory processes. Where this becomes excessive, irreparable damage to host tissues and disease can occur $^{(1)}$. Such diseases are characterised by markedly elevated concentrations of inflammatory markers and of activated inflammatory cells at the site of tissue damage and in the systemic circulation. While the existence of inflammatory diseases has been long recognised, it is only more recently that the condition of chronic low-grade inflammation has received attention, particularly in relation to obesity, the metabolic syndrome and CVD. Chronic low-grade inflammation is characterised by raised concentrations of inflammatory markers in the systemic circulation. This article sets out to explain the nature of chronic low-grade inflammation in the context of overweight and obesity, and to describe the factors that might influence it, in particular those related to diet. The literature in the areas of adipose tissue, obesity and inflammation, and dietary components and inflammation is vast, and it is not possible to mention all studies here. In particular, the review of diet and its components and inflammation is not exhaustive, although the main studies of relevance are included.

\section{Concept and markers of low-grade inflammation}

\section{Obesity and low-grade inflammation}

The concept of systemic, chronic, but low-grade inflammation as a risk factor for the metabolic syndrome and for type 2 diabetes is based on the observation of elevated blood levels of inflammation-associated markers in people with incident type 2 diabetes or with the metabolic syndrome ${ }^{(2,3)}$. The up-regulation of systemic indicators of inflammation such as leucocyte count, and serum and plasma concentrations of acute-phase proteins, pro-inflammatory cytokines, chemokines, soluble adhesion molecules and prothrombotic mediators is modest, usually less than 2-fold above what is observed in controls. Diagnostic criteria for low-grade inflammation have not been precisely defined, but the phenotype per se is not disputed.

Systemic concentrations of pro-inflammatory mediators are higher in obese $\left(\mathrm{BMI}>30 \mathrm{~kg} / \mathrm{m}^{2}\right)$ than in normal-weight persons ${ }^{(4,5)}$. Serum or plasma concentrations of TNF- $\alpha$ or IL- 6 in healthy adults are typically $0 \cdot 01-2 \mathrm{pmol} / \mathrm{l}^{(6)}$. Other inflammatory mediators, such as monocyte chemoattractant protein (MCP)-1, interferon (IFN)- $\boldsymbol{\gamma}$-induced protein-10 and IL-18, may reach mean concentrations of $10 \mathrm{pmol} / 1$; macrophage migration inhibitory factor (MIF) and regulated on activation, normal $\mathrm{T}$ expressed and secreted (RANTES) concentrations may get close to the nanomolar range; and $\mathrm{C}$-reactive protein (CRP) concentration is often above $10 \mathrm{nmol} / \mathrm{l}$. The variation in concentrations of most mediators among non-obese or obese individuals is at least 10-fold. Hence, there is a substantial overlap between non-obese and obese persons. However, there is a positive relationship between BMI and other measures of obesity such as waist circumference and circulating concentrations of CRP and other inflammatory markers ${ }^{(7)}$

A mechanistic link between obesity and low-grade inflammation was first proposed by Hotamisligil et al. ${ }^{(8)}$ who showed that white adipose tissue synthesises and releases the pro-inflammatory cytokine TNF- $\alpha$. The expression of TNF- $\alpha$ is elevated in adipocytes of obese and insulin-resistant mice, while insulin sensitivity is improved following administration of anti-TNF- $\alpha$ antibodies. Based on these data, it was suggested that adipose tissue plays an important immune role and may be a major 
source of pro-inflammatory mediators which initiate the development of chronic inflammation, insulin resistance and atherosclerosis, all of which are characteristics of the metabolic dysregulation associated with obesity. The discovery of leptin modified the view of adipose tissue as being an 'inert' energy store to being the largest endocrine gland in the body. Leptin is produced and secreted by white adipose tissue. The discovery of leptin introduced the concept of 'adipocytokines' or 'adipokines', substances produced by adipose tissue and which circulate in the bloodstream, so exerting systemic effects as hormones ${ }^{(9)}$. Some adipokines are produced within adipose tissue exclusively by adipocytes (e.g. leptin, adiponectin, serum amyloid A (SAA)), while others are produced by both adipocytes and other cell types of the non-adipocyte fraction of adipose tissue. It is now recognised that macrophages accumulate in the adipose tissue in obesity ${ }^{(10,11)}$ (Fig. 1) and that these may represent major contributors to the production of adipokines $^{(12,13)}$.

\section{Adipose tissue as a source of inflammatory mediators}

Adipose tissue expresses and secretes into the systemic circulation a growing list of hormones, inflammatory mediators and immune system effectors. The products of adipose tissue can be categorised into several groups (Table 1):

(1) Hormones: many of the hormones produced by adipose tissue affect the immune system and insulin sensitivity. Leptin appears to be pro-inflammatory ${ }^{(14)}$, while adiponectin appears to be anti-inflammatory and insulin-sensitising ${ }^{(15)}$. Similarly, visfatin ${ }^{(16)}$ and resistin ${ }^{(17)}$ contribute to the development of insulin resistance, while omentin $^{(18,19)}$ appears to be an insulin sensitiser. It should be noted that most studies identifying the roles of these hormones have been performed in rodents and the immune and insulin-sensitising effects of these hormones in humans remain unclear ${ }^{(20,21)}$.

(2) Acute-phase proteins: these proteins are secreted in the acute phase of inflammation and include plasminogen activator inhibitor 1 (PAI- 1$)^{(22)}$, pentraxine-3, lipocalin $24 \mathrm{p} 3$, haptoglobin, $\mathrm{SAA}^{(23)}$ and $\alpha 1$-glycoprotein.

(3) Cytokines: these are the classic peptide mediators of inflammation and include IL-1, IL-1 receptor antagonist $(\mathrm{IL}-1 \mathrm{ra})^{(24-26)}, \mathrm{IL}-6, \mathrm{IL}-7, \mathrm{IL}-18^{(27-30)}, \mathrm{IL}-10^{(31,32)}, \mathrm{MIF}^{(33)}$ and TNF- $\alpha$.

(4) Chemokines: these include IL- $8^{(34,35)}, \mathrm{MCP}-1,-3,-4$, RANTES (now known as chemokine (C-C motif) ligand (CCL) 5), angiopoietin, metallothioneins, macrophage inflammatory protein (MIP)- $1 \alpha$ and $-1 \beta$ (now known as CCL3 and CCL 4 , respectively) ${ }^{(36)}$, and induced protein- $10^{(37)}$

(5) Growth factors: transforming growth factor (TGF)- $\beta^{(38)}$.

(6) Components of the alternative complement system: adipsin and factors C2, C3, C4, C7, B and D (these are expressed more highly in omental compared with subcutaneous adipose tissue ${ }^{(39,40)}$ ).

(7) Retinol-binding protein 4 which is linked with insulin resistance $^{(41,42)}$, although its precise role has been debated ${ }^{(43)}$.

\section{Adipose tissue distribution and its impact on inflammation}

Obesity is characterised by an expansion of the mass of adipose tissue and dramatic changes in its distribution in the body. Simplistically speaking, accumulation of adipose tissue in the thorax and abdomen (variously termed abdominal, central, visceral, splanchnic or android obesity) results in an increased risk for diabetes and atherosclerosis, while excess adipose tissue in the lower part of the body (termed gynoid obesity) does not appear to be associated with major metabolic consequences ${ }^{(44,45)}$. The increase in abdominal fat mass is associated with a chronic elevation of the circulating concentrations of inflammatory mediators including several acute-phase inflammatory proteins such as $\mathrm{CRP}^{(46,47)}$, proand anti-inflammatory cytokines, adhesion molecules and pro-thrombotic molecules ${ }^{(22,47-49)}$. It should be noted that the liver and the lymphoid organs are usually the major production sites of these inflammatory mediators but in obesity, adipose tissue becomes a major producer resulting in a chronic and constant local and systemic inflammatory milieu (Table 2). Abdominal obesity is a risk factor for type 2 diabetes, hypertension, dyslipidaemia and $\mathrm{CVD}^{(50)}$, and also probably obesityassociated hepatic diseases (non-alcoholic fatty liver disease and non-alcoholic steatohepatitis). Glucose intolerance is significantly more common in subjects with abdominal obesity compared with those with fat mass accumulation in their lower part of the body. Plasma TAG concentrations are also significantly more elevated in individuals with abdominal obesity. It appears that the anatomical localisation of adipose tissue is of paramount importance in relation to its physiological function, i.e. handling of lipids (lipogenesis, lipolytic activity), expression of multiple genes, and response to insulin, catecholamines, sex hormones and cortisol ${ }^{(51)}$. In addition, the profile of adipokines produced is dissimilar between the subcutaneous and abdominal adipose tissues. Thus, leptin is preferentially expressed and secreted by subcutaneous adipose tissue ${ }^{(52)}$, while the expression of adiponectin, visfatin, omentin, resistin, PAI-1, IL-8, IL-7, IL- $1 \alpha$, MCP-1, TGF- $\beta$, growth-related oncogen$\alpha$, CCL 5 and MIP-1 $\beta$ is higher in abdominal fat. In contrast to such distributions, there are reports that IL- 6 and TNF- $\alpha$ seem to be equally synthesised by the different $\operatorname{sites}^{(28,36,53-59)}$. It is important to mention that in severe obesity, the part played by the abdominal or the very abundant subcutaneous adipose tissue in the systemic delivery of inflammatory mediators is still not well understood. Nevertheless, the distinct profile of adipokine secretion between the abdominal and subcutaneous adipose tissues probably contributes to the increased risk of metabolic and cardiovascular complications and to the development of other complications such as hepatic steatosis and non-alcoholic steatohepatitis in obese individuals. Finally, other adipose tissue depots in so-called 'ectopic sites', such as within the liver, heart or skeletal muscle, may contribute to the production of inflammatory mediators in the absence of obesity. In this regard, the local production of the inflammatory molecules by adipose tissue within the heart may be important; the amount of this tissue and its proximity to the coronary vessels could contribute to the development of coronary pathologies ${ }^{(60,61)}$. 


\section{Cell populations of adipose tissue}

Adipose tissue is a heterogeneous tissue composed of several cell types: mature adipocytes, pre-adipocytes, fibroblasts, endothelial cells, mast cells, granulocytes, lymphocytes and macrophages. Cells within adipose tissue, apart from mature adipocytes, are collectively termed the stroma-vascular fraction. The various cell types have not been precisely characterised, nor has the relative change of their quantitative contributions to the tissue in obesity. Because of the heterogeneity of cells in the adipose tissue, the cellular source of the inflammatory factors secreted by the tissue into the systemic circulation remains unknown. In vitro studies have demonstrated that mature adipocytes express inflammatory factors such as TNF- $\alpha^{(56)}$. SAA is overexpressed and secreted in abundance by isolated adipocytes from obese subjects, as is leptin, while secretion of adiponectin is suppressed. SAA and leptin production by adipose tissue depends on adipocyte size $^{(18,19,23,62)}$. Adipocyte size also influences the expression of other inflammatory mediators as demonstrated by fractionation studies of adipocytes coupled with studies of gene expression profiles ${ }^{(62)}$. Adipocyte size, for example, determines the production of IL-6, IL-8, MCP-1 and granulocyte colony-stimulating factor ${ }^{(29)}$. Although adipocyte hypertrophy precedes the development of type 2 diabetes $^{(63)}$, a growing number of studies indicate that the principal site of production of inflammatory mediators appears to be the stroma-vascular fraction ${ }^{(36,64-67)}$. More recent studies in mice have suggested that the infiltration of obese adipose tissue by macrophages is accompanied or even preceded by an influx of T-lymphocytes $^{(68-71)}$ and that T-cells have a key early role ${ }^{(69)}$. Early work indicated the presence of CD3-positive T-lymphocytes in human adipose tissue ${ }^{(72)}$, and more recent studies have shown high numbers of T-cells in the adipose tissue of

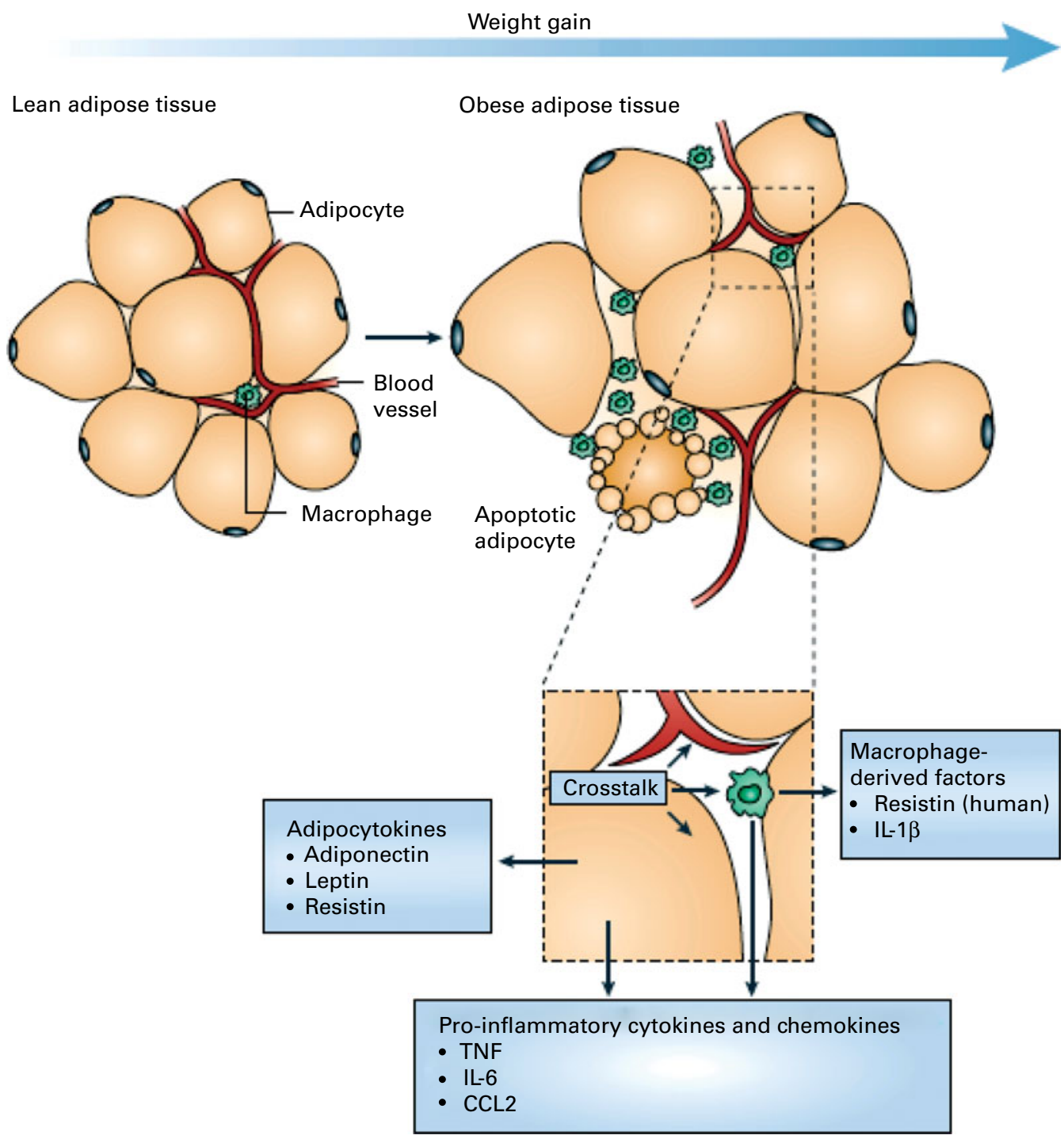

Fig. 1. Schematic representation of the interaction between adipocytes and macrophages showing some of the molecules released. Expansion of adipose tissue during weight gain leads to the recruitment of macrophages through various signals (e.g. chemokines such as chemokine (C-C motif) ligand 2 (CCL2)) released by adipocytes. Macrophages accumulate around apoptotic adipocytes. Mediators synthesised by adipocytes and resident macrophages contribute to local and systemic inflammation. Reproduced with permission from Tilg \& Moschen ${ }^{(10)}$. 
Table 1. Cytokines expressed or secreted by human adipose tissue

\begin{tabular}{ll}
\hline Family & Example(s) \\
\hline Chemokines & MCP-1 (known as CCL2), MCP-3, \\
& MCP-4, RANTES (known as CCL5), \\
& MIP-1 $\alpha$ (known as CCL3) \\
IL & IL-6, IL-8 (acts as a chemokine), IL-1 ra, IL-10, IL-18 \\
Interferons & IP-10 \\
TNF & TNF- $\alpha$ \\
Growth factors & Vascular endothelial growth factor, TGF- $\beta$, \\
& hepatocyte growth factor \\
Others & Leptin
\end{tabular}

MCP, monocyte chemoattractant protein; $\mathrm{CCL}$, chemokine (C-C motif) ligand; RANTES, regulated on activation, normal $\mathrm{T}$ expressed and secreted; MIP macrophage inflammatory protein; IL-1 ra, IL-1 receptor antagonist; IP, interferon$\gamma$-induced protein; TGF, transforming growth factor.

diet-induced obese insulin-resistant mice $^{(71)}$. Furthermore, Wu et al. $^{(71)}$ demonstrated the presence of CD3-positive T-lymphocytes in human adipose tissue and described the expression of RANTES, a T-cell-specific chemokine, and its respective receptor CCR5 in the visceral adipose tissue of morbidly obese patients. A recent study in mice reported mainly CD8-positive lymphocyte infiltration in hypoxic areas within the adipose tissue ${ }^{(70)}$. Most recently, it has been shown that pro-inflammatory T-lymphocytes are present in visceral adipose tissue and may contribute to local inflammatory cell activation before the appearance of macrophages, suggesting that these cells could play an important role in the initiation and perpetuation of adipose tissue inflammation as well as the development of insulin resistance ${ }^{(69)}$.

It has been proposed that macrophages and mature adipocytes are derived from the same precursor cells and show close gene expression profiles including the Toll-like receptors (TLR). Pre-adipocytes exert 'macrophage-like' effects when exposed to strong pro-inflammatory environments ${ }^{(73,74)}$ It should be noted, however, that the vast majority of the macrophage infiltration in adipose tissue in obesity originates most probably from the circulation. In obese subjects, these macrophages typically aggregate in crowns around apoptotic adipocytes $^{(31)}$ (Figs. 1 and 2). Although these macrophages express activation markers, they could be pro- or antiinflammatory depending on the degree of obesity and its evolution as suggested by studies in mice showing that weight gain is accompanied by transformation from a macrophagic M2 (anti-inflammatory) phenotype towards an M1 (proinflammatory) profile ${ }^{(75)}$ (Fig. 3) ${ }^{(76)}$. Consequently, secretion profiles of the adipose tissue can change depending on the phenotype of the cell population infiltrating it during the different stages of obesity (initiation, aggravation, maintenance and weight loss; Fig. 4) ${ }^{(77)}$.

Adipose tissue macrophages may contribute to the maintenance of the low-grade inflammatory state linked to obesity ${ }^{(36)}$. Factors that induce the infiltration and activation of macrophages in the adipose tissue are probably multifactorial. Paracrine, autocrine and endocrine signals, as well as mechanical modifications (hypertrophy and adipocyte hyperplasia), could play a role in this phenomenon. Many adipokines synthesised by the adipose tissue are candidates to attract inflammatory cells. In vitro studies have suggested that leptin itself (at supra-physiological levels) induces adhesion proteins, hence facilitating the migration of monocytes ${ }^{(78)}$. Conversely, adiponectin may inhibit this process ${ }^{(79)}$. Very little is known about the role of selectins, integrins and elements of adhesion to the extracellular matrix, in the process of macrophage attraction to the adipose tissue. Gene expression studies with human adipose tissue have demonstrated that the levels of expression of MCP-1, colony-stimulating factor-3 and the urokinase plasminogen activator CD87 increase significantly in the adipose tissue of subjects with morbid obesity ${ }^{(31)}$. MCP-1 is a strong chemoattractant and it acts via its receptor CCR2. MCP-1 is synthesised predominantly by macrophages and endothelial cells and, to a lesser extent, by adipocytes. In one study, CCR 2 gene knockout mice showed a reduction of macrophage infiltration in the adipose tissue and improvement of insulin sensitivity ${ }^{(80)}$. This led to the suggestion that MCP-1 and its receptor CCR2 are major players in the macrophage accumulation within the adipose tissue $^{(80)}$. However, contradictory data suggest that MCP-1 might not be such a crucial candidate ${ }^{(81)}$. The role of MCP-1 in the macrophage accumulation in human obesity needs to be established. Other candidate molecules and other mechanisms continue to be explored. Local hypoxia could also play an important role in the attraction and retention of macrophages within the adipose tissue ${ }^{(82)}$. Hypoxia-inducible factor- $1 \alpha$, a transcription factor normally induced by hypoxia, is overexpressed in the subcutaneous adipose tissue of obese subjects and its expression is decreased during weight reduction $^{(31)}$. Tissue hypoxia induces macrophage attraction into solid tumours as well as into atherosclerotic plaques. Adipose tissue of obese subjects could be hypoxic in some areas and a local expression of chemokines could be induced. It should be noted that leptin, which possesses indirect chemoattractant properties, is induced by hypoxia ${ }^{(83)}$.

It is generally considered that macrophage accumulation in adipose tissue is detrimental. However, macrophage accumulation could be related to an adaptation process associated with the augmentation of fat mass, and macrophage accumulation could be necessary for the upkeep of the tissue and perhaps to limit its expansion. It appears that macrophage aggregates within the adipose tissue are localised around apoptotic adipocytes, suggesting that one of their functions is to clean up the debris of dying and dead cells ${ }^{(84)}$. In addition to their role in cleaning up the old cells, the accumulation of macrophages may also be useful for the formation of new vessels, particularly at the site of inflammation and in ischaemic zones when adipose tissue grows ${ }^{(85)}$. Macrophages also control fat mass growth and modify the biology of adipocytes and pre-adipocytes in a paracrine manner. The specific effects of TNF- $\alpha$ and IL- 6 on different adipocyte functions (increased lipolysis, modification of adipocyte secretion patterns and induction of adipocyte insulin resistance) have been shown ${ }^{(86)}$. In the presence of a medium derived from human macrophages, human pre-adipocytes showed a drastic change in their phenotype, acquiring a pro-inflammatory phenotype and secreting significant amounts of IL- 6 and IL-8, and they grew well ${ }^{(87)}$, but differentiated poorly ${ }^{(87,88)}$. 
Table 2. Modification of circulating inflammatory marker concentrations in relation to obesity and weight loss

\begin{tabular}{|c|c|c|}
\hline & Effect of obesity & Effect of weight loss \\
\hline \multicolumn{3}{|l|}{ Acute-phase proteins } \\
\hline C-reactive protein & Increase & Decrease \\
\hline Fibrinogen & Increase & Decrease \\
\hline Orosomucoid & Increase & Decrease \\
\hline Haptoglobin & Increase & Decrease \\
\hline Serum amyloid $\mathrm{A}$ & Increase & Decrease \\
\hline \multicolumn{3}{|l|}{ Cytokines } \\
\hline IL-6 & Increase & Decrease \\
\hline IL-8 & Increase & Decrease \\
\hline IL-18 & Increase & Decrease \\
\hline IL-10 & Increase & Decrease \\
\hline IL-1 receptor antagonist & Increase & Decrease \\
\hline TNF- $\alpha$ & Increase & None or decrease \\
\hline Monocyte chemoattractant protein-1 & Increase & Decrease \\
\hline Monocyte chemoattractant protein- 4 & Increase & Not known \\
\hline Macrophage migration inhibitory factor & Increase & Decrease \\
\hline \multicolumn{3}{|l|}{ Other adipokines } \\
\hline Leptin & Increase & Decrease \\
\hline Visfatin & Increase & Decrease \\
\hline Resistin & Decrease & None or increase \\
\hline Adiponectin & Decrease & Increase \\
\hline Omentin & Decrease & Not known \\
\hline \multicolumn{3}{|c|}{ Adhesion proteins/extracellular matrix remodelling proteins/prothrombotics } \\
\hline Matrix metalloproteinase 9 & Increase & Decrease \\
\hline Soluble intercellular adhesion molecule-1 & Increase & Decrease \\
\hline Soluble vascular cell adhesion molecule-1 & Increase & Decrease \\
\hline Soluble E-selectin & Increase & Decrease \\
\hline Hepatocyte growth factor & Increase & Not known \\
\hline Plasminogen activator inhibitor 1 & Increase & Decrease \\
\hline Cathepsin S & Increase & Decrease \\
\hline
\end{tabular}

These cellular alterations are induced in co-cultures without direct cellular contact, suggesting the key role of soluble factors, although it cannot be excluded that a direct cell-cell interaction also plays a role in modifying pre-adipocyte or adipocyte biology. The mature adipocyte also endures profound modifications of its biology in culture systems with a medium from activated macrophages. Other than the proinflammatory state, increased lipolysis and resistance to insulin have been observed ${ }^{(89,90)}$. TNF- $\alpha$ has been proposed to mediate these effects. From the molecular point of view, the $\mathrm{NF}-\kappa \mathrm{B}$ pathway, implicated in the primary regulation of inflammatory responses ${ }^{(91-93)}$ (Fig. 5) ${ }^{(94)}$, is induced in the pre-adipocyte $^{(87)}$ and in the adipocyte in the presence of a medium from macrophages ${ }^{(95)}$. The NF- $\mathrm{kB}$ pathway is also brought into play in macrophages in contact with a medium from adipocytes. TLR appear to be important players which lead to the induction or suppression of genes orchestrating the inflammatory response. TLR- 4 is the bacterial lipopolysaccharide (LPS) receptor, but data have shown that the NEFA produced by adipocytes after adrenergic stimulation are also strong inducers of the TLR-4/NF-кB system ${ }^{(95)}$ (Fig. 5). TLR-4 is expressed by adipocytes and is overexpressed during obesity ${ }^{(96)}$. TLR- 4 knockout mice are protected from insulin resistance induced by lipid infusions ${ }^{(97)}$.

Based on these different studies, a dual effect of macrophages of the adipose tissue could be expected: first, a local 'beneficial' effect in clearing out old adipocytes, and in the control and of the development of fat mass and second, a deleterious local and systemic effect via the increase in the production and secretion of adipokines, promoting the progression of complications of obesity and the induction of insulin resistance.

\section{Adipokines and chronic low-grade inflammation}

Adipokines and the complications of obesity. Inflammatory molecules are likely candidates exerting a molecular link between the adipose tissue and the metabolic, cardiovascular, hepatic and thrombotic complications, and certain cancer types occurring in conjunction with or as a consequence of human obesity. A myriad of candidate adipokines are proposed to play this role ${ }^{(98-100)}$. In the cardiovascular field, they can be considered as risk factors, and even directly play a pathophysiological role favouring the initiation and progression of atherosclerosis. Relationships between abnormalities of cardiac function in obese subjects, the accumulation of abdominal fat and low-grade inflammation have been suggested ${ }^{(101,102)}$. Among the candidates secreted by the adipose tissue, the increase in IL- 6 , IL- 8 and MCP-1 and the decrease in adiponectin are considered to be particularly important ${ }^{(101,102)}$. The studies of the pathophysiological links between adipokines and cardiovascular health can be illustrated by the analysis of the effects of adiponectin in rodents. Overexpression of adiponectin results in diminished size of the lesions observed following acute ischaemic myocardial infarction, increased angiogenic properties and reduced size of atheromatous plaques in the genetically predisposed apoE ${ }^{-/-}$mouse ${ }^{(103)}$. 

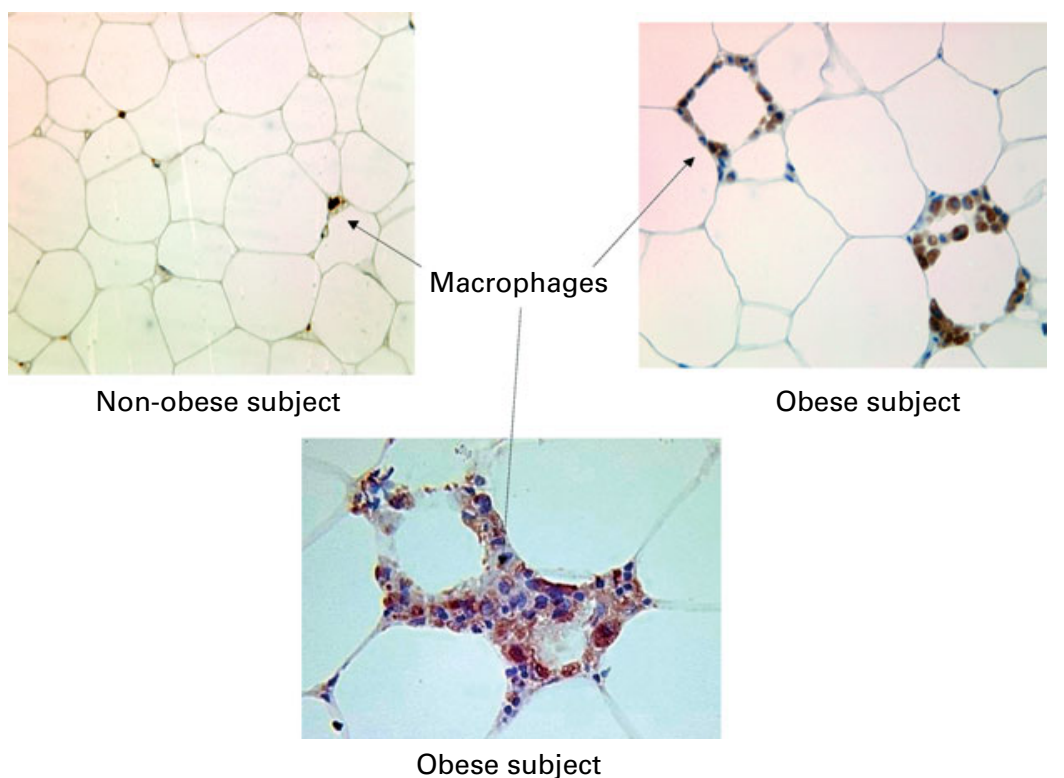

Fig. 2. Adipose tissue from non-obese and obese human subjects showing macrophage infiltration. Macrophages are stained with HAM56 antibody. Reproduced with permission from Cancello et al. ${ }^{(65)}$.

Several inflammatory mediators produced by adipose tissue, such as CCL5, IL-1 $\beta$ and IL-8, as well as markers of oxidative stress, are increased in diabetic or glucose-intolerant patients, and the amelioration of hyperglycaemia by insulin therapy reduces circulating concentrations of these molecules ${ }^{(104,105)}$. The increase in the concentrations of TNF- $\alpha$, IL- 6 , IL-1 $\beta$, IL-8, resistin and many other factors produced by macrophage activation could contribute to the deterioration of insulin sensitivity (Fig. 6) ${ }^{(106)}$. The precise relationship between the importance of macrophage and T-cell accumulation in adipose tissue depots, adipokine secretion and the modifications of insulin sensitivity needs to be further addressed in humans.

Macrophage accumulation is more abundant in the abdominal adipose tissue ${ }^{(65)}$ than in the subcutaneous tissue, and this could explain some of the risks associated with the accumulation of intra-abdominal fat. For example, a relationship between the increase in macrophages in the abdominal adipose tissue and hepatic inflammation and fibrosis has been reported $^{(65)}$. In another study, the expression of the MCP-1 and colony-stimulating factor-1 genes and proteins was associated with macrophage accumulation in obese subjects ${ }^{(107)}$. Since the abdominal adipose tissue is partly drained by the portal system, it cannot be excluded that some adipokines, together with high NEFA fluxes and hormones delivered by the adipose tissue, could contribute to the alteration of hepatic function observed in obese subjects, the mechanism of which needs to be better dissected.

Adipokines and weight loss. Even modest weight reduction improves the metabolic and cardiovascular risks associated with human obesity. Measures of endothelial activation also improve after weight reduction ${ }^{(108-110)}$. Many studies have shown that weight loss induced by a decrease in energy intake, and sometimes an increase in exercise, reduces systemic inflammation. A reduction in concentrations of numerous inflammatory molecules and endothelial risk factors, and an increase in adiponectin concentration have been observed during weight-loss programmes, and these are sometimes associated with improvement of insulin sensitivity ${ }^{(111)}$. Such changes have been described for $\mathrm{CRP}^{(112)}$, IL-6 ${ }^{(113)}$, IL-18 ${ }^{(114)}$, IL-1ra ${ }^{(26)}$, PAI-1 ${ }^{(115)}$, SAA $^{(23,116)}$, cathepsin $\mathrm{S}^{(117)}$, matrix metalloproteinase- $9^{(118)}$, soluble adhesion molecules (soluble intercellular adhesion molecule-1 (sICAM-1), soluble vascular cell adhesion molecule-1 (sVCAM-1) ${ }^{(110)}$, tissue factor ${ }^{(119)}, \mathrm{MIF}^{(120)}$, MCP-1 ${ }^{(121)}$, soluble receptors of TNF (sTNFR) and for eotaxin, an inflammatory factor implicated in asthma, another complication of obesity ${ }^{(122)}$. Weight loss induced by gastric bypass reduced the circulating concentrations of visfatin ${ }^{(123)}$ and $\mathrm{TNF}-\alpha^{(124,125)}$. One study followed sixty obese patients during the course of weight loss induced by bariatric surgery and reported a reduction of $30 \%$ of initial weight, a decrease in CRP, SAA, orosomucoid protein, IL- 6 , TNF- $\alpha$ and fibrinogen concentrations, and an increase in adiponectin concentration ${ }^{(126)}$. After the surgery, the concentration of IL- 6 dropped slowly while the concentrations of SAA and CRP dropped more quickly ${ }^{(126)}$.

There was a significant modification in the expression of inflammatory genes in the subcutaneous adipose tissue of obese women following a hypoenergetic diet ${ }^{(127)}$. The expression of 100 genes linked to inflammatory processes was modified after 4 weeks ( $41 \%$ increased and 59\% decreased). These genes belonged to at least twelve functional families including cytokines, the complement factor cascade, acute-phase proteins of inflammation, and molecules involved in cellular adhesion and in the remodelling of the extracellular matrix. The improvement of the inflammatory profile (at the level of gene expression) involved both the decreased expression of pro-inflammatory factors and the increased expression of anti-inflammatory factors such as IL-10 and IL-1ra. The modification of the inflammatory gene expression profile was very similar in subjects following bariatric 

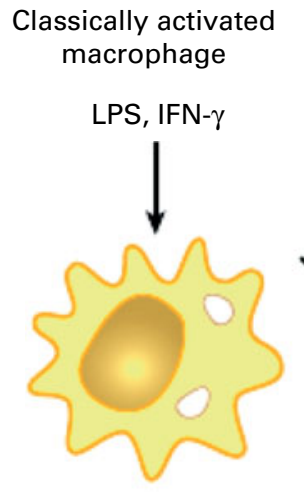

Alternatively activated macrophage

Fig. 3. Schematic representation of factors regulating macrophage polarity and insulin resistance in adipose tissue. Under lean conditions, adipocytes secrete factors, such as IL-13, that promote alternative activation of macrophages. Alternatively activated (M2) macrophages secrete anti-inflammatory mediators, such as IL-10, and may secrete insulin-sensitising factors. Obesity induces changes in adipocyte metabolism and gene expression, resulting in increased lipolysis and the release of pro-inflammatory NEFA and factors that recruit and activate macrophages, such as chemokines and TNF- $\alpha$. Activated M1 macrophages produce large amounts of pro-inflammatory mediators, such as TNF- $\alpha, \mathrm{IL}-1 \beta$ and resistin, that act on adipocytes to induce an insulin-resistant state. This establishes a positive feedback loop that further amplifies inflammation and insulin resistance. IFN, interferon; LPS, lipopolysaccharide. Reproduced with permission from Olefsky \& Glass ${ }^{(76)}$

surgery and was associated with a reduction of macrophage infiltration. In this study, the protein expression of IL-10 increased, suggesting a possible M1 to M2 (pro-inflammatory to anti-inflammatory) switch of macrophage phenotypes ${ }^{(31)}$. Overall, the mitigation of the systemic inflammatory profile observed during weight loss is associated with modifications of adipose tissue inflammatory gene expression, and this may be linked with altered profiles of inflammatory protein secretion. The eventual consequence of this phenomenon on the local biology of the adipose tissue remains to be identified.

\section{Chronic low-grade inflammation and insulin resistance \\ Experimental model systems in vitro}

In obesity and the metabolic syndrome, key organs displaying increased insulin resistance are the liver, skeletal muscle, adipose tissue and the endothelium (Fig. 6). Experimental model systems in vitro have shown that hepatocytes as well as muscle cells, adipocytes and endothelial cells respond to exposure to the pro-inflammatory cytokines TNF- $\alpha$, IL-6 and/or IL-1 $\beta$ with impaired insulin signalling ${ }^{(128)}$. Since these cytokines mediate their effects by binding to their cognate receptors, downstream components of the signalling cascade must interfere with insulin receptor function. Several such pathways have been identified (Fig. 7) ${ }^{(128)}$. Proinflammatory cytokine signalling usually activates the kinases
IкB kinase $\beta$ and $\mathrm{C}$-jun $\mathrm{N}$-terminal kinase 1 , both of which in turn phosphorylate the Ser 307 residue of insulin receptor substrate (IRS)-1. This prevents the phosphorylation of tyrosine residues of IRS-1 by the insulin receptor and the downstream signalling cascade. TNF- $\alpha$ mediates insulin resistance also via the activation of p38 mitogen-activated protein kinase (MAPK) which interferes with the IRS-phosphatidylinositol 3-kinase-Akt pathway ${ }^{(129)}$. Another mechanism involves cytokine-induced suppressor of cytokine signalling 1 and 3, which also prevent tyrosine phosphorylation of IRS proteins, by direct interference, or ubiquitylation and subsequent degradation ${ }^{(130,131)}$. Induction of suppressor of cytokine signalling 3 by IL-6 occurs via the signal transducer and activator of transcription 3 (STAT3) mammalian target of rapamycin (mTOR) pathway ${ }^{(132)}$.

\section{In vivo models}

The impairment of insulin signalling by TNF- $\alpha$ has also been observed in vivo after infusion of the cytokine into rodents $^{(133,134)}$. A critical mediator downstream of TNF- $\alpha$ appears to be MIF, since mice with a disrupted $M I F$ gene preserve normal insulin signalling ${ }^{(135)}$. In this context, it is of interest that adipocytes are able to secrete $\mathrm{MIF}^{(33)}$. The latter finding demonstrates that there are still substantial gaps in our understanding of the pro-inflammatory cytokine signalling 


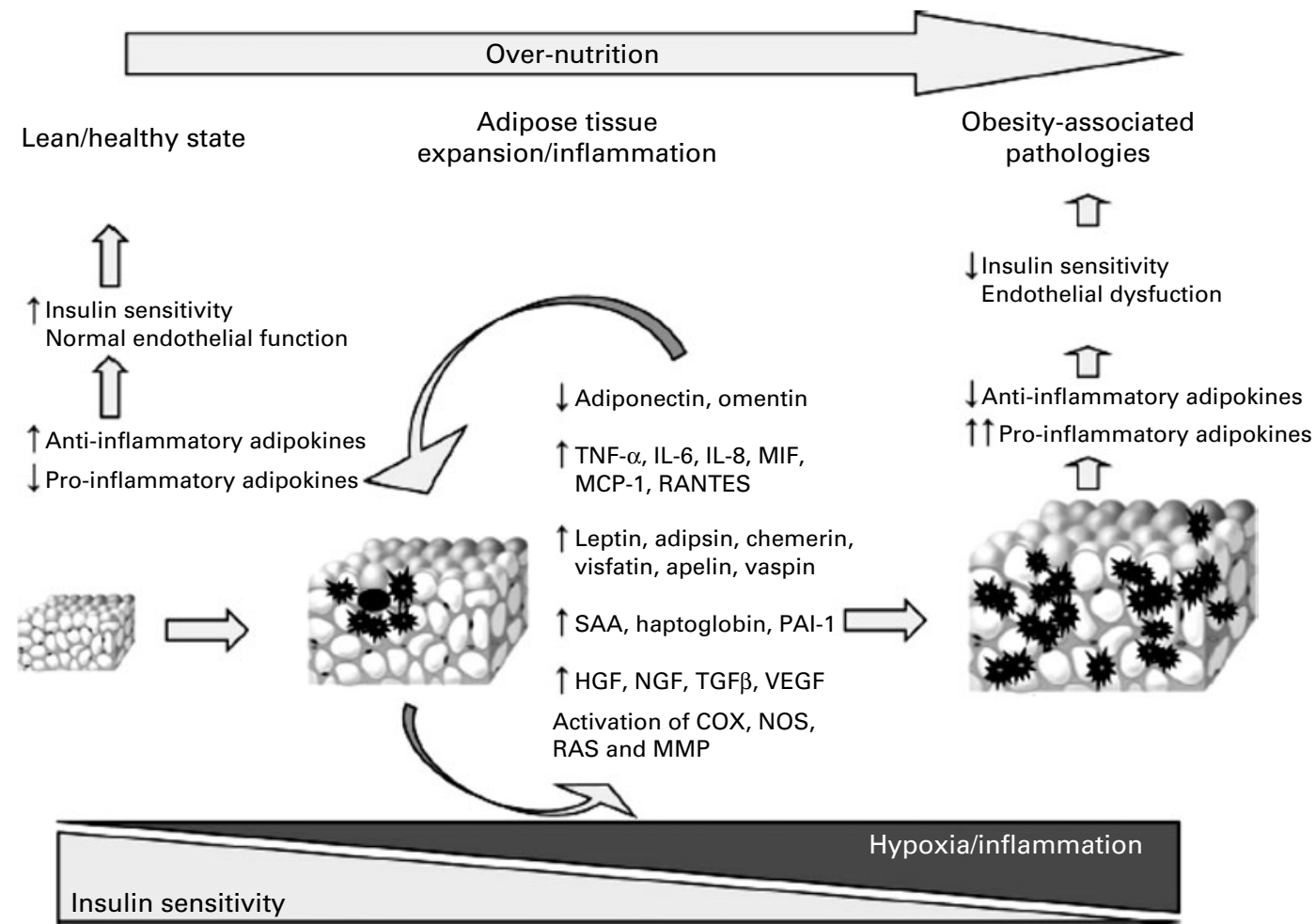

Fig. 4. Schematic representation of the alterations in adipose tissue that accompany body-weight gain. In the lean state, the tissue secretes elevated levels of adiponectin and other anti-inflammatory adipokines and is insulin responsive. Energy intake in excess of expenditure is followed by adipocyte hypertrophy and death and chemotactic adipokine release (see Fig. 1). This facilitates macrophage infiltration into the tissue and exacerbates the inflammatory response. These secretory changes are accompanied by local insulin resistance and hypoxia. Many of the adipokines released by inflamed adipose tissue cause insulin resistance and endothelial dysfunction. COX, cyclo-oxygenase; HGF, hepatocyte growth factor; MIF, macrophage migration inhibitory factor; MMP, matrix metalloproteinase; NGF, nerve growth factor; NOS, NO synthase; PAI-1, plasminogen activator inhibitor-1; RANTES, regulated on activation, normal T expressed and secreted; SAA, serum amyloid A; TGF, transforming growth factor; VEGF, vascular endothelial growth factor; MCP, monocyte chemoattractant protein; RAS, renin-angiotensin system. Reproduced with permission from Karastergiou \& Mohamed-Ali ${ }^{(77)}$.

cascade leading to insulin resistance. Most importantly, it remains unclear where other known regulators of insulin sensitivity fit into the chain of events. There is convincing evidence that reactive oxygen species (ROS) are critical to the effects of TNF- $\alpha$ on insulin signalling ${ }^{(136)}$, and also that mitochondrial dysfunction is involved ${ }^{(137)}$. The impact of insulin on cellular metabolic activity, proliferation and differentiation can also be impaired by inflammatory mediators via an indirect pathway, i.e. by enhancing or suppressing the production of hormones that modulate cellular responses to insulin. These effects include the up- or down-regulation of the synthesis of resistin ${ }^{(138)}$, leptin, $\operatorname{adiponectin}^{(139)}$, lipocalin $2^{(140)}$, osteopontin ${ }^{(141)}$, and of insulin itself. When considering the subnanomolar systemic concentration of many proinflammatory mediators (see section 'Obesity and low-grade inflammation'), it is possible that only a few of them contribute to the metabolic derangements seen in obesity and the metabolic syndrome.

A different situation emerges when paracrine effects of inflammatory mediators are considered. As described above, there is substantial local production of inflammatory mediators in organs affected by insulin resistance. Hepatocytes, adipocytes, muscle cells and the endothelium are sites of inflammatory mediator synthesis, but local activated macrophages appear to be the dominant site of synthesis and secretion, which leads to spillover into the general circulation ${ }^{(66,67,142)}$. Paracrine concentrations of inflammatory mediators are sufficient to induce insulin resistance ${ }^{(128)}$. Indeed, co-culture of adipocytes with macrophages caused impairment of insulin signalling ${ }^{(75)}$. In addition to paracrine effects, it is conceivable that the functions of liver cells are affected by inflammatory mediators released from the abdominal adipose tissue because of their blood link.

\section{Evidence supporting the link between inflammatory mediators and insulin resistance}

In human subjects, the most direct approach to assess the contribution of low-grade inflammation to the development of insulin resistance and the metabolic syndrome is to analyse the consequences of anti-inflammatory pharmacotherapy. The longest experience is with the use of salicylates which are weak inhibitors of IкB kinase $\beta$ and of serine phosphorylation of IRS proteins ${ }^{(143,144)}$. Early clinical trials with high doses of salicylates, notably aspirin, yielded both positive and negative effects on glycaemia and insulin resistance. Later studies have revealed that only very high doses are effective in improving glucose metabolism ${ }^{(145)}$. A randomised placebo-controlled pilot trial of salsalate treatment for 1 month in twenty non-diabetic obese individuals found decreased 


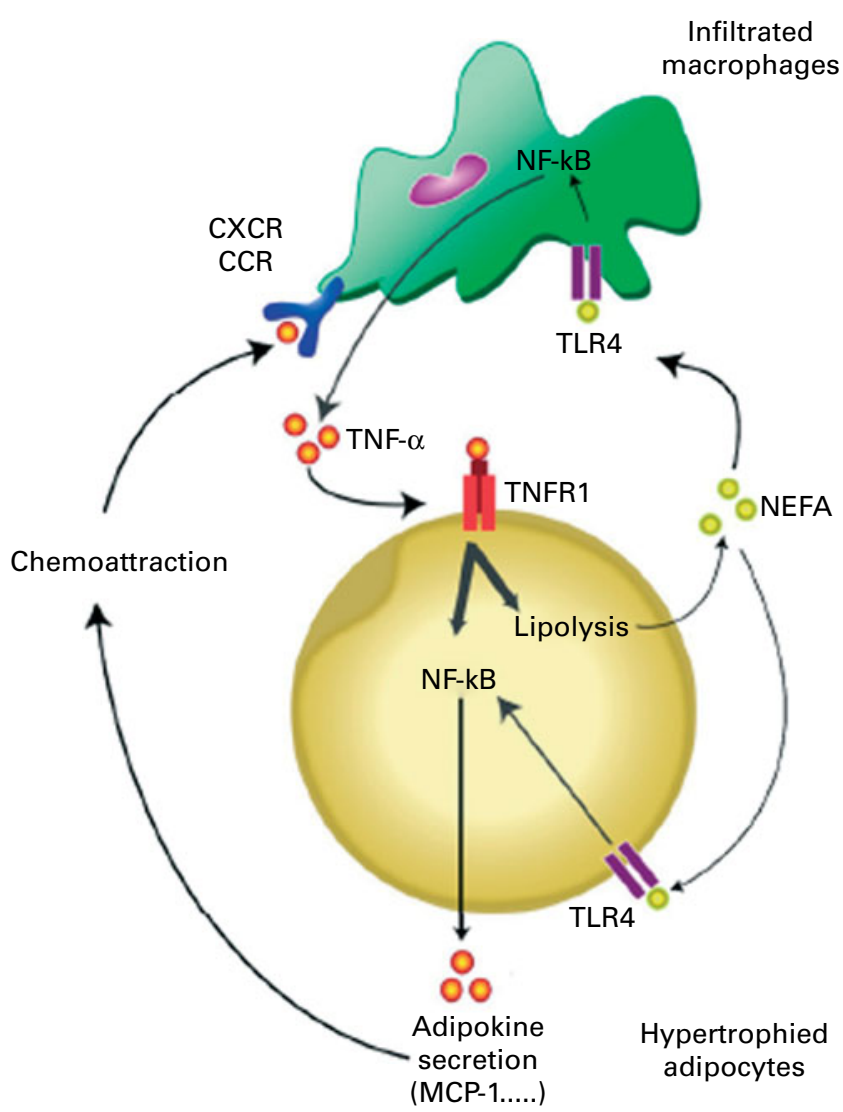

Fig. 5. Schematic representation of the cross-talk between adipocytes and macrophages of adipose tissue in obesity. TNF- $\alpha$ produced by macrophages activates adipocytes via TNF- $\alpha$-receptor-1 (TNFR1) and the NF-KB pathway. TNF- $\alpha$ also induces lipolysis leading to the release of NEFA. Saturated NEFA in turn activate the Toll-like receptor 4 (TLR4)/NF-kB pathway in both macrophages and adipocytes, thereby further amplifying the inflammatory process. Some of the adipokines produced (e.g. monocyte chemoattractant protein-1 (MCP-1)) exert chemoattractant activity through binding to specific receptors (CXC chemokine receptor (CXCR) and CC chemokine receptor $(\mathrm{CCR})$ ) of macrophages, leading to their infiltration in obese adipose tissue. Reproduced with permission from Maury \& Brichard ${ }^{(94)}$.

blood glucose and insulin responses to an oral glucose challenge consistent with improved insulin sensitivity ${ }^{(146)}$. A secondary analysis of a prospective multicentre observational study of 4905 adults with rheumatoid arthritis, of whom 1808 had taken hydroxychloroquine, indicated a reduced risk of diabetes in patients using this $\operatorname{drug}^{(147)}$. More specific anti-inflammatory intervention is possible through the use of recombinant proteins antagonising proinflammatory mediators. A first controlled double-blind trial was performed with daily injections of recombinant human IL-1ra for 13 weeks. This resulted in decreased glycated $\mathrm{Hb}$ levels and enhanced endogenous insulin production ${ }^{(148)}$. However, although there was a significant decrease in systemic CRP and IL-6 concentrations in response to anti-inflammatory treatment, there was no change in insulin resistance (homeostasis model assessment index and euglycaemichyperinsulinaemic clamp studies). It is difficult to judge the extent of inflammation persisting during therapy because absolute serum concentrations of CRP and IL-6 were not reported. There was no significant decrease in circulating
TNF- $\alpha$, MCP-1 or IL- 8 concentrations, which indicates that there was no general down-regulation of pro-inflammatory cytokines. Another target-specific approach is the neutralisation of TNF- $\alpha$ by injections of recombinant antibodies or sTNFR. In animal models of insulin resistance, infusion of TNF- $\alpha$ antibodies has been reported to ameliorate insulin signalling ${ }^{(8,149)}$. In obese non-diabetic or diabetic individuals, several studies have observed improvement of insulin sensitivity after prolonged treatment with neutralising TNF- $\alpha$ antibodies $^{(150,151)}$, whereas other trials did not report such effects of TNF- $\alpha$ antibody injections, despite dampening of systemic inflammation ${ }^{(152)}$. Possible explanations are that the recombinant antibodies do not reach sufficiently high concentrations in target tissues, or that TNF- $\alpha$ neutralisation is effective only in skeletal muscle tissue but not in adipose tissue as has been observed in rats ${ }^{(153)}$. The overall conclusion is that results of studies of anti-inflammatory therapy generally support the concept of inflammatory mediators as contributors to the pathogenesis of insulin resistance, but have as yet not provided clear evidence of a critical pathogenic role of TNF- $\alpha$ or IL-1.

\section{Postprandial inflammatory response}

The foregoing discussion has dealt with chronic changes in concentrations of inflammatory mediators but a rise in inflammation also appears to take place acutely following meals. The postprandial inflammatory response lasts for only few (4-8) h but it recurs several times a day following eating. Although the postprandial inflammatory response has been known for several years ${ }^{(154)}$, it is only recently that its probable importance in the generation of insulin resistance and atherosclerosis has been appreciated ${ }^{(155-157)}$. Several cells in the body associated with the innate immune system, including abdominal adipocytes and monocytes/ macrophages, respond to acute postprandial elevation of several components of a meal by mounting a transient inflammatory response. The most efficient triggers of the postprandial inflammatory response appear to be TAG, SFA, oxysterols and glucose ${ }^{(158-161)}$. The pathophysiological significance of a postprandial inflammatory response in causing insulin resistance, the metabolic syndrome and atherosclerosis is currently under investigation, and this response appears to play a much more crucial role than previously thought ${ }^{(162)}$.

\section{Non-dietary factors influencing the magnitude of the postprandial inflammatory response}

Body weight. Obesity is considered an important determinant of the magnitude of the postprandial inflammatory response ${ }^{(163)}$, perhaps being more important than any specific component of a meal inducing the response. The exaggerated postprandial inflammatory response of the obese is reversible upon reduction of body weight ${ }^{(155,164)}$.

Hyperglycaemia and type 2 diabetes. Patients with type 2 diabetes exhibit a higher postprandial inflammatory response than non-diabetics, irrespective of their body weight ${ }^{(165,166)}$ 
Fig. 6. Schematic representation of the role of adipose tissue inflammation in the initiation and maintenance of systemic insulin resistance. Reproduced with permission from de Luca \& Olefsky ${ }^{(106)}$.

The magnitude of the postprandial inflammatory response appears to correlate with the degree of insulin resistance ${ }^{(165)}$.

Drugs. Certain medications including statins and angiotensin II receptor antagonists ameliorate the postprandial inflammatory response in obese patients ${ }^{(167)}$.

\section{Pathophysiology of the postprandial inflammatory response}

The daily influx of TAG, SFA, glucose and other food components initiates an acute innate immune (i.e. inflammatory) response that lasts for a few hours. Meals or food components may contain LPS which directly triggers systemic inflammation. Related to this effect, the absorptive process may allow translocation of LPS from gut bacteria into the systemic circulation $^{(168)}$. Meals may contain oxidised components which initiate oxidative stress and/or inflammatory responses upon absorption. Postprandial hyperglycaemia can suppress antioxidant capacity ${ }^{(169)}$ and thus its ability to curb an inflammatory reaction. Hyperglycaemia induces the production of free radicals which themselves initiate an inflammatory reaction. A six-transmembrane protein of prostate 2 (STAMP2) has been proposed as a major determinant of the postprandial inflammatory response ${ }^{(170)}$, acting to block activated inflammatory signalling pathways in adipocytes and possibly in macrophages. In vivo, feeding induces STAMP2 expression in visceral white adipose tissue ${ }^{(170)}$. Furthermore, the visceral tissue of STAMP gene knockout mice is resistant to insulin action ${ }^{(170,171)}$.

\section{Ageing and low-grade inflammation}

Ageing is associated with complex changes in, and a dysregulation of, the immune system, including its inflammatory component. The ageing of the immune system, immunosenescence, has been suggested to be a consequence of continuous attrition caused by chronic antigenic overload ${ }^{(172)}$. Ageing is accompanied by a low-grade, chronic inflammatory state clearly shown by 2- to 4-fold increases in serum levels of several inflammatory mediators in older persons ${ }^{(173)}$. Studies have reported increased plasma/serum levels of the proinflammatory cytokine IL- 6 in healthy subjects with advanced age $(55-75 v .26-54 \text { years })^{(174)}$, an increase of $0.016 \mathrm{pg} / \mathrm{ml}$ per year of life $\mathrm{e}^{(175)}$ and a significant increase with overall age (from 20 to 102 years) ${ }^{(176)}$, and in elderly diabetic subjects $(65-80 \text { years })^{(177)}$. Ageing is also associated with increased concentrations of TNF- $\alpha^{(178,179)}, \mathrm{CRP}^{(180)}$ and IL-1ra ${ }^{(176,181)}$. It is hypothesised that failure of anti-inflammatory mechanisms to neutralise inflammatory processes that are continuously triggered lifelong plays a role in chronic low-grade inflammation in the elderly ${ }^{(182)}$. In line with this, it has recently been shown that ageing (two groups with a mean age of 77.9 and 102.5 years, respectively $v$. a group with a mean age of 43.5 years) is characterised by a profound reduction in anti-inflammatory lipoxin $\mathrm{A}_{4}$ levels ${ }^{(183)}$.

The effect of ageing on the immune system, however, cannot be completely separated from the contribution of co-morbidity, medication use or malnutrition ${ }^{(184,185)}$. Since several inflammatory markers act as disease markers, it is possible that some of the chronic low-grade inflammation patterns found in the elderly may be related to the presence of co-morbidities ${ }^{(180,186)}$. Interestingly, however, successful ageing (ageing without co-morbidities) has also been associated with chronic low-grade inflammation ${ }^{(173)}$. Other factors that may affect and modulate circulating levels of inflammatory mediators, including obesity, infections, physical activity, age-related decline in sex hormones and altered host-microbiota interaction at the gut level, may also be involved in the age-associated increase in lowgrade inflammation ${ }^{(172,187-189)}$. Furthermore, high plasma 


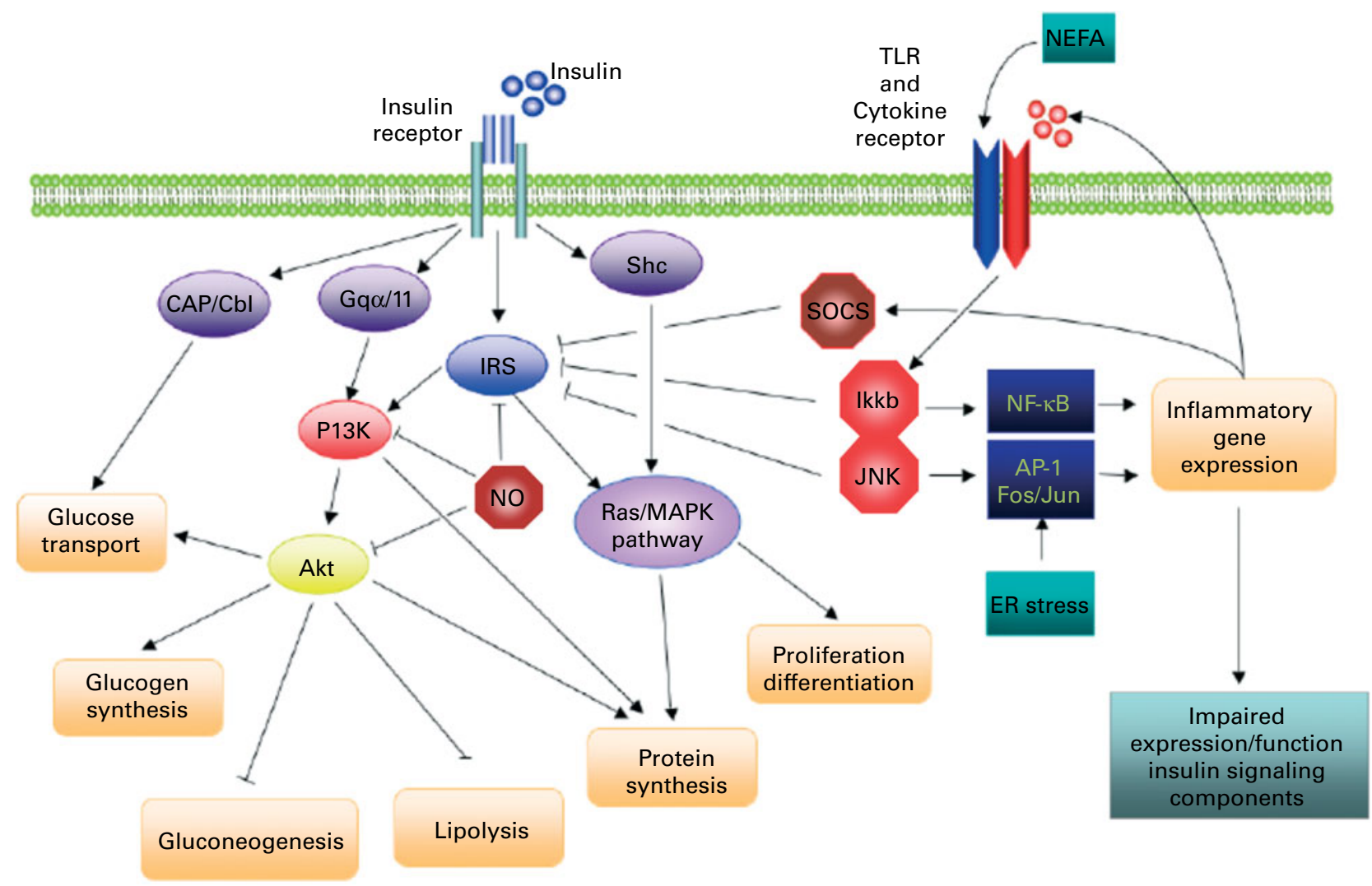

Fig. 7. Schematic representation of the direct interaction between inflammatory and insulin signalling pathways. The insulin signalling cascade branches into two main pathways. The PI3K-Akt pathway mediates insulin action on nutrient metabolism including glucose uptake. The Ras-mitogen-activated protein kinase (MAPK) pathway mediates the insulin's effect on gene expression, but also interacts with the PI3K-Akt pathway to control cell growth and differentiation. Activation of the insulin receptor leads to tyrosine phosphorylation of insulin receptor substrate (IRS)1, thereby initiating signal transduction. Stimulation of the $\mathrm{NF}-\kappa \mathrm{B}$ and activator protein-1 (AP-1) Fos/Jun inflammatory pathways results in the activation of serine kinases, Ikk $\beta$ and C-jun N-terminal kinase 1, which reduce the signalling ability of IRS1. Related negative regulators of IRS proteins include the suppressor of cytokine signalling proteins and NO, which are induced in inflammation, and promote IRS degradation. NO also reduces PI3K-Akt activity by nitrosylation of Akt. Reproduced with permission from de Luca \& Olefsky ${ }^{(128)}$. TLR, Toll-like receptors.

levels of IL-6 (and TNF- $\alpha$ ) in the elderly were associated with truncal fat mass ${ }^{(177)}$, suggesting that some of this effect might be mediated with age-associated increase in fat mass.

There is strong evidence that low-grade elevations of circulating inflammatory mediators are associated with the development of age-related conditions such as atherosclerosis, cognitive decline and frailty. This may in part reflect the inflammatory nature of these conditions which involve local or generalised inflammation (e.g. neuroinflammation in cognitive decline), with the increase in circulating concentrations of inflammatory mediators reflecting overspill from the inflammatory lesion(s). Additionally, the increased inflammatory burden could make a contribution to the ongoing pathology and to a worsening clinical situation. Increases in the levels of circulating TNF- $\alpha, \mathrm{IL}-6, \mathrm{IL}-2 \mathrm{R}$ and CRP are also strong predictors of all-cause mortality risk in longitudinal studies of several elderly cohorts ${ }^{(190-193)}$. However, whether increased inflammatory activity causes age-associated pathology or reflects the sum of ongoing pathological processes ${ }^{(173,194)}$ remains uncertain. Survival analyses in studies from the USA and Europe with several populations (healthy, non-disabled, $\geq 65$-year-old subjects $^{(191)}$, high-functioning subjects aged $70-79$ years ${ }^{(192)}$, disabled women aged $\geq 65$ years $^{(193)}$ and relatively healthy 80-year-old people $\left.{ }^{(190)}\right)$, however, show that effects of inflammatory mediators were independent of pre-existing morbidity and other traditional risk factors for death. This indicates that these inflammatory mediators influence pathological processes or act as very sensitive markers of subclinical disorders in the elderly ${ }^{(173)}$.

\section{Exercise and low-grade inflammation}

Influence of acute and regular physical activity and fitness on low-grade inflammation

The health benefits of a physically active lifestyle are well recognised. Physical inactivity and obesity are also increasingly recognised as modifiable behavioural risk factors for a wide range of chronic diseases, and in particular for $\mathrm{CVD}^{(195)}$ Physical fitness, physical exercise and physical activity are often used as interchangeable concepts, but it is important to point out the differences among these. Physical activity is any body movement that increases energy expenditure ${ }^{(196)}$. 
Self-reported data of physical activity are easy and feasible to ask in a questionnaire or interview in large populations but are a measurement subject to recall and reporting biases. Exercise is planned, structured and repetitive physical activity, while physical fitness is the capacity to perform physical activity, and makes reference to a full range of physiological and psychological qualities. To eliminate reporting bias that could be present in self-reported physical activity measurement, several studies have examined the relationship between cardiorespiratory fitness and inflammatory markers. Maximal oxygen consumption $\left(\mathrm{VO}_{2 \max }\right)$ attained during a graded maximal exercise to voluntary exhaustion is considered as the single best indicator of cardiorespiratory fitness ${ }^{(197)}$. There are excellent reviews of the evidence addressing the influence of physical activity and fitness on low-grade inflammation from epidemiological studies as well as clinical trials on the general adult population ${ }^{(198-202)}$, athletes ${ }^{(203,204)}$, and in children and adolescents ${ }^{(205)}$.

Acute $v$. regular exercise. IL-6 and other cytokines that are produced and released by skeletal muscles have been suggested to be involved in mediating the health-beneficial effects of exercise and to play important roles in the protection against diseases associated with low-grade inflammation. The following chain of events is based on observations by Pedersen and colleagues and has been excellently reviewed elsewhere $^{(206-208)}$ :

(1) Contracting skeletal muscle is a major source of circulating IL-6 in response to acute exercise. Plasma IL-6 increases in an exponential fashion with exercise and is related to exercise intensity, duration, the mass of muscle recruited and endurance capacity. During heavy exercise, such as a marathon, there is up to a 60 -fold increase in plasma IL-6 concentration ${ }^{(209)}$, with the duration of the event explaining more than $50 \%$ of the variation in concentration ${ }^{(210)}$. Interestingly, IL-6 shows a markedly lower response to acute exercise in trained subjects.

(2) Physiological concentrations of IL-6 stimulate the appearance in the circulation of the anti-inflammatory cytokines IL-1ra and IL-10 and inhibit the production of the proinflammatory cytokine TNF- $\alpha$. The health benefits of long-term regular exercise are ascribed to the anti-inflammatory response elicited by an acute bout of exercise, which is partly mediated by muscle-derived IL-6.

(3) The anti-inflammatory effects of exercise may therefore offer protection against TNF-induced insulin resistance. Moreover, IL-6 stimulates lipolysis as well as fat oxidation. The increase in IL- 6 at the end of exercise is responsible for the increased CRP levels during late recovery.

In response to regular physical activity, basal as well as post-exercise plasma concentrations of IL-6 will decrease by mechanisms that might include increased glycogen content, improved antioxidant capacity and improved insulin sensitivity. The lower concentrations of IL- 6 in the circulation will subsequently result in lower CRP levels.

Few studies have prospectively examined the effect of exercise training on low-grade inflammatory status, and the data obtained from intervention studies are less consistent when compared with cross-sectional population studies. A lower number of subjects or a good physical condition in the start of some intervention studies may explain a part of this inconsistency. Nevertheless, two longitudinal studies in athletes show that regular training induces a reduction in CRP concentration ${ }^{(211,212)}$. Conflicting findings exist in clinical trials that have involved exercise only. Several training interventions have not produced changes in basal IL-6 or CRP concentrations ${ }^{(213-218)}$, while significant reductions in inflammatory markers have been observed following training without changes in BMI or body fat in elderly participants ${ }^{(219,220)}$. The largest trial was performed in 652 sedentary healthy, young and middle-aged, white and black women and men in the HEalth, RIsk factors, exercise Training And Genetics (HERITAGE) Family Study ${ }^{(221)}$. They were subjected to a 20-week standardised exercise training programme; there was no control group, and each subject served as its own control. A non-significant reduction in CRP concentration was consistent across all groups and varied between 1.2 and $2 \cdot 2 \mathrm{mg} / \mathrm{l}$. Considering that the over-time variation in CRP in healthy individuals with stable lifestyle is small ${ }^{(22)}$, the reduction, although not significant, could nevertheless reflect the true effect of exercise training. Further stratification according to basal CRP levels showed a reduction by about $1.3 \mathrm{mg} / \mathrm{l}$ in subjects with initial CRP levels above $3.0 \mathrm{mg} / \mathrm{l}$.

Effects of exercise in elderly people. Elderly people have higher basal levels of inflammation independently of disease status, and a considerable number of studies have been carried out in this population to assess associations between physical activity and inflammatory markers ${ }^{(190,223-230)}$. Rather consistent inverse, BMI-independent, associations are found and the associations are suggested to be dosedependent; the more physically active the person, the lower the inflammatory markers ${ }^{(208,224)}$. Also subjects over 80 years of age show consistent inverse associations between inflammation and physical activity ${ }^{(190)}$. Functional fitness was inversely associated with IL- 6 and IL-1ra concentrations (but not with CRP, TNF- $\alpha$, IL-10 or IL-1 $\beta$ ) in a prospective population-based study of 1020 participants aged 65 years and older $^{(223,231)}$. Muscle strength was also evaluated in this study and low hand-grip strength was associated with high levels of CRP and IL- $6^{(231)}$. Other studies have also shown a negative association of CRP, IL- 6 and TNF- $\alpha$ with muscle strength ${ }^{(228,232)}$.

Exercise intervention in elderly people or in patients with CVD shows consistent anti-inflammatory effects. After a 6-month individualised, supervised exercise programme for forty-three subjects at high risk of IHD, a $35 \%$, albeit non-significant, reduction in CRP concentration was observed. The subjects exercised for a mean of 2.5 (range $0 \cdot 3-7 \cdot 4) \mathrm{h} /$ week $^{(233)}$. One reason for the lack of a significant effect despite the fairly large reduction in CRP concentration is the small size of the study. Another study reported a decrease in basal plasma IL-6 concentration after aerobic training in patients with coronary artery disease ${ }^{(234)}$. A randomised trial of thirty-nine patients with intermittent claudication demonstrated that both serum CRP and SAA concentrations were significantly reduced after 3 and 6 months of supervised 
exercise compared with controls ${ }^{(235)}$. In a relatively large intervention study of exercise training on cardiac rehabilitation patients, the median CRP concentration was reduced by $41 \%$, while CRP concentrations did not change in subjects who did not exercise ${ }^{(236)}$. Again, the exercise training seemed to be more effective in those with the highest initial CRP concentrations, independently of changes in body weight or percentage of body fat, indicating that baseline levels of low-grade inflammation may be an important factor.

Studies in patients with diabetes ${ }^{(237)}$ or the metabolic syndrome $e^{(238,239)}$ have consistently demonstrated inverse associations between fitness and inflammation, independently of fatness. In one study, the independent associations of fitness were in fact more prominent among metabolic syndrome patients compared with healthy participants ${ }^{(238,239)}$.

Effects of exercise in middle-aged and younger adults and in children. Several studies of large population cohorts, such as the British Regional Heart Study ${ }^{(225)}$, the Greek ATTICA study ${ }^{(240)}$, the Third National Health and Nutrition Examination Survey (NHANES) ${ }^{(241,242)}$, the Men's Health Professionals' Follow-Up Study ${ }^{(243)}$, the Nurses' Health Study ${ }^{(243)}$ and the Women's Health Study ${ }^{(244)}$, provide evidence for an inverse, independent dose-response relationship between plasma CRP concentration and level of physical activity in both men and women, but the consistency is less than in elderly subjects, or in disease states. In contrast, the associations found between self-reported physical activity and TNFR1, TNFR2, IL-6 and CRP concentrations in a study including healthy men from the Men's Health Professionals' FollowUp Study and healthy women from the Nurses' Health Study ${ }^{(243)}$ were no longer significant when adjusting for BMI and leptin. Thus, the effect of physical activity on circulating markers of low-grade inflammation appears to be mediated by weight loss. In another study in healthy men and women, BMI, but not previous year or current physical activity, predicted CRP concentration ${ }^{(245)}$. Similarly, a crosssectional study ${ }^{(246)}$ in men found no relationship between leisure-time physical activity and CRP, fibrinogen and SAA concentrations, after correction for BMI and current smoking status. CRP levels in 2120 Finns were associated with obesity indices and physical activity among both sexes ${ }^{(247)}$; in multivariate analyses, the determinants of CRP concentration included obesity and smoking in men, and obesity, oral contraceptive use and physical activity in women. The study showed that about one in three of healthy women who used oral contraceptives had a CRP concentration exceeding $3 \mathrm{mg} / \mathrm{l}$, which should be taken into account when studying younger females. Cross-sectional studies in men from the Aerobics Center Longitudinal Study have demonstrated that cardiorespiratory fitness levels are inversely associated with CRP concentration and also the prevalence of elevated CRP concentrations ${ }^{(248)}$. Analyses with fibrinogen and white blood cell count showed similar results ${ }^{(249)}$. The competing effect of weight and fitness (assessed by submaximal graded exercise treadmill testing) on cardiorespiratory fitness levels was studied in the NHANES, which included 2112 US adults without previously diagnosed $\mathrm{CVD}^{(250)}$. Both fitness and BMI were independently associated with increased fasting insulin and CRP concentrations. However, when patients with low, moderate and high fitness were further stratified as normal, overweight or obese, weight remained significantly associated with CRP, but fitness did not. This study concludes that 'fat but fit' subjects require weight-loss interventions to improve their CRP levels. Future interventions should emphasise weight control, even for those with high cardiorespiratory fitness.

In disease-free young populations, studies have assessed the interaction between inflammatory markers (CRP, IL-6 and TNF- $\alpha$ ), physical activity or cardiorespiratory fitness and fatness ${ }^{(47,251-258)}$. Organised leisure-time exercise (assessed by a questionnaire) in children has shown negative correlations with serum IL-6 concentrations, independently of adiposity and fat localisation ${ }^{(256)}$, and in 10-year-old children, a borderline significant negative association was observed between CRP and self-reported physical activity, independently of ponderal index ${ }^{(47)}$. US children and young adults (aged 6-24 years) from the Columbia University BioMarkers Study showed an inverse correlation between cardiovascular fitness and CRP concentration but only in boys, which remained after adjustment of confounders including $\mathrm{BMI}^{(251)}$. Only one study has used accelerometry (an objective measure of total physical activity compared with leisure-time physical activity or exercise) instead of questionnaires as well as cardiovascular fitness ${ }^{(257)}$. In this study of 9-year-old Swedish children, total physical activity was unrelated to CRP, fibrinogen, C3 or C4 concentrations, but exercise was. Nevertheless, once body fat was entered into the regression models, no associations with cardiovascular fitness or physical activity and the inflammatory markers measured were observed ${ }^{(257)}$. Similarly, no associations were found between cardiorespiratory fitness or self-reported physical activity and CRP concentration in 12-year-old healthy Welsh children ${ }^{(259)}$

CRP, C3 and ceruloplasmin (but not C4) concentrations were negatively associated with muscle strength after controlling for sex, age, pubertal status, weight, height, socio-economic status and cardiorespiratory fitness, but did not remain when adjusting for body fat. Nevertheless, when stratifying according to overweight status, CRP (but not C3, C4 or ceruloplasmin) concentration was associated with muscle strength in overweight, but not in normal-weight, adolescents after controlling for potential confounders, including body fat and fat-free mass ${ }^{(260)}$.

\section{Conclusions for effects of physical activity and fitness on low-grade inflammation}

Most research on this topic hypothesised that the association between fitness and inflammatory factors is independent of fatness. Given that physical activity and obesity are often inversely related, it is not clear as to whether the antiinflammatory health benefits of a physically active lifestyle are due to exercise per se or result from favourable changes in body composition. Related anti-inflammatory effects could be mediated by increased insulin sensitivity and/or improved concentrations of HDL-cholesterol, ROS or endothelial function, which all demonstrate anti-inflammatory actions ${ }^{(261)}$, and are related to both body fat and exercise. A systematic 
review addressed whether fitness or fatness has the greatest impact on inflammatory factors ${ }^{(198)}$. The review concluded that both fitness and fatness are associated with systemic inflammatory status, although the relative contributions of both may be dependent on age, disease status and sex. These determinants do most probably involve a strong background of low-grade inflammatory status, which consistently is shown to determine any possible inverse association. Although increasing physical activity may be an effective therapy for weight loss and may also emerge as a promising treatment for reducing overall inflammation, the magnitude of the effect to produce clinically meaningful results in the general population requires further research ${ }^{(200)}$. Nevertheless, exercise is uniquely positioned to reduce inflammation, and even small non-significant reductions in CRP levels may contribute to clinical benefits by reducing cardiovascular and metabolic risk ${ }^{(222)}$.

\section{A consideration of different approaches to identify relationships between diet and its components and markers of chronic low-grade inflammation}

The following sections review the effects of dietary factors, including dietary patterns, whole foods, individual nutrients and other bioactive components on the markers of lowgrade inflammation described above. Due to the physiological complexities detailed above, assessment of effects requires careful attention to treatment interventions and study designs, in the context of the endpoints described. Here, important aspects of study design are briefly mentioned.

\section{Epidemiological studies}

Epidemiological studies, where available, are discussed for each of the dietary factors. As mentioned above, bodyweight changes and exercise may have profound effects on biomarkers of low-grade inflammation; therefore, adjustments for BMI and activity level are critical. An accurate assessment of the analysed dietary parameter is also necessary. Although difficult to rectify, it is important to consider cultural differences in food consumption habits, for example differences in coffee preparation procedure and brew strength between the USA and Europe. Attention should also be paid to the assessment of dietary patterns. For example, the Mediterranean diet has been variously defined and scored as inclusive or exclusive of fish, poultry, dairy, eggs, moderate alcohol consumption and ratio of monounsaturated:saturated fat. However, it is not clear whether these differences in scoring would result in alternative conclusions being drawn, and as such, this body of literature is best viewed in totality.

\section{Intervention studies}

Intervention studies, where available, are also discussed for each of the dietary factors. The studies presented here typically fall into three design categories: (1) chronic dietary interventions in individuals with some degree of existing low-grade inflammation, based on changes in markers measured in fasting blood, sometimes in the context of weight loss; (2) acute dietary interventions in which the acute effect of a putative anti-inflammatory dietary treatment is assessed against some background level of low-grade inflammation; (3) challenge studies in which inflammation is induced by either a dietary or exercise challenge, in the presence or absence of a putative anti-inflammatory dietary treatment. As with epidemiological studies, body weight and activity levels require careful control and monitoring because of their possible impact on low-grade inflammation. Chronic interventions are most commonly either parallel-arm designs or cross-over designs, and may be most relevant since they directly evaluate the effect of the dietary component on low-grade inflammation. However, acute intervention and postprandial challenge studies may also provide valuable insight, and postprandial inflammation has recently been hypothesised to play an aetiological role in the progression of CVD. Additionally, between-subject variations in biomarkers of the inflammatory response can be controlled for statistically in cross-over designs. In contrast to epidemiological studies, which often have a large sample size and may evaluate outcomes over a long duration, intervention studies are most often relatively small and of short duration (hours for acute studies; weeks to months for chronic studies). Small studies can limit power to identify significant effects. Another difficulty with intervention studies can be compliance among subjects; although an ideal study design would include actions to ensure compliance and would monitor this, such approaches are not always considered. Lack of compliance may limit the effectiveness of an intervention.

\section{Dietary patterns and low-grade inflammation}

For the purposes of this article, low-grade inflammation was defined as elevated circulating concentrations of proinflammatory cytokines, acute-phase proteins and adhesion molecules, and low circulating concentrations of adiponectin.

\section{Eating patterns}

Studies on diet and disease have traditionally examined the associations of individual nutrients, foods or food groups with risk factors and health outcomes. However, this approach has certain limitations: many nutrients are highly correlated, and have synergistic or interactive effects; examination of nutrients or foods singly may not provide enough statistical power due to the small effect size; and the possibility of finding significant associations by chance alone, due to multiple testing, is large. In response to the challenges of the traditional approach to understanding diet-disease relationships, more recently, nutrition epidemiologists have studied dietary or eating patterns that examine combinations of foods and nutrients, in relation to health and disease ${ }^{(262,263)}$. Dietary pattern research is generally based on two kinds of methods: a priori using diet scores; or a posteriori using data-driven techniques such as factor analysis and cluster analysis ${ }^{(264-266)}$.

Hypoenergetic diet. One diet-dependent but apparently quite non-specific way of decreasing low-grade inflammation 
is energy restriction ${ }^{(267)}$. Weight loss is accompanied by decreased concentrations of circulating mediators of inflammation, such as CRP, TNF- $\alpha$, IL-6 and sICAM-1 ${ }^{(155,268-270)}$, although it is difficult to dissect whether this effect is due to the weight loss per se or to the nature of the diet used to induce weight loss. On the other hand, it seems likely that reduced secretion of pro-inflammatory mediators from adipocytes or activated macrophages of adipose tissue contributes to the effect of weight loss ${ }^{(31,36,65,67,142)}$. However, energy restriction itself may also play an anti-inflammatory role, with key mediators of the effect being proteins of the sirtuin and Forkhead box, sub-group $\mathrm{O}$ (FoxO) families which are induced/activated during states of limited energy supply. The sirtuins are NAD + -dependent deacetylases of substrates ranging from histones to transcriptional regulators. As a consequence, metabolic efficiency is improved, cell defences against stress are strengthened and inflammatory activities are dampened, notably by decreasing the activation of $\mathrm{NF}-\mathrm{\kappa B}^{(271-274)}$. FoxO proteins are transcription factors which regulate the expression of genes involved in energy homeostasis, cell survival and inflammatory responses including $\mathrm{NF}-\kappa \mathrm{B}^{(274-278)}$. Early studies have shown that reduced energy intake is paramount compared with the nature of low-energy food, i.e. decreased concentrations of inflammatory markers are observed with a low-energy, fat-rich as well as with a low-energy, carbohydrate-rich diet ${ }^{(279)}$. However, it is conceivable that some dietary components are better regulators of sirtuin or FoxO activity than others. Resveratrol, present in red wine, was found to directly or indirectly interact with sirtuins and promote their deacetylase activity. This led to increased lifespan in model organisms and in animal models ${ }^{(280,281)}$. It is likely that dietary components will be identified that mimic or counteract the anti-inflammatory effects of energy restriction.

Mediterranean diet. The term Mediterranean diet refers to a traditional dietary pattern characteristic of many parts of Greece, Southern Italy, Southern Spain and elsewhere in the Mediterranean region. The traditional Mediterranean diet is rich in fruit, vegetables, whole-grains, legumes (beans), nuts, fish and low-fat dairy products, with moderate consumption of wine, and whose principal source of fat is olive $\mathrm{oil}^{(282-286)}$. Most studies have assessed the adherence to the Mediterranean diet by assigning a score in relation to the consumption of these foods. Others ${ }^{(284,285)}$ have used modified versions of this score by not considering certain food groups, i.e. dairy products.

Observational studies have examined the association of the Mediterranean diet with inflammatory markers in healthy persons ${ }^{(282,283,285)}$, and they generally report inverse correlations. In a recent study ${ }^{(282)}$ investigating psychological, behavioural and biological risk factors for subclinical CVD in 345 middleaged male twins, an inverse association between adherence to the Mediterranean diet and inflammation, as measured by plasma IL-6 concentration, was noted. This association was independent of several known cardiovascular risk factors, and persisted when twins within pairs were compared, suggesting that the results were not confounded by shared environmental and genetic factors. Although a marginal relationship between CRP concentration and the Mediterranean diet was observed, this association was no longer significant when other cardiovascular risk factors were considered in the models. It is likely that IL- 6 is a more sensitive marker of chronic low-grade inflammation and that CRP reflects a more downstream effect associated with IL-6. In a subsample of the Nurses' Health Study ${ }^{(285)}$, a Mediterranean diet index score was inversely associated with markers of inflammation (circulating IL-6 and CRP) as well as markers of endothelial dysfunction (the adhesion molecules sICAM-1, sVCAM-1 and soluble E-selectin (sE-selectin)); these associations persisted upon adjustment for traditional CVD risk factors. Similar findings were reported in the ATTICA study, involving 1514 men and 1528 women; specifically, subjects with greater adherence to the Mediterranean diet (those in the highest tertile) had $17 \%$ lower IL-6 and 20\% lower CRP concentrations, compared with those in the lowest tertile in analyses that adjusted for other cardiovascular risk factors ${ }^{(283)}$. Although a marginal association was noted between TNF- $\alpha$ and the Mediterranean diet, it was not significant in adjusted models ${ }^{(283)}$. In another observational study with obese subjects (625 men and 712 women with abdominal adiposity), those with high CRP levels $(>3 \mathrm{mg} / \mathrm{l})$ were less likely to adopt the Mediterranean $\operatorname{diet}^{(287)}$. The authors reported that adoption of the Mediterranean diet in conjunction with moderate physical activity was associated with a reduced likelihood of having high CRP levels by $72 \%$, highlighting the potential importance of the Mediterranean diet in diminishing inflammation. In subjects at high risk for CVD, those with diabetes or with multiple CHD risk factors, however, no association between the Mediterranean diet and CRP or adhesion molecule (sICAM-1 and sVCAM-1) concentrations was seen ${ }^{(288)}$. However, a significant relationship between higher consumption of some typical Mediterranean diet components (cereals, fruit, nuts and virgin olive oil) and circulating inflammatory markers (IL-6) and markers of endothelial function was noted in these at-risk subjects.

Few intervention studies have been conducted to examine the effect of consuming the Mediterranean diet on markers of low-grade inflammation. In two cross-over studies involving short-term interventions (1-3 months) with healthy subjects, differential effects of the Mediterranean diet on markers of inflammation (and endothelial dysfunction) were observed. In the study by Ambring et al. ${ }^{(289)}$, healthy subjects received their typical Swedish diet for about 4 weeks, and a Mediterranean-inspired diet for about 4 weeks in a randomised cross-over design, with 4 weeks of washout in-between. A marked reduction in the number of leucocytes, including monocytes, neutrophils and lymphocytes, and in the number of platelets after 4 weeks of the Mediterranean diet was noted, suggesting a lower inflammatory activity than during the Swedish diet period. On the other hand, IL-6 and CRP concentrations did not change with the Mediterranean diet; the authors speculated that the study may not have been powered to detect those effects. In another study ${ }^{(290)}$, twenty healthy young males were provided three dietary interventions, each lasting 4 weeks. First, all subjects consumed a diet high in saturated fat, next they were randomly assigned to either 
of the two intervention diets: a Mediterranean-style diet (MUFA-enriched diet, 22\% energy from MUFA) or a low-fat, high-carbohydrate diet $(<30 \%$ energy from fat and $55 \%$ from carbohydrates). LDL was isolated and oxidised. Oxidised LDL from subjects on either the Mediterranean diet or the high-carbohydrate diet decreased TNF- $\alpha$-induced VCAM-1 and E-selectin expression in human umbilical endothelial cells in vitro. A consistent decline in inflammatory markers has been observed in intervention studies involving obese subjects or those with the metabolic syndrome. In a randomised controlled study with 120 pre-menopausal obese women, the effects of a multidisciplinary approach (aiming at $10 \%$ weight reduction with a combination of a low-energy, Mediterranean-style diet and increased physical activity) were evaluated compared with a control group (given general information about healthy choices and exercise) ${ }^{(111)}$. The intervention group received regular sessions (18 over a 2-year period) with a nutritionist to ensure compliance. Significant reduction in several markers of inflammation (CRP, IL-6 and IL-18) and an increase in adiponectin concentration were noted in the Mediterranean diet group compared with the control group. In a randomised controlled trial lasting 2 years, 180 subjects with the metabolic syndrome were assigned either to a Mediterranean-style diet (instructions were provided on increasing daily consumption of whole grains, fruit, vegetables, nuts and olive oil) or to a control group (prudent diet, with same macronutrient composition as the Mediterranean diet) ${ }^{(284)}$. After 2 years, body weight decreased more in the intervention group and inflammatory markers (CRP, IL-6, IL-7 and IL-18) decreased and endothelial function improved, compared with the control group. Interestingly, even after controlling for weight loss, the inflammatory markers declined more in subjects following the Mediterranean-style diet. In other related studies involving individuals with the metabolic syndrome, a consistent reduction in CRP concentration in the intervention group receiving the Mediterranean diet has been shown ${ }^{(291,292)}$. In the Prevención con Dieta Mediterránea study, 772 asymptomatic subjects at high cardiovascular risk (diabetes or more than three CHD risk factors) were randomly assigned to a low-fat diet or one of two Mediterranean diets ${ }^{(293)}$. Those allocated to the Mediterranean diets received nutritional education and either free virgin olive oil, or free nuts for 3 months. Both Mediterranean diets were beneficial in terms of significant reductions in serum IL-6, sICAM-1 and sVCAM-1 concentrations, while CRP concentration was reduced only in the Mediterranean diet supplemented with olive oil. Taken together, the results from these often large intervention studies strongly suggest that Mediterranean diets can lead to reductions in chronic lowgrade inflammation and improvement in endothelial function, thereby offering cardioprotective effects.

Vegetarian diets. Observational studies have compared vegetarian with non-vegetarian diets in healthy subjects in relation to inflammatory markers. Dietary patterns consistent with vegetarianism were associated with lower concentrations of markers of chronic low-grade inflammation and endothelial function when compared with non-vegetarian diets ${ }^{(294-296)}$. In one study comparing a group of thirty Taoist adults who had been vegetarian for 5-55 years (average 22 years) with a group of thirty age- and sex-matched non-Taoist adults consuming a non-vegetarian diet, lower CRP concentrations were noted in the former group ${ }^{(295)}$. Specifically, plasma CRP concentration averaged 0.77 and $1.30 \mathrm{mg} / 1$ in vegetarians and non-vegetarians, respectively. Another study with a similar design comparing vegetarians with non-vegetarians also found that the average CRP concentration was significantly lower in the vegetarian group $(0.72 v .1 .62 \mathrm{mg} / \mathrm{l})^{(294)}$. Similarly, lower levels of the adhesion molecules sICAM-1 and sE-selectin have been reported in those following a vegetarian $v$. a non-vegetarian diet ${ }^{(296)}$. Thus, these cross-sectional studies suggest that a vegetarian-style diet can lead to lower chronic low-grade inflammation than an omnivorous diet. However, it is important to recognise that vegetarians may differ from non-vegetarians in aspects of lifestyle other than diet such as physical activity, smoking behaviour and socio-economic class. Studies considering vegetables and fruits are described in section 'Vegetables and fruits'.

Eating patterns. The healthy eating index (HEI) was developed by the US Department of Agriculture, based on the Dietary Guidelines for Americans and the Food Guide Pyramid $^{(297)}$. It has ten subcomponents: grains, fruits, vegetables, dairy, meats, fats, saturated fat, cholesterol, $\mathrm{Na}$ and dietary variety. Individuals are assigned scores for each of the ten components (from 0 to 10) based on their typical intakes; the maximum value for the HEI being 100. Recently, the HEI was revised as the Alternate HEI (AHEI) to focus on healthier items in the Food Guide Pyramid food groups ${ }^{(298)}$ such as protein source, ratio of polyunsaturated:saturated fats and cereal fibre, as well as cis- $v$. trans-fat. Additionally, moderate alcohol consumption and long-term multivitamin use are also considered in the AHEI. The Diet Quality Index (DQI) is a composite score of an overall healthy diet that reflects an individual's adherence to the eight diet and health recommendations of the National Academy of Science. The revised DQI (DQI-R) is based on similar guidelines but also includes Fe and $\mathrm{Ca}$ intakes ${ }^{(299)}$.

Using the data from NHANES III on a representative sample of the US population, Ford et al. ${ }^{(300)}$ found a negative correlation between the HEI and CRP concentration after adjustment for several CVD risk factors including BMI and waist:hip ratio; when stratified by sex this finding was significant in women only. The authors noted that this association was primarily driven by grain consumption. The authors speculated that because HEI score was determined based on dietary intake data collected by a $24 \mathrm{~h}$ recall, the possibility of misclassification of individuals with respect to HEI status could have attenuated the associations. In a study by Fung et $a l^{(285)}$, the association of several diet-quality scores, such as the HEI, AHEI, DQI-R and an alternate Mediterranean diet index with various markers of inflammation, was evaluated in a subsample of the Nurses' Health Study ( $n 690$ healthy women). The key findings were that the AHEI and alternate Mediterranean diet index scores were negatively associated with CRP, IL-6, sE-selectin and sICAM-1 concentrations, and that these associations persisted upon adjustment for potential confounding variables including BMI. In contrast, the HEI and 
DQI-R did not correlate with any of the inflammatory markers when potential confounders were taken into consideration in the regression models ${ }^{(285)}$. A more recent study evaluated the impact of the AHEI among 1922 women from the Nurses' Health Study (62\% of whom were overweight) who had no history of diabetes or CVD on plasma inflammatory marker concentrations ${ }^{(301)}$. After adjustment for age and energy intake, women with the highest adherence to the AHEI had $24 \%$ higher median total adiponectin and 32\% higher median high-molecular-weight adiponectin concentrations, as well as 16\% lower resistin, 41\% lower CRP and 19\% lower sE-selectin concentrations than did women with the lowest adherence to the AHEI. These associations remained significant after adjustment for potential confounders. Inverse associations between the AHEI and CRP, sTNFR2, IL-6, sICAM1 and sVCAM-1 concentrations were evident, but they did not remain significant after adjustment for BMI. In a small crosssectional study involving 114 'apparently healthy' overweight or obese postmenopausal women, Boynton et $a l{ }^{(302)}$ found little evidence of an association between dietary quality, as measured by the HEI and DQI, and markers of inflammation (CRP and SAA). Marginal associations were noted between the DQI and these inflammatory markers. These associations were, however, attenuated and no longer significant, after adjusting for adiposity (percentage of body fat or BMI), suggesting that the decrease in CRP or SAA concentration seen with a higher-quality diet is most probably mediated by obesity. The authors speculated that consuming a healthier diet may lead to decreased adiposity, which in turn could result in less low-grade inflammation.

Several studies have examined the relationship between consuming a 'prudent diet' and markers of low-grade inflammation. In one study, in 732 healthy women from the Nurses' Health Study aged 43-69 years, a prudent pattern was characterised by higher intakes of fruit, vegetables, legumes, fish, poultry and whole grains, and a Western pattern was characterised by higher intakes of red and processed meats, sweets, desserts, French fries and refined grains ${ }^{(303)}$. The prudent pattern was inversely associated with plasma concentrations of CRP and sE-selectin after adjustment for age, BMI, physical activity, smoking status and alcohol consumption. The Western pattern showed a positive relationship with CRP, IL-6, sE-selectin, sICAM-1 and sVCAM-1 after adjustment for all confounders except BMI; with further adjustment for BMI, the coefficients remained significant for CRP, sE-selectin, sICAM-1 and sVCAM-1.Using data from the Nurses' Health Study, Schulze et al. ${ }^{(304)}$ identified a dietary pattern that was significantly associated with increased concentrations of CRP, IL-6, sTNFR2, sE-selectin, sICAM-1 and sVCAM-1. This pattern, which was high in sugar-sweetened soft drinks, refined grains, diet soft drinks and processed meat but low in wine, coffee, cruciferous vegetables and yellow vegetables, was also associated with an increased risk of diabetes. Most recently, Hoebeeck et al. ${ }^{(305)}$ evaluated the relationship between adherence to food-based dietary guidelines and inflammatory markers among 2524 healthy Belgian men and women aged 35-55 years. The dietary index consisted of three subscores (dietary quality, diversity and equilibrium) according to adherence to the Flemish food-based dietary guidelines, using data from a semiquantitative FFQ. Higher dietary scores were inversely associated with IL-6 concentration and leucocyte numbers in the bloodstream. Nettleton et al. $^{(306)}$ examined relationships between dietary patterns and markers of inflammation and endothelial activation among 5089 non-diabetic participants in the Multi-Ethnic Study of Atherosclerosis. In this study, four dietary patterns were derived by using factor analysis. The fats and processed meats pattern (fats, oils, processed meats, fried potatoes, salty snacks and desserts) was positively associated with CRP and IL- 6 concentrations. The beans, tomatoes and refined grains pattern (beans, tomatoes, refined grains and high-fat dairy products) was positively related to sICAM-1 concentration. In contrast, the whole grains and fruit pattern (whole grains, fruit, nuts and green leafy vegetables) was inversely associated with CRP, IL-6 and sICAM-1 concentrations, while the vegetables and fish pattern (fish and dark-yellow, cruciferous and other vegetables) was inversely related to IL-6 concentration. There are few such studies using data from outside of the USA or Europe. A cross-sectional study of 486 healthy Iranian women aged 40-60 years identified dietary patterns by factor analysis and related these to circulating markers of inflammation ${ }^{(307)}$. The healthy dietary pattern (high in fruits, vegetables, tomatoes, poultry, legumes, tea, fruit juices and whole grains) was inversely related to plasma CRP, sE-selectin and sVCAM-1 concentrations after controlling for potential confounders; with further adjustment for BMI and waist circumference, the associations remained significant for CRP and SVCAM-1. In contrast, the Western dietary pattern score (high in refined grains, red meat, butter, processed meat, high-fat dairy, sweets and desserts, pizza, potatoes, eggs, hydrogenated fats and soft drinks) was positively related to CRP, SAA, IL-6, sICAM-1 and sVCAM-1 concentrations; however, after additional control for BMI and waist circumference, the associations remained significant only for SAA and IL-6.The traditional dietary pattern (high in refined grains, potatoes, tea, whole grains, hydrogenated fats, legumes and casserole) was positively associated with the plasma IL- 6 concentration after controlling for BMI and waist circumference. Nanri et $a l^{(308)}$ investigated the relationship between dietary patterns and circulating CRP concentration in 7802 Japanese men and women with $\mathrm{CRP}<3 \mathrm{mg} / \mathrm{l}$. The dietary patterns were derived from principal component analysis of the frequency of consumption of forty-nine food items ascertained by the FFQ. The following four dietary patterns were identified: healthy, high-fat, seafood and Westernised breakfast patterns. The healthy dietary pattern, characterised by high intakes of vegetables, fruits, soya products and fish, was inversely related to CRP concentrations, even after adjustment for age, BMI, smoking, alcohol consumption and physical activity in both men and women. Neither the high-fat dietary pattern nor the Westernised breakfast pattern was related to CRP concentrations. Most recently, the relationship between two dietary patterns (Healthy and Western), which were derived by principal component analysis using data collected by a FFQ from subjects in the Atherosclerosis Risk in Communities 
Study, and markers of inflammation has been described ${ }^{(309)}$. The measures of inflammation were quantified by flow cytometry in fresh whole blood from 1101 white adults. After multivariable adjustment, monocyte LPS receptor (CD14), monocyte TLR-2 and platelet glycoprotein IIb (CD41) showed inverse associations with the healthy dietary pattern. In contrast, the Western dietary pattern was positively associated with CD41 and platelet-granulocyte aggregates. Taken together, these studies suggest that healthy eating patterns are associated with lower concentrations of markers of chronic low-grade inflammation.

\section{Whole foods}

Whole grains/refined grains. Published studies have so far investigated a narrow and low range of whole grain intakes, which limits the interpretation of associations between whole grain intake and markers of low-grade chronic inflammation. Observational studies (Table 3$)^{(300,310-314)}$ including data from NHANES III have suggested that a high intake of whole grain is inversely associated with plasma CRP concentration (quintile (Q) $1<3.5$ servings/d, Q5 $>9.7$ servings/d) ${ }^{(300)}$. In contrast, Jensen et $a l .{ }^{(310)}$ reported no association between a moderate whole grain intake (Q1 $8.2 \mathrm{~g} / \mathrm{d}$, Q $543.8 \mathrm{~g} / \mathrm{d}$ ) and markers of inflammation (CRP, IL- 6 and fibrinogen) in the Health Professionals' Follow-Up Study. However, bran intake correlated inversely with CRP concentration and germ intake with IL-6 concentration $^{(310)}$. Data from the Nurses' Health Study showed lower CRP and sTNFR2 concentrations in diabetic women with a moderate intake of whole grain compared with those with a low intake ${ }^{(314)}$. In another study with diabetic males ( $n$ 780; from the Health Professionals' Follow-Up Study), Qi et ${ }^{\left({ }^{(315)}\right.}$ showed that cereal fibre was positively associated with adiponectin concentration after controlling for a number of confounding factors. A higher intake of whole grain (low 0.8 servings/d, high $2 \cdot 1$ servings/d) within a Mediterranean diet in diabetic women was associated with increased adiponectin concentration ${ }^{(312)}$. Data from the Multi-Ethnic Study of Atherosclerosis (MESA) showed a higher whole grain intake (Q1 0.02 servings/d, Q5 1.39 servings/d) to be associated with lower CRP concentration in elderly subjects ${ }^{(311)}$

Evidence from intervention studies (Table 4) (111,316-320) $^{(2)}$ includes a study whereby overweight and obese subjects consumed a hypoenergetic diet with or without whole-grain foods. CRP concentration decreased by 38\% (CRP at baseline $5.9-6.0 \mathrm{mg} / \mathrm{l})$ in the whole-grain group independent of the observed weight loss ${ }^{(319)}$. However, plasma concentrations of IL- 6 and TNF- $\alpha$ and PAI- 1 activity did not change within the 12-week-study period. Based on the outcome of this study, the authors concluded that the changes seen in CRP concentration with a whole-grain diet were not part of a systemic anti-inflammatory effect ${ }^{(319)}$. In a study from Sweden with a similar design but only a 6-week intervention (wholegrain $v$. refined-grain products), the whole grain intake was not associated with changes in the concentrations of CRP and IL- 6 or in PAI- 1 activity of moderately overweight subjects $^{(316)}$. Baseline CRP concentration $(2 \cdot 03-2.86 \mathrm{mg} / \mathrm{l})$ and BMI were much lower than in the previous study, which may explain the different outcomes between these studies. Recent intervention trials with whole grain $(60-120 \mathrm{~g} / \mathrm{d})$ or wholemeal intake (30-40 g/d) and comparable CRP baseline concentrations also did not observe any changes in plasma markers of inflammation ${ }^{(317,320)}$. In the study by Brownlee et $a l .{ }^{(317)}$, whole-grain products did not substitute refinedgrain products but were consumed in addition to the refined products. Bioprocessing of whole wheat affected the antiinflammatory potential in a human intervention study when compared with unprocessed whole wheat ${ }^{(321)}$. Bioprocessing modulated the ratio of pro-inflammatory:anti-inflammatory cytokines during $24 \mathrm{~h}$ after the intake of $300 \mathrm{~g}$ whole wheat bread. This study suggests that subtle changes in the processing of whole grains may be relevant for their anti-inflammatory activity, which could in part explain the contradictory findings of the human intervention studies.

Replacing a refined-wheat flour pizza by a similar pizza prepared from whole-wheat flour resulted in a decreased postprandial concentration of the pro-inflammatory cytokine IL-18 in both non-diabetic and diabetic subjects ${ }^{(318)}$.

In summary, whole grain intake appears to inversely associate with markers of low-grade inflammation. Processing status of whole-grain products should be more precisely defined in future studies. Potential mechanisms still have to be elucidated, as well as the active constituents which may include dietary fibre, minerals, vitamins and phytochemicals such as lignans and phenolic acids.

Vegetables and fruits. A number of cross-sectional studies have investigated the association between vegetable and fruit intake and biomarkers of inflammation (Table 5) ${ }^{(288,322-330)}$. Study participants included healthy, normal-weight adults as well as overweight/obese adults with associated diseases. Each study applied different criteria to stratify the intake of vegetables and fruits which makes it difficult to compare the outcomes. Of the ten cross-sectional studies, seven reported an inverse association between a high intake of vegetables and fruits, either in combination or alone, and blood CRP concentrations $^{(322-325,327-329)}$. In one study, no association with CRP was observed ${ }^{(288)}$, while in a second study, an inverse association was seen in men but not in women ${ }^{(327)}$. For the other biomarkers reported, the outcome is less consistent. A recent study reported that a high intake of vegetables and fruits was associated with a lower peripheral blood mononuclear cell gene expression for several pro-inflammatory cytokines and adhesion molecules ${ }^{(326)}$. An important observation is that besides the quantity of total intake of vegetables and fruits, the variety consumed in a given time has a significant impact on these biomarkers. A high number of varieties of vegetables and fruits were inversely correlated with blood CRP levels ${ }^{(322)}$. This suggests that plant-specific constituents of vegetables and fruits such as the phytochemicals may contribute to the anti-inflammatory activities.

A number of intervention studies have investigated the impact of vegetables and fruits in total or of specific varieties on biomarkers of inflammation (Table 6) ${ }^{(331-346)}$. Of the four studies focusing on vegetables and fruits as a food group, three reported a reduction in blood concentrations of different biomarkers of inflammation ${ }^{(318,342,345)}$, while one study did 
not find any significant effect ${ }^{(336)}$. In twelve studies with a specific focus on a single variety of vegetable or fruit, inconsistent results were reported. Most studies have used fruits or fruit extracts high in polyphenols. Results from such studies suggest an anti-inflammatory effect; however, mostly, only a single biomarker was affected, and never the complete set of inflammation biomarkers investigated ${ }^{(331-334,337-340,346)}$. In conclusion, current evidence for specific effects of single vegetable and fruit varieties is not convincing, while a high overall intake of vegetables and fruits seems to be associated with a lower state of inflammation.

Soya. Several randomised controlled intervention trials have shown that supplementation with different quantities of soya protein did not affect markers of inflammation (CRP, IL-6, IL-18, sICAM-1, sVCAM-1 and sE-selectin) $(\text { Table } 7)^{(345,347-364)}$. Variations in soya protein isoflavone contents did not modulate the outcome in these studies. In one study, reduced plasma CRP, TNF- $\alpha$ and IL-18 concentrations were reported in postmenopausal Iranian women with the metabolic syndrome consuming soya nuts but not soya protein ${ }^{(347)}$. The major difference between soya protein and soya nuts (comparable contents of isoflavones) was a much higher content of PUFA in the soya nuts, suggesting that rather than soya-specific constituents, the increased intake of PUFA may be responsible for the reduction in biomarkers of inflammation $^{(347)}$. The same group investigated in diabetic patients with nephropathy the anti-inflammatory effect of textured soya protein compared with animal protein after 4 years of intake. Consumption of soya protein significantly lowered plasma concentrations of $\mathrm{CRP}^{(348)}$. In another study investigating soya nuts in normotensive and hypertensive postmenopausal women, no effect on CRP, IL-6, sICAM-1 or matrix metalloproteinase-9 concentrations was observed compared with the control diet ${ }^{(361)}$. However, in hypertensive women, levels of sVCAM-1 were significantly lower after soya nut consumption $^{(361)}$. Again, the increased PUFA intake may have caused this effect. Long-term soya exposure did not affect CRP, IL-6, leptin and adiponectin concentrations in postmenopausal women $^{(357)}$, or CRP, IL-6, leptin, adiponectin, MCP-1 or MIP-1 $\beta$ concentrations in men ${ }^{(365)}$. Short-term soya intervention in postmenopausal women with or without exerciseinduced inflammation had no effect on IL- $1 \beta$, IL- 6 or TNF- $\alpha$ concentrations ${ }^{(349,350)}$. Among men with prostate cancer undergoing androgen deprivation therapy, soya intervention had no effect on the concentrations of several inflammatory markers ${ }^{(366)}$. Overall, these data suggest that soya protein does not affect markers of inflammation, that soyabean processing may affect the anti-inflammatory potential of soyabean constituents and that the health status of the study subjects might determine the anti-inflammatory efficacy of specific foods.

Nuts. To date, only few studies have investigated the effect of nuts on inflammatory markers. The MESA reported that a high intake of nuts and seeds ( $\geq 5$ times/week) compared with a low intake (rarely or no consumption) was associated with lower plasma concentrations of CRP, IL-6 and fibrinogen ${ }^{(367)}$. In contrast, data from the Nurses' Health Study suggest that nut intake is not associated with inflammatory

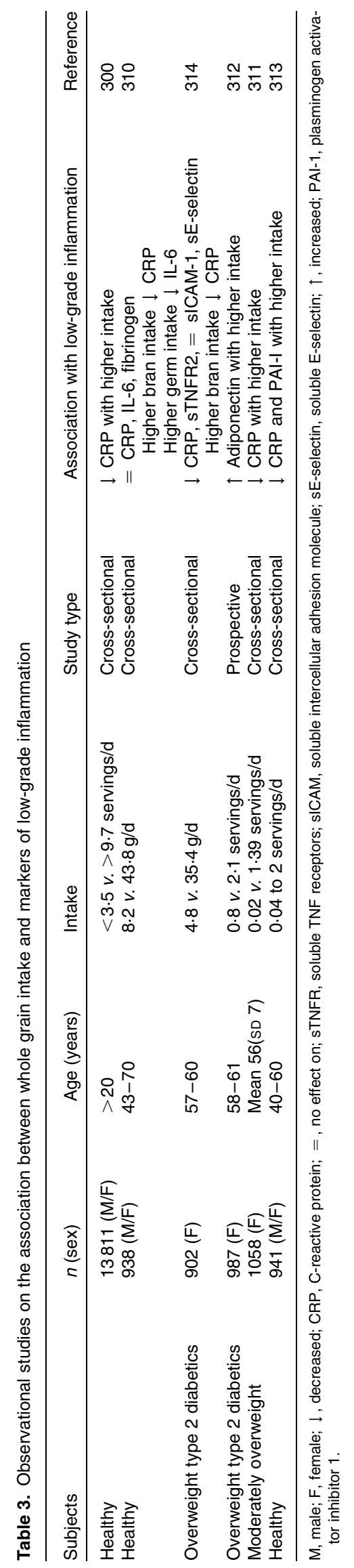




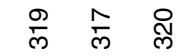
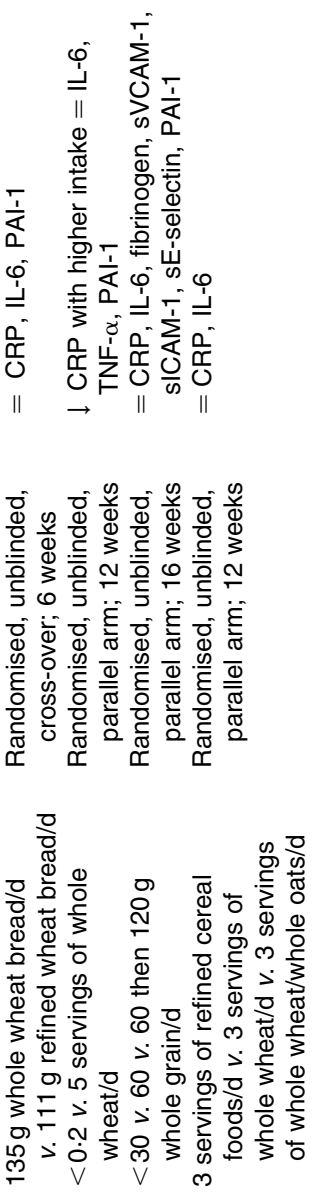

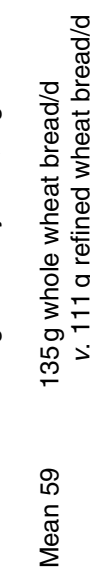

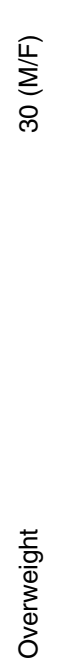

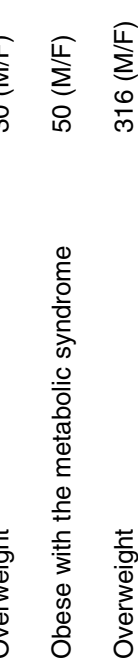

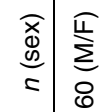

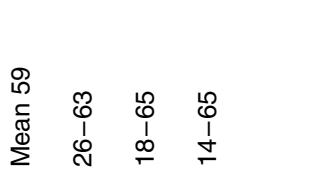$$
\sum_{\substack{I \\ 0}}^{\substack{N \\ \text { N }}}
$$

markers including sTNFR2, CRP, fibrinogen, sICAM-1 and sE-selectin ${ }^{(368)}$. An 8-week intervention with walnuts or cashews $(63-108 \mathrm{~g} / \mathrm{d})$ in subjects with the metabolic syndrome observed no effect on serum CRP concentrations ${ }^{(360)}$. A pistachio intervention $(60-100 \mathrm{~g} / \mathrm{d}$ providing about $20 \%$ of total energy) for 4 weeks in healthy young subjects had no effect on plasma CRP or TNF- $\alpha$ concentrations, while the concentration of IL-6 was significantly reduced ${ }^{(369)}$. A recent intervention study with two doses of almonds (10 and 20\% of total energy) in healthy adults reported reduced serum concentrations of sE-selectin and CRP, but IL- 6 and fibrinogen were not affected ${ }^{(370)}$. No clear dose-response relationships were observed. In contrast, an earlier study in hyperlipidaemic subjects providing two doses of almonds did not observe an effect on CRP concentrations ${ }^{(356)}$. A systematic review on walnut consumption and inflammatory markers also reported inconsistent results for plasma CRP, while walnuts added to a Mediterranean diet resulted in significantly lower sVCAM-1 concentrations $^{(371)}$. This suggests that rather than a general anti-inflammatory effect, walnuts may exert anti-inflammatory effects primarily in the endothelium. Major contributors to any anti-inflammatory activity of nuts are likely to include PUFA, $\mathrm{Mg}$ and phytochemicals including ellagic acid(372).

Fish. Increased frequency of fish consumption was associated with lower CRP and IL- 6 concentrations in a cohort of 727 adults in the USA ${ }^{(373)}$. sICAM-1 and sE-selectin concentrations also decreased but the effect of fish consumption was weaker than what was observed for CRP and IL-6. sTNFR2 concentration was not associated with fish consumption. A study in 379 adults in Denmark reported a lack of association between fish consumption and CRP concentration, even though the subjects displayed a wide range of intakes including very high intakes (e.g. over $30 \%$ of subjects ate fish more than once per d) ${ }^{(374)}$. Data from 5037 adults in the NHANES showed no association of fish consumption with CRP concentration after adjusting for a range of confounders ${ }^{(375)}$. In this study, $25 \%$ of subjects consumed fish more than 3 times per month. Most recently, fish consumption among a cohort of 4077 Australian adults was reported not to be associated with CRP concentration before or after adjustment for confounders ${ }^{(376)}$. In contrast to these findings, a study in 3102 Greek adults reported that fish consumption was 'dose-dependently' associated with lower CRP, IL- 6 , TNF- $\alpha$ and SAA concentrations and white blood cell counts; individuals consuming $>300 \mathrm{~g}$ fish/week ( $n$ 259; $9 \%$ ) had significantly lower concentrations of CRP ( $-33 \%)$, IL-6 $(-33 \%), \quad$ TNF- $\alpha(-21 \%)$, SAA $(-28 \%)$ and leucocytes $(-4 \%)$ than seen in individuals not consuming fish ( $n 319$; $11 \%)^{(377)}$. It is possible that the observations of Zampelas et al. may be due to greater fish consumption than in the other populations studied and/or to the type of fish consumed. No studies have discriminated between consumption of lean $v$. fatty fish. A 4-week intervention study with herring $(150 \mathrm{~g} / \mathrm{d}$, $5 \mathrm{~d} /$ week) in overweight and obese adults in Sweden ( $n$ 13) reported a trend towards lower CRP concentration compared with the concentration seen when the subjects consumed a reference diet, but the $30 \%$ reduction in CRP was not significant ${ }^{(378)}$. The lack of statistical significance of the effect seen may be due to the small sample size. 


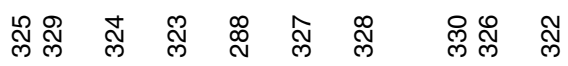

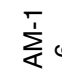<smiles>[C]1[C]C=C1</smiles>

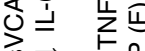

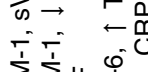

$\sum^{T} \sum^{+} \sum^{-1}$

$\sum_{\substack{0 \\ 0}} \stackrel{1}{=} \|$ की

응

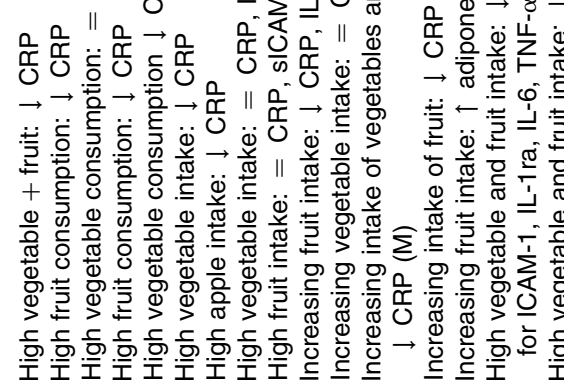

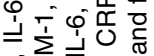

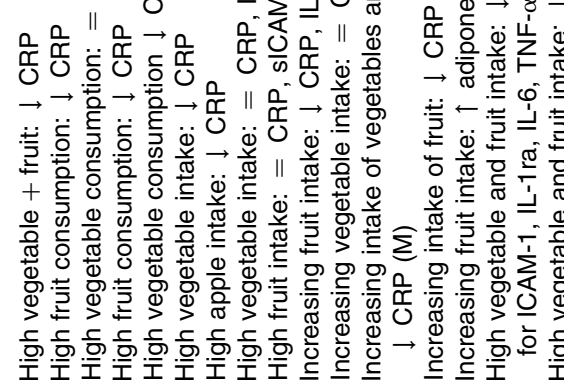

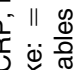

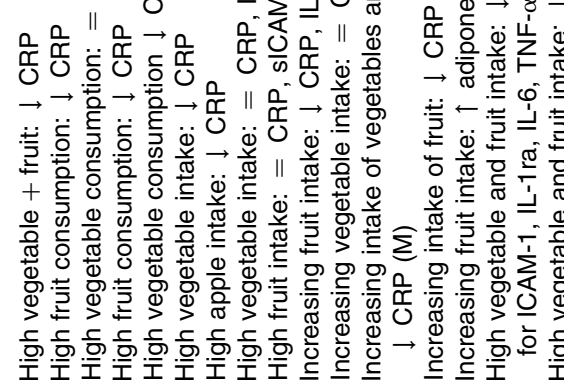

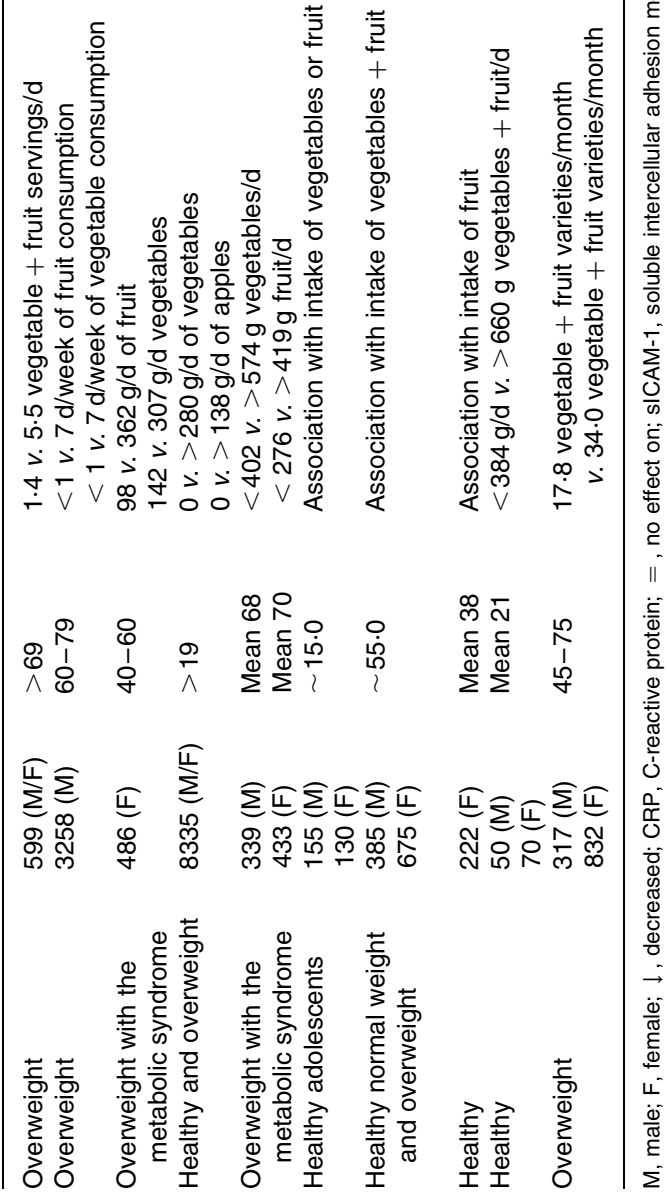

Tea. A cross-sectional study from Japan reported no relationship between green tea consumption and the concentrations of several inflammatory markers ${ }^{(379)}$, while a crosssectional study from Belgium detected significantly lower CRP and SAA concentrations in regular tea drinkers after multivariate analysis ${ }^{(380)}$. The effect of tea consumption (green or black tea) has been investigated in several small intervention studies usually with a fairly short duration (Table 8) ${ }^{(381-389)}$. Most studies indicate that tea consumption has no significant effect on the markers of inflammation reported ${ }^{(382-386,388,389)}$. However, a longer supplementation period of 6 weeks with black tea in healthy non-smoking men reduced plasma CRP concentrations significantly ${ }^{(380,387)}$. Overall, there are no consistent data that tea consumption (black or green) has a beneficial effect on inflammatory status, but studies of longer duration than those currently reported need to be performed.

The modest or even absent effects of tea on inflammatory and oxidative stress markers in vivo are surprising in view of the potent inhibitory effects of tea components such as catechins on the expression of pro-inflammatory mediators in vitro ${ }^{(390-392)}$. This may be due to the fact that the majority of tea catechins undergo methylation, glucuronidation and sulfation during uptake which may limit bioavailability ${ }^{(393)}$. Furthermore, studies in vitro have often used catechin concentrations $>10 \mu \mathrm{M}$, whereas plasma concentrations of catechins after ingestion of tea rarely exceed $1 \mu \mathrm{M}^{(394)}$.

Coffee. Habitual coffee consumption was analysed for association with markers of low-grade inflammation in crosssectional epidemiological studies which yield conflicting results (Table 9) ${ }^{(304,395-398)}$. Consumption of decaffeinated coffee was not associated with changes in systemic levels of soluble adhesion molecules, but CRP concentrations were lower in decaffeinated coffee drinkers in one of the two studies ${ }^{(396)}$. The contradictory findings from observational studies may reflect the fact that coffee contains a mixture of bioactives with divergent effects on physiology ${ }^{(399-403)}$. A recent intervention study providing two doses of coffee ( 4 and 8 cups of filtered coffee/d) in the same individuals confirmed in part the observations from the cross-sectional studies. While IL-18 (decreased) and adiponectin (increased) were significantly modulated by coffee consumption, concentrations of CRP, leptin, SAA, IL-6, MIF and IL-1ra were not affected $^{(404)}$. No dose-response relationship was seen in this study. Plasma caffeine concentrations were positively associated with plasma adiponectin concentrations ${ }^{(404)}$. Taken together, the available data do not allow a firm conclusion as to whether coffee consumption modulates lowgrade inflammation.

Cocoa. Cocoa has a high content of monomeric (epicatechin and catechin) and oligomeric (procyanidin) flavanols ${ }^{(405-407)}$. These latter polymeric fractions are present in higher concentrations/amounts in cocoa compared with other flavanol-rich foods such as red wine or green tea ${ }^{(408,409)}$. Thus, certain cocoa-based products are rich in flavanols $^{(410,411)}$, some of which have been found in model systems to possess potential anti-inflammatory activities. However, the effect of cocoa flavanols and their related procyanidins appears to be related to the degree of polymerisation, 
Table 6. Intervention studies investigating the effect of vegetable and fruit intake on markers of low-grade inflammation

\begin{tabular}{|c|c|c|c|c|c|c|}
\hline Subjects & $n(\operatorname{sex})$ & Age (years) & Intake & Study design and duration & Effect on low-grade inflammation & Reference \\
\hline Healthy & $\begin{array}{l}13(\mathrm{M}) \\
12(\mathrm{~F})\end{array}$ & $23-40$ & $\begin{array}{l}\text { High-fat meal } \\
\quad v \text {. high-fat meal }+400 \mathrm{~g} \text { of vegetables }\end{array}$ & Single meal, short term & $\downarrow$ PAl-1 & 335 \\
\hline Healthy & $\begin{array}{l}6(\mathrm{M}) \\
6(\mathrm{~F})\end{array}$ & Mean 22 & $500 \mathrm{ml} / \mathrm{d}$ of high-pressure orange juice & $\begin{array}{l}\text { Randomised, open label, } \\
\text { uncontrolled; } 14 \mathrm{~d}\end{array}$ & $=\mathrm{CRP}$ & 343 \\
\hline Healthy & $\begin{array}{l}6(\mathrm{M}) \\
6(\mathrm{~F})\end{array}$ & Mean 22 & $500 \mathrm{ml} / \mathrm{d}$ of vegetable soup & $\begin{array}{l}\text { Randomised, open label, } \\
\text { uncontrolled; } 14 \mathrm{~d}\end{array}$ & $\begin{array}{l}\downarrow \mathrm{MCP}-1, \mathrm{PGE}_{2} \\
=\mathrm{TNF}-\alpha, \mathrm{IL}-6, \mathrm{IL}-1\end{array}$ & 342 \\
\hline Healthy & $\begin{aligned} 5(\mathrm{M}) \\
13(\mathrm{~F})\end{aligned}$ & $19-52$ & $\begin{array}{l}196 \mathrm{~g} / 10 \mathrm{MJ} v .810 \mathrm{~g} / 10 \mathrm{MJ} \text { of } \\
\text { vegetables + fruit }\end{array}$ & $\begin{array}{l}\text { Randomised, controlled, } \\
\text { parallel; } 6 \text { weeks }\end{array}$ & $=\mathrm{CRP}$, sICAM-1, sP-selectin & 336 \\
\hline $\begin{array}{l}\text { Obese pre- and } \\
\text { postmenopausal women }\end{array}$ & $\begin{array}{l}24 \text { (pre-) } \\
20 \text { (post-) }\end{array}$ & $\begin{array}{l}\text { Mean } 38 \text { (pre-) } \\
\text { Mean } 58.5 \text { (post-) }\end{array}$ & $36 \mathrm{~g} / \mathrm{d}$ of lyophilised grape powder & $\begin{array}{l}\text { Randomised, controlled, } \\
\text { parallel; } 4 \text { weeks }\end{array}$ & $\downarrow$ TNF- $\alpha,=$ IL-6, CRP & 346 \\
\hline Healthy & $63(\mathrm{M})$ & $31-32$ & 2,5 or 8 servings/d of vegetables + fruit & $\begin{array}{l}\text { Randomised, open label, } \\
\text { parallel; } 4 \text { weeks }\end{array}$ & $\begin{array}{l}\downarrow \text { CRP with high intake } \\
=\mathrm{IL}-12, \mathrm{TNF}-\alpha\end{array}$ & 345 \\
\hline Overweight & $\begin{array}{l}25(\mathrm{M}) \\
29(\mathrm{~F})\end{array}$ & Mean 49 & $\begin{array}{l}\text { Garlic powder }(2.1 \mathrm{~g} / \mathrm{d})(=5.2 \mathrm{~g} \text { fresh garlic) } \\
\quad \text { v. placebo }\end{array}$ & $\begin{array}{l}\text { Randomised, double blind, } \\
\text { controlled; } 3 \text { months }\end{array}$ & $\begin{array}{l}=\text { CRP, TNF- } \alpha, \text { sICAM-1, } \\
\text { sVCAM- } 1 \text {, sE-selectin, } \\
\text { fibrinogen }\end{array}$ & 344 \\
\hline Haemodialysis patients & $\begin{array}{l}4(\mathrm{M}) \\
6(\mathrm{~F})\end{array}$ & & $100 \mathrm{ml} / \mathrm{d}$ of red grape juice & $\begin{array}{l}\text { Randomised, open label, } \\
\text { uncontrolled; } 3 \text { weeks }\end{array}$ & $\begin{array}{l}\downarrow \mathrm{MCP}-1 \\
=\mathrm{CRP}, \text { sICAM }-1, \text { sVCAM }-1\end{array}$ & 332 \\
\hline Healthy & $18(\mathrm{M} / \mathrm{F})$ & $45-61$ & $280 \mathrm{~g} / \mathrm{d}$ of cherries & $\begin{array}{l}\text { Randomised, open label, } \\
\text { uncontrolled; } 28 \mathrm{~d}\end{array}$ & $\begin{array}{l}\downarrow \text { CRP, RANTES } \\
=\mathrm{IL}-6\end{array}$ & 339 \\
\hline Healthy & $\begin{array}{l}61(\mathrm{~F}) \\
59(\mathrm{M})\end{array}$ & $40-74$ & $\begin{array}{l}300 \mathrm{mg} / \mathrm{d} \text { of anthocyanins ( }=100 \text { fresh } \\
\text { bilberries) } v \text {. placebo }\end{array}$ & $\begin{array}{l}\text { Randomised, controlled, } \\
\text { parallel; } 3 \text { weeks }\end{array}$ & $\begin{array}{l}\downarrow \text { RANTES, IL-8, IFN- } \alpha \\
=\text { CRP, IL-1, IL-6, TNF- } \alpha, \text { MCP-1 }\end{array}$ & 337 \\
\hline Healthy & $180(\mathrm{M} / \mathrm{F})$ & $19-50$ & $\begin{array}{l}28 \mathrm{~g} / \mathrm{d} \text { of a frozen seabuckthorn } \\
\text { puree } v \text {. placebo }\end{array}$ & $\begin{array}{l}\text { Randomised, controlled, } \\
\text { parallel; } 3 \text { months }\end{array}$ & $\downarrow \mathrm{CRP}$ & 340 \\
\hline $\begin{array}{l}\text { Patients with } \\
\text { peripheral arterial disease }\end{array}$ & $47(\mathrm{M} / \mathrm{F})$ & $57-61$ & $\begin{array}{l}250 \mathrm{ml} \text { orange juice }+250 \mathrm{ml} \text { blackcurrant } \\
\text { juice } v \text {. placebo }\end{array}$ & $\begin{array}{l}\text { Randomised, open label, } \\
\text { uncontrolled; } 28 \mathrm{~d}\end{array}$ & $\begin{array}{l}\downarrow \text { CRP } \\
=\mathrm{IL}-6, \mathrm{PAI}-1\end{array}$ & 334 \\
\hline $\begin{array}{l}\text { Healthy postmenopausal } \\
\text { women }\end{array}$ & 52 & Mean 58 & $\begin{array}{l}500 \mathrm{mg} / \mathrm{d} \text { of elderberry extract }(=25 \mathrm{~g} / \mathrm{d} \\
\text { elderberries) } v \text {. placebo }\end{array}$ & $\begin{array}{l}\text { Randomised, controlled, } \\
\text { parallel; } 12 \text { weeks }\end{array}$ & $\begin{array}{l}=\text { CRP, IL-6, TNF- } \alpha, \text { sTNFR1 } \\
\text { and 2, RANTES }\end{array}$ & 333 \\
\hline Metabolic syndrome & $48(\mathrm{M} / \mathrm{F})$ & Mean $52(\mathrm{M}) / 48(\mathrm{~F})$ & $\begin{array}{l}960 \mathrm{ml} / \mathrm{d} \text { water with } 50 \mathrm{~g} / \mathrm{d} \text { of } \\
\text { freeze-dried blueberries }(=350 \mathrm{~g} / \mathrm{d} \text { fresh } \\
\text { blueberries) v. } 960 \mathrm{ml} / \mathrm{d} \text { water }\end{array}$ & $\begin{array}{l}\text { Randomised, controlled, } \\
\text { parallel; } 8 \text { weeks }\end{array}$ & $\begin{array}{l}=\mathrm{CRP}, \text { sICAM-1, sVCAM-1, } \\
\text { IL-6, adiponectin }\end{array}$ & 331 \\
\hline Elevated risk for CVD & $62(\mathrm{M} / \mathrm{F})$ & Mean 53 & $\begin{array}{l}330 \mathrm{ml} / \mathrm{d} \text { of bilberry juice diluted to } 1 \text { litre of } \\
\text { water } v \text {. water alone }\end{array}$ & $\begin{array}{l}\text { Randomised, controlled, } \\
\text { parallel; } 4 \text { weeks }\end{array}$ & $\begin{array}{l}\downarrow \text { CRP, IL-6, IL-15, MIG } \\
=\text { WBC count, IL-1, IL-1ra, } \\
\text { MCP- } 1 \text {, RANTES } \\
\uparrow \text { TNF- } \alpha\end{array}$ & 338 \\
\hline Healthy overweight & $24(\mathrm{M})$ & Mean 56 & $500 \mathrm{ml} / \mathrm{d}$ of orange juice $v$. control drink & $\begin{array}{l}\text { Randomised, controlled, } \\
\text { parallel; } 4 \text { weeks }\end{array}$ & $=\mathrm{CRP}, \mathrm{IL}-6, \mathrm{sICAM}-1, \mathrm{sVCAM}-1$ & 341 \\
\hline
\end{tabular}

M, male; F, female; $\downarrow$, decreased; PAI-1, plasminogen activator inhibitor-1; =, no effect on; CRP, C-reactive protein; MCP-1, monocyte chemoattractant protein-1; sICAM-1, soluble intercellular adhesion molecule-1; sP-selectin, soluble P-selectin; sVCAM-1, soluble vascular cell adhesion molecule-1; sE-selectin, soluble E-selectin; RANTES, regulated on activation, normal T expressed and secreted; IFN, interferon; sTNFR, soluble TNF receptor; 
WS British Journal of Nutrition

Table 7. Intervention studies investigating the effect of soya intake on markers of low-grade inflammation

\begin{tabular}{|c|c|c|c|c|c|c|}
\hline Subjects & $n$ (sex) & Age (years) & Intake & Study design and duration & Effect on low-grade inflammation & Reference \\
\hline $\begin{array}{l}\text { Hypercholesterolaemic } \\
\text { and postmenopausal }\end{array}$ & $24(F)$ & 55 (SD 5) & $25 \mathrm{~g} / \mathrm{d}$ of soya protein $v$. milk protein & $\begin{array}{l}\text { Randomised, double blind, } \\
\text { controlled; } 6 \text { weeks }\end{array}$ & $\begin{array}{l}=\text { sIL-2R, sE-selectin, sP-selectin, } \\
\text { sICAM-1, sVCAM-1 }\end{array}$ & 351 \\
\hline Healthy postmenopausal & $50(\mathrm{~F})$ & $50-75$ & $40 \mathrm{~g} / \mathrm{d}$ of soya protein $v$. casein & $\begin{array}{l}\text { Randomised, double blind, } \\
\text { controlled; } 3 \text { months }\end{array}$ & $=\mathrm{CRP}$ & 363 \\
\hline Hypercholesterolaemics & $32(\mathrm{M} / \mathrm{F})$ & $57-59$ & $25 \mathrm{~g} / \mathrm{d}$ of soya protein $v$. milk protein & $\begin{array}{l}\text { Randomised, double blind, } \\
\text { cross-over; } 6 \text { weeks }\end{array}$ & $=\mathrm{CRP}, \mathrm{IL}-6$ & 355 \\
\hline Healthy postmenopausal & $55(F)$ & $47-72$ & $\begin{array}{c}40 \mathrm{~g} / \mathrm{d} \text { of soya protein low } \\
\text { or high in isoflavones }\end{array}$ & $\begin{array}{l}\text { Randomised, double blind, } \\
\text { controlled; } 6 \text { weeks }\end{array}$ & $=\mathrm{CRP}$ & 354 \\
\hline Healthy and overweight & $35(\mathrm{M})$ & $20-40$ & $32 \mathrm{~g} / \mathrm{d}$ of soya protein $v$. milk protein & $\begin{array}{l}\text { Randomised, double blind, } \\
\text { controlled; } 57 \mathrm{~d}\end{array}$ & $=\mathrm{CRP}$ & 359 \\
\hline Healthy postmenopausal & $52(F)$ & $50-65$ & $706 \mathrm{ml} / \mathrm{d}$ of soya milk $v$. cows' milk & $\begin{array}{l}\text { Randomised, double blind, } \\
\text { controlled; } 14 \mathrm{~d}\end{array}$ & $=\mathrm{CRP}, \mathrm{TNF}-\alpha$ & 362 \\
\hline $\begin{array}{l}\text { Postmenopausal with the } \\
\text { metabolic syndrome }\end{array}$ & $42(F)$ & & $\begin{array}{l}\text { DASH diet with } 1 \text { serving/d of meat, } \\
\text { or soya protein or roasted soya nuts }\end{array}$ & $\begin{array}{l}\text { Randomised, double blind, } \\
\text { controlled; } 14 \mathrm{~d}\end{array}$ & $\begin{array}{l}\text { Soya protein: } \downarrow \text { CRP, } \\
=\text { sE-selectin } \\
\text { Soya nuts: } \downarrow \text { sE-selectin, } \\
\text { IL-18, TNF- } \alpha, \text { CRP }\end{array}$ & 347 \\
\hline Healthy postmenopausal & $34(F)$ & $47-69$ & $26 \mathrm{~g} / \mathrm{d}$ of soya protein $v$. milk protein & $\begin{array}{l}\text { Randomised, double blind, } \\
\text { cross-over; } 6 \text { weeks }\end{array}$ & $\begin{array}{l}=\text { CRP, sE-selectin, } \\
\text { sVCAM-1, sICAM-1 }\end{array}$ & 353 \\
\hline Hypercholesterolaemics & $28(M / F)$ & $>50$ & $\begin{array}{l}37.5 \mathrm{~g} / \mathrm{d} \text { of protein from whole soyabeans, } \\
\text { soyaflour, soyamilk or animal proteins }\end{array}$ & $\begin{array}{l}\text { Randomised, double blind, } \\
\text { cross-over; } 6 \text { weeks }\end{array}$ & $=\mathrm{CRP}$ & 358 \\
\hline $\begin{array}{l}\text { Normotensive and } \\
\text { hypertensive } \\
\text { postmenopausal }\end{array}$ & $60(F)$ & Mean $\sim 55$ & $\begin{array}{l}25 \mathrm{~g} / \mathrm{d} \text { of soya protein (nuts) } \\
v . \text { non-soya protein }\end{array}$ & $\begin{array}{l}\text { Randomised, double blind, } \\
\text { cross-over; } 8 \text { weeks }\end{array}$ & $\begin{array}{l}\text { Normotensives: = CRP, } \\
\text { IL-6, sICAM-1, sVCAM-1 } \\
\text { Hypertensives: = CRP, } \\
\text { IL-6, sICAM-1, } \downarrow \text { sVCAM-1 }\end{array}$ & 361 \\
\hline Healthy postmenopausal & $75(F)$ & $46-74$ & $20 \mathrm{~g} / \mathrm{d}$ of soya protein $v$. milk protein & $\begin{array}{l}\text { Randomised, double blind, } \\
\text { controlled; } 12 \text { weeks }\end{array}$ & $=\mathrm{TNF}-\alpha, \mathrm{IL}-6, \uparrow$ adiponectin & 352 \\
\hline $\begin{array}{l}\text { Normal weight and obese } \\
\text { pre-menopausal }\end{array}$ & $183(F)$ & Mean 43 & 2 servings/d of soya foods $v$. regular diet & Randomised, controlled; 2 years & $=\mathrm{CRP}, \mathrm{IL}-6$, adiponectin & 357 \\
\hline Healthy postmenopausal & $31(F)$ & Mean 54 & 3 cups/d of soya milk $v$. low-fat cows' milk & $\begin{array}{l}\text { Randomised, single blind, } \\
\text { controlled; } 4 \text { weeks }\end{array}$ & $=\mathrm{TNF}-\alpha, \mathrm{IL}-1 \beta, \mathrm{IL}-6$ & 349,350 \\
\hline Overweight and obese & $20(\mathrm{M} / \mathrm{F})$ & Mean 31 & 3 servings/d of soya milk $v$. cows' milk & $\begin{array}{l}\text { Randomised, double blind, } \\
\text { cross-over; } 28 \mathrm{~d}\end{array}$ & $\begin{array}{l}=\text { CRP, TNF- } \alpha, \text { IL-6, IL-15, } \\
\text { MCP-1, adiponectin }\end{array}$ & 364 \\
\hline $\begin{array}{l}\text { Type } 2 \text { diabetics with } \\
\text { nephropathy }\end{array}$ & $41(\mathrm{M} / \mathrm{F})$ & & Soya protein diet $v$. animal protein diet & Longitudinal randomised; 4 years & $\downarrow$ CRP with soya protein diet & 348 \\
\hline $\begin{array}{l}\text { Obese with the metabolic } \\
\text { syndrome }\end{array}$ & $64(\mathrm{M} / \mathrm{F})$ & Mean 45 & $\begin{array}{l}\text { Walnut- or cashew nut-enriched } \\
\text { diet } v \text {. control diet }\end{array}$ & Randomised, controlled; 8 weeks & $\begin{array}{l}\text { Cashew nuts: } \uparrow \text { plasma glucose, } \\
=\text { lipid profiles, serum } \\
\text { fructosamine, blood pressure, } \\
\text { uric acids, S-hs CRP }\end{array}$ & 360 \\
\hline Healthy hyperlipidaemic & $41(\mathrm{M} / \mathrm{F})$ & Mean 62 & $\begin{array}{l}\text { High- and low-isoflavone soya } \\
\text { diet } v \text {. control diet }\end{array}$ & Randomised, cross-over; 3 months & $\begin{array}{l}\text { Isoflavone: } \downarrow \text { blood lipids, } \\
\text { apolipoprotein B, homocysteine, } \\
\text { systolic blood pressure in men, } \\
\uparrow \text { urinary levels of daidzein, } \\
\text { glycitein, genistein, equol, } \\
\text { O-desmethylangolensin }\end{array}$ & 356 \\
\hline
\end{tabular}

F, female; =, no effect on; sIL-2R, soluble IL-2 receptor; sE-selectin, soluble E-selectin; sP-selectin, soluble P-selectin; sICAM-1, soluble intercellular adhesion molecule-1; sVCAM-1, soluble vascular cell adhesion molecule-1; CRP, C-reactive protein; M, male; $\downarrow$, decreased; $\uparrow$, increased; MCP, monocyte chemoattractant protein; S-hs CRP, serum high-sensitivity C-reactive protein; DASH, dietary approaches to stop hypertension. 
and opposing effects on inflammatory cytokine production in vitro of low- and higher-degree of polymerisation flavanols have been reported ${ }^{(412,413)}$. Because of the focus on in vitro exploration of the effects of cocoa flavanols, there is a need for well-designed human studies, using cocoa properly characterised in terms of flavanol content. In an Italian study of over 10000 people, of whom half were free of any chronic disease, 1317 people reported having eaten any chocolate during the past year; 824 ate chocolate regularly in the form of dark chocolate only ${ }^{(414)}$. Regular consumption of small doses of dark chocolate seemed to decrease inflammation: after adjustment for multiple confounders, dark chocolate consumption was inversely associated with CRP concentration. Serum CRP concentrations were $1.32(1.26-1.39 \mathrm{mg} / \mathrm{l})$ in chocolate non-consumers and $1 \cdot 10(1 \cdot 03-1 \cdot 17 \mathrm{mg} / \mathrm{l})$ in consumers. A J-shaped relationship between dark chocolate consumption and serum CRP was observed; consumers of up to 1 serving $(20 \mathrm{~g}$ ) of dark chocolate every $3 \mathrm{~d}$ had significantly lower serum CRP concentrations than nonconsumers or those consuming more than $20 \mathrm{~g}$ chocolate per d. In a small uncontrolled intervention study, healthy subjects ( $n$ 25) consumed dark chocolate $(36.9 \mathrm{~g} / \mathrm{d}$ ) and a cocoa powder drink (30.95g powder/d) for 6 weeks; there was no change in concentrations of CRP, TNF- $\alpha$, IL-1 $\beta$, IL-6 or soluble P-selectin (sP-selectin) ${ }^{(415)}$. Another small ( $n$ 28), uncontrolled intervention study with dark chocolate for 1 week found a reduction in CRP concentration in women but not in men ${ }^{(416)}$. Most recently, Monagas et al. ${ }^{(417)}$ reported the effect of a 4-week randomised cross-over trial of $40 \mathrm{~g}$ cocoa powder in skimmed milk daily $v$. skimmed milk in forty-two older subjects: serum concentrations of sICAM-1 and sP-selectin were lower after the cocoa powder intervention. Thus, there is some evidence that cocoa and cocoa-rich foods reduce low-grade inflammation.

Alcohol. The regular consumption of alcohol-containing beverages such as wine or beer has been reported to be inversely associated with several markers of low-grade inflammation, in a dose-dependent manner: i.e. moderate daily intake (1-2 drinks/d) was often found to be associated with decreased concentrations of inflammatory markers, although this was not the case in all studies (Table 10) ${ }^{(418-432)}$.

The short-term effect of alcohol intake on markers of lowgrade inflammation has been studied in intervention trials which either determined acute effects during the next hours following ingestion or changes compared with baseline after $2-4$ weeks of daily alcohol consumption (Table 11$)^{(433-448)}$ Although most studies were of small size, findings were quite consistent in that there was little change in low-grade inflammation in the hours following alcohol intake. In one study, a decrease in NF- $\mathrm{KB}$ activation was reported after consuming a fat-rich meal when red wine was also consumed compared with consuming the fat-rich meal ${ }^{(438)}$. In general, findings are fairly similar for trials with wine, beer, vodka or ethanol (see Table 11). After 2-4 weeks of daily intake of alcoholic beverages, changes in low-grade inflammation were noted in five out of eight trials, with decreases in concentrations of CRP, cytokines and soluble adhesion molecules, and an increased concentration of adiponectin (see Table 11).
These effects were seen in trials with white or red wine, or beer. The three 'negative' trials ${ }^{(433-436)}$ used red wine or beer. Some variation of outcome between trials is to be expected, notably because the lifestyle and characteristics of participants will not be identical between trials, in particular with regard to the local diet and the amount of daily physical activity. Nevertheless, when taken together, these studies suggest some beneficial long-term effects of moderate daily consumption of wine or beer on low-grade inflammation. It is not clear whether such effects are to due to the alcohol content of the beverages tested, or to other components, such as phenolic compounds in these fermented beverages.

\section{Process-related compounds: advanced glycation end products and advanced lipoperoxidation end products}

\section{Introduction}

Humans have been using fire to cook food for thousands of years. They used boiling, baking, broiling, roasting, grilling or frying to make food more hygienic, digestible, nutritive and durable, and particularly to improve its flavour, aroma and texture. Despite man's long history of exposure to heated food, the demonstration that the Maillard reaction also occurs and is associated with ageing and age-related conditions, such as diabetic complications, atherosclerosis, Alzheimer's disease and hypertension, has raised the question whether dietary Maillard reaction products (MRP) might represent a risk to human health. A plausible mechanism for adverse health effects of dietary MRP could be their potential promotion of a state of low-grade inflammation. In fact, consumption of a Western diet rich in these products was found to correlate with impaired glucose metabolism, insulin resistance and increased risk of cardiovascular and renal disease associated with the metabolic syndrome and related conditions $^{(449,450)}$. However, although excellent work has been published in the past few years, it is still unresolved whether MRP are causally involved in the aetiology of these conditions ${ }^{(451-453)}$. In particular, whether the potential harm of a Western diet is mainly due to the increased intake of Maillard compounds produced by heating of carbohydrate- and/or lipid-rich food or rather to the excess intake of energy, refined sugars and saturated fats, together with the lower intake of fresh fruits and vegetables, can be debated. Alternatively, other factors generated by thermal treatment of food or the thermal destruction of vitamins, polyphenols and antioxidants during food processing could also contribute to adverse health effects.

\section{Advanced glycation end products and advanced lipoxidation end products}

Advanced glycation end products (AGE) are a family of heterogeneous, partly uncharacterised compounds that encompass both pre-melanoidins and melanoidins. Premelanoidins are not coloured and in general exhibit no fluorescence, whereas melanoidins are yellow to brown and are often fluorescent. The pre-melanoidins include uncoloured 
non-cross-linking AGE such as pyrraline, carboxymethyllysine and carboxyethyllysine ${ }^{(454,455)}$. The melanoidins include all the fluorescent cross-linked AGE formed in the 'non-enzymatic browning reaction', such as pentosidine, and crossline, but also non-fluorescent cross-linked AGE such as glyoxal lysine dimer, methylglyoxal lysine dimer and the alkyl formyl glycosyl pyrroles, formed by the reaction between two sugar molecules with a single lysine residue, and arginine-lysine imidazole, believed to be an intermolecular cross-link. In addition, some compounds that are not AGE in the strict sense are sometimes also considered as AGE. These include the products of the Amadori rearrangement such as fructoselysine (formed with glucose) and the unstable, reactive dicarbonyl intermediates formed by the breakdown of sugars or Amadori intermediates, which may be included in the pre-melanoidins. AGE accumulate in the circulation under several physiological and pathological conditions, including diabetes and related disorders. In addition, circulating AGE accumulate when renal function is impaired ${ }^{(456-459)}$. AGE are formed within the body (endogenous AGE) due to at least three mechanisms ${ }^{(460)}$ :

(1) Enhanced carbohydrate (and/or lipid) substrate availability, such as in hyperglycaemia (and/or hyperlipidaemia), which favours the nucleophilic addition of a carbonyl group from a reducing sugar to a free amino group of a protein (protein glycation) to form a reversible Schiff's base, which then rearranges to the more stable ketoamine or Amadori product and subsequently, through dicarbonyl intermediates such as 3-deoxyglucosones, to the irreversible AGE.

(2) Increased oxidative metabolism, such as in the presence of transition metals and oxidative stress, which cause auto-oxidation of glucose (auto-oxidative glycation) or Amadori products (glycoxidation) via formation of dicarbonyl compounds, such as glyoxal.

(3) Increased non-oxidative metabolism, with accumulation of reducing sugars other than glucose, such as in the case of increased glucose flux through the glycolysis and polyol pathway (intracellular glycation) and/or thiamin deficiency, which result in the formation of the AGE precursor methylglyoxal.

AGE may also be derived from food ${ }^{(454,461)}$ or tobacco ${ }^{(462)}$. AGE are formed during cooking and food processing procedures, and could accumulate in the body after intestinal absorption. It has been suggested from experimental animal models and studies in both healthy and type 2 diabetic subjects that dietary AGE represent a significant source of circulating and tissue AGE, although the absorption of individual AGE from food is largely unknown ${ }^{(463)}$. Little is known about possible tissue deposition of food-derived AGE but in healthy animals, it has been shown that AGE are rapidly and completely excreted ${ }^{(464,465)}$. Finally, accumulation of both endogenous and exogenous AGE may be favoured by reduced kidney clearance as in the case of renal failure ${ }^{(466)}$ and by impaired detoxification caused by the utilisation of cofactors of detoxifying enzymes. 
The so-called advanced lipoxidation end products (ALE) are similar to AGE but, rather than originating from sugars and sugar breakdown products, ALE are derived from lipid oxidation ${ }^{(467)}$. Metal-catalysed oxidation of unsaturated lipids such as PUFA and cholesterol results in the formation of lipid hydroperoxides and oxycholesterols, respectively. Lipid hydroperoxides, in the presence of metal ions or at high temperature, form epoxyhydroperoxides, ketohydroperoxides and cyclic peroxides, which decompose to low-molecular-weight breakdown products such as aldehydes, ketones or alcohols, or condense to polymers. Malondialdehyde and 4-hydroxy-2-nonenal are the main aldehydes generated during lipid peroxidation of n-6 PUFA ${ }^{(468)}$, whereas 4-hydroxy-2-hexanal is the predominant compound derived from the oxidation of $n-3$ PUFA $^{(469)}$. Reactive lipid peroxidation products can form ALE by reacting with amino groups of proteins to generate labile or stable adducts or cross-links in protein, some of which may be coloured or fluorescent. Some reaction products, such as carboxymethyllysine, can originate from both sugars and lipid oxidation products ${ }^{(470)}$, and have been called 'either advanced glycation and lipoxidation end products, ${ }^{\text {(466) }}$.

\section{Advanced glycation end products/advanced lipoxidation end products receptors}

The putative deleterious effects of AGE may be attributed to direct physico-chemical effects such as modification of extracellular matrix proteins, resulting in cross-links, leading to increased vascular stiffness or modification of proteins resulting in altered function, as well as via binding to a variety of cell-surface receptors $^{(471)}$. AGE receptors have a dual function and are involved in both AGE removal and AGE-induced cell activation $^{(140)}$. Cell activation can occur via receptor-mediated generation of ROS through both mitochondrial and cytosolic pathways involving the electron transport chain and NAD(P)H oxidase, respectively ${ }^{(472,473)}$. ROS can trigger proinflammatory signalling pathways causing MAPK-dependent activation of transcription factors such as NF- $\mathrm{KB}^{(474-476)}$ and consequent modulation of the gene expression of several pro-inflammatory cytokines ${ }^{(474,475,477)}$.

Several AGE-binding proteins have been identified, including the $42-45 \mathrm{kD}$ receptor for AGE (RAGE), a member of the Ig superfamily ${ }^{(478)}$, the $60 \mathrm{kD}$ protein a $48-\mathrm{kDa}$ member of the oligosaccharyltransferase complex (OST-48) or AGE-receptor (AGE-R)1, the $90 \mathrm{kD}$ protein $80 \mathrm{~K}-\mathrm{H}$ or $\mathrm{AGE}-\mathrm{R} 2^{(479)}$, and the $32 \mathrm{kD}$ protein galectin-3 or AGE- $3^{(480)}$. This redundancy could imply binding and/or functional specificity among AGE receptors; alternatively, not all these receptors might be relevant for AGE binding in vivo ${ }^{(140,481)}$. At present, RAGE seems to be the only receptor involved in cell activation ${ }^{(140)}$. On the other hand, AGE-R1, AGE-R2 and AGE-R3 seem to behave as an AGE-receptor complex rather than individual receptor molecules and exert a predominant scavenging function ${ }^{(482,483)}$.

In addition to the classical AGE receptors, AGE are cleared also by scavenger receptors, which share with AGE receptors the ability to bind modified lipoproteins such as oxidised $\mathrm{LDL}^{(484)}$. The scavenger receptor family can be broadly classified into eight classes $(\mathrm{A}-\mathrm{H})$, which are expressed on 
Table 10. Observational studies on the association between alcohol intake and markers of low-grade inflammation

\begin{tabular}{|c|c|c|c|c|}
\hline Subjects & $n($ sex $)$ & Age (years) & Association with low-grade inflammation & Reference \\
\hline Community based & $2833(\mathrm{M} / \mathrm{F})$ & Adult & U CRP (lowest for 5-7 drinks/week) & 418 \\
\hline Alcohol abusers and moderate drinkers & $530(\mathrm{M})$ & Adult & $\uparrow \mathrm{CRP}$ with higher intake & 419 \\
\hline Population based & $3697(\mathrm{M} / \mathrm{F})$ & $18-90$ & U CRP & 420 \\
\hline Healthy & $340(\mathrm{~F})$ & & $\begin{array}{l}=\mathrm{CRP} \\
\downarrow \text { IL-6 with higher intake }\end{array}$ & 421 \\
\hline Population based (type 2 diabetics) & $600(F)$ & Mean 64 & $\begin{array}{l}\downarrow \text { CRP, leucocyte count with higher intake } \\
\uparrow \text { Adiponectin }(>9.2 \mathrm{~g} / \mathrm{d})\end{array}$ & 422 \\
\hline Population based & 460 & Adult & $=\mathrm{IL}-6$ & 423 \\
\hline Blood donors & $478(\mathrm{M} / \mathrm{F})$ & $40-68$ & $\begin{array}{l}=\text { IL-6, sICAM-1, albumin } \\
\text { U CRP, SAA }(1-40 \mathrm{~g} / \mathrm{d})\end{array}$ & 424 \\
\hline Population based & $1776(\mathrm{M} / \mathrm{F})$ & $18-88$ & $\begin{array}{l}\text { U CRP, leucocyte count } \\
\text { iU Albumin, transferring }\end{array}$ & 425 \\
\hline Population based & $6739(\mathrm{M} / \mathrm{F})$ & $25-74$ & $\begin{array}{l}\text { U CRP, fibrinogen, leucocyte count (lowest for } 1-40 \mathrm{~g} / \mathrm{d} \text { ) } \\
\text { iU Albumin (highest for } 20-40 \mathrm{~g} / \mathrm{d} \text { ) }\end{array}$ & 426 \\
\hline Population based & $5865(\mathrm{M} / \mathrm{F})$ & $>65$ & $\begin{array}{l}=\text { CRP } \\
\downarrow \text { Leucocyte count, fibrinogen with higher intake } \\
\uparrow \text { Albumin with higher intake }\end{array}$ & 427 \\
\hline Healthy health professionals & $1432(\mathrm{M} / \mathrm{F})$ & & $\begin{array}{l}\uparrow \text { sTNFR1, sTNFR2 }(1-2 \text { drinks/d } v .0 \text { drinks/d) } \\
\downarrow \text { CRP, IL-6 (1-2 drinks/d v. } 0 \text { drinks/d) }\end{array}$ & 428 \\
\hline Type 2 diabetic health professionals & $726(\mathrm{M})$ & $47-82$ & $\begin{array}{l}=\text { CRP, sICAM-1 } \\
\downarrow \text { sTNFR2, fibrinogen, sVCAM-1 } \\
\uparrow \text { Adiponectin }(12.5 \mathrm{~g} / \mathrm{d})\end{array}$ & 429 \\
\hline Population based & $11572(\mathrm{M} / \mathrm{F})$ & $>17$ & $\downarrow$ CRP with higher intake & 430 \\
\hline Healthy & $2574(\mathrm{M} / \mathrm{F})$ & $70-79$ & $\begin{array}{l}=\text { TNF- } \alpha, \text { PAl-1 } \\
\text { U CRP, IL-6 (lowest for } 1-7 \text { drinks/week) }\end{array}$ & 431 \\
\hline Patients from general practices & $3158(\mathrm{M})$ & $60-79$ & $\begin{array}{l}=\text { CRP }(\downarrow \text { for higher intake of wine }) \\
\downarrow \text { Fibrinogen with higher intake }\end{array}$ & 432 \\
\hline
\end{tabular}

M, male; F, female; U, U-shaped relationship; CRP, C-reactive protein; $\uparrow$, increased; =, no effect on; $\downarrow$, decreased; sICAM-1, soluble intercellular adhesion molecule-1; SAA, serum amyloid A; iU, inverse U-shaped relationship; sTNFR, soluble TNF receptor; sVCAM-1, soluble vascular cell adhesion molecule-1; PAI-1, plasminogen activator inhibitor 1.

macrophages but also on other cells, including endothelial, mesangial and vascular smooth muscle cells ${ }^{(485,486)}$. Though the ability of AGE receptors and scavenger receptors to clear pro-inflammatory compounds such as AGE and oxidised LDL from the tissue or circulation would seem initially beneficial, the final net effect is dependent upon the balance between this scavenging function and several other functions of these receptors, including the clearance of other modified self and non-self ligands, downstream pathways with formation of cholesterol-laden cells, the initiation of proinflammatory signalling cascades, and the regulation of cellular lipid influx/efflux and synthesis/degradation ${ }^{(487)}$. However, it should be emphasised that the ligand affinity of AGE proteins to scavenger receptors is dependent on their modification, with mildly modified AGE not showing significant affinity ${ }^{(488)}$.

Among the AGE receptors, RAGE was shown to be implicated in atherogenesis, based on the observations that soluble RAGE, which is able to bind circulating AGE and other RAGE ligands, and therefore to prevent their pro-inflammatory effects, inhibited the development of atherosclerosis in diabetic $\mathrm{apoE}^{-/-}$mice ${ }^{(489)}$, arrested its progression when treatment was started after establishment of lesions ${ }^{(490)}$ and prevented experimental diabetic nephropathy ${ }^{(491)}$, which was conversely accelerated by RAGE overexpression ${ }^{(492)}$. In addition, RAGE ablation prevented diabetic nephropathy ${ }^{(493)}$ and neuropathy ${ }^{(494,495)}$. Thus, the cell-surface RAGE appears to be involved in inducing pro-inflammatory signalling while the soluble form of RAGE appears to prevent this, by seques- tering ligands away from the cell-surface RAGE. Conversely, galectin-3 was proposed to exert a prevailing protective role as indicated by reports that galectin-3 ablation resulted in (1) the acceleration of glomerulopathy induced by diabetes $^{(496)}$, (2) increased AGE levels ${ }^{(497)}$ and (3) accelerated ageing ${ }^{(498)}$. In the vessel wall, galectin-3 was induced in proliferating vascular smooth muscle cells and particularly in foam cells from arteries of experimental animal models of atherosclerosis and human patients with advanced atherosclerotic lesions ${ }^{(499,500)}$, and recent studies have shown that galectin-3 ablation accelerates lipid-induced atherogenesis $^{(501)}$. Also AGE-R1 seems to play a protective role, as indicated by resistance to oxidant and inflammatory injury in mesangial cells overexpressing this receptor ${ }^{(482,502)}$. This was supported by the association of low AGE-R1 expression with susceptibility to diabetic nephropathy in non-obese diabetic mice ${ }^{(503)}$ and the presence of diabetic complications in type 1 diabetic patients ${ }^{(504)}$. Finally, the participation of class A and $\mathrm{B}$ scavenger receptors in the process of atherogenesis has been clearly established by a large body of experimental studies, with scavenger receptor AI (SR-AI) and II and CD36 showing a predominant pro-atherogenic role and scavenger receptor BI (SR-BI) exerting a protective function towards lesion development ${ }^{(487)}$. Thus, the net effect of the interaction of AGE with AGE-binding receptors that can result in either clearance or cell activation would be determined by the relative expression of these different receptors on the respective cells. 
Table 11. Intervention studies investigating the effect of alcohol intake on markers of low-grade inflammation

\begin{tabular}{|c|c|c|c|c|c|c|}
\hline Subjects & $n$ (sex) & Age (years) & Alcohol source and alcohol intake & Study design and duration & Effect on low-grade inflammation & Reference \\
\hline Healthy & $16(\mathrm{M} / \mathrm{F})$ & $22-33$ & $\begin{array}{l}\text { Red wine (two doses: } 12 \text { or } 20 \mathrm{~g} / \mathrm{m}^{2} \\
\text { in men and } 7.2 \text { or } 12 \mathrm{~g} / \mathrm{m}^{2} \text { in women) } \\
\text { or vodka (equivalent to high-dose red wine) }\end{array}$ & Acute; up to $9 \mathrm{~h}$ & $\begin{array}{l}\downarrow N F_{\kappa B} \text { activation after a } \\
\text { fat-enriched meal (only } \\
\text { red wine }- \text { both doses) }\end{array}$ & 438 \\
\hline Healthy & 29 & Adult & Red wine, white wine & Acute; $4 \mathrm{~h}$ & $\begin{array}{l}=\text { sP-selectin, von Willebrand } \\
\text { factor, thrombomodulin } \\
\uparrow \beta \text {-Thromboglobulin }\end{array}$ & 439 \\
\hline Healthy & 16 & Adult & Vodka $(1255.2$ joules $\sim 42 \mathrm{~g}$ ) & Acute; $3 \mathrm{~h}$ & $=\mathrm{NF} \kappa \mathrm{B}$ activation & 440 \\
\hline Postmenopausal women & $20(F)$ & & Alcohol (15 g) & Acute; up to $6 \mathrm{~h}$ & $\begin{array}{l}\downarrow \text { CRP (after a high-carbohydrate, } \\
\text { high-fat meal) }\end{array}$ & 442 \\
\hline $\begin{array}{l}\text { Patients with coronary } \\
\text { artery disease }\end{array}$ & $13(\mathrm{M})$ & & White and red wine ( $2-3$ glasses) & $\begin{array}{l}\text { Randomised, controlled, } \\
\text { cross-over, acute; } 6 \mathrm{~h}\end{array}$ & $\begin{array}{l}=\text { sICAM-1, sVCAM-1 } \\
\uparrow \mathrm{IL}-6\end{array}$ & 448 \\
\hline Healthy & $6(\mathrm{M})$ & & Red wine $(60 \mathrm{~g})$ & Acute $24 \mathrm{~h}$ & $=\mathrm{TNF}-\alpha, \mathrm{IL}-2, \mathrm{IL}-4$ & 435 \\
\hline $\begin{array}{l}\text { Chronic pancreatitis } \\
\text { patients and healthy } \\
\text { controls }\end{array}$ & $23(\mathrm{M})$ & Adult & Alcohol $(40 \mathrm{~g})$ & Acute; $24 \mathrm{~h}$ & $\begin{array}{l}=\text { TGF- } \beta \\
\uparrow \text { IL- } 6 \text { in patients } \\
\downarrow \text { MCP- } 1 \text { in patients }\end{array}$ & 443 \\
\hline Healthy & $5(\mathrm{M})$ & $30-37$ & Red wine $(60 \mathrm{~g})$ & Acute; $36 \mathrm{~h}$ & $\begin{array}{l}=\mathrm{IL}-6, \mathrm{IL}-10, \mathrm{IL}-12 \\
\uparrow \mathrm{IL}-8\end{array}$ & 441 \\
\hline Lean and overweight & $20(\mathrm{M})$ & $18-25$ & Beer $(40 \mathrm{~g} / \mathrm{d})$ & 3 weeks & $\begin{array}{l}=\text { Resistin, leptin } \\
\uparrow \text { Adiponectin, grehlin }\end{array}$ & 433 \\
\hline Lean and overweight & $20(\mathrm{M})$ & & Beer $(40 \mathrm{~g} / \mathrm{d})$ & $\begin{array}{l}\text { Randomised, controlled, } \\
\text { cross-over; } 3 \text { weeks }\end{array}$ & $=\mathrm{CRP}$ & 434 \\
\hline $\begin{array}{l}\text { Subjects with increased } \\
\text { waist circumference }\end{array}$ & $34(\mathrm{M})$ & $35-70$ & Red wine $(40 \mathrm{~g} / \mathrm{d})$ & $\begin{array}{l}\text { Randomised, controlled, } \\
\text { cross-over; } 4 \text { weeks }\end{array}$ & $\begin{array}{l}=\text { Resistin } \\
\uparrow \text { Adiponectin }\end{array}$ & 437 \\
\hline Healthy & $87(M / F)$ & Adult & Red wine $(15 \mathrm{~g} / \mathrm{d})$ & $\begin{array}{l}\text { Randomised, controlled, } \\
\text { cross-over; } 3 \text { weeks }\end{array}$ & $\begin{array}{l}=\text { CRP } \\
\downarrow \text { Fibrinogen }\end{array}$ & 444 \\
\hline Healthy & $35(F)$ & Adult & White or red wine $(20 \mathrm{~g} / \mathrm{d})$ & Randomised, cross-over; 4 weeks & $\begin{array}{l}\downarrow \text { CRP, sICAM-1, SCD40L, IL-6, } \\
\text { monocyte adhesion capacity } \\
\text { (both), sVCAM-1 (only red wine) }\end{array}$ & 445 \\
\hline Healthy & $20(\mathrm{M} / \mathrm{F})$ & $45-64$ & Beer $(40 \mathrm{~g} / \mathrm{d}$ for male, $30 \mathrm{~g} / \mathrm{d}$ for female) & $\begin{array}{l}\text { Randomised, diet-controlled, } \\
\text { cross-over; } 3 \text { weeks }\end{array}$ & $\downarrow \mathrm{CRP}$, fibrinogen & 446 \\
\hline Healthy & $20(M)$ & Adult & Sparkling wine or gin $(30 \mathrm{~g} / \mathrm{d})$ & Randomised, cross-over; 4 weeks & $\begin{array}{l}\downarrow \text { CRP, sICAM-1, IL-6, MCP-1 } \\
\text { (only sparkling wine), sVCAM-1, } \\
\text { sE-selectin, P-selectin (both) }\end{array}$ & 447 \\
\hline Healthy & $24(\mathrm{M})$ & Adult & Red wine $(60 \mathrm{~g} / \mathrm{d})$ & 2 weeks & $=\mathrm{TNF}-\alpha, \mathrm{IL}-2, \mathrm{IL}-4, \mathrm{TGF}-\beta$ & 436 \\
\hline
\end{tabular}

M, male; F, female; $\downarrow$, decreased; =, no effect on; sP-selectin, soluble P-selectin; $\uparrow$, increased; CRP, C-reactive protein; sICAM-1, soluble intercellular adhesion molecule-1; sVCAM-1, soluble vascular cell adhesion molecule-1; TGF, transforming growth factor; MCP-1, monocyte chemoattractant protein-1; SCD40L, SCD40 ligand. 
Significance of dietary advanced glycation end products/ advanced lipoxidation end products

The nutritional properties of heated food were initially questioned because of the reduced availability of lysine due to the formation of Amadori-modified lysine ${ }^{(505)}$. However, cooking was also shown to increase the bioavailability of some nutrients, especially proteins derived from some plant sources $^{(451)}$. Subsequently, the concern was raised that AGE (and ALE) formed during the Maillard reaction might exert adverse health effects in cells, tissues and organs. As a potential mechanism, a role of AGE/ALE binding to RAGE followed by triggering of RAGE-dependent downstream signalling events leading to transcription of pro-inflammatory genes has been postulated ${ }^{(460,506)}$. This view is supported by a number of studies conducted in both experimental animal models and human subjects, showing that ingestion of diets containing high AGE levels results in increased levels of circulating AGE (see below). In addition, these high-AGE diets, generated by more severe thermal treatment than the control diets, also resulted in a series of metabolic and micro/macrovascular abnormalities related to diabetes and its long-term complications (see below). However, despite this body of experimental data, a cause-effect relationship between dietary AGE and development and progression of disease conditions related to Western-type lifestyle and dietary habits has not yet been conclusively demonstrated, and a number of issues remain to be solved.

\section{Evidence supporting a potential harmful effect of dietary} advanced glycation end products/advanced lipoxidation end products

Animal studies. In spontaneously diabetic $d b / d b$ mice ${ }^{(507)}$ and in normal mice fed high-fat diets ${ }^{(508)}$, feeding of a low-AGE diet reduced serum AGE levels and improved insulin sensitivity, compared with a high-AGE diet. In addition, in non-obese diabetic mice, a fetal or neonatal low-AGE environment prevented autoimmune diabetes, possibly by reducing antigenic stimulus for T-cell-mediated injury or by attenuating $\beta$-cell damage ${ }^{(509)}$. In both type 1 and 2 diabetes models (i.e. non-obese diabetic and $d b / d b$ mice, respectively) low dietary AGE content provided sustained protection towards the development of diabetic nephropathy ${ }^{(510)}$. Likewise, in genetically hypercholesterolaemic $\mathrm{apoE}^{-/-}$mice, low AGE content in the diet attenuated the development of atherosclerosis after induction of diabetes with streptozotocin ${ }^{(511)}$ and neointimal formation after arterial injury ${ }^{(512)}$. Finally, in $d b / d b$ mice, wound healing was accelerated by a low-AGE diet, with predominant wound contraction, compared with animals on a high-AGE diet, which favoured the epithelialisation mode of wound closure ${ }^{(513)}$.

Human studies. In healthy younger (aged $<45$ years) and older (aged $>60$ years) human subjects, dietary AGE, but not energy intake, correlated with serum AGE levels and markers of oxidative stress (8-isoprostanes), which in turn correlated with serum CRP concentration and insulin resistance ${ }^{(514-521)}$ (Table 12). It is noteworthy that in this study, some healthy individuals showed AGE levels of similar magnitude as found in diabetic patients.

AGE-rich diets used for intervention studies in human subjects are usually generated by more severe thermal treatment of food, particularly by frying, broiling and baking, whereas AGE-poor food is prepared by steaming or simply without heating at all. Therefore, unless specified, these are the methods that were used for the preparation of test and control diets in the studies reviewed below. Taken together, these studies show that dietary AGE content correlates with circulating levels of AGE and with markers of inflammation, oxidative stress, endothelial dysfunction and renal function. One caveat that needs attention for future human studies is the significant contribution of smoking to the endogenous AGE load ${ }^{(462,522)}$.

Diabetic subjects with and without nephropathy were given a single meal of egg-white, cooked with or without fructose, with the AGE-rich food containing about three times more AGE than a single meal of a regular diet ${ }^{(456)}$. There was a significant correlation between dietary AGE content and serum AGE levels. Moreover, the serum AGE levels were directly related to the degree of renal dysfunction, as estimated by the increase in albuminuria and the decrease in creatinine clearance, and renal AGE excretion was inversely correlated with the degree of renal dysfunction. The relationship between dietary AGE intake, serum AGE levels and renal AGE clearance was confirmed in a cross-sectional study performed in long-term haemodialysis and peritoneal dialysis patients with or without diabetes. This study showed that circulating AGE correlated significantly with the AGE content of a regular diet, as estimated by means of $3 \mathrm{~d}$ dietary records and food questionnaires, as well as with blood urea $\mathrm{N}$ level $^{(459)}$. Likewise, in non-diabetic patients on maintenance peritoneal dialysis randomly assigned to two groups consuming either a high- or low-AGE diet for 4 weeks, serum AGE levels correlated with AGE consumption as well as with blood urea $\mathrm{N}$, serum creatinine, total protein, albumin and $\mathrm{P}$, with AGE restriction profoundly reducing circulating $\mathrm{AGE}^{(523)}$. Finally, cross-sectional studies of healthy subjects and of patients undergoing haemodialysis showed a positive relationship between dietary AGE intake and plasma inflammatory markers ${ }^{(515,520)}$.

A number of acute studies examining the effect of a single high-AGE meal on inflammatory markers have been conducted (see Table 12). Although these have been conducted in a variety of subject/patient groups, they show rather similar effects: typically, there is an elevation of various inflammatory markers (CRP, cytokines and soluble adhesion molecules) in the hours after consumption of a high-AGE meal, and this increase does not occur with a low-AGE meal (see Table 12). Furthermore, healthy and diabetic volunteers receiving a single dose of a high-AGE (caffeine-free) cola drink showed an increase in serum PAI-1 ${ }^{(519)}$

In patients on haemodialysis or peritoneal dialysis randomly assigned to a 4-week high- or low-AGE diet, circulating levels of AGE as well as markers of inflammation and endothelial dysfunction (which were markedly elevated at baseline, although not correlated with AGE) decreased significantly in response to $\mathrm{AGE}$ restriction, i.e. the low-AGE $\operatorname{diet}^{(515)}$ 
The findings of this latter study are in accordance with earlier data in diabetic subjects with normal renal function who showed a decrease in several inflammatory markers following 2 or 6 weeks of a low-AGE $\operatorname{diet}^{(521)}$.

\section{Arguments against potentially harmful effects of dietary advanced glycation end products/advanced lipoxidation end products}

In contrast to the aforementioned reports, circulating AGE levels could not be identified as an independent risk factor for cardiovascular or renal outcomes in diabetic subjects with diabetic nephropathy ${ }^{(524)}$, and circulating AGE levels were even inversely correlated with mortality in haemodialysis patients, the latter most probably due to a better nutritional status ${ }^{(525)}$. In addition, a recent study in obese children found lower circulating AGE levels compared with lean controls while inflammatory markers were significantly elevated in obese children ${ }^{(517)}$. Several issues concerning the methodology and interpretation of published data have been raised that may question the alleged adverse health effects of dietary $\mathrm{AGE} / \mathrm{ALE}^{(451,526,527)}$.

A first issue is the large heterogeneity of AGE/ALE and their precursors, which would require examination of the effect of each of these compounds separately. In particular, the bioavailability and metabolic fate of individual AGE/ALE structures should be evaluated to assess whether and to what extent they are digested, absorbed and cleared by the body, and accumulate in tissues to produce any significant adverse health effect ${ }^{(451,527)}$. AGE, which are absorbed as free or short peptide-bound AGE, may be cleared more rapidly and efficiently by the kidney, except when renal function is significantly impaired, than those formed endogenously, which are usually protein-bound. Studies in experimental animals fed or injected with radiolabelled protein-bound AGE and human volunteers receiving diets enriched with MRP or specific AGE have shown that absorption of ingested MRP is variable and dependent on the $\mathrm{MRP}^{(464,465,527-529)}$. Highly cross-linked AGE and melanoidins were predominantly excreted in the faeces while absorbed MRP were in general rapidly excreted by the kidney, with limited accumulation in tissues and organs ${ }^{(465,528-531)}$. Both pyrraline and pentosidine, the latter only in free form, were readily absorbed and excreted in the urine ${ }^{(532,533)}$. There are also reports that some MRP may be degraded by the colonic microflora ${ }^{(534)}$.

Concerning the structural requirements for AGE to interact with their receptor, there is evidence that RAGE does not bind small molecules and simple protein modifications ${ }^{(478)}$. Penfold et al. ${ }^{(535)}$ recently showed that only serum fractions isolated from diabetic patients with nephropathy containing high-molecular-weight proteins $(>50 \mathrm{kD})$ were able to stimulate RAGE but not medium- or low-molecular-weight serum fractions, in line with mechanistic studies that showed no binding of carboxymethyllysine-modified proteins to $\mathrm{RAGE}^{(536-538)}$, and that supramolecular complexes are a prerequisite for RAGE binding ${ }^{(539-542)}$. Together this suggests that dietary AGE, even if they are absorbed from the gut, would have little effect on inflammatory processes via RAGE activation in target tissues, since it appears highly unlikely that free AGE-modified amino acids would be incorporated into nascent proteins. In addition, a few reports show that binding of AGE to RAGE is not sufficient to stimulate inflammatory signalling ${ }^{(538,542,543)}$, suggesting that endotoxin contaminations of AGE preparations used in the in vitro cellular test systems may be responsible for the observed pro-inflammatory effects.

A further issue, which could complicate the interpretation of results, is the suitability of experimental design/models used in animal studies ${ }^{(451)}$. In fact, the amount of AGE/ALE administered, and particularly the extent of their modification, might not correspond to that of AGE/ALE ingested with usual diets, due to the higher extent of modification introduced by the heating procedure used for preparing test diets in published studies ${ }^{(527)}$. In addition, the true AGE content of many test diets has either not been determined or has been questioned by others, as in the case of the ten times concentrated caffeine-free diet cola beverage described above. In fact, others have found low to undetectable AGE levels in unconcentrated normal or diet cola ${ }^{(466,544)}$. Similarly, the food AGE content published by Goldberg et al. ${ }^{(461)}$ and recently updated ${ }^{(545)}$ using a non-validated ELISA method to determine AGE content is difficult to bring in line with the Maillard reaction in general and with other data on food AGE content ${ }^{(451,527,546)}$.

To attribute adverse health effects to any food ingredient, it is essential that this food ingredient be tested independently, e.g. in pure form and possibly out of the food context. Unfortunately, for most published intervention studies of AGE and/or ALE, this has not been the case. The adverse health effects observed with differentially processed/heated food that result in different AGE/ALE contents have been ascribed to these protein modifications, regardless of the altered food context generated by the differential processing/heating. It may also be possible that AGE/ALE formed under these conditions might be markers of other process/ heating-related alterations of food and not themselves the cause for adverse health effects. This is highlighted by the beneficial effects of melanoidins formed during heat processing of food ${ }^{(547)}$. One of the very few studies that investigated the direct effect of pure AGE by adding an AGE-modified protein $v$. a non-modified protein to a basal animal diet and fed to rats for 11 weeks was in fact unable to demonstrate any adverse health effects of the AGE-supplemented diet ${ }^{(548)}$.

A major issue is the difficulty to attribute the adverse health effects observed with thermally modified diets to their AGE/ALE content, since the possibility cannot be ruled out that other products formed such as heterocyclic amines or acrylamide $^{(453)}$ or ingredients destroyed/consumed such as natural antioxidants contained in fruits and vegetables ${ }^{(526)}$ during cooking and food processing may be responsible for these effects. The notion that AGE may not be the agent causing postprandial inflammation is supported by a report showing that volunteers taking a single dose of either a glucose-modified, AGE-rich or a sorbitol-treated, AGE-poor casein preparation showed an identical postprandial increase in NF- $\mathrm{B}$ activation in peripheral blood mononuclear cells ${ }^{(516)}$. There is a significant body of evidence linking 
Table 12. Studies on the association between advanced glycation end products (AGE) intake and markers of low-grade inflammation

\begin{tabular}{|c|c|c|c|c|c|c|}
\hline Subjects & $n(\operatorname{sex})$ & Age (years) & Intake & $\begin{array}{l}\text { Study design and } \\
\text { duration }\end{array}$ & Effects on low-grade inflammation & Reference \\
\hline $\begin{array}{l}\text { Diabetic and non-diabetic } \\
\text { haemodialysis patients }\end{array}$ & $189(\mathrm{M} / \mathrm{F})$ & $\begin{array}{l}\text { Mean 61(SD 14) } \\
\text { Mean 50(SD 14) }\end{array}$ & Dietary AGE & Cross-sectional & $\begin{array}{l}\uparrow \text { CRP, sVCAM- } 1, \text { TNF- } \alpha \\
=\text { PAI- } 1\end{array}$ & 515 \\
\hline Healthy stratified by age groups & $172(\mathrm{M} / \mathrm{F})$ & $\begin{array}{l}18-45 \\
60-80\end{array}$ & Dietary AGE & Cross-sectional & Correlation of AGE intake with CRP & 520 \\
\hline $\begin{array}{l}\text { Obese type } 2 \text { diabetics, } \\
\text { smokers and non-smokers }\end{array}$ & 13 & Mean 57(sD 10) & High-AGE meal & Acute; up to $4 \mathrm{~h}$ & $\begin{array}{l}\uparrow \text { CRP, sICAM-1, sVCAM-1, sE-selectin } \\
=\text { TNF- } \alpha, \text { IL-6, IL-8, fibrinogen }\end{array}$ & 518 \\
\hline Healthy and type 2 diabetics & $\begin{array}{l}10 \text { Healthy }+44 \\
\text { type } 2 \text { diabetics }(M / F)\end{array}$ & $\begin{array}{l}\text { Mean 43(SD 13) (Healthy) } \\
\text { Mean 51(SD 13) (Diabetics) }\end{array}$ & High-AGE cola drink & Acute; $90 \mathrm{~min}$ & $\begin{array}{l}\uparrow \text { PAI-1 } \\
=\text { sICAM-1 }\end{array}$ & 519 \\
\hline Healthy, non-smokers & 9 & Mean 32(SD 8) & High- and low-AGE meal & Acute; $2 \mathrm{~h}$ & $\begin{array}{l}\uparrow \text { NF-кB activation in both groups } \\
\text { (effect of meal not of AGE?) }\end{array}$ & 516 \\
\hline $\begin{array}{l}\text { Overweight type } 2 \text { diabetics, } \\
\text { smokers and non-smokers }\end{array}$ & $20(\mathrm{M} / \mathrm{F})$ & Mean 55(SD 10) & High- and low-AGE meal & $\begin{array}{l}\text { Cross-over, } \\
\text { acute; up to } 6 \mathrm{~h}\end{array}$ & $\begin{array}{l}\text { High-AGE meal: } \\
\uparrow \text { IL-6, sICAM-1, sVCAM-1, sE-selectin } \\
=\text { CRP, TNF- } \alpha \text {, fibrinogen } \\
\text { Low-AGE meal: } \\
\downarrow \text { IL-6, sICAM-1, sVCAM-1 } \\
=\text { CRP, TNF- } \alpha \text {, sE-selectin, fibrinogen }\end{array}$ & 514 \\
\hline $\begin{array}{l}\text { Non-smoking overweight } \\
\text { diabetics }\end{array}$ & $11(\mathrm{M} / \mathrm{F})$ & Mean 52(sD 17) & High- and low-AGE diet & Cross-over; 2 weeks & $\begin{array}{l}\downarrow \text { TNF- } \alpha \text { mRNA, sVCAM-1 } \\
\text { with low-AGE diet } \\
=\text { CRP }\end{array}$ & 521 \\
\hline $\begin{array}{l}\text { Non-smoking overweight } \\
\text { diabetics }\end{array}$ & $13(\mathrm{M} / \mathrm{F})$ & $\sim 62$ & High- and low-AGE diet & $\begin{array}{l}\text { Randomised, } \\
\text { parallel; } 6 \text { weeks }\end{array}$ & $\begin{array}{l}\uparrow \text { CRP with high-AGE diet } \\
\text { and } \downarrow \text { low-AGE diet } \\
\uparrow \text { TNF- } \alpha \text { with high-AGE diet } \\
\downarrow \text { sVCAM-1 with low-AGE diet }\end{array}$ & 521 \\
\hline $\begin{array}{l}\text { Renal failure patients on } \\
\text { dialysis }\end{array}$ & $18(\mathrm{M} / \mathrm{F})$ & & High- and low-AGE diet & $\begin{array}{l}\text { Randomised, } \\
\text { parallel; } 4 \text { weeks }\end{array}$ & $\begin{array}{l}\downarrow \text { CRP, TNF- } \alpha, \text { sVCAM-1 and PAI-1 } \\
\text { with low-AGE diet }\end{array}$ & 515 \\
\hline $\begin{array}{l}\text { Healthy obese children } \\
v . \text { lean controls }\end{array}$ & $\begin{array}{l}18(11 \mathrm{M} / 7 \mathrm{~F}) \\
18(8 \mathrm{M} / 10 \mathrm{~F})\end{array}$ & $\begin{array}{l}5-18 \\
4-17\end{array}$ & - & Cross-sectional & $\begin{array}{l}\text { Obese had lower plasma AGE and } \\
\text { higher CRP and IL-6 }\end{array}$ & 517 \\
\hline
\end{tabular}

M, male; F, female; $\uparrow$, increased; CRP, C-reactive protein; sVCAM-1, soluble vascular cell adhesion molecule-1; =, no effect on; PAl-1, plasminogen activator inhibitor-1; sICAM-1, soluble intercellular adhesion molecule-1; $\downarrow$, decreased. 
food intake with postprandial inflammatory signalling. In fact, postprandial hyperglycaemia has been shown to be associated with increased NF- $\mathrm{B}$ activation in patients with type 2 diabetes ${ }^{(549)}$. Moreover, an oral glucose load, or better the mean glucose excursions following an oral glucose load, were associated with urinary excretion of 8-iso PGF2 $\alpha$ as a marker of oxidative stress within $2-3 \mathrm{~h}$ after the challenge ${ }^{(550)}$, and oral glucose and/or high fat additively increased inflammatory markers (CRP and IL-6) and oxidative stress (nitrotyrosine), while reducing flow-mediated dilatation $^{(167,551)}$ in diabetic subjects ${ }^{(552)}$. This activation during postprandial hyperglycaemia or hyperlipidaemia may be due to acute changes in markers of glycoxidative and lipoxidative stress and may partly underlie the association of postprandial derangements and cardiovascular risk ${ }^{(553)}$. These observations have prompted the hypothesis that it is the sugar and lipid content of meals which is related to postprandial inflammation, oxidative stress and vascular dysfunction $^{(157,167,551,554)}$. In addition, AGE precursors and AGE/ALE might simply be markers of oxidative processes and inflammation, which would be the true injurious mechanism ${ }^{(455,555,556)}$. The reported beneficial effect of agents such as aminoguanidine ${ }^{(557)}$ in preventing MRP accumulation following ingestion of high-AGE diets could be attributed to the metal-chelating and antioxidant properties of this drug $^{(455)}$. The increased circulating and tissue AGE levels induced by AGE-enriched meals might result from oxidant stress-dependent endogenous AGE formation. The reported attenuation of inflammatory changes induced by fatty meals by treatment with antioxidants is in keeping with this view $^{(456,514)}$. Likewise, the preventive effect of benfotiamine, a transketolase activator that directs glucose substrates to the pentose phosphate pathway, might depend on the consequent blockade of endogenous dicarbonyls and AGE formation as well as of other hyperglycaemia-induced pathways ${ }^{(536)}$. In addition, benfotiamine administration restores normal thiamin levels, which have been reported to be reduced in diabetic patients ${ }^{(538)}$, and compensate for decreased thiamin ingestion with food subjected to severe thermal treatment, which is known to degrade this heat-labile vitamin ${ }^{(537,558,559)}$.

Acute $v$. chronic exposure to AGE-enriched food could produce different effects, and, under both experimental conditions, post-meal values should be considered separately from basal fasting values. Also, the use of animals such as rodents, which are not accustomed to the intake of heated food, might result in outcomes that cannot be directly applied to humans. In addition, the effects of AGE/ALE-rich diets may be different in healthy subjects and patients with diabetes, other age-related disorders or renal insufficiency. The large variation in serum AGE levels detected in younger and older healthy subjects ${ }^{(520)}$, with some individuals showing values similar to those found in diabetic patients, would suggest that AGE might not be harmful unless a concomitant disease condition is present.

The argument that highly cooked or processed food may be responsible for adverse health effects because of their elevated AGE content may be countered by the findings that vegetarians consistently present with higher circulating AGE levels than individuals eating a Western diet ${ }^{(560)}$. Also, the recent report that obesity in children, while correlating with increased oxidative stress and inflammatory markers, showed an inverse correlation with circulating AGE levels is in disagreement with this argument ${ }^{(517)}$.

Another important issue is the reliability of methods for AGE/ALE quantification that are used by different groups ${ }^{(452,527,561)}$. In fact, the use of antisera for quantitative immunoassays of protein-bound AGE is questionable because the specificity of the antibodies is often difficult to ascertain and monospecific antibodies are not commercially available. Furthermore, proteins used to block non-specific binding in immunoassays may also contain AGE epitopes, and thus interact with the detection system. Finally, because of steric constraints, not all AGE epitopes on the protein may be available for interaction with the antibody, and factors competing for the reaction between the anti-AGE antibody and its antigen, including anti-AGE auto-antibodies and, possibly, complement, have been demonstrated in plasma ${ }^{(562,563)}$. Thus, AGE measurements with immunoassays yield only semi-quantitative results and results obtained with immunoassays in both serum samples and food should be interpreted with care. The indirect immunoassay used by Vlassara and co-workers only allows results to be expressed in units, thus making comparison with other analytical data impossible. A much better approach for the quantitative determination of specific AGE epitopes in proteins is the use of specific analytical techniques, such as liquid chromatography-MS-MS, for the analysis of specific AGE in protein hydrolysates ${ }^{(544)}$. Application of this kind of analytical technique could lead to a more comprehensive understanding of the putative effects of AGE formed by the Maillard reaction in food as well as in the body of healthy and diabetic human subjects. In fact, conflicting carboxymethyllysine levels in different foodstuffs have been reported using either an indirect ELISA method ${ }^{(461)}$ or an ultra performance liquid chromatography-MS-MS method ${ }^{(546)}$.

A relevant objection to the hypothesis that dietary AGE/ALE have adverse health effects is the fact that the human organism is equipped with very effective and redundant defence mechanisms limiting the digestion and intestinal absorption of these compounds, which are also trapped in the gastrointestinal tract and detoxified in intestinal epithelia or, for those that are absorbed into the blood or lymph, in liver and other tissues. Based on the pivotal role of the gastrointestinal tract in the protection against ingested MRP, it would be important to assess whether AGE/ALE that are not absorbed have any adverse effects on the intestinal mucosa and/or colonic microflora. Preliminary studies have shown that a high-AGE diet did not affect the number or class of bifidobacteria and sulphate-reducing bacteria, which have beneficial and detrimental effects, respectively ${ }^{(451,564)}$. In addition, Morales et $a{ }^{(547)}$ recently summarised the beneficial health effects of melanoidins generated during heat processing of food.

Despite all these arguments, it might be prudent to advise renal failure patients to decrease their intake of highly heated food. This may not necessarily be due to the presence of AGE/ALE in thermally treated food but rather to other factors present/absent in thermally treated food. 
Finally, there may even be a 'chemoprotective' role of individual dietary AGE/ALE due to antioxidant ${ }^{(565,560)}$ and anti-cancer ${ }^{(567)}$ properties, the former in part attributable to pronyllysine modification of lysine residues of proteins detected in certain foods including bread crust and malt ${ }^{(568)}$.

\section{Research gaps}

Although there appears to be suggestive evidence for a potential role of dietary AGE in inducing low-grade chronic inflammation, insulin resistance and vascular dysfunction, the supporting proof so far has been weak, inconclusive and even contradictory. What the available studies have been able to consistently show is the relationship between dietary AGE/ALE exposure and postprandial circulating AGE levels ${ }^{(520)}$. Whether the uptake of AGE/ALE from the diet is responsible for adverse biological consequences such as chronic low-grade inflammation, vascular dysfunction or insulin resistance will require further investigations.

The following research gaps have been identified:

(1) There is a need for reliable analytical methods for food AGE with inter-laboratory cross-validations.

(2) There is a need for a food AGE database based on reliable analytical methods.

(3) There is a need for preclinical and clinical studies with pure and well-characterised AGE independent of food in both healthy individuals and diabetic patients.

(4) There is a need to discriminate the biological effects induced by protein-bound $v$. free well-characterised AGE.

(5) There is a need for toxicological evaluation of wellcharacterised AGE in animal studies.

(6) There is a need to expand investigation of the (patho)physiological role of food-derived ALE and lipid peroxidation products.

(7) There is a need to evaluate the (patho)physiological consequences of highly heated food beyond their AGE/ALE content.

(8) There is a need to balance potentially adverse health effects of AGE with the potentially beneficial health effects of melanoidins, both of which are generated by thermal treatment of food.

\section{Macronutrients and low-grade inflammation}

\section{Fatty acids}

Dietary fatty acids may affect inflammatory processes through modulation of eicosanoid metabolism, and by eicosanoid-independent mechanisms such as regulation of membrane and cytosolic signalling processes that influence the activity of transcription factors involved in inflammation ${ }^{(569,570)}$. These latter transcription factors include NF-кB and PPAR- $\gamma$, both of which are sensitive to fatty acids. There is an intriguing interaction between these latter two transcription factors because PPAR- $\gamma$ inhibits NF-кB activation $^{(571)}$. A number of different fatty acids including saturated, monounsaturated, trans, conjugated linoleic and polyunsaturated of both $n-6$ and $n-3$ families have been investigated in the context of inflammation. They have been examined in many model systems, typically with isolated cells in culture and in animal models of inflammatory conditions, as well as in studies in human volunteers and in various patient groups. In addition, associations between intake or status of various fatty acids and inflammatory markers have been examined in human studies.

SFA. In vitro studies have suggested that SFA may promote inflammatory processes. Exposure of myotubes or adipocytes to the SFA palmitic acid (16:0) increased IL-6 mRNA expression and subsequent protein production, possibly via activation of $\mathrm{NF}-\kappa \mathrm{B}^{(572,573)}$. Monocytes are activated directly by SFA, especially lauric acid (12:0), via TLR-4 and through this mechanism induce $\mathrm{NF}-\kappa \mathrm{B}$ activity ${ }^{(574,575)}$. A limited number of observational studies have investigated the relationship between SFA exposure and circulating markers of inflammation (Table 13) $)^{(373,374,576-585)}$. Fernandez-Real et $a l .{ }^{(577)}$ did not see any relationship between serum SFA and CRP or IL-6 in lean individuals, while in overweight individuals, serum SFA were positively associated with IL-6 concentration, and the ratio of SFA: $n-6$ or $n-3$ PUFA was positively associated with IL-6 and CRP concentrations, respectively. A study in overweight adolescents showed positive relationships between total SFA in plasma phospholipids or cholesteryl esters and IL-6, but not CRP, concentration ${ }^{(579)}$. Thus, there is general agreement between two studies in overweight subjects that SFA exposure is associated with higher IL-6 concentration. A study in lean individuals does not show this. An intervention study feeding diets rich in stearic acid (18:0) or in lauric, myristic (14:0) and palmitic acids to men aged 25-60 years for 5 weeks showed higher concentrations of CRP, fibrinogen, IL-6 and sE-selectin compared with a diet enriched in oleic acid $(18: 1 n-9)^{(58)}$. There are few other intervention studies chronically increasing SFA intake in human subjects and reporting inflammatory markers.

Trans-fatty acids. Using a subgroup of the Nurses' Health Study, Lopez-Garcia et al. ${ }^{(580)}$ identified significant positive associations between the intake of trans-fatty acids in the diet and the concentrations of all six inflammatory markers assessed, including CRP, IL-6 and three soluble adhesion molecules. In a 5-week intervention in healthy men, a transfatty acid-enriched diet resulted in higher CRP and IL-6 concentrations than diets rich in oleic acid, stearic acid or the combination of lauric, myristic and palmitic acids ${ }^{(586)}$. Furthermore, the concentration of sE-selectin was higher than in all other dietary groups including the stearic acid and lauric + myristic + palmitic groups. Thus, an association study and an intervention study both demonstrate that dietary trans-fatty acids elevate the concentrations of a range of inflammatory markers including CRP, IL-6 and adhesion molecules.

Conjugated linoleic acids. In vitro and animal feeding studies have suggested marked effects of conjugated linoleic acids (CLA) on inflammation ${ }^{(587-589)}$. However, results from human intervention studies using CLA-rich capsules are equivocal (Table 14) ${ }^{(590-596)}$. For example, two studies demonstrate that CLA, especially the trans-10, cis-12 isomer, increases CRP 
concentration, but not the concentrations of cytokines or soluble adhesion molecules ${ }^{(593,594)}$. However, at least four other studies have failed to show an effect of CLA on CRP concentration (see Table 14). Duration of intervention is not likely to be an explanatory factor for differences in the findings, but, since most studies have used mixtures of CLA isomers in different proportions, the precise dose of the more potent isomer (perhaps the trans-10, cis-12 isomer) may be an explanation. The two studies showing increased CRP with CLA provided $2 \cdot 1^{(594)}$ and $2 \cdot 7^{(593)} \mathrm{g} / \mathrm{d}$ of trans-10, cis-12 CLA. The four studies showing no effect of CLA on CRP used between 0.4 and $2.5 \mathrm{~g} / \mathrm{d}$ of this isomer (see Table 14). The question of whether CLA per se, or whether specific CLA isomers, increase low-grade inflammation requires further study.

Linoleic and $\alpha$-linolenic acids. Linoleic (18:2n-6) and $\alpha$-linolenic (18:3n-3) acids constitute the majority (>95\%) of PUFA in most Western diets, with the former usually being present in an excess of approximately 10-fold over the latter. These two fatty acids are the parent $n-6$ and $n-3$ PUFA, respectively. Because of the role of linoleic acid as the precursor of arachidonic acid $(20: 4 n-6)$, which is, in turn, the substrate for the synthesis of pro-inflammatory eicosanoids such as $\mathrm{PGE}_{2}$ and 4-series leukotrienes, it is widely considered that elevated $n-6$ and low $n-3$ fatty acids (i.e. a high $n-6: n-3$ PUFA ratio) in the diet will promote inflammation. However, available evidence does not support this contention.

Dietary intakes of linoleic acid were not associated with CRP, IL-6, sTNFR1 or sTNFR2 concentrations in subgroups of the Physicians' Health Study and the Nurses' Health Study ${ }^{(583)}$. The concentration of linoleic acid in blood lipids ${ }^{(578,579)}$ or granulocytes ${ }^{(374)}$ was not associated with CRP or IL-6 concentration, although a large Swedish study reported an inverse association between linoleic acid in cholesteryl esters and CRP concentration ${ }^{(582)}$. Linoleic acid makes a major contribution to the fatty acids within blood lipids, especially cholesteryl esters, where it is the predominant $n-6$ PUFA. Total $n-6$ PUFA in serum fatty acids in overweight, but not in lean, subjects were inversely associated with IL-6 but not CRP concentration ${ }^{(577)}$. The ratio of SFA: $n-6$ PUFA in serum lipids or in plasma phospholipids was positively associated with IL-6 but not CRP concentration in overweight subjects ${ }^{(577,579)}$. This suggests that decreasing SFA status while increasing n-6 PUFA status might reduce low-grade inflammation. An intervention study in thirty-eight healthy male and female volunteers (mean age 27 years) compared diets with 18\% energy from oleic acid and $4 \%$ from linoleic acid or $4 \%$ energy from oleic acid and $12 \%$ from linoleic acid; plasma sICAM-1 concentrations were not different between these two groups ${ }^{(597)}$, suggesting that exchange of oleic acid for linoleic acid, while keeping SFA intake constant, does not affect this marker of inflammation.

Dietary $\alpha$-linolenic acid intakes were not associated with CRP, IL-6, sTNFR1 or sTNFR2 concentrations in one study on subgroups of the Physicians' Health Study and the Nurses' Health Study ${ }^{(583)}$. In a second study on another subgroup of the Nurses' Health Study, $\alpha$-linolenic acid intakes were not associated with CRP, sICAM-1, sE-selectin or sTNFR2 concentrations but were associated with lower IL-6 and sVCAM-1 concentrations ${ }^{(373)}$. The concentration of $\alpha$-linolenic acid in blood lipids ${ }^{(578,579,582)}$ or granulocytes ${ }^{(374)}$ was not associated with CRP or IL-6 concentration. Data from an Italian study showed no association between $\alpha$-linolenic acid in plasma fatty acids and several cytokines including IL-6 and TNF- $\alpha$, but there was an inverse association with $\mathrm{CRP}^{(578)}$. These association studies suggest a modest anti-inflammatory effect of $\alpha$-linolenic acid.

Several intervention studies have involved high $\alpha$-linolenic acid intakes usually by providing flaxseed oil in capsules or in liquid form or foodstuffs made using flaxseed oil ${ }^{(598)}$. Frequently, these studies have used a control group with a high intake of linoleic acid, with the comparison essentially being replacement of linoleic acid with $\alpha$-linolenic acid. Thus, the focus of these studies is essentially to explore the importance of the $n-6: n-3$ PUFA ratio of the diet. If linoleic and $\alpha$-linolenic acids have similar effects on low-grade inflammation, then studies exchanging one of these fatty acids with the other would be likely to see little effect. Table 15 summarises intervention studies with $\alpha$-linolenic acid that have measured markers of low-grade inflammation as an outcome. Findings are inconsistent with a number of studies identifying the effects of $\alpha$-linolenic acid on some markers and not others, and some studies finding no effects (Table 15) ${ }^{(599-606)}$. Many studies have used very high intakes of $\alpha$-linolenic acid, relative to typical habitual intakes. The study of Paschos et $a{ }^{(601)}$ is enlightening because it showed that $\alpha$-linolenic acid $(8.1 \mathrm{~g} / \mathrm{d}$ for 12 weeks) was less effective (i.e. induced fewer and smaller effects) against a more healthy, than against a less healthy, background diet. Thus, dose, duration, sample size and the nature of the background diet are possible contributors to the varied findings of studies with $\alpha$-linolenic acid. However, what is apparent from these observations is that a substantial increase in the intake of $\alpha$-linolenic acid can decrease low-grade inflammation as indicated by circulating CRP, IL-6 or soluble adhesion molecules (see Table 15). The study of Zhao et al. ${ }^{(606)}$ provides further insight: the change in CRP and sVCAM-1 concentrations was correlated with the change in the concentrations of different serum $n$-3 PUFA $(\alpha$-linolenic acid, EPA, docosapentaenoic acid (22:5n-6) and DHA) that had occurred. The only significant relationships were inverse and between the change in EPA status and the changes in CRP and sVCAM-1 concentrations. This suggests that the anti-inflammatory effect seen with the very high intake of $\alpha$-linolenic acid in this study is due to the conversion of $\alpha$-linolenic acid to EPA. A suitable conclusion may be that high intakes of $\alpha$-linolenic are anti-inflammatory acting via the $\alpha$-linolenic acid derivative, EPA.

Several of the intervention studies with $\alpha$-linolenic acid have involved a group consuming a high intake of linoleic acid, frequently as the control for the high $\alpha$-linolenic acid intake. These groups provide some information about the impact of linoleic acid on low-grade inflammation. The study of Rallidis et al. ${ }^{(603,604)}$ involved a control group consuming about $11 \mathrm{~g} / \mathrm{d}$ of linoleic acid in addition to the dietary intake; this approximately doubled linoleic acid intake. The increase in linoleic acid intake did not alter the concentrations 
WS British Journal of Nutrition

Table 13. Observational studies on the association between fatty acid intake or status and markers of low-grade inflammation

\begin{tabular}{|c|c|c|c|c|c|}
\hline Subjects & $n$ (sex) & Age (years) & Exposure & Association with low-grade inflammation & Reference \\
\hline $\begin{array}{l}\text { Referrals for } \\
\text { coronary angiography }\end{array}$ & $269(\mathrm{M} / \mathrm{F})$ & Mean $\sim 60$ & Granulocyte fatty acids & $\begin{array}{l}\text { Linoleic acid, } \alpha \text { LNA, arachidonic acid, } \\
\text { docosapentaenoic acid: = CRP } \\
\text { EPA: } \downarrow \text { CRP (trend) } \\
\text { DHA: } \downarrow \text { CRP }\end{array}$ & 374 \\
\hline At risk of $\mathrm{CHD}$ & $152(\mathrm{M})$ & Mean 70(SD 3) & $\begin{array}{l}\text { Serum non-esterified } \\
\text { arachidonic acid, } \\
\text { EPA and DHA }\end{array}$ & $\begin{array}{l}\text { Serum non-esterified arachidonic acid: } \\
=\text { sICAM-1, sE-selectin, thrombomodulin, tissue } \\
\text { plasminogen activator antigen } \\
\downarrow \text { sVCAM-1, von Willibrand factor } \\
\text { Serum non-esterified EPA: } \\
=\text { thrombomodulin, tissue plasminogen } \\
\text { activator antigen } \\
\downarrow \text { sVCAM-1, sICAM-1, sE-selectin, } \\
\text { von Willibrand factor } \\
\text { Serum non-esterified DHA: } \\
=\text { sE-selectin, thrombomodulin, tissue } \\
\text { plasminogen activator antigen, von Willibrand factor } \\
\downarrow \text { sVCAM-1, sICAM-1 }\end{array}$ & 585 \\
\hline $\begin{array}{l}\text { Healthy and } \\
\text { overweight }\end{array}$ & $\begin{array}{l}109 \text { Healthy }(\mathrm{M} / \mathrm{F}) \\
\text { and } 123 \\
\text { overweight }(\mathrm{M} / \mathrm{F})\end{array}$ & $\begin{array}{l}\text { Mean } 38 \text { (healthy) } \\
\text { and mean } \\
44 \text { (overweight) }\end{array}$ & Serum fatty acids & $\begin{array}{l}\text { Healthy subjects: } \\
\text { Total SFA, } n \text {-6 PUFA, } n-3 \text { PUFA: = CRP, IL-6 } \\
\text { Overweight subjects: } \\
\text { Total SFA: } \uparrow \text { IL-6, = CRP } \\
\text { Total } n-6 \text { PUFA: = CRP, } \downarrow \text { IL-6 } \\
\text { Total } n-3 \text { PUFA: = IL-6, } \downarrow \text { CRP } \\
\text { Saturated: } n-6 \text { PUFA: } \uparrow \text { IL-6, = CRP } \\
\text { Saturated: } n-3 \text { PUFA: } \uparrow \text { CRP, }=\text { IL-6 }\end{array}$ & 577 \\
\hline Healthy & $405(\mathrm{M})$ and $454(\mathrm{~F})$ & $\begin{array}{l}\text { Mean } \sim 60 \\
\quad(\text { men) and } \\
\sim 42 \text { (women) }\end{array}$ & $\begin{array}{l}\text { Dietary intakes of linoleic } \\
\text { acid, } \alpha \text { LNA, EPA + DHA } \\
\text { as percentage of energy } \\
\text { determined from FFQ }\end{array}$ & $\begin{array}{l}\text { Linoleic acid: = CRP, IL-6, sTNFR1, sTNFR2 } \\
\alpha \text { LNA: = CRP, IL-6, sTNFR1, sTNFR2 } \\
\text { EPA + DHA: = IL-6; } \downarrow \text { CRP, sTNFR1, sTNFR2 }\end{array}$ & 583 \\
\hline Healthy & $727(\mathrm{~F})$ & Mean $\sim 56$ & $\begin{array}{l}\text { Dietary intakes of } \alpha \text { LNA, } \\
\text { EPA }+ \text { DHA, total } n-3 \text { PUFA } \\
\text { as g/d determined from FFQ }\end{array}$ & $\begin{array}{l}\alpha \text { LNA: = CRP, sTNFR2, sICAM-1, } \\
\text { sE-selectin; } \downarrow \text { IL-6, sVCAM-1 } \\
\text { EPA + DHA: = IL-6, sTNFR2; } \downarrow \text { CRP, } \\
\text { sICAM-1, sVCAM-1, sE-selectin } \\
\text { Total } n-3 \text { PUFA: = sTNFR2; } \downarrow \text { CRP, IL-6, } \\
\text { sICAM-1, sVCAM-1, sE-selectin }\end{array}$ & 373 \\
\hline Healthy & $730(\mathrm{~F})$ & Mean $\sim 56$ & $\begin{array}{l}\text { Dietary intake of trans-fatty } \\
\text { acids as } \mathrm{g} / \mathrm{d} \text { determined from } \mathrm{FFQ}\end{array}$ & $\begin{array}{l}\uparrow \text { CRP, IL-6, sTNFR2, sICAM-1, } \\
\text { sVCAM-1, sE-selectin }\end{array}$ & 580 \\
\hline Patients with angina & $291(M / F)$ & Mean $\sim 60$ & $\begin{array}{l}\text { Granulocyte EPA and DHA } \\
\text { Subcutaneous adipose tissue } \\
\text { EPA and DHA }\end{array}$ & $\begin{array}{l}\text { Granulocyte EPA: = sICAM-1, } \\
\text { sVCAM-1, sP-selectin } \\
\text { Granulocyte DHA: = sICAM-1, } \\
\text { sVCAM-1, sP-selectin } \\
\text { Granulocyte total } n \text {-3 PUFA: = sICAM-1, } \\
\text { sVCAM-1, SP-selectin } \\
\text { Adipose EPA: = sICAM-1, } \\
\text { sVCAM-1, sP-selectin } \\
\text { Adipose DHA: } \uparrow \text { sVCAM-1; } \\
\text { = sICAM- } 1 \text {, sP-selectin } \\
\text { Adipose total } n \text {-3 PUFA: } \uparrow \text { sVCAM-1; } \\
\quad=\text { sICAM- } 1 \text {, sP-selectin }\end{array}$ & 576 \\
\hline
\end{tabular}




\begin{tabular}{|c|c|c|c|c|c|}
\hline Subjects & $n(\operatorname{sex})$ & Age (years) & Exposure & Association with low-grade inflammation & Reference \\
\hline Overweight & $60(\mathrm{M} / \mathrm{F})$ & Mean $\sim 12$ & $\begin{array}{l}\text { Plasma phospholipid and } \\
\text { cholesteryl ester fatty acids }\end{array}$ & $\begin{array}{l}\text { Plasma phospholipids: } \\
\text { Total SFA: } \uparrow \text { IL-6; = CRP } \\
\text { Ratio of PUFA to SFA: = CRP; } \downarrow \text { IL-6 } \\
\text { Linoleic acid, } \alpha \text { LNA, EPA, DHA: = CRP, IL-6 } \\
\text { Plasma cholesteryl esters: } \\
\text { Total SFA: } \uparrow \text { IL-6; = CRP } \\
\text { Ratio of PUFA to SFA: = CRP; } \downarrow \text { IL-6 } \\
\text { Linoleic acid, DHA: = CRP, IL-6 } \\
\alpha \text { LNA, EPA: = IL-6; } \downarrow \text { CRP }\end{array}$ & 579 \\
\hline $\begin{array}{l}\text { Japanese general } \\
\text { population } \\
\text { aged }>70 \text { years }\end{array}$ & $971(\mathrm{M} / \mathrm{F})$ & Mean 76(SD 4) & $\begin{array}{l}\text { Dietary intake of } n-3 \text { fatty } \\
\text { acids as g/d determined } \\
\text { from FFQ }\end{array}$ & $\begin{array}{l}\mathrm{EPA}+\mathrm{DHA}: \downarrow \mathrm{CRP} \\
\alpha \mathrm{LNA}:=\mathrm{CRP}\end{array}$ & 581 \\
\hline $\begin{array}{l}\text { General } \\
\text { population } \\
\text { aged }>65 \text { years }\end{array}$ & $1123(\mathrm{M} / \mathrm{F})$ & Mean $\sim 68$ & Plasma fatty acids & 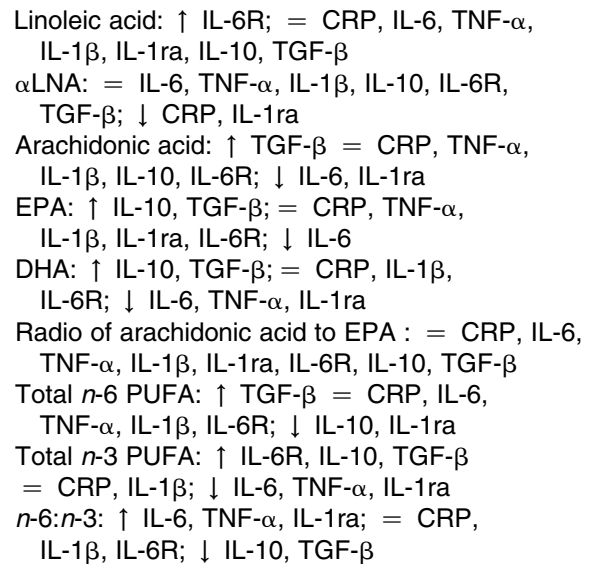 & 578 \\
\hline Healthy & 767 (M) & $\begin{array}{l}50 \text { (fatty acids) } \\
\quad \text { and } 70 \text { (CRP) }\end{array}$ & $\begin{array}{l}\text { Plasma cholesteryl } \\
\text { ester fatty acids } \\
\text { at age } 50 \text { years } \\
v . \text { CRP at age } \\
70 \text { years }\end{array}$ & $\begin{array}{l}\text { Plasma cholesteryl ester } 16: 1 n-7 \text {, } \\
\text { 18:1n-9, } 18: 3 n-6 \uparrow \text { CRP } \\
\text { Plasma cholesteryl ester } 18: 2 n-6 \downarrow \text { CRP }\end{array}$ & 582 \\
\hline Healthy & $511(\mathrm{M} / \mathrm{F})$ & $21-67$ & $\begin{array}{l}\text { Dietary intake of } \\
\text { linoleic acid, } \\
\alpha \text { LNA, EPA and DHA }\end{array}$ & $\begin{array}{l}\text { Linoleic acid: } \downarrow \text { CRP (men only) } \\
\alpha \text { LNA: } \downarrow \text { CRP (men only) } \\
\text { EPA, DHA, EPA + DHA: } \downarrow \text { CRP (weak) } \\
\text { Total } n-6 \text { PUFA: } \downarrow \text { CRP (men only) } \\
\text { Total } n-3 \text { PUFA: } \downarrow \text { CRP (men only) }\end{array}$ & 584 \\
\hline
\end{tabular}

M, male; F, female; $\alpha$ LNA, $\alpha$-linolenic acid; =, no effect on; CRP, C-reactive protein; $\downarrow$, decreased; sICAM-1, soluble intercellular adhesion molecule-1; sVCAM-1, soluble vascular cell adhesion molecule-1; $\uparrow$, increased; STNFR, soluble TNF receptor; sP-selectin, soluble P-selectin; IL-1ra, IL-1 receptor antagonist; TGF, transforming growth factor 
of CRP, IL-6, SAA, sICAM-1 or sE-selectin, but the concentration of sVCAM-1 was decreased ${ }^{(603,604)}$. In the study of Paschos et al. ${ }^{(602)}$, the control group consumed $11.2 \mathrm{~g} / \mathrm{d}$ of linoleic acid on top of their normal intake, which was approximately $11 \mathrm{~g} / \mathrm{d}$. This doubling of linoleic acid intake did not affect TNF- $\alpha$ or adiponectin concentrations. These studies did not alter any aspect of diet but required subjects to consume oil providing linoleic or $\alpha$-linolenic acids on top of the normal diet. Thus, these studies show that markedly increasing linoleic acid intake in those consuming on average about $10 \mathrm{~g} / \mathrm{d}$ does not increase low-grade inflammation.

The study of Zhao et al. ${ }^{(606)}$ included a high linoleic acid intervention group (13\% energy) and the control group consumed what is described as an 'average American diet'. This provided $12.7 \%$ of energy from SFA, $7.7 \%$ from linoleic acid and $0.8 \%$ from $\alpha$-linolenic acid. The corresponding values for the linoleic and $\alpha$-linolenic acid rich diets were 8.5, 12.6 and 3.6 and $8.2,10.5$ and 6.5 , respectively. Thus, the linoleic acid-rich diet contained more $\alpha$-linolenic acid than the control diet, while the $\alpha$-linolenic acid diet contained more linoleic acid than the control diet and almost as much linoleic acid as the linoleic acid diet. Essentially, what this means is that the linoleic acid diet is examining the effect of replacing some SFA with the combination of linoleic and $\alpha$-linolenic acids and that the $\alpha$-linolenic acid diet is examining the effect of replacing some linoleic acid with $\alpha$-linolenic acid. In the linoleic acid group, the concentrations of CRP and sICAM-1 decreased by 45 and $25 \%$, respectively; sVCAM-1 and sE-selectin also fell (by 15 and $7 \%$ ) but these changes were not significant. Thus, replacing about one-third of SFA with linoleic acid plus $\alpha$-linolenic acid decreases low-grade inflammation. This is consistent with the associations described above and confirms that relative to SFA, the combination of linoleic and $\alpha$-linolenic acids is anti-inflammatory. The changes in inflammatory markers seen with the $\alpha$-linolenic acid diet were greater than with the linoleic acid diet ( 75 , $80,37.5$ and $12 \%$ decreases in CRP, sVCAM-1, sICAM- 1 and sE-selectin concentrations, respectively). Since this diet is effectively replacing some linoleic acid with $\alpha$-linolenic acid, relative to the amounts in the linoleic acid diet, these results suggest that $\alpha$-linolenic acid is more potent than linoleic acid with regard to reducing inflammation.

The studies just described have focused on increasing intake of linoleic and $\alpha$-linolenic acids to study their effects. The study of Liou et al. ${ }^{(607)}$ used a different approach. They kept the amount of $\alpha$-linolenic acid in the diet of men aged $20-45$ years constant over the study period at about $1 \%$ of energy. Linoleic acid intake was either 10.5 or $3.8 \%$ of energy. Saturated fat was also constant across the two diets at about $8 \%$ of energy. The manipulation of linoleic acid was at the expense of oleic acid ( 17 or $10 \%$ of energy). The intervention duration was 4 weeks and a random order, cross-over design was used. CRP and IL-6 concentrations were not different after 4 weeks on either diet. This finding is in accordance with that of Turpeinen et al. ${ }^{(597)}$.

Arachidonic acid. Arachidonic acid intake in the diet is low relative to that of its metabolic precursor linoleic acid (approximately $500 \mathrm{mg} / \mathrm{d} v$. approximately $11 \mathrm{~g} / \mathrm{d}$, respectively).
Nevertheless, arachidonic acid is the most prevalent $n-6$ PUFA and PUFA in the membranes of inflammatory cells and other cells that might be involved in low-grade inflammation such as endothelial cells and platelets. This reflects the important functional role of arachidonic acid as a precursor of the eicosanoid family of lipid mediators; this family includes the 2-series PG and the 4-series leukotrienes. Since these eicosanoids are classically associated with inflammatory processes and are targeted by common anti-inflammatory therapies, it is generally considered that arachidonic acid will enhance inflammation. However, observations that classical pro-inflammatory mediators such as $\mathrm{PGE}_{2}$ can also exert anti-inflammatory effects and that arachidonic acid gives rise to anti-inflammatory mediators such as lipoxin $\mathrm{A}_{4}$ have started to challenge the earlier view ${ }^{(608)}$. Several studies have examined the association between arachidonic acid status and markers of low-grade inflammation. There was no association between arachidonic acid in granulocytes and CRP concentration $^{(374)}$. Serum free arachidonic acid was not associated with sICAM-1 or sE-selectin concentrations and was actually inversely associated with sVCAM- 1 concentration ${ }^{(585)}$. Ferrucci et al. ${ }^{(578)}$ reported no association between arachidonic acid in plasma and CRP, TNF- $\alpha$, IL- $1 \beta$, IL-10 and sIL-6R concentrations, while there was an inverse association with IL-6 and IL-1ra concentrations and a positive association with TGF- $\beta$ concentration. These observations suggest either that plasma arachidonic acid has little impact on low-grade inflammation (does not affect CRP or TNF- $\alpha$ ) or that it is anti-inflammatory (lowers IL- 6 ; increases TGF- $\beta$ ).

There are very few intervention studies with arachidonic acid reporting on low-grade inflammation. In an uncontrolled study, Kelley et al. ${ }^{(609)}$ reported higher granulocyte numbers in the blood of a group of ten healthy men (aged $20-38$ years) taking a supplement of $1.5 \mathrm{~g} / \mathrm{d}$ of arachidonic acid for $100 \mathrm{~d}$ compared with numbers after a run-in diet providing $200 \mathrm{mg} / \mathrm{d}$ of arachidonic acid. In another small, but controlled, study, eight subjects aged 55-75 years consumed capsules providing $700 \mathrm{mg} / \mathrm{d}$ of arachidonic acid for 12 weeks ${ }^{(605)}$; there was no effect on plasma sVCAM-1, sICAM-1 or sE-selectin concentrations.

Marine-derived long-chain $n-3$ PUFA. The long-chain $n-3$ PUFA EPA and DHA are found in seafood, especially oily fish. They are also present in fish oils and in certain algal oils; in some preparations, the fatty acids are in a more concentrated form than in natural fish oils. In fish oils, the fatty acids are in the TAG form, but other forms of long-chain $n-3$ PUFA are also available, for example, as phospholipids or ethyl esters. Increased intake of long-chain $n$-3 PUFA results in increased proportions of those fatty acids in inflammatory cell phospholipids ${ }^{(610-614)}$. The incorporation of EPA and DHA into human inflammatory cells is partly at the expense of arachidonic acid, resulting in less substrate available for the synthesis of the classic inflammatory eicosanoids such as $\mathrm{PGE}_{2}$. Through altered eicosanoid production, $n$ - 3 PUFA could affect inflammation and inflammatory processes, although they also exert non-eicosanoid-mediated actions on cell signalling and gene expression. The effects of long-chain $n-3$ PUFA have been examined in many model systems and findings from 
cell-culture systems and from animal models are generally consistent in identifying anti-inflammatory actions ${ }^{(569)}$. Furthermore, clinical trials have demonstrated anti-inflammatory effects and clinical benefit from fish oil administration in diseases with a frank inflammatory basis including rheumatoid $\operatorname{arthritis}^{(615)}$, inflammatory bowel diseases ${ }^{(616)}$ and childhood asthma $^{(617)}$.

Data from subgroups of the Physicians' Health Study and the Nurses' Health Study showed inverse associations between the dietary intake of EPA + DHA and concentrations of CRP, sTNFR1 and sTNFR2 ${ }^{(583)}$, and CRP, sICAM-1, sVCAM-1 and sE-selectin ${ }^{(373)}$. The concentration of either EPA or DHA in granulocyte membranes was inversely associated with CRP concentration in one study ${ }^{(374)}$; the effect of DHA was stronger than that of EPA. Serum non-esterified EPA and DHA were both inversely associated with concentrations of sVCAM-1 and sICAM-1 in patients at risk of $\mathrm{CHD}^{(585)}$; EPA was also inversely associated with sE-selectin concentration. Plasma cholesteryl ester EPA was inversely associated with CRP concentration in overweight subjects ${ }^{(579)}$. In an elderly Italian population, plasma EPA was inversely associated with IL-6 concentration and positively associated with the concentrations of the anti-inflammatory cytokines IL-10 and TGF$\beta^{(578)}$. Furthermore, plasma DHA was inversely associated with IL- 6 and TNF- $\alpha$ concentrations and was also positively associated with the concentrations of IL-10 and TGF- $\beta^{(578)}$. Thus, observational studies suggest that both EPA and DHA are anti-inflammatory.

The ready availability of fish oil capsules has facilitated numerous supplementation studies of long-chain $n-3$ PUFA in various subject groups; these are summarised in Table $16^{(164,605,618-646)}$. Studies have shown that longchain $n$-3 PUFA lower the concentrations of $\mathrm{CRP}^{(621,624,639)}$ IL-6 ${ }^{(621,624,639)}$, TNF- $\alpha^{(639)}$, IL-18 ${ }^{(643)}$, SICAM-1 $^{(625,628,645,646)}$, sVCAM-1 ${ }^{(605,645)}$ and sE-selectin ${ }^{(618)}$ in various subject/patient groups (see Table 16). For example, one study showed an increase in adiponectin concentration when a weight-loss programme and $4.2 \mathrm{~g} / \mathrm{d} \mathrm{EPA}+$ DHA were combined in overweight, insulin-resistant women ${ }^{(632)}$. Thus, there is quite a lot of evidence for anti-inflammatory effects of supplemental long-chain $n$-3 PUFA. In a group of overweight women with type 2 diabetes, 8 weeks of a moderate dose of long-chain $n-3$ PUFA ( $1.8 \mathrm{~g} / \mathrm{d}$ of EPA + DHA) decreased adiposity and reduced expression of a number of inflammation-related genes in the subcutaneous adipose tissue ${ }^{(630)}$. The parallelism between the down-regulation of these genes and the reduction in adiposity and adipocyte diameter by $n-3$ PUFA treatment suggests a positive relationship between adipose cell size and adipose tissue inflammation, agreeing with other observations ${ }^{(31,66)}$. Also these findings in type 2 diabetic women are paralleled by those from rodent studies: 6 weeks of $n$-3 PUFA supplementation prevented adipose tissue inflammation induced by a high-fat diet ${ }^{(647)}$, and the presence of $n$-3 PUFA in the diet of long-term insulin-resistant, sucrosefed rats decreased adipocyte diameter ${ }^{(648)}$ and significantly reduced several inflammation-related genes (unpublished results, Guerre-Millo M, Naour N, Lombardo Y, Clement K, Rizkalla S). These studies suggest that reducing adiposity with $n$-3 PUFA could decrease adipose tissue inflammation and macrophage infiltration. The beneficial effects of $n-3$ PUFA may be linked to local blunting of adipose tissue inflammation.

Despite the large number of positive studies with long-chain $n$-3 PUFA, there are a number of studies that have failed to replicate these findings (see Table 16). Furthermore, two early studies showed an enhancement of selected inflammatory markers following long-chain $n-3$ PUFA administration $^{(629,642)}$. Seljeflot et al. provided $4.8 \mathrm{~g} / \mathrm{d}$ of EPA + DHA in ethyl ester form to hyperlipidaemic male smokers for 6 weeks, while Johansen et al. provided $5 \cdot 1 \mathrm{~g} / \mathrm{d}$ of EPA + DHA in ethyl ester form to patients with CHD for 24 weeks. Both studies identified an increase in sVCAM-1 $(<10 \%)$ and sE-selectin (20\%) concentrations, with no effect on sP-selectin and a decrease in von Willibrand factor and thrombomodulin concentrations. In both cases, the authors ascribed the effect of EPA + DHA on SVCAM-1 and sE-selectin to increased oxidant stress in these subjects.

Thus, although the overwhelming view is that EPA + DHA given at sufficient doses are anti-inflammatory, the evidence from measurements of markers of low-grade inflammation is not entirely consistent. The lack of consistency may be related to differences in: duration of treatment; sample size; characteristics of the populations studied (e.g. age, healthy $v$. diseased, type of disease, smokers $v$. non-smokers); background diet; dose of $\mathrm{EPA}+\mathrm{DHA}$ used; relative contribution of EPA and DHA, since they may have different anti-inflammatory potencies; chemical formulation (e.g. TAG $v$. ethyl ester); degree of oxidative stress present. One other factor that has been recently identified is genetic differences among individuals, which may have an impact on the ability of $n$-3 PUFA to exert an anti-inflammatory effect. This was first identified by Grimble et $a l^{(649)}$ who showed that the ability of fish oil to lower the LPS-stimulated production of TNF- $\alpha$ by blood mononuclear cells was determined in part by polymorphisms within the TNF- $\alpha$ and TNF- $\beta$ genes. Another example of such an interaction was identified by Shen et al. ${ }^{(650)}$. They first identified that the IL-1 $36054 \mathrm{G}>\mathrm{A}$ SNP was significantly associated with CRP and adiponectin concentrations and with the prevalence of the metabolic syndrome among a group of 1120 men and women with a mean age of 49 years. There was also a significant interaction between this polymorphism and erythrocyte membrane $n-3$ PUFA content. Among subjects with low erythrocyte $n-3$ PUFA content (below the median), the $6054 \mathrm{G}$ allele was associated with increased risk of the metabolic syndrome (OR 3.29, 95\% CI $1 \cdot 49,7 \cdot 26$ for GG and OR 1.95, 95\% CI 0.85, $4 \cdot 46$ for GA) compared with the AA genotype, but there were no significant genotype associations among subjects with high erythrocyte $n$-3 PUFA content (above the median). The results suggest that IL-1 $\beta$ genetic variants are associated with measures of chronic low-grade inflammation and the risk of the metabolic syndrome, and that genetic influences were more evident among subjects with low erythrocyte $n$ - 3 PUFA status and so, most probably low $n$-3 PUFA intake. 


\section{Carbohydrates}

Acute postprandial effects of hyperglycaemia. Hyperglycaemia, elevated TAG/NEFA and hyperinsulinaemia are events that are mutually inherent to the initial development of a diabetic state. The effects of the individual events are difficult to study since they always present together. However, some observations have suggested that hyperglycaemia and hypertriacylglycerolaemia/elevated NEFA levels induce independent effects and, when present together, act synergistically to generate oxidative stress, inflammation, impaired endothelial function and vascular disease ${ }^{(651-653)}$. Oxidative stress is known to induce damage to cell membranes, internal cell structures and DNA as well as to induce inflammatory responses (Fig. 8) ${ }^{(654)}$. Ceriello et al. ${ }^{(652)}$ observed that after meals LDL oxidation increases and that this phenomenon is correlated with the degree of hyperglycaemia. This clearly points to hyperglycaemia-induced free radical production that has an impact on a broad range of metabolic events. $\mathrm{NO}$, a potent vasodilator, is assumed to play a key role in this respect. It has been suggested that hyperglycaemia leads to an increased oxidation of $\mathrm{NO}$, thereby reducing NO levels which leads to impairment in vasodilatation ${ }^{(655)}$. Indeed, alloxan diabetic rats are observed to have reduced NO levels in blood ${ }^{(656)}$, and in human subjects, acute hyperglycaemia attenuated endothelium-dependent vasodilation ${ }^{(657)}$. Postprandial hyperglycaemia is also correlated with impaired myocardial perfusion in diabetic patients ${ }^{(658)}$. The fact that such a decrease can be prevented by supplying various antioxidants such as vitamin $\mathrm{C}$, vitamin $\mathrm{E}$ and $\alpha$-lipoic acid ${ }^{(659-662)}$ or L-arginine ${ }^{(656,663)}$, the precursor of $\mathrm{NO}$, suggests that increased NO oxidation and the related NO drain have an impact on cells of the vasculature. This is supported by other evidence. Restoration of NO availability results in normalisation of endothelial function as well as insulin sensitivity ${ }^{(659,660)}$. During an oral glucose tolerance test, plasma antioxidant status, measured as total plasma radical trapping capacity, significantly decreased in normal as well as in diabetic individuals. The consumption of a glycaemic meal increases oxidative stress and reduces antioxidant defences, with the increase being significantly greater with higher levels of hyperglycaemia ${ }^{(169,664,665)}$. Acute postmeal hyperglycaemia was observed to induce the formation of nitrotyrosine, a marker of oxidative stress, in healthy, non-overweight individuals ${ }^{(666)}$. It has been hypothesised that acute hyperglycaemia may induce a drain of vitamin C from cells because vitamin $\mathrm{C}$ and glucose share a common transport system ${ }^{(667)}$ and oxidative stress leads to a use of intracellular vitamin C. Accordingly, Chen et al. ${ }^{(668)}$ provided evidence in vitro that acute hyperglycaemia leads to a significant decrease in leucocyte vitamin C content. Evans et al. ${ }^{(662)}$ developed a unique oxidative stress hypothesis suggesting that chronic elevation of hyperglycaemia (and NEFA) induces an activation of the NF- $\mathrm{kB}, \mathrm{p} 38$ MAPK and $\mathrm{NH}_{2}$-terminal Jun kinase/stress-activated protein kinase pathways. This happens along with the activation of the RAGE, protein kinase $C$ and sorbitol stress pathways. The authors suggest that these events play a key role in causing late complications 


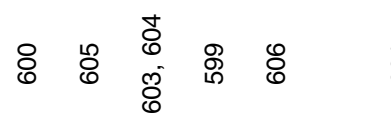

\section{(1)}
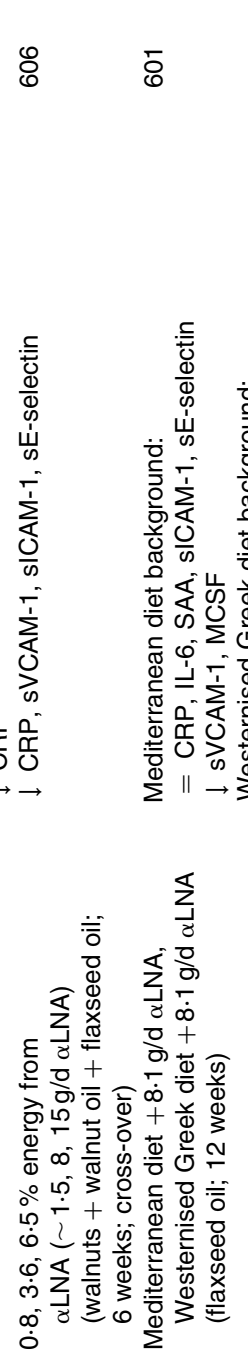

in type 1 and type 2 diabetes, going along with insulin resistance and impaired insulin secretion in type 2 diabetes. Evans et $a l^{(662)}$ provided evidence that elevated glucose causes oxidative stress due to increased production of mitochondrial ROS, non-enzymatic glycation of proteins and glucose auto-oxidation. They also pointed out that elevated NEFA can cause oxidative stress due to increased mitochondrial uncoupling and $\beta$-oxidation, the latter leading to an increased production of ROS and to an activation of stress-sensitive signalling pathways, which in turn impair insulin secretion and action. Thus, oxidative stress induced by acute and chronic elevations in glucose and NEFA plays a key role in causing insulin resistance and $\beta$-cell dysfunction. A range of studies has shown that acute and lasting hyperglycaemia leads to an elevation in dicarbonyls and protein glycation (measured as fructosamine or HbA1c). Glycosylated proteins, as well as their metabolites, AGE, which bind to the AGE receptor RAGE, induce a cascade of signals that initiate an inflammatory response ${ }^{(669)}$. Accordingly, pro-inflammatory transcription factors, inflammatory markers including CRP, IL-6, IL-8, TNF- $\alpha$ and matrix metalloproteinase and markers of endothelial dysfunction VCAM-1, ICAM-1 and E-selectin have been observed to be elevated in response to acute and persistent hyperglycaemia ${ }^{(474,475,514,521,653,662,670,671)}$.

Glycaemic index, glycaemic load and inflammation. Dietary glycaemic index (GI) and glycaemic load (GL) were both positively associated with plasma CRP concentration in 18137 healthy women aged $>45$ years without diagnosed diabetes $^{(672)}$. In another study in 974 subjects aged $42-87$ years, dietary GI was positively associated with CRP concentration $^{(673)}$. Qi et al ${ }^{(315)}$ examined the associations of dietary GI and GL with plasma adiponectin among 780 diabetic men from the Health Professionals' Follow-Up Study. After adjustment for other factors, dietary GI and GL were both significantly inversely associated with plasma adiponectin concentration in a dose-dependent fashion. Adiponectin levels were $13 \%$ lower in the highest quintile of dietary GI than in the lowest quintile. For dietary GL, adiponectin levels were $18 \%$ lower in the highest quintile than in the lowest. Several intervention studies have examined the impact of dietary GI or GL on markers of chronic low-grade inflammation. In one study, thirty-four healthy overweight adults aged 24-42 years received energy-restricted diets with high or low GL for 6 months ${ }^{(674)}$; more subjects in the low GL group showed a decline in serum CRP concentration than in the high GL group. In another study, Shikany et $a l .{ }^{(675)}$ found no differences in CRP, TNF- $\alpha$, IL-6, fibrinogen, STNFR2 or PAI-1 concentrations when overweight or obese men consumed diets with high or low GI for 4 weeks. Likewise, 11-week interventions of high- or low-GL diets in fifteen overweight subjects had no differential effects on CRP, TNF- $\alpha$, IL-6 or MCP-1 concentrations ${ }^{(676)}$. Thus, evidence from large observational studies is highly suggestive that there is a positive association between GI/GL and low-grade inflammation, but intervention trials do not support this convincingly, perhaps because of the small size of most of the latter trials. 
WS British Journal of Nutrition

Table 16. Intervention studies investigating the effect of marine $n$-3 PUFA intake on markers of low-grade inflammation

\begin{tabular}{|c|c|c|c|c|c|}
\hline Subjects & $n(\operatorname{sex})$ & Age (years) & Intake (source; duration) & Effect on low-grade inflammation & Reference \\
\hline Healthy & $58(\mathrm{M})$ in four groups & $21-87$ (mean 56) & $\begin{array}{l}0,1 \cdot 06,2 \cdot 13,3 \cdot 19 \mathrm{~g} / \mathrm{d} \text { EPA + DHA } \\
\text { (FO capsules; up to } 52 \text { weeks) }\end{array}$ & $=\mathrm{TNF}-\alpha, \mathrm{IL}-1 \beta, \mathrm{IL}-1 \mathrm{ra}$ & 620 \\
\hline Healthy and hyperlipidaemic & $\begin{array}{r}20 \text { Healthy }(M / F) \text { and } 39 \\
\text { hyperlipidaemic }(M / F)\end{array}$ & Mean $\sim 51$ & $0,3.6 \mathrm{~g} / \mathrm{d}$ EPA + DHA (EE capsules; up to 6 weeks) & $\begin{array}{l}=\text { sICAM- } 1, \text { sVCAM- } 1 \\
\downarrow \text { sE-selectin }\end{array}$ & 618 \\
\hline Hyperlipaemic smokers & $\begin{array}{l}41 \text { (M) in four groups (two } \\
\text { received } n-3 \text { PUFA) }\end{array}$ & $41-57$ (mean 48) & $0,4.8 \mathrm{~g} / \mathrm{d}$ EPA + DHA (EE capsules; 6 weeks) & $\begin{array}{l}\text { † sVCAM-1, sE-selectin } \\
=\text { sP-selectin, tissue plasminogen } \\
\text { activator antigen } \\
\downarrow \text { von Willibrand factor, thrombomodulin }\end{array}$ & 642 \\
\hline Patients with $\mathrm{CHD}$ & $54(\mathrm{M} / \mathrm{F})$ in two groups & $43-73($ mean 57$)$ & 0,5.1 g/d EPA + DHA (EE capsules; 24 weeks) & $\begin{array}{l}\uparrow \text { sVCAM- } 1 \text {, Se-selectin } \\
=\text { sP-selectin, tissue plasminogen } \\
\text { activator antigen } \\
\downarrow \text { von Willibrand factor, thrombomodulin }\end{array}$ & 629 \\
\hline Healthy and type 2 diabetics & $\begin{array}{l}21 \text { Healthy }(M) \text { and } \\
29 \text { diabetics }(M)\end{array}$ & Mean $\sim 55$ & 2.0 g/d EPA + DHA (FO capsules; 3 weeks) & $\begin{aligned}= & \text { sICAM-1, sVCAM-1, sE-selectin, } \\
& \text { PAl-1 activity, PAl-1 antigen }\end{aligned}$ & 640 \\
\hline Healthy & $24(M / F)$ in three groups & $55-75$ & $\begin{array}{l}\text { 0, } 0.7 \mathrm{~g} / \mathrm{d} \text { DHA (DHA-rich algal oil capsules; } 12 \text { weeks), } \\
1 \mathrm{~g} / \mathrm{d} \text { EPA + DHA (FO capsules; } 12 \text { weeks) }\end{array}$ & $\begin{array}{l}\text { EPA + DHA: } \\
=\text { sICAM- } 1, \text { sE-selectin } \\
\downarrow \text { sVCAM- } 1 \\
\text { DHA: = sICAM- } 1 \text {, sVCAM- } 1 \text {, sE-selectin }\end{array}$ & 605 \\
\hline Obese & $\begin{array}{l}48(\mathrm{M}) \text { in four groups (two } \\
\text { received } n \text {-3 PUFA) }\end{array}$ & Mean 53(SD 7) & 0, 3.5 g/d EPA + DHA (EE capsules; 6 weeks) & $=\mathrm{CRP}, \mathrm{IL}-6, \mathrm{TNF}-\alpha$ & 623 \\
\hline Elderly at risk of $\mathrm{CHD}$ & $\begin{array}{l}171 \text { (M) in four groups } \\
\quad \text { (two received } n-3 \text { PUFA) }\end{array}$ & Mean 70(SD 3) & 0, $2.4 \mathrm{~g} / \mathrm{d}$ EPA + DHA (FO capsules; 18 months) & $\begin{array}{l}=\text { sICAM-1, sVCAM-1, sE-selectin, } \\
\text { tissue plasminogen activator antigen } \\
\downarrow \text { von Willibrand factor, thrombomodulin }\end{array}$ & 619 \\
\hline $\begin{array}{l}\text { Healthy on hormone } \\
\text { replacement therapy }\end{array}$ & $30(F)$ in three groups & Mean 60 & $\begin{array}{l}0,1 \cdot 09,2 \cdot 18 \mathrm{~g} / \mathrm{d} \text { EPA }+ \text { DHA } \\
\quad(\text { FO capsules; } 5 \text { weeks) }\end{array}$ & $\downarrow$ CRP, IL-6 & 624 \\
\hline Healthy & $60(\mathrm{M} / \mathrm{F})$ in three groups & 21-57 (mean 38) & $\begin{array}{l}0,2 \cdot 0,6 \cdot 6 \mathrm{~g} / \mathrm{d} \text { EPA }+ \text { DHA } \\
\text { (FO capsules; } 12 \text { weeks) }\end{array}$ & $=\mathrm{CRP}$ & 633 \\
\hline Myocardial infarction survivors & $300(\mathrm{M} / \mathrm{F})$ in two groups & 28-87 (mean 65) & $0,3.5 \mathrm{~g} / \mathrm{d}$ EPA + DHA (EE capsules; 12 months) & $=$ sICAM-1, sVCAM-1, sE-selectin & 627 \\
\hline Type 2 diabetics & $59(\mathrm{M} / \mathrm{F})$ in three groups & $40-65($ mean 61$)$ & $\begin{array}{l}\text { 0, } 4 \mathrm{~g} / \mathrm{d} \text { EPA, } 4 \mathrm{~g} / \mathrm{d} \text { DHA } \\
\text { (EPA EE or DHA EE capsules; } 6 \text { weeks) }\end{array}$ & $\begin{array}{l}=\text { CRP, IL-6, TNF- } \alpha \text {, von Willibrand } \\
\text { factor, tissue plasminogen activator } \\
\text { antigen, PAl-1 antigen, sP-selectin }\end{array}$ & 636,644 \\
\hline Healthy & $60(\mathrm{M} / \mathrm{F})$ in three groups & Mean $\sim 38$ & $\begin{array}{l}0,1 \cdot 6,5 \cdot 8 \mathrm{~g} / \mathrm{d} \text { EPA + DHA } \\
\text { (concentrated FO capsules; } 3 \text { years) }\end{array}$ & $\begin{array}{l}\text { Low dose: } \\
=\text { sVCAM- } 1 \text {, sP-selectin } \\
\downarrow \text { sICAM-1 (especially in women) } \\
\text { High dose: } \\
=\text { sICAM-1, sVCAM-1 } \\
\downarrow \text { sP-selectin }\end{array}$ & 625 \\
\hline Obese & $11(\mathrm{M})$ & Not given & $1.1 \mathrm{~g} / \mathrm{d}$ EPA + DHA (FO capsules; 6 weeks) & $=$ CRP, IL-6, sTNFR1, sTNFR2, PAI-1 & 164 \\
\hline Hyperlipidaemia & $\begin{array}{l}563 \text { (M) in four groups } \\
\text { (two received } n-3 \text { PUFA) }\end{array}$ & 64-76 (mean 70) & $0,2.4 \mathrm{~g} / \mathrm{d}$ EPA + DHA (FO capsules; 3 years) & $\begin{array}{l}=\text { sVCAM-1, sE-selectin, } \\
\text { von Willibrand factor, tissue plasminogen } \\
\text { activator antigen } \\
\downarrow \text { sICAM-1, thrombomodulin }\end{array}$ & 628 \\
\hline Healthy & $\begin{array}{l}93 \text { young }(\mathrm{M}) \text { and } 62 \text { older } \\
(\mathrm{M}) \text { in four groups }\end{array}$ & $\begin{array}{l}\text { Mean 24(SD 10) } \\
\text { Mean 61(SD 8) }\end{array}$ & $0,1 \cdot 35,2 \cdot 7,4 \cdot 05 \mathrm{~g} / \mathrm{d}$ EPA + DHA (EPA-rich oil; 12 weeks) & $\begin{array}{l}=\text { sICAM-1, sVCAM-1 } \\
\uparrow \text { sE-selectin (young only) }\end{array}$ & 622 \\
\hline Healthy & $141(\mathrm{M} / \mathrm{F})$ in two groups & Mean $\sim 47$ & $0,0.96 \mathrm{~g} / \mathrm{d}$ EPA + DHA (FO in soya milk; 12 weeks) & $=$ CRP, sTNFR1, sTNFR2 & 626 \\
\hline Overweight and insulin resistant & $116(F)$ in three groups & $21-69$ (mean 45) & $\begin{array}{l}0,0+\text { weight-loss programme, } 4 \cdot 2 \mathrm{~g} / \mathrm{d} \text { EPA }+\mathrm{DHA}+ \\
\text { weight-loss programme (concentrated FO } \\
\text { capsules; } 24 \text { weeks) }\end{array}$ & $\begin{array}{l}\uparrow \text { Adiponectin } \\
=\text { CRP, TNF- } \alpha, \text { IL-6 }\end{array}$ & 632 \\
\hline Healthy & $80(\mathrm{M} / \mathrm{F})$ in two groups & Mean $\sim 30$ & $0,1.5 \mathrm{~g} / \mathrm{d}$ DHA (DHA-rich algal oil; 4 weeks) & $\begin{array}{l}=\text { CRP, fibrinogen, PAI-1 activity, } \\
\text { von Willibrand factor }\end{array}$ & 641 \\
\hline Overweight type 2 diabetics & $27(F)$ in two groups & Mean 55 & $0,1.8 \mathrm{~g} / \mathrm{d}$ EPA + DHA (FO capsules; 8 weeks) & $\begin{array}{l}=\text { IL- } 6, \text { TNF- } \alpha, \text { SAA, adiponectin } \\
\downarrow \text { PAl- } 1 \text { activity, inflammatory gene } \\
\text { expression in adipose tissue }\end{array}$ & 630 \\
\hline Overweight and obese & $30(F)$ in two groups & Not given & $\begin{array}{l}\text { 0, } 4.2 \mathrm{~g} / \mathrm{d} \text { EPA + DHA (DHA-rich TAG capsules; } \\
12 \text { weeks; cross-over) }\end{array}$ & $\begin{array}{l}=\text { Sialic acid, fibrinogen, PAl-1 activity } \\
\downarrow \text { CRP, IL-6 }\end{array}$ & 621 \\
\hline
\end{tabular}


Dietary fibre. In NHANES 1999-2000 in the USA, dietary fibre intake was inversely associated with serum CRP concentrations (OR 0.49 for $32 \mathrm{~g} / \mathrm{d} v .5 \cdot 1 \mathrm{~g} / \mathrm{d}$ ) in diabetic women $(n 3920)^{(677)}$, as well as in subjects with diabetes, hypertension and obesity $(n 7891)^{(678)}$. Similar findings were reported in a cross-sectional study with healthy subjects ( $n$ 205) and in subjects with metabolic disorders ( $n$ 1653) in Italy ${ }^{(679)}$. In the Seasonal Variation of Blood Cholesterol Levels Study involving 524 subjects, who had multiple measurements of CRP and dietary intake, dietary total fibre and soluble and insoluble fibre were all inversely related to CRP concentration in the regression models adjusted for several traditional cardiovascular risk factors, current infection status and season of year ${ }^{(680)}$. In contrast, the same authors observed no correlation between dietary fibre intake and blood CRP concentrations with a single measurement at baseline in the Women's Health Initiative Observational Study ${ }^{(681)}$. However, there was an inverse association between fibre intake and IL-6 and sTNFR2 concentrations in this female population. In a study with diabetic males ( $n$ 780, Health Professionals' Follow-Up Study), cereal fibre was positively associated with adiponectin levels after controlling for a number of confounding factors ${ }^{(315)}$. King et al. ${ }^{(682)}$ conducted an intervention study with thirty-five lean normotensive and seventeen obese hypertensive adults that involved a randomised cross-over design. The two intervention diets constituted either the Dietary Approaches to Stop Hypertension (DASH) diet (naturally high in fibre i.e. $30 \mathrm{~g}$ fibre/d) or a fibre-supplemented usual diet $(30 \mathrm{~g}$ psyllium fibre/d) each for a 3-week period. Both diets caused a reduction in CRP concentration (14 and 18\%, respectively), although this was significant only in lean normotensive subjects in either intervention arm. The data generally support that dietary fibre intake is associated with reduced low-grade inflammation.

\section{Milk peptides}

Increased consumption of dairy products has been shown to have beneficial effects on plasma CRP and adiponectin concentrations in obese subjects ${ }^{(683)}$. This effect has been suggested to be partly explained by the intake of dairy protein-derived peptides. Indeed, the casein-derived peptides Ile-Pro-Pro and Val-Pro-Pro slightly, though not significantly, lowered CRP concentration in hypertensive subjects after 10 weeks $^{(684)}$. Whey protein-derived peptides lowered CRP concentration (after 6 weeks) in one study in hypertensive subjects $^{(685)}$, but not in another ${ }^{(686)}$. Minor dairy proteins and peptides, especially lactoferrin, also show anti-inflammatory effects in different models ${ }^{(687-689)}$, and hence there is potential for a variety of milk peptides to have anti-inflammatory effects, but there are no or insufficient human studies to allow evaluation of their efficacy.

\section{Micronutrients and phytochemicals}

Iron

The topic of $\mathrm{Fe}$ in relation to low-grade chronic inflammation is complex. Part of the complexity is related to the fact 


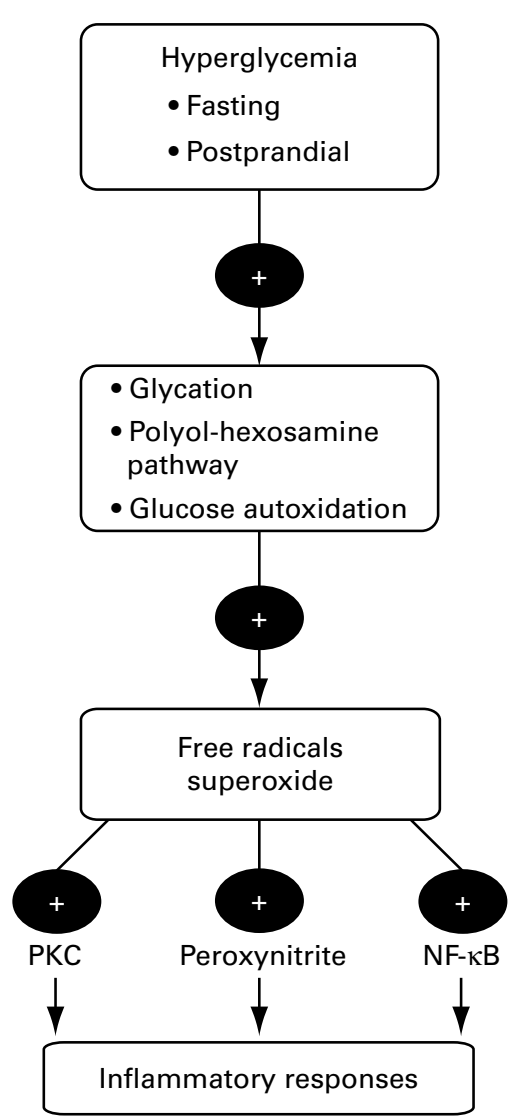

Fig. 8. Schematic representation of the general mechanisms by which hyperglycaemia can affect inflammation. PKC, protein kinase C. Reproduced with permission from Giugliano et al. ${ }^{(654)}$.

that Fe status assessment is confounded in the presence of inflammation ${ }^{(690-692)}$. Fe status assessment relies on a battery of laboratory tests spanning various stages of Fe deficiency. These tests include: serum ferritin, which generally indicates body Fe stores; soluble receptor of transferrin in serum (transferrin receptor) reflecting tissue Fe; serum transferrin, total Fe-binding capacity and transferrin saturation, which indicate Fe-deficient erythropoiesis; finally, red cell indices namely mean cell volume, $\mathrm{Hb}$ and haematocrit, which are considered functional $\mathrm{Fe}$ indices. Others have suggested the ratio of transferrin receptor:serum ferritin as an index of total body $\mathrm{Fe}$ stores ${ }^{(693)}$. Most tests of $\mathrm{Fe}$ status, however, are affected in response to subclinical inflammation and infections $^{(690-692)}$. Thus, it becomes difficult to study the relationship of Fe with any outcome of interest when inflammation is present. Recent studies have suggested that serum transferrin receptor remains unaffected in the presence of inflammation or infectious disease (reviewed in Ahluwalia ${ }^{(690)}$ and Northrop-Clewes ${ }^{(694)}$ ), thus allowing the examination of the role of Fe in conditions with an underlying inflammatory component.

Deficiency v. excess. Fe has been called a double-edged sword, as both Fe deficiency and excess can have deleterious effects ${ }^{(695)}$. Fe is important in immune/inflammatory responses including neutrophil activation, macrophage effector functions, and T-helper cell type-1 and -2 (Th1/Th2) patterns (reviewed in Weiss ${ }^{(695)}$ ). Fe deficiency is associated with alterations in the immune response and increased risk of infections ${ }^{(695-697)}$. On the other hand, because $\mathrm{Fe}$ is a redox-active transition metal, it may contribute to the production of ROS, oxidative stress and inflammation ${ }^{(698,699)}$, and thus Fe excess can augment the risk for diabetes and CVD. It is important to indicate that ROS are produced by free, and not bound, $\mathrm{Fe}^{(700)}$ and the body has evolved a metabolic system that minimises the availability of free $\mathrm{Fe}^{(698)}$. Most $\mathrm{Fe}$ in the body is not free, and is bound to proteins such as ferritin, transferrin and $\mathrm{Hb}$; thus, serum ferritin, transferrin, transferrin saturation and $\mathrm{Hb}$ measurements do not reflect the availability of 'free' or 'labile' Fe that is implicated in the production of ROS. In recent years, the measurement of non-transferring-bound labile $\mathrm{Fe}^{(701)}$ has been introduced; however, few studies have utilised this measurement to date due to technical, cost and standardisation issues.

Iron status and low-grade inflammation. There is some epidemiological evidence that higher Fe intake, particularly that of haem Fe, and higher Fe status are associated with increased risk of type 2 diabetes, atherosclerosis and $\mathrm{CHD}^{(702-714)}$, although not all of the literature is consistent. It is not clear whether the association of $\mathrm{Fe}$ intake and status with diabetes or CVD is mediated through the effects of $\mathrm{Fe}$ on inflammatory pathways. In a small study with thirty-one carbohydrate-intolerant patients, quantitative phlebotomy was used to induce Fe depletion to near-deficiency levels ${ }^{(715)}$. The induced Fe deficiency was associated with reductions in several CVD risk factors as well as in the inflammatory marker fibrinogen. While uncertainty exists as to whether Fe plays a causative role in the aetiology of low-grade inflammation and its associated pathologies, past and emerging evidence indicates that chronic low-grade inflammation is associated with poor Fe status in obese persons ${ }^{(716-721)}$. A concomitant improvement in $\mathrm{Fe}$ status (as measured by transferrin saturation) and decrease in inflammation (CRP and orosomucoid concentrations) has been observed after bariatric surgery intervention in morbidly obese women $^{(722)}$. In total, emerging evidence indicates that the low-grade inflammation of obesity may be associated with low Fe status; however, further investigation of this relationship is warranted.

A small number of studies have investigated the effects of increasing total Fe or haem Fe intake on markers of inflammation ${ }^{(723-725)}$. In a small study involving three healthy volunteers who received $120 \mathrm{mg} \mathrm{Fe} / \mathrm{d}$ for a week, there were no changes in circulating CRP concentration or leucocyte counts, or in urinary neopterin concentration ${ }^{(725)}$ Furthermore, postpartum Fe supplementation $(80 \mathrm{mg} / \mathrm{d})$ for 12 weeks in non-anaemic Fe-deficient women did not significantly alter CRP concentration or leucocyte counts ${ }^{(726)}$. In a small study in 8-11-year-old Guatemalan children who received twice the recommended daily amounts of Fe for 8 weeks ( $n$ 20) or placebo ( $n$ 20), no differences in CRP or orosomucoid concentrations were noted, although $\alpha-1$ antichymotrypsin levels were increased with the Fe supplement ${ }^{(724)}$. In another study examining the effect of increasing lean red meat intake, participants were either 
assigned to a control group (maintain their usual diet) or to partially replace energy from carbohydrate-rich foods with $200 \mathrm{~g} / \mathrm{d}$ of lean red meat for 8 weeks ${ }^{(723)}$. In this short intervention, increased red meat intake was not associated with increased oxidative stress or inflammation. Taken together, the evidence from these limited studies examining the effect of Fe supplementation or increased red meat intake on inflammatory markers suggests no major effect on inflammation; however, there is a need for further larger well-designed studies to clarify this effect.

\section{Vitamin D}

Various immune cells including monocytes/macrophages, dendritic cells, T-cells and B-cells can convert inactive vitamin $\mathrm{D}_{3}$ to its active form $\left(1,25(\mathrm{OH})_{2} \mathrm{D}_{3}\right)$, and these cells can also respond to the active vitamin $\mathrm{D}$ via its receptor which they express $^{(727)}$. It seems likely that vitamin D plays a paracrine modulatory role in the immune/inflammatory system ${ }^{(728)}$. Although a pro-inflammatory role of vitamin $\mathrm{D}$ has also been suggested ${ }^{(729)}$, epidemiological data show an association between vitamin $\mathrm{D}$ deficiency and increased risk of several inflammatory diseases including type 1 diabetes and atherosclerosis ${ }^{(730)}$. Vitamin D has several anti-inflammatory actions. It blunts the pro-inflammatory effects of AGE on endothelial cells, suggesting that it acts as an endogenous vascular protector counteracting the possible deleterious effects of $\mathrm{AGE}^{(731)}$. Vitamin D inhibits the proliferation of lymphocytes and induces their apoptosis ${ }^{(732)}$. In addition, vitamin $\mathrm{D}$ affects the expression of ICAM-1 on mononuclear cells and on endothelial cells, suggesting that it suppresses the recruitment of leucocytes to sites of inflammation ${ }^{(732)}$. In vitro, vitamin D modulates the pro-inflammatory profile of monocytes/macrophages from type 2 diabetic patients ${ }^{(733)}$. Vitamin D also suppresses TNF- $\alpha$ expression in monocytes/macrophages ${ }^{(734)}$ and down-regulates the expression of TLR2 and TLR4 in human monocytes ${ }^{(735-737)}$. Indeed, vitamin $\mathrm{D}$ primes monocytes to respond less effectively to bacterial cell wall components, most probably due to the aforementioned suppression of $\operatorname{TLR}^{(735)}$. Vitamin $\mathrm{D}$ analogues selectively inhibit the inducible cyclo-oxygenase- $2^{(738)}$, which could be viewed as an anti-inflammatory action. Exposure to vitamin $\mathrm{D}$ increases the rate of the de-phosphorylation of activated extracellular signal-regulated kinases ${ }^{(739)}$, a subset of the mammalian MAPK family involved in inflammatory processes. Serum vitamin D concentration was associated with leucocyte telomere length in 2160 women aged 18-79 years (mean age 49 years) and was inversely associated with CRP concentration $^{(740)}$. Within any tertile of vitamin D status, telomere length was longer in those with lower CRP concentrations, suggesting that inflammation plays a role in those processes associated with telomere shortening.

There are several studies looking at the association between vitamin D status (typically assessed as serum or plasma $\left.25(\mathrm{OH}) \mathrm{D}_{3}\right)$ and various markers of low-grade inflammation in different population subgroups. Shea et al. ${ }^{(741)}$ reported no significant association between plasma vitamin $\mathrm{D}$ concentration and concentrations of CRP, fibrinogen, TNF- $\alpha$, IL-6,
sTNFR2, sICAM-1, MCP-1, sP-selectin and sCD40 ligand in almost 1400 American adults with a mean age of 59 years. Likewise, there was no association of vitamin D status with serum CRP concentration in $650 \mathrm{Amish}^{(742)}$ or with CRP, sICAM-1, MCP-1, IFN- $\gamma$, IL-2, IL-4, IL-5, IL-10, IL-12, IL-13 or IL-17 concentrations in 437 overweight adults ${ }^{(743)}$. Using data from over 6500 British adults aged 45 years, Hypponen et $a l .{ }^{(744)}$ reported that the significant inverse associations between serum $25(\mathrm{OH}) \mathrm{D}_{3}$ concentration and serum CRP and fibrinogen were lost after adjustment for confounding factors. Among 261 healthy men and women, plasma $25(\mathrm{OH}) \mathrm{D}_{3}$ was not correlated with resistin, adiponectin or IL-18 concentrations but was inversely correlated with leptin concentration $^{(745)}$. Among forty-four morbidly obese subjects, $25(\mathrm{OH}) \mathrm{D}_{3}$ did not correlate with leptin, resistin, adiponectin or IL-18. Jablonski et al. ${ }^{(746)}$ examined the inflammatory phenotype of endothelial cells collected from the antecubital vein of middle-aged and older subjects: endothelial cell expression of NF- $\kappa \mathrm{B}$ and of IL- 6 were both higher in vitamin D-deficient subjects, and IL-6 expression was inversely related to $25(\mathrm{OH}) \mathrm{D}_{3}$ concentration. Using data pooled from thirty-six healthy subjects, twenty-four type 1 diabetics and twenty-six type 1 diabetics with microvascular complications, Devaraj et $a l .{ }^{(747)}$ found significant inverse relationships between serum $25(\mathrm{OH}) \mathrm{D}_{3}$ and $\mathrm{CRP}$ concentration, monocyte NF-кB activation and TLR4 expression. Most recently, NHANES data for 5867 adolescents aged 12-19 years showed no relationship between serum $25(\mathrm{OH}) \mathrm{D}_{3}$ and CRP concentrations ${ }^{(748)}$. Thus, association studies have consistently found little, if any, association between vitamin D status and circulating markers of inflammation. However, two studies that investigated cellular markers of inflammation both reported an anti-inflammatory effect of vitamin $\mathrm{D}^{(747,748)}$.

Most intervention studies with vitamin $\mathrm{D}$ have failed to identify a reduction in markers of low-grade inflammation ${ }^{(744,749,750)}$. These studies each had a different design. Pittas et al. ${ }^{(749)}$ provided $700 \mathrm{IU}(17.5 \mu \mathrm{g})$ vitamin $\mathrm{D}_{3}$ plus $500 \mathrm{mg}$ calcium citrate daily to adult non-diabetics for 3 years in a double-blind, randomised, controlled trial and found no effect on plasma CRP or IL-6. Witham et al. ${ }^{(751)}$ provided $100000 \mathrm{IU}(2500 \mu \mathrm{g})$ vitamin $\mathrm{D}_{2}$ or placebo to elderly patients with systolic heart failure at study entry (week 0) and after 10 weeks and found no effect on plasma TNF- $\alpha$ at 10 or 20 weeks. Jorde et al. ${ }^{(743)}$ provided 40000 or $20000 \mathrm{IU}$ (1000 or $500 \mu \mathrm{g}$ ) vitamin $\mathrm{D}_{3}$ per week or placebo to overweight subjects for 1 year; there was no difference between groups in the changes in concentrations of CRP, sICAM-1, MCP-1, IFN- $\gamma$, IL-2, IL-4, IL-5, IL-10, IL-12, IL-13 or IL-17 over the course of the study. Thus, these intervention studies suggest little anti-inflammatory action of vitamin D in the sorts of subjects studied. However, compared with placebo, vitamin $\mathrm{D}(3300 \mathrm{IU}(82.5 \mu \mathrm{g}) / \mathrm{d}$ for 12 months) resulted in a decrease (by $10 \%$ ) in TNF- $\alpha$ concentration in overweight subjects on a weight-reduction programme ${ }^{(752)}$. This finding suggests that vitamin $\mathrm{D}$ dose, the nature of the supplementation regimen and the health status of the individuals under study, as well as starting vitamin D status, may all be important factors in determining the effect of supplemental vitamin D. 


\section{Antioxidant vitamins (vitamin C, vitamin $E$ and} carotenoids)

Vitamin C is a potent water-soluble antioxidant. Ascorbate is the active form of vitamin $\mathrm{C}$ and exerts antioxidant function. Upon its action as an antioxidant, ascorbate is oxidised to dehydroascorbate which can be reduced back to ascorbate by the oxidation of reduced glutathione to glutathione disulfide. Ascorbate is present at high concentrations in leucocytes, suggesting a significant role in inflammation and in protection against oxidative damage. Patients with the metabolic syndrome or diabetes showed decreased vitamin $\mathrm{C}$ status and increased lipid peroxidation ${ }^{(753-756)}$. These findings could be explained by increased oxidative stress as a result of diabetes leading to consumption of ascorbate or a role of low vitamin $\mathrm{C}$ status as a risk factor for the development of diabetes. Obese subjects have lower plasma vitamin $\mathrm{C}$ concentrations than non-obese, and obesity was associated with moderately elevated CRP concentrations ${ }^{(757)}$. In another study, low plasma vitamin $\mathrm{C}$ concentrations were related to central fat distribution, independent of $\mathrm{BMI}^{(758)}$.

Vitamin $\mathrm{E}$ is an umbrella term for a number of tocopherols and tocotrienols, although dietary vitamin $\mathrm{E}$ mainly consists of $\alpha$ - and $\gamma$-tocopherols. Vitamin $\mathrm{E}$ is a potent chain-breaking antioxidant that acts mainly in the lipid phase and interrupts the chain reaction of lipid peroxidation and, consequently, prevents the propagation of free radical-initiated reactions. There are differences in the antioxidant activity between $\alpha$ - and $\gamma$-tocopherols. In vitro, vitamin $\mathrm{E}$ exerts a range of anti-inflammatory actions with regard to the production of pro-inflammatory cytokines and eicosanoids and adhesive interactions of monocytes with endothelial cells ${ }^{(750,759-761)}$. There is growing evidence that $\gamma$-tocopherol, in contrast to $\alpha$-tocopherol, exerts anti-inflammatory properties which can be explained by an unsubstituted position on the chromanol ring providing $\gamma$-tocopherol with the ability to trap reactive $\mathrm{N}$ species and subsequent formation of 5-nitro- $\gamma$-tocopherol $^{(762,763)}$. Supplementation with $\alpha$-tocopherol decreases $\gamma$-tocopherol concentrations ${ }^{(764,765)}$ due to a preference of the $\alpha$-tocopherol transfer protein in the liver for $\alpha$-tocopherol. This results in increased metabolism to carboxyethyl-hydroxychroman derivatives and excretion of the metabolites in the urine ${ }^{(766)} \cdot \gamma$-Tocopherol and $\gamma$-carboxyethyl-hydroxychroman exert actions that are not shared by $\alpha$-tocopherol and $\alpha$-carboxyethyl-hydroxychroman ${ }^{(767)}$

Carotenoids include, among others, $\alpha$-carotene, $\beta$-carotene, lycopene, $\beta$-cryptoxanthin, lutein and zeaxanthin. They are highly prevalent in red, yellow and green vegetables and fruits. Carotenoids exert antioxidant properties and some of them serve as provitamin A. Among 3258 healthy men (age 68 (SD 5) years; BMI $26.7(\mathrm{SD} 3.6) \mathrm{kg} / \mathrm{m}^{2}$ ), dietary vitamin $\mathrm{C}$ intake and plasma vitamin $\mathrm{C}$ concentrations were both inversely associated with CRP concentrations ${ }^{(329)}$. Among 5181 subjects from the MESA aged 45-84 years, intakes of vitamin $\mathrm{C}$, vitamin $\mathrm{E}$ or $\beta$-carotene were not associated with CRP, IL- 6 or fibrinogen concentrations after adjusting for a number of factors ${ }^{(768)}$. Recent data from the Womens' Health Study reported that higher plasma concentrations of $\alpha$ - and $\beta$-carotene were associated with lower plasma CRP concentrations $^{(769)}$. Similar results were reported from NHANES III for carotenoids and $\mathrm{CRP}^{(770,771)}$. However, the use of latex-enhanced nephelometry instead of a high-sensitivity ELISA, and the fact that $74 \%$ of the population had levels below the assay's detection limit, limits the interpretation of their results. In another study, dietary intakes of vitamin $C$, vitamin $E$ and $\beta$-carotene did not predict the concentrations of CRP, IL- 6 or TNF- $\alpha$ in normal-weight, overweight and obese Swiss children aged 6-14 years, but they were significant predictors of leptin concentrations ${ }^{(772)}$. Using data from a small number of middle-aged (mean age 44 years) vegetarians and omnivores (n 30 per group), plasma vitamin $\mathrm{C}$ concentrations were found to be inversely associated with CRP concentrations ${ }^{(295)}$. In 379 Dutch adults, serum lutein and lycopene concentrations were inversely associated with sICAM-1 concentrations, serum $\beta$-carotene with total blood leucocyte numbers and CRP concentrations, serum vitamin $\mathrm{C}$ with CRP concentrations, while plasma $\alpha$ tocopherol concentrations were positively associated with CRP concentrations ${ }^{(773)}$. Among 437 Japanese subjects, serum $\beta$-carotene concentrations were positively associated with adiponectin concentrations ${ }^{(774)}$. Other associations among $\alpha$ - and $\beta$-carotene and CRP and IL- 6 concentrations were lost after adjustment for confounding factors. A larger Japanese study ( $n 778$ men and 1404 women) reported an inverse association between serum vitamin $\mathrm{C}$ and CRP concentrations after adjustment for confounders ${ }^{(775)}$. The association was strongest in non-smokers, in non-overweight women and in postmenopausal women. Recently, plasma vitamin C concentrations have been reported to be inversely associated with plasma CRP concentrations in a small number of lean and obese men ( $n 8$ per group) with a mean age of 21 years $^{(776)}$. In patients with coronary artery disease, plasma concentrations of $\beta$-carotene, but not lycopene, were inversely correlated with plasma CRP concentrations ${ }^{(777)}$. In one prospective study with young normal-weight adults, a high intake of carotenoids ( $\alpha$-carotene, $\beta$-carotene, zeaxanthin/ lutein and $\beta$-cryptoxanthin) was inversely related to plasma CRP and ICAM concentrations after 7 and 15 years of follow-up ${ }^{(778)}$. In a second prospective study, a high intake of carotenoids in elderly subjects (BMI 28.8 (SD 6.8) $\mathrm{kg} / \mathrm{m}^{2}$ ) was associated with lower plasma IL-6 concentrations, while $\alpha$-tocopherol did not correlate significantly ${ }^{(779)}$. Among 704 70-year-old Swedish men, dietary intakes of vitamin $\mathrm{C}$ and $\alpha$-tocopherol, but not of $\beta$-carotene, were inversely associated with CRP and IL-6 concentrations measured 7 years after the dietary information was collected ${ }^{(780)}$. Thus, overall, crosssectional and prospective studies fairly consistently demonstrate that a higher intake and status of vitamin $\mathrm{C}$, vitamin $\mathrm{E}$ and carotenoids is associated with lower levels of low-grade inflammation

Taking $1 \mathrm{~g}$ of vitamin $\mathrm{C}$ or $533 \mathrm{mg}$ of $\alpha$-tocopherol before consuming a high-fat test meal blunted the acute CRP response to the meal ${ }^{(781)}$. Plasma 8 -iso PGF2 $\alpha$ and MCP-1 concentrations decreased after consumption of $72 \mathrm{mg} / \mathrm{d}$ of vitamin $\mathrm{C}$ from a vegetable soup ${ }^{(634)}$; concentrations of TNF- $\alpha$, IL- $1 \beta$ and IL- 6 did not change. Vitamin C $(1 \mathrm{~g} / \mathrm{d}$ for 
$14 \mathrm{~d}$ ) did not alter sICAM-1 concentrations or markers of monocyte and neutrophil activation (neopterin and elastase, respectively) in smokers or non-smokers ( $n 20$ of each) ${ }^{(782)}$. Likewise, vitamin C supplements $(250 \mathrm{mg}$ three times per week for 2 months) did not alter plasma CRP concentrations in thirty-three chronic haemodialysis patients ${ }^{(783)}$. Tomato juice (delivering $20.6 \mathrm{mg}$ lycopene/d) was compared with tomato juice fortified with vitamin C (delivering $435 \mathrm{mg}$ vitamin $\mathrm{C} / \mathrm{d}$ ) over a 2 -week intervention period: the vitamin C-enriched juice did not affect plasma CRP, TNF- $\alpha$ or IL-1 $\beta$ concentrations in healthy volunteers ${ }^{(784)}$. Vitamin $\mathrm{C}(1 \mathrm{~g} / \mathrm{d}$ for 6 months) lowered sP-selectin, but not sVCAM-1, sICAM-1 or sE-selectin concentrations in patients with chronic degenerative aortic stenosis ${ }^{(785)}$; combining vitamin $\mathrm{C}$ with $\alpha$-tocopherol $(267 \mathrm{mg} / \mathrm{d})$ lowered sICAM-1 concentrations. Smokers given $515 \mathrm{mg}$ vitamin C/d for 2 months had a $24 \%$ reduction in plasma CRP concentrations ${ }^{(786)}$. A mixture of vitamin $\quad C$ with $\alpha$-tocopherol $(371 \mathrm{mg} / \mathrm{d}), \quad \gamma$-tocopherol $(171 \mathrm{mg} / \mathrm{d})$, mixed tocotrienols $(252 \mathrm{mg} / \mathrm{d})$ and $\alpha$-lipoic acid $(95 \mathrm{mg} / \mathrm{d})$ resulted in a smaller effect $(4.7 \%$ reduction that was not significant), suggesting that tocopherols, tocotrienols or $\alpha$-lipoic acid, or the combination, prevents the anti-inflammatory effect of vitamin C. Vitamin C ( $2 \mathrm{~g} / \mathrm{d}$ for 4 weeks $)$ did not affect serum IL-6, IL-1b, sVCAM-1 or sICAM-1 concentrations in smokers, but combining $533 \mathrm{mg} / \mathrm{d} \alpha$-tocopherol with the vitamin $C$ decreased the concentrations of all four inflammatory mediators; the combination of vitamin $\mathrm{C}$ and $267 \mathrm{mg} / \mathrm{d} \alpha$-tocopherol was without effect ${ }^{(787)}$.

$\alpha$-Tocopherol $(800 \mathrm{mg} / \mathrm{d}$ for 3 months) decreased plasma CRP concentrations in type 2 diabetics $^{(788)}$. Dalgard et al. ${ }^{(789)}$ examined the effect of a $28 \mathrm{~d}$ intervention with orange and blackcurrant juice $(500 \mathrm{ml} / \mathrm{d})$, vitamin E (15 mg RRR- $\alpha$ tocopherol/d) or the combination of the two in patients with peripheral vascular disease. The juice but not vitamin $\mathrm{E}$ decreased CRP (by 11\%) and fibrinogen, but there was no effect on IL-6 or PAI-1. In a randomised, placebo-controlled, double-blind trial, subjects with the metabolic syndrome received $800 \mathrm{mg} / \mathrm{d} \quad \alpha$-tocopherol, $800 \mathrm{mg} / \mathrm{d} \quad \gamma$-tocopherol, a combination of both $(800 \mathrm{mg} / \mathrm{d}$ each) or placebo; there was a decrease in CRP concentrations only in the combined $\alpha$ - and $\gamma$-tocopherol supplementation group, while TNF- $\alpha$ decreased with $\alpha$-tocopherol alone or in combination with $\gamma$-tocopherol ${ }^{(790)}$. Biomarkers of oxidative stress decreased with $\alpha$-tocopherol, $\gamma$-tocopherol or the combination, while nitrotyrosine, a biomarker of nitrative stress, decreased with $\gamma$-tocopherol alone or in combination with $\alpha$-tocopherol $^{(646-648)}$. In another randomised, placebo-controlled, double-blind trial, overweight and obese and normal-weight young adults completed a standardised $30 \mathrm{~min}$ cycle exercise bout before and after 8 weeks of supplementation with 800 $\mathrm{IU} / \mathrm{d}$ of vitamin $\mathrm{E}$ (form not stated), $500 \mathrm{mg} / \mathrm{d}$ of vitamin $\mathrm{C}$ and $10 \mathrm{mg} / \mathrm{d}$ of $\beta$-carotene ${ }^{(791)}$. Adiponectin concentrations were increased by $22 \%$ in the overweight and by $3 \%$ in the normal-weight group receiving the supplement, but was reduced in the placebo group. Changes in circulating IL-6 concentrations during exercise were lower in the supplemented groups, as were the changes in lipid hydroperoxides $^{(791)}$. In contrast, a double-blind, placebo-controlled trial, in which type 2 diabetics received $500 \mathrm{mg} / \mathrm{d} \alpha$-tocopherol or mixed tocopherols containing $315 \mathrm{mg} / \mathrm{d} \quad \boldsymbol{\gamma}$-tocopherol, reported no effect on CRP, IL-6, TNF- $\alpha$ or MCP-1 concentrations, although there was a decrease in plasma $\mathrm{F}_{2}$-isoprostane concentrations, a biomarker of lipid peroxidation, in the mixed tocopherol group ${ }^{(792)}$. A small study conducted in healthy subjects ( $n$ 12) and patients with CHD ( $n$ 12) investigated the effect of increasing intake of $\alpha$-tocopherol (100, 200 and $400 \mathrm{mg} / \mathrm{d}$ each for 3 weeks sequentially) ${ }^{(793)}$. At $200 \mathrm{mg} / \mathrm{d}$, plasma CRP, IL-6 and fibrinogen concentrations were decreased in the CHD patients. A long-term (3 years) intervention with the combination of vitamin C $(500 \mathrm{mg} / \mathrm{d})$ and $\alpha$ tocopherol $(182 \mathrm{mg} / \mathrm{d})$ in 45-69-year-old men did not alter CRP, TNF- $\alpha$ or IL- 6 concentrations ${ }^{(794)}$. In one study, fifteen children with familial hyperlipidaemia given vitamins $\mathrm{C}$ plus E $(500 \mathrm{mg} / \mathrm{d}$ and $400 \mathrm{IU} / \mathrm{d}$, respectively) for 6 weeks showed no changes in CRP concentration ${ }^{(795)}$. In another study, six weeks' supplementation with vitamin $\mathrm{C}(1 \mathrm{~g} / \mathrm{d})$ plus vitamin E $(300 \mathrm{mg} / \mathrm{d}$ RRR- $\alpha$-tocopherol) did not alter the magnitude of the increase in circulating CRP, TNF- $\alpha$ and IL-6 concentrations seen after running a marathon ${ }^{(796)}$. Older men given $1 \mathrm{~g} / \mathrm{d}$ of vitamin C plus $1000 \mathrm{mg} / \mathrm{d}$ vitamin $\mathrm{E}$ for 4 weeks showed a decrease in plasma TNF- $\alpha$ concentrations ${ }^{(797)}$. Recently, a mixture of fruit-derived antioxidants has been found not to alter CRP or IL-6 concentrations over 12 weeks in type 2 diabetics ${ }^{(798)}$. In an intervention study with carotenoid-rich vegetables and fruits, plasma concentrations of $\alpha$ - and $\beta$-carotene, but not other carotenoids, were inversely associated with plasma CRP concentrations in healthy normalweight men ${ }^{(345)}$. Lycopene $(80 \mathrm{mg} / \mathrm{d}$ for 1 week) did not affect plasma CRP, sVCAM-1 or sICAM-1 concentrations in men and women with a mean age of 23 years ${ }^{(799)}$.

Thus, in contrast to observational studies that provide a fairly consistent picture of an anti-inflammatory effect of vitamin $\mathrm{C}$, vitamin $\mathrm{E}$ and carotenoids, intervention studies using supplements of these antioxidant vitamins either alone or in various combinations provide a less consistent set of observations. A number of studies do demonstrate a reduction in the concentrations of circulating inflammatory markers in a variety of subgroups of individuals, including the overweight persons and diabetics, but quite a number of studies did not find an effect. The lack of consistency may be related to differences in: dose of antioxidant used (however, typically the doses used are much greater than those that can be readily achieved in the diet and are therefore much greater than would have been present in the diets of subjects investigated in the observational studies); duration of treatment (typically a few weeks to a few months); sample size which has often been small; characteristics of the populations studied (e.g. age, healthy $v$. diseased, type of disease, smokers $v$. non-smokers); background diet; interactions among the different antioxidant vitamins used, since they may have different anti-inflammatory potencies and some may even act in a pro-inflammatory way under certain conditions; degree of oxidative stress present. One other factor that has recently been identified is genetic differences among individuals, which may have an impact on the ability of antioxidants to exert an anti-inflammatory effect. Such an effect has been 
identified by Belisle $e t a l{ }^{(800)}$ who showed that the ability of $\alpha$-tocopherol to lower the LPS-stimulated production of TNF- $\alpha$ by whole blood was determined in part by polymorphisms within the TNF- $\alpha$ gene. Thus, it is possible that a greater or lesser anti-inflammatory effect of antioxidant vitamins will be observed in people with different genotypes related to inflammatory processes. Clearly, this needs greater exploration in properly designed studies.

\section{Flavonoids}

Polyphenols are secondary metabolites of plants involved in pigmentation, reproduction and protection against pathogens. There are more than 8000 known polyphenolic substances sharing a common chemical structure (hydroxyl group on an aromatic ring) with different constituents. Flavonoids are the most abundant polyphenols present in the human diet, and they can be divided into several classes according to different constituents such as flavanones, flavones, flavanols and flavonols. They can be found in almost all plant foods and, among the flavonols, myricetin, kaempferol and quercetin are the most representative, while catechins are the most abundant flavanols contained in tea leaves. Flavanones are mainly represented in the diet by taxifolin, naringinin and hesperitin. The main sources of flavanones are citrus fruits. Flavones are less common. In addition to these, other classes of flavonoids are present in the diet such as proanthocyanidins and their oligomers.

The intake of flavonols and flavones was not correlated with plasma concentrations of CRP or IL- 6 in healthy women (BMI $\left.25 \cdot 8-26 \cdot 2 \mathrm{~kg} / \mathrm{m}^{2}\right)^{(801)}$. While this study used a rather limited flavonoid database, a more recent crosssectional study applying a comprehensive flavonoid database reported anti-inflammatory effects associated with a high flavonoid intake: total flavonoid, flavonol and anthocyanidin intakes were inversely associated with plasma CRP concentrations $^{(323)}$. Data from the Nurses' Health study were used to assess the relationship between flavonoid intake and biomarkers of inflammation ${ }^{(802)}$ : intake of six flavonoid subclasses (flavonols, flavones, flavanones, flavan-3-ols, anthocyanidins and polymeric flavonoids) was assessed using a FFQ administered in 1990 and blood samples collected in 1989-90 were used to measure concentrations of CRP, IL-6, IL-18, sTNFR2, sVCAM-1 and sE-selectin. Multivariate-adjusted mean plasma IL-18 concentrations were lower (by 9, 11 and $8 \%$, respectively) for women in the highest intake quintile of flavones, flavanones and total flavonoids compared with those in the lowest quintiles. Multivariate-adjusted geometric plasma sVCAM-1 concentrations were lower by $4 \%$ in women in the highest intake quintile of flavonol compared with those in the lowest quintile. Thus, the study suggests that higher intakes of selected flavonoid subclasses are associated with modestly lower concentrations of some inflammatory biomarkers.

In a randomised human intervention trial with healthy normal-weight adults, supplementation with a bilberry extract providing $300 \mathrm{mg}$ anthocyanins/d (equal to $100 \mathrm{~g}$ of fresh bilberries) reduced plasma concentrations of several
NF-кB-induced pro-inflammatory cytokines (IL-8, RANTES and IFN- $\alpha)^{(337)}$. In addition, NF-кB-inducing cytokines (IL-4 and IL-13) tended to differ from controls, while plasma CRP concentrations did not change ${ }^{(337)}$. In subjects who had survived myocardial infarction and had received statin therapy for at least 6 months, supplementation with a chokeberry flavonoid extract for 6 weeks significantly decreased CRP and MCP-1 concentrations, while adiponectin was significantly increased ${ }^{(803)}$. In contrast, although the consumption of black tea resulted in increased plasma catechin concentrations, these were not associated with changes in plasma CRP concentrations in healthy overweight and obese adults $^{(389)}$. A similar negative effect on CRP was observed in a double-blind, placebo-controlled, cross-over study with healthy adults (mean BMI $25.8 \mathrm{~kg} / \mathrm{m}^{2}$ ) who were supplemented with a sea buckthorn flavonol extract during 4 weeks $^{(804)}$. Quercetin (50, 100 or $150 \mathrm{mg} / \mathrm{d}$ for 2 weeks) did not affect serum concentrations of TNF- $\alpha$ in healthy adults $^{(805)}$. Overweight or obese subjects aged 25-65 years with metabolic syndrome traits received $150 \mathrm{mg}$ quercetin/d in a double-blind, placebo-controlled, cross-over trial with 6-week treatment periods separated by a 5-week washout period: quercetin did not affect CRP or TNF- $\alpha$ concentrations compared with placebo ${ }^{(806)}$. Quercetin $(1 \mathrm{~g} / \mathrm{d}$ for $21 \mathrm{~d})$ failed to attenuate muscle inflammation in ultramarathon runners ${ }^{(807)}$ and in trained cyclists ${ }^{(808,809)}$. However, leucocyte IL-8 and IL-10 mRNA were significantly reduced, indicating that a high dose of quercetin may target blood cells but not the muscle tissue.

\section{Phyto-oestrogens}

Genistein is an isoflavone and a phyto-oestrogen which primarily occurs in soyabeans. Native phyto-oestrogens exist as glycosides, while in experimental studies, mostly the aglycones have been used. In two intervention studies with healthy postmenopausal women, the intake of genistein (54 or $40 \mathrm{mg} / \mathrm{d}$ ) for 6 months did not significantly affect plasma CRP concentrations ${ }^{(810,811)}$. The intake of soya either high or low in isoflavones for 1,2 or 4 months had also no effect on CRP, SAA or TNF- $\alpha$ concentrations in hypercholesterolaemic men or in postmenopausal women ${ }^{(356,359,362)}$. In obese postmenopausal women, the combination of exercise with a soya isoflavone supplement (duration 6 months) did not decrease plasma CRP concentration compared with exercise + placebo ${ }^{(812)}$. Consumption of a soya isoflavone-enriched cereal bar $(50 \mathrm{mg} / \mathrm{d})$ for 8 weeks by postmenopausal women had no effect on CRP or other plasma markers of inflammation $^{(813)}$. A higher intake of soya isoflavones $(114 \mathrm{mg} / \mathrm{d})$ for 3 months also did not reduce serum CRP or sE-selectin concentrations in postmenopausal women ${ }^{(814)}$. In another study, isoflavone-rich soya $(107 \mathrm{mg} / \mathrm{d}$ as aglycone; $50 \%$ as genestein) for 6 weeks did not affect sVCAM-1, sICAM-1 or sE-selectin concentrations in healthy postmenopausal women compared with isoflavone-poor soya ${ }^{(815)}$. In contrast, a randomised, controlled study providing pasta naturally enriched with isoflavone aglycones $(33 \mathrm{mg} / \mathrm{d})$ to overweight hypercholesterolaemic subjects reported significantly 
reduced plasma CRP concentrations, which returned to baseline when subjects were switched to conventional pasta $^{(816)}$. Overall, the majority of the studies with soyaderived isoflavones did not observe a significant effect on inflammatory processes in human subjects. In contrast, a lignan complex $(500 \mathrm{mg} / \mathrm{d})$ isolated from flax given to healthy postmenopausal women significantly reduced plasma CRP concentration during the 6-week placebo-controlled intervention. No significant differences were found for IL-6, TNF- $\alpha$, sICAM-1, sVCAM-1 or MCP-1 concentrations ${ }^{(817)}$. In a recent study, flaxseed-derived lignans $(360 \mathrm{mg} / \mathrm{d})$ lowered CRP concentrations in type 2 diabetic women but not in men over 12 weeks compared with placebo, with no effect on IL-6 concentrations ${ }^{(818)}$. A cross-sectional study in 242 men and postmenopausal women in Northern Italy revealed inverse associations between dietary intake of the lignans matairesinol and secoisolariciresinol and plasma concentrations of $\operatorname{sICAM}-1^{(819)}$.

\section{Other factors}

\section{Gut microbiota and probiotics}

Probiotics are live micro-organisms which, when consumed in adequate quantities, confer a health benefit on the host $^{(820,821)}$. One differential characteristic of probiotics compared with other micro-organisms is their ability to survive during gastrointestinal transit ${ }^{(822)}$. This allows them to interact with commensal microbiota and/or intestinal epithelial cells and also with gut-associated lymphoid cells, which results in the induction or modulation of a number of biological activities that can provide beneficial effects for health. The capacity of probiotics to modulate the mucosal immune system is regarded as one of the most obvious beneficial properties. The microbiota composition and the related local intestinal metabolism undergoes significant changes in various disease states that are characterised by chronic inflammation, such as inflammatory bowel disease ${ }^{(823)}$, colorectal cancer and obesity $^{(824,825)}$. Mice raised under germ-free conditions fail to develop experimental colitis, suggesting an important modulating role of intestinal microbiota on local inflammatory processes $^{(826)}$. Thus, there seems to be a strong association between the nature of the gut microbiota and inflammation.

A number of studies have investigated the effects of various probiotics on aspects of inflammation in a variety of subject and patient groups, as reviewed elsewhere ${ }^{(827)}$. However, relatively few studies have focused on circulating markers of inflammation in persons without an established inflammatory disease. In a small study in institutionalised elderly subjects (mean age 76 years), there was no effect of the combination of Bifidobacterium longum and Lactobacillus acidophilus $\left(8 \times 10^{9}\right.$ colony forming units (CFU) of each/d for $\left.28 \mathrm{~d}\right)$ on serum TNF- $\alpha$ concentration ${ }^{(828)}$. L. salivarius UCC118 $\left(10^{10}\right.$ CFU/d for 21 d) did not affect serum IL- $1 \alpha$, IL-1 $\beta$, IL-4, sIL-2R, sIL-6R, TNF- $\alpha$ or IFN- $\gamma$ concentrations in healthy adults aged $20-65$ years $^{(829)}$. Likewise, the combination of $L$. gasseri CECT5714 and L. coryniformis CECT5711 $\left(2 \times 10^{9}\right.$ CFU of each/d) with Staphylococcus thermophilus $\left(10^{8} \mathrm{CFU} / \mathrm{d}\right)$ for 2 or 4 weeks had no effect on serum TNF- $\alpha$ or IL-12 concentrations in healthy adults aged $23-43$ years ${ }^{(830,831)}$. Kekkonen et al. ${ }^{(832)}$ compared L. rhamnosus GG ATCC53103 $\left(1.6 \times 10^{10}\right.$ $\mathrm{CFU} / \mathrm{d})$ with Bifidobacterium animalis ssp. lactis Bb12 $\left(3.5 \times 10^{10} \mathrm{CFU} / \mathrm{d}\right)$ and Propionibacterium freudenreichii ssp. Shermani JS $\left(3.3 \times 10^{10} \mathrm{CFU} / \mathrm{d}\right)$ for 3 weeks in healthy subjects with a mean age of 44 years (range 23-58 years). There was no effect of serum TNF- $\alpha$, IL-6, IL-10 or IFN- $\gamma$ concentrations, but serum CRP concentration decreased in the L. rhamnosus group. Ouwehand et al. ${ }^{(833)}$ conducted a 6-month placebo-controlled trial with $B$. animalis ssp. lactis Bb12 $\left(10^{9} \mathrm{CFU} / \mathrm{d}\right)$ or the combination of B. longum $2 \mathrm{C}$ and B. longum $46\left(10^{9} \mathrm{CFU}\right.$ of each/d) in institutionalised elderly subjects (mean age 84 years). Although there were some changes over time, the groups did not differ in serum TNF- $\alpha$, IL-10 or TGF- $\beta$ concentrations. Thus, although there is the potential for probiotics to lower markers of chronic low-grade inflammation, intervention studies performed to date in human volunteers rarely demonstrate this effect.

\section{Prebiotics}

There are two recent definitions of prebiotics: 'a selectively fermented ingredient that allows specific changes, both in the composition and/or activity in the gastrointestinal microflora that confers benefits upon host well-being and health'(834), and 'a non-viable food component that confers a health benefit on the host associated with modulation of the microbiota, ${ }^{(835)}$. Typically, though not exclusively, prebiotics are carbohydrates including inulin-type fructans (including oligofructose, fructo-oligosaccharides), lactulose, galacto-oligosaccharides, xylo-oligosaccharides, D-tagatose, resistant starch, soyabean oligosaccharides, pectin, guar, carrageenan, konjac glucomannans, alginates and $\beta$-glucans from oat, barley and mushroom. Prebiotics escape digestion in the upper gastrointestinal tract and reach the large intestine virtually intact, where they are fermented by the microbiota and express their prebiotic activity ${ }^{(834)}$. The latter is most probably mediated through a quantitative increase in commensal bacteria (e.g. bifidobacteria and lactobacilli), which interact with other members of the gut microbiota. Further, changes in the microbiota enzyme activities, leading to a reduction of unfavourable substances that have an impact on disease risk, such as secondary bile acids, and the production of metabolites such as SCFA and vitamins also contribute to their impact on health and disease ${ }^{(834)}$. In high-fat-fed mice, oligofructose restored gut bifidobacteria, and normalised systemic endotoxin levels and the inflammatory state $^{(836)}$. Institutionalised elderly individuals (mean age 85 years) supplemented with oligofructose ( $8 \mathrm{~g} / \mathrm{d}$ for 3 weeks) showed increased faecal bifidobacteria counts and decreased expression of IL- 6 mRNA in blood monocytes ${ }^{(837)}$. In another study, in poorly nourished elderly subjects (age $>70$ years), oligofructose $(1.95-3.9 \mathrm{~g} / \mathrm{d}$ for 12 weeks as part of a nutritional supplement) had no effect on plasma TNF- $\alpha$ or sIL-6R concentrations ${ }^{(838)}$. Prebiotics have been studied in the context of inflammatory conditions, especially those involving the gastrointestinal tract, as reviewed elsewhere ${ }^{(839)}$. There is 
some evidence of beneficial effects but results are inconsistent. There are few studies of prebiotics and circulating inflammatory markers in the context of chronic low-grade inflammation. Thus, it is premature to draw conclusions about this relationship.

\section{Hydration}

When fluids are consumed, water distributes between intraand extracellular compartments according to osmotic load. When water enters the cells, they swell and when water is lost from the cells, for example, during dehydration, the cells will shrink. The maintenance of adequate cell volume can have a profound effect on protein function and cellular performance. Cells employ an array of mechanisms to maintain cell volume constancy, including altered transport across the cell membrane and metabolism. Hormones and mediators may modify the activity of these cell volume regulatory mechanisms and thus influence cell volume-sensitive functions. Cell volume regulatory mechanisms, therefore, participate in the signalling of those hormones and mediators ${ }^{(840)}$. Many metabolic pathways are sensitive to cell volume ${ }^{(841)}$, as a result of activation, inhibition or altered expression of enzymes. Cellular volume has the potential to have an impact directly or indirectly on inflammation, but the effect of hydration status on low-grade inflammation is not well documented.

\section{Summary, conclusions and research gaps}

Inflammation is part of the normal host defence mechanism against infections. However, inflammatory mediators and the inflammatory response can be damaging to the host if not regulated appropriately and numerous diseases and conditions have an overt chronic inflammatory basis. It is now recognised that a lower level of inflammation, here termed chronic low-grade inflammation, can also persist and may be a cause of, or result from, the obese state. Adipose tissue releases many of the characteristic mediators of inflammation including some of the classic pro-inflammatory cytokines and chemokines, as well as adiponectin which is considered to be anti-inflammatory. The source of these mediators within adipose tissue is not clear, but infiltrating macrophages seem to be especially important in this regard, although adipocytes themselves express some of the inflammatory mediators. Obese people have higher circulating concentrations of many inflammatory markers (measured in the 'resting' state, i.e. in fasted blood), and these are believed to play a role in causing insulin resistance and in other metabolic disturbances of the metabolic syndrome and type 2 diabetes. Blood concentrations of inflammatory markers are lowered following weight loss, whether this is induced by diet or surgery, which most probably reflects the decrease in adipose tissue mass. In the hours following the consumption of a meal, there is an elevation in the concentrations of several inflammatory mediators in the bloodstream. This postprandial inflammatory response is greater in obese subjects and type 2 diabetics. Both high-glucose and high-fat meals induce postprandial inflammation, and it is exaggerated by a high meal content of AGE and partly ablated by the inclusion of certain antioxidants or antioxidant-containing foods within the meal. These latter observations link postprandial inflammation to oxidative stress. Physical activity decreases low-grade inflammation. Exercise itself is associated with transient and local inflammation (e.g. in muscle) that may, in fact, be important in inducing a protective and ultimately healthy anti-inflammatory response. In addition to the direct effect of foods and their constituents on postprandial inflammation, diet has an impact on chronic low-grade inflammation, manifested as the basal (i.e. fasting state) concentrations of inflammatory markers in the bloodstream, including cytokines, chemokines, acute-phase proteins, soluble adhesion molecules and cytokine receptors, etc. Effects of diet and dietary components on low-grade inflammation have been identified through cross-sectional, prospective and intervention studies. The former two study designs frequently involve large numbers of subjects, while the intervention studies often have a small sample size and a limited duration which might limit their ability to identify effects; compliance may also be a limitation of intervention studies. Healthy eating patterns such as the Mediterranean diet, vegetarian diets and adherence to the Food Guide Pyramid are associated with lower concentrations of inflammatory markers; this is observed mainly from crosssectional and prospective studies, although intervention studies with the Mediterranean diet have been positive. Among the components of healthy diets, whole grains, vegetables and fruits, and fish are all seen to be associated with lower inflammation. Strong evidence in favour of an antiinflammatory effect of tea (black or green), coffee (caffeinated or decaffeinated) and cocoa is lacking, despite positive effects on oxidative stress and the anti-inflammatory effects, mainly demonstrated in model systems (e.g. cell cultures), of components of these foods. Alcohol appears to have a 'U-shaped' effect on low-grade inflammation, with the most protective action (i.e. the lowest inflammatory marker concentrations) corresponding to one or two alcoholic drinks per $\mathrm{d}$. Heated meals high in AGE and ALE enhance oxidative stress and inflammation; intervention studies with low- and highAGE meals either acutely or chronically have associated AGE with increased inflammatory marker concentrations and show that these are decreased by meals low in AGE. However, AGE and ALE are also generated in vivo and also as a result of other, non-food-related, environmental exposures (e.g. smoking), and so the overall impact of foods on the burden of AGE and ALE is not currently clear; this is compounded by technical difficulties in measuring these complex chemical entities in foods and in body fluids. Dietary fatty acids also influence low-grade inflammation; best studied are PUFA. Available data indicate that SFA and trans-MUFA are pro-inflammatory, and that one isomer of conjugated linoleic acid may also be. Relative to SFA, PUFA are anti-inflammatory. Marine $n-3$ PUFA have the greatest anti-inflammatory potential. Hyperglycaemia induces both postprandial and chronic low-grade inflammation, acting in part through oxidative stress. Dietary fibre decreases low-grade inflammation. There may also be a role for milk peptides, but these have not been sufficiently evaluated. Vitamin D has the potential to reduce low-grade 
inflammation, with plausible mechanistic actions demonstrated in model systems. There is good evidence from both model systems and from human observational and intervention studies that vitamin $\mathrm{C}$, vitamin $\mathrm{E}$ and carotenoids decrease the concentrations of inflammatory markers. $\alpha$ - and $\gamma$-Tocopherols and the different carotenoids may have different anti-inflammatory properties and potencies. The majority of available evidence indicates that soya phyto-oestrogens and soya protein do not affect low-grade inflammation. There are likely to be many plant-derived substances that influence low-grade inflammation and which may have a role as part of a healthy 'anti-inflammatory diet'. Several studies of various probiotic bacteria have failed to demonstrate any consistent effects on markers of chronic low-grade inflammation, despite their apparent effectiveness in higher-grade inflammatory states. The effect of prebiotics on chronic low-grade inflammation is not clear and is underexplored.

The key conclusions are as follows:

(1) A state of chronic low-grade inflammation exists and this is exaggerated in obese and type 2 diabetic individuals.

(2) Adipose tissue plays a role in establishing the chronic low-grade inflammatory state because cells within that tissue can produce and release the mediators involved.

(3) Chronic low-grade inflammation is believed to increase the risk of insulin resistance, type 2 diabetes and CVD.

(4) Following consumption of a meal, there is a transient state of inflammation that is linked with oxidative stress.

(5) Hyperglycaemia or a high-fat meal promote postprandial inflammation.

(6) A healthy diet is associated with decreased low-grade inflammation.

(7) Important protective factors in the diet are whole grains, fibre, vegetables, fruits, fish, PUFA, especially marine $n$ - 3 PUFA, vitamin C, vitamin E and carotenoids.

(8) Plant-derived flavonoids are likely to be protective.

(9) Moderate alcohol consumption decreases low-grade inflammation.

(10) Dietary factors that promote inflammation are oxidised lipids, SFA and trans-fatty acids.

(11) Underexplored dietary factors include milk peptides, vitamin $\mathrm{D}$, probiotics and prebiotics.

A number of important research gaps were identified. Perhaps the most important is that there is no consensus regarding the inflammatory mediators which best represent chronic low-grade inflammation. Most studies have measured CRP, perhaps because it is long established, is linked to the risk of CVD and is routinely measured in clinical laboratories. However, it is not clear whether CRP is a 'better' marker of low-grade inflammation than any of the other mediators measured, which include a variety of cytokines, soluble cytokine receptors, chemokines, soluble adhesion molecules and so on. Furthermore, there has been little emphasis on anti-inflammatory mediators. An expert review of the area of inflammatory markers addressing the most valid and robust markers and including a consideration of anti-inflammatory factors is warranted. Identification of dietary components that promote or prevent postprandial inflammation and the underlying mechanisms involved is a further gap. The impact of many dietary components on chronic low-grade inflammation is underexplored in human intervention studies; such components include flavonoids and other phytochemicals, milk peptides, vitamin $\mathrm{D}$, probiotics and prebiotics. Further, even where dietary components have been fairly well explored through intervention studies, many of the studies have been small in size and they have reported on different inflammatory outcomes at different time points. For these components (whole grains, fibre, vegetables, fruits, fish, PUFA, especially marine $n$-3 PUFA, vitamin C, vitamin E and carotenoids), a better knowledge of the dose-response effect, the threshold dose (if any) and the time required for an effect to occur would all be valuable information. Such variations in study design may also explain the discordant findings of studies with tea, coffee and cocoa, and these dietary components require further investigation in the context of robust markers of inflammation, appropriate sample size and duration of exposure, and exploration of dose-response relationships. Oxidative stress and inflammation are strongly interlinked and effects of dietary components appear to frequently involve increased or decreased oxidative stress. In this regard, modified food components such as AGE and ALE may be very potent markers of oxidative and inflammatory stress, although their causal impact remains elusive. However, further exploration and greater understanding of this area is impeded by lack of agreed analytical methods for quantifying AGE in foods and in biological fluids, with inter-laboratory cross-validation, and by lack of discrimination between protein-bound and free AGE. Finally, the emerging area of the role of gene polymorphisms in influencing or even determining the effect of nutrients on markers of low-grade inflammation requires much greater exploration. Until these gaps are filled, our understanding of the interaction between diet and postprandial and chronic low-grade inflammation will remain incomplete.

\section{Acknowledgements}

This work was commissioned by the Metabolic Syndrome and Diabetes Task Force of the European Branch of the International Life Sciences Institute (ILSI Europe). Industry members of this task force are Ajinomoto Europe, Coca-Cola Europe, Danisco, Danone, Kellog Europe, Kraft Foods Europe and Schwabegroup. For further information about ILSI Europe, please email info@ilsieurope.be or call +32 2 7710014 . The opinions expressed herein and the conclusions of this publication are those of the authors and do not necessarily represent the views of ILSI Europe nor those of its member companies. Conflict of interest statement: P. C. C. has research funding from Beneo-Orafti, Beghin-Meiji, Abbott Nutrition and Vifor Pharma and is a consultant to Danone. F. B. was an employee of Cargill. T. B. was and J. O. is an employee of Nestlé. K. Cu. is an employee of Coca-Cola Europe. L. S. J. is an employee of ILSI Europe. M. L. is an employee of Danone. N. M. is an employee of Kraft Foods Europe. H. N. is an employee of Ajinomoto Europe. P. C. C., N. A., F. B., K. Cl., K. E., H. K., As. M., 
An. M., G. P., S. R., C. S., J. T., J. W. and B. M. W-R received an honorarium from ILSI Europe for their participation in this publication and/or reimbursement of their travel and accommodation costs for attending the related meetings. The remaining authors have no conflicts of interest. All authors contributed to discussions and had input into writing the article. P. C. C. and L. S. J. had responsibility for producing the final version of the article.

\section{References}

1. Calder PC, Albers R, Antoine JM, et al. (2009) Inflammatory disease processes and interactions with nutrition. Br J Nutr 101, Suppl. 1, S1-45.

2. Kolb H \& Mandrup-Poulsen T (2010) The global diabetes epidemic as a consequence of lifestyle-induced low-grade inflammation. Diabetologia 53, 10-20.

3. Fernandez-Real JM \& Pickup JC (2008) Innate immunity, insulin resistance and type 2 diabetes. Trends Endocrinol Metab 19, 10-16.

4. Herder C, Peltonen M, Koenig W, et al. (2006) Systemic immune mediators and lifestyle changes in the prevention of type 2 diabetes: results from the Finnish Diabetes Prevention Study. Diabetes 55, 2340-2346.

5. Herder C, Illig T, Rathmann W, et al. (2005) Inflammation and type 2 diabetes: results from KORA Augsburg. Gesundheitswesen 67, Suppl. 1, S115-S121.

6. Muller S, Martin S, Koenig W, et al. (2002) Impaired glucose tolerance is associated with increased serum concentrations of interleukin 6 and co-regulated acute-phase proteins but not TNF-alpha or its receptors. Diabetologia 45, 805-812.

7. Kim CS, Park HS, Kawada T, et al. (2006) Circulating levels of MCP- 1 and IL- 8 are elevated in human obese subjects and associated with obesity-related parameters. Int $J$ Obes (Lond) 30, 1347-1355.

8. Hotamisligil GS, Shargill NS \& Spiegelman BM (1993) Adipose expression of tumor necrosis factor-alpha: direct role in obesity-linked insulin resistance. Science 259, 87-91.

9. Trayhurn P \& Wood IS (2004) Adipokines: inflammation and the pleiotropic role of white adipose tissue. Br J Nutr 92, 347-355.

10. Tilg H \& Moschen AR (2006) Adipocytokines: mediators linking adipose tissue, inflammation and immunity. Nat Rev Immunol 6, 772-783.

11. Wellen KE \& Hotamisligil GS (2003) Obesity-induced inflammatory changes in adipose tissue. J Clin Invest 112, $1785-1788$.

12. Koerner A, Kratzsch J \& Kiess W (2005) Adipocytokines: leptin-the classical, resistin-the controversical, adiponectin-the promising, and more to come. Best Pract Res Clin Endocrinol Metab 19, 525-546.

13. Trujillo ME \& Scherer PE (2006) Adipose tissue-derived factors: impact on health and disease. Endocr Rev 27, $762-778$.

14. Stofkova A (2009) Leptin and adiponectin: from energy and metabolic dysbalance to inflammation and autoimmunity. Endocr Regul 43, 157-168.

15. Gil-Campos M, Canete RR \& Gil A (2004) Adiponectin, the missing link in insulin resistance and obesity. Clin Nutr 23, 963-974.

16. Fukuhara A, Matsuda M, Nishizawa M, et al. (2005) Visfatin: a protein secreted by visceral fat that mimics the effects of insulin. Science 307, 426-430.

17. McTernan PG, Kusminski CM \& Kumar S (2006) Resistin. Curr Opin Lipidol 17, 170-175.
18. Yang RZ, Lee $\mathrm{MJ}$, Hu H, et al. (2006) Identification of omentin as a novel depot-specific adipokine in human adipose tissue: possible role in modulating insulin action. Am J Physiol Endocrinol Metab 290, E1253-E1261.

19. Yang RZ, Lee MJ, Hu H, et al. (2006) Acute-phase serum amyloid A: an inflammatory adipokine and potential link between obesity and its metabolic complications. PLOS Med 3, e287.

20. Sethi JK \& Vidal-Puig A (2005) Visfatin: the missing link between intra-abdominal obesity and diabetes? Trends Mol Med 11, 344-347.

21. Stephens JM \& Vidal-Puig AJ (2006) An update on visfatin/ pre-B cell colony-enhancing factor, an ubiquitously expressed, illusive cytokine that is regulated in obesity. Curr Opin Lipidol 17, 128-131.

22. Juhan-Vague I, Alessi MC, Mavri A, et al. (2003) Plasminogen activator inhibitor-1, inflammation, obesity, insulin resistance and vascular risk. J Thromb Haemost 1, $1575-1579$

23. Poitou C, Viguerie N, Cancello R, et al. (2005) Serum amyloid A: production by human white adipocyte and regulation by obesity and nutrition. Diabetologia 48, 519-528.

24. Somm E, Cettour-Rose P, Asensio C, et al. (2006) Interleukin-1 receptor antagonist is upregulated during dietinduced obesity and regulates insulin sensitivity in rodents. Diabetologia 49, 387-393.

25. Somm E, Henrichot E, Pernin A, et al. (2005) Decreased fat mass in interleukin-1 receptor antagonist-deficient mice: impact on adipogenesis, food intake, and energy expenditure. Diabetes 54, 3503-3509.

26. Meier CA, Bobbioni E, Gabay C, et al. (2002) IL-1 receptor antagonist serum levels are increased in human obesity: a possible link to the resistance to leptin? J Clin Endocrinol Metab 87, 1184-1188.

27. Netea MG, Joosten LA, Lewis E, et al. (2006) Deficiency of interleukin-18 in mice leads to hyperphagia, obesity and insulin resistance. Nat Med 12, 650-656.

28. Skurk T, Kolb H, Muller-Scholze S, et al. (2005) The proatherogenic cytokine interleukin-18 is secreted by human adipocytes. Eur J Endocrinol 152, 863-868.

29. Skurk T, berti-Huber C, Herder C, et al. (2007) Relationship between adipocyte size and adipokine expression and secretion. J Clin Endocrinol Metab 92, 1023-1033.

30. Straczkowski M, Kowalska I, Nikolajuk A, et al. (2007) Increased serum interleukin-18 concentration is associated with hypoadiponectinemia in obesity, independently of insulin resistance. Int J Obes (Lond) 31, 221-225.

31. Cancello R, Henegar C, Viguerie N, et al. (2005) Reduction of macrophage infiltration and chemoattractant gene expression changes in white adipose tissue of morbidly obese subjects after surgery-induced weight loss. Diabetes 54, 2277-2286.

32. Juge-Aubry CE, Somm E, Pernin A, et al. (2005) Adipose tissue is a regulated source of interleukin-10. Cytokine $\mathbf{2 9}$, $270-274$.

33. Skurk T, Herder C, Kraft I, et al. (2005) Production and release of macrophage migration inhibitory factor from human adipocytes. Endocrinology 146, 1006-1011.

34. Straczkowski M, Dzienis-Straczkowska S, Stepien A, et al. (2002) Plasma interleukin- 8 concentrations are increased in obese subjects and related to fat mass and tumor necrosis factor-alpha system. J Clin Endocrinol Metab 87, 4602-4606.

35. Straczkowski M, Kowalska I, Nikolajuk A, et al. (2003) Plasma interleukin 8 concentrations in obese subjects with impaired glucose tolerance. Cardiovasc Diabetol 2, 5 . 
36. Curat CA, Wegner V, Sengenes C, et al. (2006) Macrophages in human visceral adipose tissue: increased accumulation in obesity and a source of resistin and visfatin. Diabetologia 49, 744-747.

37. Herder C, Hauner H, Kempf K, et al. (2007) Constitutive and regulated expression and secretion of interferongamma-inducible protein 10 (IP-10/CXCL10) in human adipocytes. Int J Obes (Lond) 31, 403-410.

38. Fain JN, Tichansky DS \& Madan AK (2005) Transforming growth factor beta1 release by human adipose tissue is enhanced in obesity. Metabolism 54, 1546-1551.

39. Gabrielsson BG, Johansson JM, Lonn M, et al. (2003) High expression of complement components in omental adipose tissue in obese men. Obes Res 11, 699-708.

40. Guerre-Millo M (2006) Adipose tissue secretory function: implication in metabolic and cardiovascular complications of obesity. J Soc Biol 200, 37-43.

41. Graham TE, Yang Q, Bluher M, et al. (2006) Retinol-binding protein 4 and insulin resistance in lean, obese, and diabetic subjects. N Engl J Med 354, 2552-2563.

42. Yang Q, Graham TE, Mody N, et al. (2005) Serum retinol binding protein 4 contributes to insulin resistance in obesity and type 2 diabetes. Nature 436, 356-362.

43. Janke J, Engeli S, Boschmann M, et al. (2006) Retinol-binding protein 4 in human obesity. Diabetes 55, 2805-2810.

44. Carey VJ, Walters EE, Colditz GA, et al. (1997) Body fat distribution and risk of non-insulin-dependent diabetes mellitus in women. The Nurses' Health Study. Am J Epidemiol 145, 614-619.

45. Kissebah AH, Vydelingum N, Murray R, et al. (1982) Relation of body fat distribution to metabolic complications of obesity. J Clin Endocrinol Metab 54, 254-260.

46. Visser M, Bouter LM, McQuillan GM, et al. (1999) Elevated C-reactive protein levels in overweight and obese adults. JAMA 282, 2131-2135.

47. Cook DG, Mendall MA, Whincup PH, et al. (2000) C-reactive protein concentration in children: relationship to adiposity and other cardiovascular risk factors. Atherosclerosis 149, 139-150

48. Yudkin JS (2003) Adipose tissue, insulin action and vascular disease: inflammatory signals. Int J Obes Relat Metab Disord 27, Suppl. 3, S25-S28.

49. Wellen KE \& Hotamisligil GS (2005) Inflammation, stress, and diabetes. J Clin Invest 115, 1111-1119.

50. Despres JP \& Lemieux I (2006) Abdominal obesity and metabolic syndrome. Nature 444, 881-887.

51. Lafontan M \& Girard J (2008) Impact of visceral adipose tissue on liver metabolism. Part I: heterogeneity of adipose tissue and functional properties of visceral adipose tissue. Diabetes Metab 34, 317-327.

52. van Harmelen V, Dicker A, Ryden M, et al. (2002) Increased lipolysis and decreased leptin production by human omental as compared with subcutaneous preadipocytes. Diabetes 51, 2029-2036.

53. Alessi MC, Bastelica D, Morange P, et al. (2000) Plasminogen activator inhibitor 1, transforming growth factorbeta1, and BMI are closely associated in human adipose tissue during morbid obesity. Diabetes 49, 1374-1380.

54. Bruun JM, Lihn AS, Madan AK, et al. (2004) Higher production of IL-8 in visceral vs. subcutaneous adipose tissue. Implication of nonadipose cells in adipose tissue. Am J Physiol Endocrinol Metab 286, E8-E13.

55. Bastelica D, Morange P, Berthet B, et al. (2002) Stromal cells are the main plasminogen activator inhibitor-1-producing cells in human fat: evidence of differences between visceral and subcutaneous deposits. Arterioscler Thromb Vasc Biol 22, 173-178.

56. Fain JN, Madan AK, Hiler ML, et al. (2004) Comparison of the release of adipokines by adipose tissue, adipose tissue matrix, and adipocytes from visceral and subcutaneous abdominal adipose tissues of obese humans. Endocrinology 145, 2273-2282.

57. Hauner H (2005) Secretory factors from human adipose tissue and their functional role. Proc Nutr Soc 64, 163-169.

58. de Souza Batista CM, Yang RZ, Lee MJ, et al. (2007) Omentin plasma levels and gene expression are decreased in obesity. Diabetes 56, 1655-1661.

59. Arner P (2006) Visfatin-a true or false trail to type 2 diabetes mellitus. J Clin Endocrinol Metab 91, 28-30.

60. Rabkin SW (2007) Epicardial fat: properties, function and relationship to obesity. Obes Rev 8, 253-261.

61. Silaghi A, Piercecchi-Marti MD, Grino M, et al. (2008) Epicardial adipose tissue extent: relationship with age, body fat distribution, and coronaropathy. Obesity (Silver Spring) 16, 2424-2430.

62. Jernas M, Palming J, Sjoholm K, et al. (2006) Separation of human adipocytes by size: hypertrophic fat cells display distinct gene expression. FASEB J 20, 1540-1542.

63. Weyer C, Foley JE, Bogardus C, et al. (2000) Enlarged subcutaneous abdominal adipocyte size, but not obesity itself, predicts type II diabetes independent of insulin resistance. Diabetologia 43, 1498-1506.

64. Fain JN (2006) Release of interleukins and other inflammatory cytokines by human adipose tissue is enhanced in obesity and primarily due to the nonfat cells. Vitam Horm 74, 443-477.

65. Cancello R, Tordjman J, Poitou C, et al. (2006) Increased infiltration of macrophages in omental adipose tissue is associated with marked hepatic lesions in morbid human obesity. Diabetes 55, 1554-1561.

66. Weisberg SP, McCann D, Desai M, et al. (2003) Obesity is associated with macrophage accumulation in adipose tissue. J Clin Invest 112, 1796-1808.

67. Xu H, Barnes GT, Yang Q, et al. (2003) Chronic inflammation in fat plays a crucial role in the development of obesity-related insulin resistance. J Clin Invest 112, 1821-1830.

68. Henegar C, Tordjman J, Achard V, et al. (2008) Adipose tissue transcriptomic signature highlights the pathological relevance of extracellular matrix in human obesity. Genome Biol 9, R14.

69. Kintscher U, Hartge M, Hess K, et al. (2008) T-lymphocyte infiltration in visceral adipose tissue: a primary event in adipose tissue inflammation and the development of obesity-mediated insulin resistance. Arterioscler Thromb Vasc Biol 28, 1304-1310.

70. Rausch ME, Weisberg S, Vardhana P, et al. (2008) Obesity in $\mathrm{C} 57 \mathrm{BL} / 6 \mathrm{~J}$ mice is characterized by adipose tissue hypoxia and cytotoxic T-cell infiltration. Int $J$ Obes (Lond) $\mathbf{3 2}$, 451-463.

71. Wu H, Ghosh S, Perrard XD, et al. (2007) T-cell accumulation and regulated on activation, normal $\mathrm{T}$ cell expressed and secreted upregulation in adipose tissue in obesity. Circulation 115, 1029-1038.

72. Bornstein SR, Abu-Asab M, Glasow A, et al. (2000) Immunohistochemical and ultrastructural localization of leptin and leptin receptor in human white adipose tissue and differentiating human adipose cells in primary culture. Diabetes 49, 532-538.

73. Cousin B, Munoz O, Andre M, et al. (1999) A role for preadipocytes as macrophage-like cells. FASEB J 13, 305-312. 
74. Cousin B, Andre M, Casteilla L, et al. (2001) Altered macrophage-like functions of preadipocytes in inflammation and genetic obesity. J Cell Physiol 186, 380-386.

75. Lumeng CN, Bodzin JL \& Saltiel AR (2007) Obesity induces a phenotypic switch in adipose tissue macrophage polarization. J Clin Invest 117, 175-184.

76. Olefsky JM \& Glass CK (2010) Macrophages, inflammation, and insulin resistance. Annu Rev Physiol 72, 219-246.

77. Karastergiou K \& Mohamed-Ali V (2010) The autocrine and paracrine roles of adipokines. Mol Cell Endocrinol 318, 69-78.

78. Curat CA, Miranville A, Sengenes C, et al. (2004) From blood monocytes to adipose tissue-resident macrophages: induction of diapedesis by human mature adipocytes. Diabetes 53, 1285-1292.

79. Guzik TJ, Mangalat D \& Korbut R (2006) Adipocytokines novel link between inflammation and vascular function? J Physiol Pharmacol 57, 505-528.

80. Weisberg SP, Hunter D, Huber R, et al. (2006) CCR2 modulates inflammatory and metabolic effects of high-fat feeding. J Clin Invest 116, 115-124.

81. Inouye KE, Shi H, Howard JK, et al. (2007) Absence of CC chemokine ligand 2 does not limit obesity-associated infiltration of macrophages into adipose tissue. Diabetes 56, 2242-2250.

82. Trayhurn P, Wang B \& Wood IS (2008) Hypoxia in adipose tissue: a basis for the dysregulation of tissue function in obesity? Br J Nutr 100, 227-235.

83. Guerre-Millo M, Grosfeld A \& Issad T (2002) Leptin is a hypoxia-inducible gene. Obes Res 10, 856-858.

84. Cinti S, Mitchell G, Barbatelli G, et al. (2005) Adipocyte death defines macrophage localization and function in adipose tissue of obese mice and humans. J Lipid Res $\mathbf{4 6}$, 2347-2355.

85. Bouloumie A, Casteilla L \& Lafontan M (2008) Adipose tissue lymphocytes and macrophages in obesity and insulin resistance: makers or markers, and which comes first? Arterioscler Thromb Vasc Biol 28, 1211-1213.

86. Lagathu C, Bastard JP, Auclair M, et al. (2003) Chronic interleukin-6 (IL-6) treatment increased IL-6 secretion and induced insulin resistance in adipocyte: prevention by rosiglitazone. Biochem Biophys Res Commun 311, 372-379.

87. Lacasa D, Taleb S, Keophiphath M, et al. (2007) Macrophage-secreted factors impair human adipogenesis: involvement of proinflammatory state in preadipocytes. Endocrinology 148, 868-877.

88. Constant VA, Gagnon A, Landry A, et al. (2006) Macrophage-conditioned medium inhibits the differentiation of 3T3-L1 and human abdominal preadipocytes. Diabetologia 49, 1402-1411.

89. Permana PA, Menge C \& Reaven PD (2006) Macrophagesecreted factors induce adipocyte inflammation and insulin resistance. Biochem Biophys Res Commun 341, 507-514.

90. Suganami T, Nishida J \& Ogawa Y (2005) A paracrine loop between adipocytes and macrophages aggravates inflammatory changes: role of free fatty acids and tumor necrosis factor alpha. Arterioscler Thromb Vasc Biol 25, 2062-2068.

91. Kumar A, Takada Y, Boriek AM, et al. (2004) Nuclear factorkappaB: its role in health and disease. J Mol Med 82, 434-448.

92. Perkins ND (2007) Integrating cell-signalling pathways with NF-kappaB and IKK function. Nat Rev Mol Cell Biol 8, 49-62.

93. Sigal LH (2006) Basic science for the clinician 39: NFkappaB-function, activation, control, and consequences. J Clin Rheumatol 12, 207-211.
94. Maury E \& Brichard SM (2010) Adipokine dysregulation, adipose tissue inflammation and metabolic syndrome. Mol Cell Endocrinol 314, 1-16.

95. Suganami T, Tanimoto-Koyama K, Nishida J, et al. (2007) Role of the Toll-like receptor 4/NF-kappaB pathway in saturated fatty acid-induced inflammatory changes in the interaction between adipocytes and macrophages. Arterioscler Thromb Vasc Biol 27, 84-91.

96. Lin Y, Lee H, Berg AH, et al. (2000) The lipopolysaccharideactivated toll-like receptor (TLR)- 4 induces synthesis of the closely related receptor TLR-2 in adipocytes. $J$ Biol Chem 275, 24255-24263.

97. Shi H, Kokoeva MV, Inouye K, et al. (2006) TLR4 links innate immunity and fatty acid-induced insulin resistance. J Clin Invest 116, 3015-3025.

98. Matsuzawa Y (2006) The metabolic syndrome and adipocytokines. FEBS Lett 580, 2917-2921.

99. Matsuzawa Y (2006) Therapy insight: adipocytokines in metabolic syndrome and related cardiovascular disease. Nat Clin Pract Cardiovasc Med 3, 35-42.

100. Chen H (2006) Cellular inflammatory responses: novel insights for obesity and insulin resistance. Pharmacol Res 53, 469-477.

101. Malavazos AE, Cereda E, Morricone L, et al. (2005) Monocyte chemoattractant protein 1: a possible link between visceral adipose tissue-associated inflammation and subclinical echocardiographic abnormalities in uncomplicated obesity. Eur J Endocrinol 153, 871-877.

102. Malavazos AE, Corsi MM, Ermetici F, et al. (2007) Proinflammatory cytokines and cardiac abnormalities in uncomplicated obesity: relationship with abdominal fat deposition. Nutr Metab Cardiovasc Dis 17, 294-302.

103. Ouchi N, Kihara S, Funahashi T, et al. (2003) Obesity, adiponectin and vascular inflammatory disease. Curr Opin Lipidol 14, 561-566.

104. Herder C, Haastert B, Muller-Scholze S, et al. (2005) Association of systemic chemokine concentrations with impaired glucose tolerance and type 2 diabetes: results from the Cooperative Health Research in the Region of Augsburg Survey S4 (KORA S4). Diabetes 54, Suppl. 2, S11-S17.

105. Stentz FB, Umpierrez GE, Cuervo R, et al. (2004) Proinflammatory cytokines, markers of cardiovascular risks, oxidative stress, and lipid peroxidation in patients with hyperglycemic crises. Diabetes 53, 2079-2086.

106. de Luca C \& Olefsky JM (2006) Stressed out about obesity and insulin resistance. Nat Med 12, 41-42.

107. Harman-Boehm I, Bluher M, Redel H, et al. (2007) Macrophage infiltration into omental versus subcutaneous fat across different populations: effect of regional adiposity and the comorbidities of obesity. J Clin Endocrinol Metab 92, 2240-2247.

108. Berg AH \& Scherer PE (2005) Adipose tissue, inflammation, and cardiovascular disease. Circ Res 96, 939-949.

109. Kopelman P (2007) Health risks associated with overweight and obesity. Obes Rev 8, Suppl. 1, 13-17.

110. Ziccardi P, Nappo F, Giugliano G, et al. (2002) Reduction of inflammatory cytokine concentrations and improvement of endothelial functions in obese women after weight loss over one year. Circulation 105, 804-809.

111. Esposito K, Pontillo A, Di Palo C, et al. (2003) Effect of weight loss and lifestyle changes on vascular inflammatory markers in obese women: a randomized trial. JAMA $\mathbf{2 8 9}$, 1799-1804.

112. Heilbronn LK \& Clifton PM (2002) C-reactive protein and coronary artery disease: influence of obesity, caloric restriction and weight loss. J Nutr Biochem 13, 316-321. 
113. Bastard JP, Jardel C, Bruckert E, et al. (2000) Elevated levels of interleukin 6 are reduced in serum and subcutaneous adipose tissue of obese women after weight loss. J Clin Endocrinol Metab 85, 3338-3342.

114. Esposito K, Pontillo A, Ciotola M, et al. (2002) Weight loss reduces interleukin-18 levels in obese women. J Clin Endocrinol Metab 87, 3864-3866.

115. Bastard JP, Vidal H, Jardel C, et al. (2000) Subcutaneous adipose tissue expression of plasminogen activator inhibitor-1 gene during very low calorie diet in obese subjects. Int $J$ Obes Relat Metab Disord 24, 70-74.

116. Gomez-Ambrosi J, Salvador J, Rotellar F, et al. (2006) Increased serum amyloid A concentrations in morbid obesity decrease after gastric bypass. Obes Surg 16, 262-269.

117. Taleb S, Cancello R, Poitou C, et al. (2006) Weight loss reduces adipose tissue cathepsin $\mathrm{S}$ and its circulating levels in morbidly obese women. J Clin Endocrinol Metab 91, 1042-1047.

118. Laimer M, Kaser S, Kranebitter M, et al. (2005) Effect of pronounced weight loss on the nontraditional cardiovascular risk marker matrix metalloproteinase-9 in middle-aged morbidly obese women. Int J Obes (Lond) 29, 498-501.

119. Kopp CW, Kopp HP, Steiner S, et al. (2003) Weight loss reduces tissue factor in morbidly obese patients. Obes Res 11, 950-956.

120. Church TS, Willis MS, Priest EL, et al. (2005) Obesity, macrophage migration inhibitory factor, and weight loss. Int J Obes (Lond) 29, 675-681.

121. Christiansen T, Richelsen B \& Bruun JM (2005) Monocyte chemoattractant protein-1 is produced in isolated adipocytes, associated with adiposity and reduced after weight loss in morbid obese subjects. Int I Obes (Lond) 29 , $146-150$

122. Vasudevan AR, Wu H, Xydakis AM, et al. (2006) Eotaxin and obesity. J Clin Endocrinol Metab 91, 256-261.

123. Krzyzanowska K, Mittermayer F, Krugluger W, et al. (2006) Increase in visfatin after weight loss induced by gastroplastic surgery. Obesity (Silver Spring) 14, 1886-1889.

124. Bastard JP, Hainque B, Dusserre E, et al. (1999) Peroxisome proliferator activated receptor-gamma, leptin and tumor necrosis factor-alpha mRNA expression during very low calorie diet in subcutaneous adipose tissue in obese women. Diabetes Metab Res Rev 15, 92-98.

125. Kopp HP, Kopp CW, Festa A, et al. (2003) Impact of weight loss on inflammatory proteins and their association with the insulin resistance syndrome in morbidly obese patients Arterioscler Thromb Vasc Biol 23, 1042-1047.

126. Poitou C, Coussieu C, Rouault C, et al. (2006) Serum amyloid A: a marker of adiposity-induced low-grade inflammation but not of metabolic status. Obesity (Silver Spring) 14, 309-318.

127. Clement K, Viguerie N, Poitou C, et al. (2004) Weight loss regulates inflammation-related genes in white adipose tissue of obese subjects. FASEB J 18, 1657-1669.

128. de Luca C \& Olefsky JM (2008) Inflammation and insulin resistance. FEBS Lett 582, 97-105.

129. Li L, Naples M, Song H, et al. (2007) LCAT-null mice develop improved hepatic insulin sensitivity through altered regulation of transcription factors and suppressors of cytokine signaling. Am J Physiol Endocrinol Metab 293, E587-E594.

130. Rui HL, Fan E, Zhou HM, et al. (2002) SUMO-1 modification of the C-terminal KVEKVD of Axin is required for JNK activation but has no effect on Wnt signaling. $J$ Biol Chem 277, 42981-42986.
131. Ueki K, Kondo T \& Kahn CR (2004) Suppressor of cytokine signaling 1 (SOCS-1) and SOCS-3 cause insulin resistance through inhibition of tyrosine phosphorylation of insulin receptor substrate proteins by discrete mechanisms. Mol Cell Biol 24, 5434-5446.

132. Kim JH, Kim JE, Liu HY, et al. (2008) Regulation of interleukin-6-induced hepatic insulin resistance by mammalian target of rapamycin through the STAT3-SOCS3 pathway. J Biol Chem 283, 708-715.

133. Lang CH, Dobrescu C \& Bagby GJ (1992) Tumor necrosis factor impairs insulin action on peripheral glucose disposal and hepatic glucose output. Endocrinology 130, 43-52.

134. Miles PD, Romeo OM, Higo K, et al. (1997) TNF-alphainduced insulin resistance in vivo and its prevention by troglitazone. Diabetes 46, 1678-1683.

135. Atsumi T, Cho YR, Leng L, et al. (2007) The proinflammatory cytokine macrophage migration inhibitory factor regulates glucose metabolism during systemic inflammation. J Immunol 179, 5399-5406.

136. Houstis N, Rosen ED \& Lander ES (2006) Reactive oxygen species have a causal role in multiple forms of insulin resistance. Nature 440, 944-948.

137. Nishikawa T, Kukidome D, Sonoda K, et al. (2007) Impact of mitochondrial ROS production in the pathogenesis of insulin resistance. Diabetes Res Clin Pract 77, Suppl. 1, S161-S164.

138. Li Y, Jiang C, Xu G, et al. (2008) Homocysteine upregulates resistin production from adipocytes in vivo and in vitro. Diabetes 57, 817-827.

139. Arner P (2005) Insulin resistance in type 2 diabetes - role of the adipokines. Curr Mol Med 5, 333-339.

140. Yan SF, Barile GR, D'Agati V, et al. (2007) The biology of RAGE and its ligands: uncovering mechanisms at the heart of diabetes and its complications. Curr Diab Rep 7, 146-153.

141. Nomiyama T, Perez-Tilve D, Ogawa D, et al. (2007) Osteopontin mediates obesity-induced adipose tissue macrophage infiltration and insulin resistance in mice. J Clin Invest 117, 2877-2888

142. Clement K \& Langin D (2007) Regulation of inflammationrelated genes in human adipose tissue. J Intern Med $\mathbf{2 6 2}$, 422-430.

143. Kim JK, Kim YJ, Fillmore JJ, et al. (2001) Prevention of fat-induced insulin resistance by salicylate. J Clin Invest 108, $437-446$

144. Yin MJ, Yamamoto Y \& Gaynor RB (1998) The anti-inflammatory agents aspirin and salicylate inhibit the activity of I(kappa)B kinase-beta. Nature 396, 77-80.

145. Shoelson SE, Lee J \& Yuan M (2003) Inflammation and the IKK beta/I kappa B/NF-kappa B axis in obesity- and diet-induced insulin resistance. Int $J$ Obes Relat Metab Disord 27, Suppl. 3, S49-S52.

146. Fleischman A, Shoelson SE, Bernier R, et al. (2008) Salsalate improves glycemia and inflammatory parameters in obese young adults. Diabetes Care 31, 289-294.

147. Wasko MC, Hubert HB, Lingala VB, et al. (2007) Hydroxychloroquine and risk of diabetes in patients with rheumatoid arthritis. JAMA 298, 187-193.

148. Larsen CM, Faulenbach M, Vaag A, et al. (2007) Interleukin1-receptor antagonist in type 2 diabetes mellitus. $N$ Engl J Med 356, 1517-1526.

149. Barbuio R, Milanski M, Bertolo MB, et al. (2007) Infliximab reverses steatosis and improves insulin signal transduction in liver of rats fed a high-fat diet. J Endocrinol 194, $539-550$ 
150. Dominguez H, Storgaard H, Rask-Madsen C, et al. (2005) Metabolic and vascular effects of tumor necrosis factoralpha blockade with etanercept in obese patients with type 2 diabetes. $J$ Vasc Res 42, 517-525.

151. Tam LS, Tomlinson B, Chu TT, et al. (2007) Impact of TNF inhibition on insulin resistance and lipids levels in patients with rheumatoid arthritis. Clin Rheumatol 26, 1495-1498.

152. Di Rocco P, Manco M, Rosa G, et al. (2004) Lowered tumor necrosis factor receptors, but not increased insulin sensitivity, with infliximab. Obes Res 12, 734-739.

153. Borst SE, Lee Y, Conover CF, et al. (2004) Neutralization of tumor necrosis factor-alpha reverses insulin resistance in skeletal muscle but not adipose tissue. Am J Physiol Endocrinol Metab 287, E934-E938.

154. Hansen K, Sickelmann F, Pietrowsky R, et al. (1997) Systemic immune changes following meal intake in humans. Am J Physiol 273, R548-R553.

155. Plat J, Jellema A, Ramakers J, et al. (2007) Weight loss, but not fish oil consumption, improves fasting and postprandial serum lipids, markers of endothelial function, and inflammatory signatures in moderately obese men. J Nutr $\mathbf{1 3 7}$, 2635-2640.

156. Bell DS, O'Keefe JH \& Jellinger P (2008) Postprandial dysmetabolism: the missing link between diabetes and cardiovascular events? Endocr Pract 14, 112-124.

157. O'Keefe JH, Gheewala NM \& O'Keefe JO (2008) Dietary strategies for improving post-prandial glucose, lipids, inflammation, and cardiovascular health. J Am Coll Cardiol 51, 249-255.

158. Napolitano M \& Bravo E (2005) Lipid metabolism and TNFalpha secretion in response to dietary sterols in human monocyte derived macrophages. Eur J Clin Invest 35, 482-490.

159. van Oostrom AJ, Sijmonsma TP, Verseyden C, et al. (2003) Postprandial recruitment of neutrophils may contribute to endothelial dysfunction. J Lipid Res 44, 576-583.

160. Luu NT, Madden J, Calder PC, et al. (2007) Dietary supplementation with fish oil modifies the ability of human monocytes to induce an inflammatory response. $J$ Nutr 137, 2769-2774.

161. Alipour A, van Oostrom AJ, Izraeljan A, et al. (2008) Leukocyte activation by triglyceride-rich lipoproteins. Arterioscler Thromb Vasc Biol 28, 792-797.

162. Burdge GC \& Calder PC (2005) Plasma cytokine response during the postprandial period: a potential causal process in vascular disease? Br J Nutr 93, 3-9.

163. Manning PJ, Sutherland WH, McGrath MM, et al. (2008) Postprandial cytokine concentrations and meal composition in obese and lean women. Obesity (Silver Spring) 16, 2046-2052.

164. Jellema A, Plat J \& Mensink RP (2004) Weight reduction, but not a moderate intake of fish oil, lowers concentrations of inflammatory markers and PAI-1 antigen in obese men during the fasting and postprandial state. Eur J Clin Invest 34, 766-773.

165. Esposito K, Ciotola M, Sasso FC, et al. (2007) Effect of a single high-fat meal on endothelial function in patients with the metabolic syndrome: role of tumor necrosis factor-alpha. Nutr Metab Cardiovasc Dis 17, 274-279.

166. Nappo F, Esposito K, Cioffi M, et al. (2002) Postprandial endothelial activation in healthy subjects and in type 2 diabetic patients: role of fat and carbohydrate meals. $J \mathrm{Am}$ Coll Cardiol 39, 1145-1150.

167. Ceriello A, Assaloni R, Da RR, et al. (2005) Effect of atorvastatin and irbesartan, alone and in combination, on postprandial endothelial dysfunction, oxidative stress, and inflammation in type 2 diabetic patients. Circulation $\mathbf{1 1 1}$ 2518-2524.

168. Erridge C, Attina T, Spickett CM, et al. (2007) A high-fat meal induces low-grade endotoxemia: evidence of a novel mechanism of postprandial inflammation. Am J Clin Nutr 86, 1286-1292.

169. Ceriello A, Bortolotti N, Motz E, et al. (1998) Mealgenerated oxidative stress in type 2 diabetic patients. Diabetes Care 21, 1529-1533.

170. Wellen KE, Fucho R, Gregor MF, et al. (2007) Coordinated regulation of nutrient and inflammatory responses by STAMP2 is essential for metabolic homeostasis. Cell $\mathbf{1 2 9}$ 537-548.

171. Abedini A \& Shoelson SE (2007) Inflammation and obesity: STAMPing out insulin resistance? Immunol Cell Biol 85, 399-400.

172. Candore G, Colonna-Romano G, Balistreri CR, et al. (2006) Biology of longevity: role of the innate immune system. Rejuvenation Res 9, 143-148.

173. Krabbe KS, Pedersen M \& Bruunsgaard H (2004) Inflammatory mediators in the elderly. Exp Gerontol 39, 687-699.

174. Wei J, Xu H, Davies JL, et al. (1992) Increase of plasma IL-6 concentration with age in healthy subjects. Life Sci $\mathbf{5 1}$ 1953-1956.

175. Hager K, Machein U, Krieger S, et al. (1994) Interleukin-6 and selected plasma proteins in healthy persons of different ages. Neurobiol Aging 15, 771-772.

176. Ferrucci L, Corsi A, Lauretani F, et al. (2005) The origins of age-related proinflammatory state. Blood 105, 2294-2299.

177. Pedersen M, Bruunsgaard H, Weis N, et al. (2003) Circulating levels of TNF-alpha and IL-6-relation to truncal fat mass and muscle mass in healthy elderly individuals and in patients with type-2 diabetes. Mech Ageing Dev 124, 495-502.

178. Paolisso G, Rizzo MR, Mazziotti G, et al. (1998) Advancing age and insulin resistance: role of plasma tumor necrosis factor-alpha. Am J Physiol 275, E294-E299.

179. Bruunsgaard H, Andersen-Ranberg K, Jeune B, et al. (1999) A high plasma concentration of TNF-alpha is associated with dementia in centenarians. $J$ Gerontol A Biol Sci Med Sci 54, M357-M364.

180. Ballou SP, Lozanski FB, Hodder S, et al. (1996) Quantitative and qualitative alterations of acute-phase proteins in healthy elderly persons. Age Ageing 25, 224-230.

181. Roubenoff R, Harris TB, Abad LW, et al. (1998) Monocyte cytokine production in an elderly population: effect of age and inflammation. J Gerontol A Biol Sci Med Sci 53, M20-M26.

182. Franceschi C, Capri M, Monti D, et al. (2007) Inflammaging and anti-inflammaging: a systemic perspective on aging and longevity emerged from studies in humans. Mech Ageing Dev 128, 92-105.

183. Gangemi S, Pescara L, D'Urbano E, et al. (2005) Aging is characterized by a profound reduction in anti-inflammatory lipoxin A4 levels. Exp Gerontol 40, 612-614.

184. Ahluwalia N (2004) Aging, nutrition and immune function. J Nutr Health Aging 8, 2-6.

185. Lesourd B (2006) Nutritional factors and immunological ageing. Proc Nutr Soc 65, 319-325.

186. Jenny NS, Tracy RP, Ogg MS, et al. (2002) In the elderly, interleukin-6 plasma levels and the $-174 \mathrm{G}>\mathrm{C}$ polymorphism are associated with the development of cardiovascular disease. Arterioscler Thromb Vasc Biol 22 , 2066-2071 
187. Bruunsgaard H (2002) Effects of tumor necrosis factor-alpha and interleukin-6 in elderly populations. Eur Cytokine Netw 13, 389-391.

188. Nakhai Pour HR, Grobbee DE, Muller M, et al. (2007) Association of endogenous sex hormone with C-reactive protein levels in middle-aged and elderly men. Clin Endocrinol (Oxf) 66, 394-398.

189. Guigoz Y, Dore J \& Schiffrin EJ (2008) The inflammatory status of old age can be nurtured from the intestinal environment. Curr Opin Clin Nutr Metab Care 11, 13-20.

190. Bruunsgaard H, Ladelund S, Pedersen AN, et al. (2003) Predicting death from tumour necrosis factor-alpha and interleukin-6 in 80-year-old people. Clin Exp Immunol 132, 24-31.

191. Harris TB, Ferrucci L, Tracy RP, et al. (1999) Associations of elevated interleukin-6 and C-reactive protein levels with mortality in the elderly. Am J Med 106, 506-512.

192. Reuben DB, Cheh AI, Harris TB, et al. (2002) Peripheral blood markers of inflammation predict mortality and functional decline in high-functioning community-dwelling older persons. J Am Geriatr Soc 50, 638-644.

193. Volpato S, Guralnik JM, Ferrucci L, et al. (2001) Cardiovascular disease, interleukin-6, and risk of mortality in older women: the women's health and aging study. Circulation 103, 947-953.

194. Bruunsgaard H, Pedersen M \& Pedersen BK (2001) Aging and proinflammatory cytokines. Curr Opin Hematol 8, $131-136$

195. Pate RR, Pratt M, Blair SN, et al. (1995) Physical activity and public health. A recommendation from the Centers for Disease Control and Prevention and the American College of Sports Medicine. JAMA 273, 402-407.

196. Caspersen CJ, Powell KE \& Christenson GM (1985) Physical activity, exercise, and physical fitness: definitions and distinctions for health-related research. Public Health Rep 100, 126-131.

197. Shephard RJ, Allen C, Benade AJ, et al. (1968) The maximum oxygen intake. An international reference standard of cardiorespiratory fitness. Bull World Health Organ 38 , $757-764$

198. Hamer M (2007) The relative influences of fitness and fatness on inflammatory factors. Prev Med 44, 3-11.

199. Petersen AM \& Pedersen BK (2005) The anti-inflammatory effect of exercise. J Appl Physiol 98, 1154-1162.

200. Nicklas BJ, You T \& Pahor M (2005) Behavioural treatments for chronic systemic inflammation: effects of dietary weight loss and exercise training. CMAJ 172, 1199-1209.

201. Kasapis C \& Thompson PD (2005) The effects of physical activity on serum C-reactive protein and inflammatory markers: a systematic review. J Am Coll Cardiol 45, 1563-1569.

202. Panagiotakos DB, Kokkinos P, Manios Y, et al. (2004) Physical activity and markers of inflammation and thrombosis related to coronary heart disease. Prev Cardiol 7, 190-194.

203. Tomaszewski M, Charchar FJ, Przybycin M, et al. (2003) Strikingly low circulating CRP concentrations in ultramarathon runners independent of markers of adiposity: how low can you go? Arterioscler Thromb Vasc Biol 23 , $1640-1644$

204. Northoff H, Weinstock C \& Berg A (1994) The cytokine response to strenuous exercise. Int J Sports Med $\mathbf{1 5}$ Suppl. 3, S167-S171.

205. Wärnberg J, Nova E, Romeo J, et al. (2007) Lifestyle-related determinants of inflammation in adolescence. $\mathrm{Br} J \mathrm{Nutr}$ 98, Suppl. 1, S116-S120.
206. Pedersen BK (2006) The anti-inflammatory effect of exercise: its role in diabetes and cardiovascular disease control. Essays Biochem 42, 105-117.

207. Pedersen BK, Steensberg A, Fischer C, et al. (2003) Searching for the exercise factor: is IL- 6 a candidate? J Muscle Res Cell Motil 24, 113-119.

208. Fischer CP, Berntsen A, Perstrup LB, et al. (2007) Plasma levels of interleukin- 6 and C-reactive protein are associated with physical inactivity independent of obesity. Scand J Med Sci Sports 17, 580-587.

209. Ostrowski K, Rohde T, Zacho M, et al. (1998) Evidence that interleukin-6 is produced in human skeletal muscle during prolonged running. J Physiol 508, Pt 3, 949-953.

210. Fischer CP (2006) Interleukin-6 in acute exercise and training: what is the biological relevance? Exerc Immunol Rev 12, 6-33.

211. Fallon KE, Fallon SK \& Boston T (2001) The acute phase response and exercise: court and field sports. Br J Sports Med 35, 170-173.

212. Mattusch F, Dufaux B, Heine O, et al. (2000) Reduction of the plasma concentration of $\mathrm{C}$-reactive protein following nine months of endurance training. Int J Sports Med $\mathbf{2 1}$, $21-24$.

213. Hammett CJ, Prapavessis H, Baldi JC, et al. (2006) Effects of exercise training on 5 inflammatory markers associated with cardiovascular risk. Am Heart J 151, 367.

214. Hammett CJ, Oxenham HC, Baldi JC, et al. (2004) Effect of six months' exercise training on C-reactive protein levels in healthy elderly subjects. J Am Coll Cardiol 44, 2411-2413.

215. Bautmans I, Njemini R, Vasseur S, et al. (2005) Biochemical changes in response to intensive resistance exercise training in the elderly. Gerontology 51, 253-265.

216. Nicklas BJ, Ambrosius W, Messier SP, et al. (2004) Dietinduced weight loss, exercise, and chronic inflammation in older, obese adults: a randomized controlled clinical trial. Am J Clin Nutr 79, 544-551.

217. Marcell TJ, McAuley KA, Traustadottir T, et al. (2005) Exercise training is not associated with improved levels of C-reactive protein or adiponectin. Metabolism 54, 533-541.

218. Fischer CP, Plomgaard P, Hansen AK, et al. (2004) Endurance training reduces the contraction-induced interleukin-6 mRNA expression in human skeletal muscle. Am J Physiol Endocrinol Metab 287, E1189-E1194.

219. Kohut ML, McCann DA, Russell DW, et al. (2006) Aerobic exercise, but not flexibility/resistance exercise, reduces serum IL-18, CRP, and IL- 6 independent of beta-blockers, BMI, and psychosocial factors in older adults. Brain Behav Immun 20, 201-209.

220. Stewart LK, Flynn MG, Campbell WW, et al. (2005) Influence of exercise training and age on CD14 + cell-surface expression of toll-like receptor 2 and 4. Brain Behav Immun 19, 389-397.

221. Lakka TA, Lakka HM, Rankinen T, et al. (2005) Effect of exercise training on plasma levels of C-reactive protein in healthy adults: the HERITAGE Family Study. Eur Heart $J$ 26, 2018-2025.

222. Pearson TA, Mensah GA, Alexander RW, et al. (2003) Markers of inflammation and cardiovascular disease: application to clinical and public health practice: a statement for healthcare professionals from the Centers for Disease Control and Prevention and the American Heart Association. Circulation 107, 499-511.

223. Elosua R, Bartali B, Ordovas JM, et al. (2005) Association between physical activity, physical performance, and inflammatory biomarkers in an elderly population: the 
InCHIANTI study. I Gerontol A Biol Sci Med Sci 60 , $760-767$.

224. Colbert LH, Visser M, Simonsick EM, et al. (2004) Physical activity, exercise, and inflammatory markers in older adults: findings from the Health, Aging and Body Composition Study. J Am Geriatr Soc 52, 1098-1104.

225. Wannamethee SG, Lowe GD, Whincup PH, et al. (2002) Physical activity and hemostatic and inflammatory variables in elderly men. Circulation 105, 1785-1790.

226. Reuben DB, Judd-Hamilton L, Harris TB, et al. (2003) The associations between physical activity and inflammatory markers in high-functioning older persons: MacArthur Studies of Successful Aging. J Am Geriatr Soc 51, 1125-1130.

227. Geffken DF, Cushman M, Burke GL, et al. (2001) Association between physical activity and markers of inflammation in a healthy elderly population. Am J Epidemiol 153, 242-250.

228. Taaffe DR, Harris TB, Ferrucci L, et al. (2000) Crosssectional and prospective relationships of interleukin-6 and C-reactive protein with physical performance in elderly persons: MacArthur studies of successful aging. J Gerontol A Biol Sci Med Sci 55, M709-M715.

229. Albert MA, Glynn RJ \& Ridker PM (2004) Effect of physical activity on serum C-reactive protein. Am J Cardiol 93, 221-225.

230. Jankord R \& Jemiolo B (2004) Influence of physical activity on serum IL-6 and IL-10 levels in healthy older men. Med Sci Sports Exerc 36, 960-964.

231. Cesari M, Penninx BW, Pahor M, et al. (2004) Inflammatory markers and physical performance in older persons: the InCHIANTI study. J Gerontol A Biol Sci Med Sci 59, 242-248.

232. Visser M, Pahor M, Taaffe DR, et al. (2002) Relationship of interleukin-6 and tumor necrosis factor-alpha with muscle mass and muscle strength in elderly men and women: the Health ABC Study. J Gerontol A Biol Sci Med Sci 57, M326-M332.

233. Smith JK, Dykes R, Douglas JE, et al. (1999) Long-term exercise and atherogenic activity of blood mononuclear cells in persons at risk of developing ischemic heart disease. JAMA 281, 1722-1727.

234. Goldhammer E, Tanchilevitch A, Maor I, et al. (2005) Exercise training modulates cytokines activity in coronary heart disease patients. Int J Cardiol 100, 93-99.

235. Tisi PV, Hulse M, Chulakadabba A, et al. (1997) Exercise training for intermittent claudication: does it adversely affect biochemical markers of the exercise-induced inflammatory response? Eur J Vasc Endovasc Surg 14, 344-350.

236. Milani RV, Lavie CJ \& Mehra MR (2004) Reduction in C-reactive protein through cardiac rehabilitation and exercise training. J Am Coll Cardiol 43, 1056-1061.

237. McGavock JM, Mandic S, Vonder Muhll I, et al. (2004) Low cardiorespiratory fitness is associated with elevated C-reactive protein levels in women with type 2 diabetes. Diabetes Care 27, 320-325.

238. Aronson D, Sella R, Sheikh-Ahmad M, et al. (2004) The association between cardiorespiratory fitness and C-reactive protein in subjects with the metabolic syndrome. $J \mathrm{Am}$ Coll Cardiol 44, 2003-2007.

239. Aronson D, Sheikh-Ahmad M, Avizohar O, et al. (2004) C-Reactive protein is inversely related to physical fitness in middle-aged subjects. Atherosclerosis 176, 173-179.

240. Panagiotakos DB, Pitsavos C, Chrysohoou C, et al. (2005) The associations between leisure-time physical activity and inflammatory and coagulation markers related to cardiovascular disease: the ATTICA Study. Prev Med $\mathbf{4 0}$ 432-437.

241. Abramson JL \& Vaccarino V (2002) Relationship between physical activity and inflammation among apparently healthy middle-aged and older US adults. Arch Intern Med 162, 1286-1292.

242. King DE, Carek P, Mainous AG III, et al. (2003) Inflammatory markers and exercise: differences related to exercise type. Med Sci Sports Exerc 35, 575-581.

243. Pischon T, Hankinson SE, Hotamisligil GS, et al. (2003) Leisure-time physical activity and reduced plasma levels of obesity-related inflammatory markers. Obes Res 11, 1055-1064.

244. Mora S, Lee IM, Buring JE, et al. (2006) Association of physical activity and body mass index with novel and traditional cardiovascular biomarkers in women. JAMA 295, 1412-1419.

245. Rawson ES, Freedson PS, Osganian SK, et al. (2003) Body mass index, but not physical activity, is associated with C-reactive protein. Med Sci Sports Exerc 35, 1160-1166.

246. Verdaet D, Dendale P, De BD, et al. (2004) Association between leisure time physical activity and markers of chronic inflammation related to coronary heart disease. Atherosclerosis 176, 303-310.

247. Raitakari M, Mansikkaniemi K, Marniemi J, et al. (2005) Distribution and determinants of serum high-sensitive C-reactive protein in a population of young adults: The Cardiovascular Risk in Young Finns Study. J Intern Med 258, 428-434

248. Church TS, Barlow CE, Earnest CP, et al. (2002) Associations between cardiorespiratory fitness and C-reactive protein in men. Arterioscler Thromb Vasc Biol 22, 1869-1876.

249. Church TS, Finley CE, Earnest CP, et al. (2002) Relative associations of fitness and fatness to fibrinogen, white blood cell count, uric acid and metabolic syndrome. Int J Obes Relat Metab Disord 26, 805-813.

250. Diaz VA, Player MS, Mainous AG III, et al. (2006) Competing impact of excess weight versus cardiorespiratory fitness on cardiovascular risk. Am J Cardiol 98, 1468-1471.

251. Isasi CR, Deckelbaum RJ, Tracy RP, et al. (2003) Physical fitness and C-reactive protein level in children and young adults: the Columbia University BioMarkers Study. Pediatrics 111, 332-338.

252. Nemet D, Wang P, Funahashi T, et al. (2003) Adipocytokines, body composition, and fitness in children. Pediatr Res 53, 148-152.

253. Halle M, Korsten-Reck U, Wolfarth B, et al. (2004) Lowgrade systemic inflammation in overweight children: impact of physical fitness. Exerc Immunol Rev 10, 66-74.

254. Kelly AS, Wetzsteon RJ, Kaiser DR, et al. (2004) Inflammation, insulin, and endothelial function in overweight children and adolescents: the role of exercise. $J$ Pediatr 145, 731-736.

255. Williams MJ, Milne BJ, Hancox RJ, et al. (2005) C-reactive protein and cardiorespiratory fitness in young adults. Eur J Cardiovasc Prev Rehabil 12, 216-220.

256. Platat C, Wagner A, Klumpp T, et al. (2006) Relationships of physical activity with metabolic syndrome features and low-grade inflammation in adolescents. Diabetologia 49 2078-2085.

257. Ruiz JR, Ortega FB \& Warnberg J (2007) Associations of low-grade inflammation with physical activity, fitness and fatness in prepubertal children; the European Youth Heart Study. Int J Obes (Lond) 31, 1545-1551. 
258. Wärnberg J (2006) Inflammatory status in adolescents; the impact of health determinants such as overweight and and fitness. PhD Thesis, Karolinska Institutet.

259. Thomas NE, Baker JS, Graham MR, et al. (2008) C-reactive protein in schoolchildren and its relation to adiposity, physical activity, aerobic fitness and habitual diet. $\mathrm{Br} J$ Sports Med 42, 357-360.

260. Ruiz JR, Ortega FB, Warnberg J, et al. (2008) Inflammatory proteins and muscle strength in adolescents: the Avena study. Arch Pediatr Adolesc Med 162, 462-468.

261. Dandona P, Aljada A, Chaudhuri A, et al. (2005) Metabolic syndrome: a comprehensive perspective based on interactions between obesity, diabetes, and inflammation. Circulation 111, 1448-1454.

262. Moeller SM, Reedy J, Millen AE, et al. (2007) Dietary patterns: challenges and opportunities in dietary patterns research an Experimental Biology workshop, April 1, 2006. J Am Diet Assoc 107, 1233-1239.

263. Newby PK, Muller D, Hallfrisch J, et al. (2003) Dietary patterns and changes in body mass index and waist circumference in adults. Am J Clin Nutr 77, 1417-1425.

264. Michels KB \& Schulze MB (2005) Can dietary patterns help us detect diet-disease associations? Nutr Res Rev 18, 241-248.

265. Newby PK \& Tucker KL (2004) Empirically derived eating patterns using factor or cluster analysis: a review. Nutr Rev 62, 177-203.

266. Hu FB (2002) Dietary pattern analysis: a new direction in nutritional epidemiology. Curr Opin Lipidol 13, 3-9.

267. Holloszy JO \& Fontana L (2007) Caloric restriction in humans. Exp Gerontol 42, 709-712.

268. Kolb H \& Mandrup-Poulsen T (2005) An immune origin of type 2 diabetes? Diabetologia 48, 1038-1050.

269. Johnson JB, Summer W, Cutler RG, et al. (2007) Alternate day calorie restriction improves clinical findings and reduces markers of oxidative stress and inflammation in overweight adults with moderate asthma. Free Radic Biol Med 42, 665-674

270. Madsen EL, Rissanen A, Bruun JM, et al. (2008) Weight loss larger than $10 \%$ is needed for general improvement of levels of circulating adiponectin and markers of inflammation in obese subjects: a 3-year weight loss study. Eur J Endocrinol 158, 179-187.

271. Guarente L \& Picard F (2005) Calorie restriction-the SIR2 connection. Cell 120, 473-482

272. Dali-Youcef N, Lagouge M, Froelich S, et al. (2007) Sirtuins: the 'magnificent seven', function, metabolism and longevity. Ann Med 39, 335-345.

273. Yamamoto H, Schoonjans K \& Auwerx J (2007) Sirtuin functions in health and disease. Mol Endocrinol 21, 1745-1755.

274. Salminen A, Ojala J, Huuskonen J, et al. (2008) Interaction of aging-associated signaling cascades: inhibition of NF-kappaB signaling by longevity factors FoxOs and SIRT1. Cell Mol Life Sci 65, 1049-1058.

275. Buteau J \& Accili D (2007) Regulation of pancreatic beta-cell function by the forkhead protein FoxO1. Diabetes Obes Metab 9, Suppl. 2, 140-146.

276. Huang H \& Tindall DJ (2007) CDK2 and FOXO1: a fork in the road for cell fate decisions. Cell Cycle 6, 902-906.

277. Kim DH, Kim JY, Yu BP, et al. (2008) The activation of NF-kappaB through Akt-induced FOXO1 phosphorylation during aging and its modulation by calorie restriction Biogerontology 9, 33-47.

278. Nakae J, Oki M \& Cao Y (2008) The FoxO transcription factors and metabolic regulation. FEBS Lett 582, 54-67.
279. Sharman MJ \& Volek JS (2004) Weight loss leads to reductions in inflammatory biomarkers after a verylow-carbohydrate diet and a low-fat diet in overweight men. Clin Sci (Lond) 107, 365-369.

280. Baur JA, Pearson KJ, Price NL, et al. (2006) Resveratrol improves health and survival of mice on a high-calorie diet. Nature 444, 337-342.

281. Baur JA \& Sinclair DA (2006) Therapeutic potential of resveratrol: the in vivo evidence. Nat Rev Drug Discov 5 , 493-506.

282. Dai J, Miller AH, Bremner JD, et al. (2008) Adherence to the Mediterranean diet is inversely associated with circulating interleukin-6 among middle-aged men: a twin study. Circulation 117, 169-175.

283. Chrysohoou C, Panagiotakos DB, Pitsavos C, et al. (2004) Adherence to the Mediterranean diet attenuates inflammation and coagulation process in healthy adults: The ATTICA Study. J Am Coll Cardiol 44, 152-158.

284. Esposito K, Marfella R, Ciotola M, et al. (2004) Effect of a Mediterranean-style diet on endothelial dysfunction and markers of vascular inflammation in the metabolic syndrome: a randomized trial. JAMA 292, 1440-1446.

285. Fung TT, McCullough ML, Newby PK, et al. (2005) Diet-quality scores and plasma concentrations of markers of inflammation and endothelial dysfunction. Am J Clin Nutr 82, 163-173.

286. Trichopoulou A, Costacou T, Bamia C, et al. (2003) Adherence to a Mediterranean diet and survival in a Greek population. N Engl J Med 348, 2599-2608.

287. Pitsavos C, Panagiotakos DB, Tzima N, et al. (2007) Diet, exercise, and C-reactive protein levels in people with abdominal obesity: the ATTICA epidemiological study. Angiology 58, 225-233.

288. Salas-Salvado J, Garcia-Arellano A, Estruch R, et al. (2008) Components of the Mediterranean-type food pattern and serum inflammatory markers among patients at high risk for cardiovascular disease. Eur J Clin Nutr 62, 651-659.

289. Ambring A, Johansson M, Axelsen M, et al. (2006) Mediterranean-inspired diet lowers the ratio of serum phospholipid $n$-6 to $n$-3 fatty acids, the number of leukocytes and platelets, and vascular endothelial growth factor in healthy subjects. Am J Clin Nutr 83, 575-581.

290. Bellido C, Lopez-Miranda J, Perez-Martinez P, et al. (2006) The Mediterranean and CHO diets decrease VCAM-1 and E-selectin expression induced by modified low-density lipoprotein in HUVECs. Nutr Metab Cardiovasc Dis 16, $524-530$

291. Esposito K, Ciotola M, Giugliano F, et al. (2006) Mediterranean diet improves erectile function in subjects with the metabolic syndrome. Int J Impot Res 18, 405-410.

292. Esposito K, Ciotola M, Giugliano F, et al. (2007) Mediterranean diet improves sexual function in women with the metabolic syndrome. Int J Impot Res 19, 486-491.

293. Estruch R, Martinez-Gonzalez MA, Corella D, et al. (2006) Effects of a Mediterranean-style diet on cardiovascular risk factors: a randomized trial. Ann Intern Med 145, 1-11.

294. Krajcovicova-Kudlackova M \& Blazicek P (2005) C-reactive protein and nutrition. Bratisl Lek Listy 106, 345-347.

295. Szeto YT, Kwok TC \& Benzie IF (2004) Effects of a longterm vegetarian diet on biomarkers of antioxidant status and cardiovascular disease risk. Nutrition 20, 863-866.

296. Purschwitz K, Rassoul F, Reuter W, et al. (2001) Soluble leukocyte adhesion molecules in vegetarians of various ages. $Z$ Gerontol Geriatr 34, 476-479. 
297. Kennedy E, Ohls J, Carlon S, et al. (1994) The Healthy Eating Index Final Report. Alexandria, VA: Food and Nutrition Service, US Department of Agriculture.

298. McCullough ML, Feskanich D, Stampfer MJ, et al. (2002) Diet quality and major chronic disease risk in men and women: moving toward improved dietary guidance. Am J Clin Nutr 76, 1261-1271.

299. Haines PS, Siega-Riz AM \& Popkin BM (1999) The Diet Quality Index revised: a measurement instrument for populations. J Am Diet Assoc 99, 697-704.

300. Ford ES, Mokdad AH \& Liu S (2005) Healthy Eating Index and C-reactive protein concentration: findings from the National Health and Nutrition Examination Survey III, 1988-1994. Eur J Clin Nutr 59, 278-283.

301. Fargnoli JL, Fung TT, Olenczuk DM, et al. (2008) Adherence to healthy eating patterns is associated with higher circulating total and high-molecular-weight adiponectin and lower resistin concentrations in women from the Nurses' Health Study. Am J Clin Nutr 88, 1213-1224.

302. Boynton A, Neuhouser ML, Wener MH, et al. (2007) Associations between healthy eating patterns and immune

307. Esmaillzadeh A, Kimiagar M, Mehrabi Y, et al. (2007) Dietary patterns and markers of systemic inflammation among Iranian women. J Nutr 137, 992-998.

308. Nanri A, Yoshida D, Yamaji T, et al. (2008) Dietary patterns and C-reactive protein in Japanese men and women. $A m \mathrm{~J}$ Clin Nutr 87, 1488-1496.

309. Nettleton JA, Matijevic N, Follis JL, et al. (2010) Associations between dietary patterns and flow cytometry-measured biomarkers of inflammation and cellular activation in the Atherosclerosis Risk in Communities (ARIC) Carotid Artery MRI Study. Atherosclerosis 212, 260-267.

310. Jensen MK, Koh-Banerjee P, Franz M, et al. (2006) Whole grains, bran, and germ in relation to homocysteine and markers of glycemic control, lipids, and inflammation 1. Am J Clin Nutr 83, 275-283.

311. Lutsey PL, Jacobs DR Jr, Kori S, et al. (2007) Whole grain intake and its cross-sectional association with obesity, insulin resistance, inflammation, diabetes and subclinical CVD: The MESA Study. Br J Nutr 98, 397-405.

312. Mantzoros CS, Williams CJ, Manson JE, et al. (2006) Adherence to the Mediterranean dietary pattern is positively associated with plasma adiponectin concentrations in diabetic women. Am J Clin Nutr 84, 328-335.

313. Masters RC, Liese AD, Haffner SM, et al. (2010) Whole and refined grain intakes are related to inflammatory protein concentrations in human plasma. J Nutr 140, 587-594.
314. Qi L, van Dam RM, Liu S, et al. (2006) Whole-grain, bran, and cereal fiber intakes and markers of systemic inflammation in diabetic women. Diabetes Care 29, 207-211.

315. Qi L, Rimm E, Liu S, et al. (2005) Dietary glycemic index, glycemic load, cereal fiber, and plasma adiponectin concentration in diabetic men. Diabetes Care 28, 1022-1028.

316. Andersson A, Tengblad S, Karlstrom B, et al. (2007) Wholegrain foods do not affect insulin sensitivity or markers of lipid peroxidation and inflammation in healthy, moderately overweight subjects. J Nutr 137, 1401-1407.

317. Brownlee IA, Moore C, Chatfield M, et al. (2010) Markers of cardiovascular risk are not changed by increased whole-grain intake: the WHOLEheart study, a randomised, controlled dietary intervention. Br J Nutr 104, 125-134.

318. Esposito K, Nappo F, Giugliano F, et al. (2003) Meal modulation of circulating interleukin 18 and adiponectin concentrations in healthy subjects and in patients with type 2 diabetes mellitus. Am J Clin Nutr 78, 1135-1140.

319. Katcher HI, Legro RS, Kunselman AR, et al. (2008) The effects of a whole grain-enriched hypocaloric diet on cardiovascular disease risk factors in men and women with metabolic syndrome. Am J Clin Nutr 87, 79-90.

320. Tighe P, Duthie G, Vaughan N, et al. (2010) Effect of increased consumption of whole-grain foods on blood pressure and other cardiovascular risk markers in healthy middle-aged persons: a randomized controlled trial. $A m \mathrm{~J}$ Clin Nutr 92, 733-740.

321. Mateo AN, Aura AM, Selinheimo E, et al. (2011) Bioprocessing of wheat bran in whole wheat bread increases the bioavailability of phenolic acids in men and exerts antiinflammatory effects ex vivo. J Nutr 141, 137-143.

322. Bhupathiraju SN \& Tucker KL (2011) Greater variety in fruit and vegetable intake is associated with lower inflammation in Puerto Rican adults. Am J Clin Nutr 93, 37-46.

323. Chun OK, Chung SJ, Claycombe KJ, et al. (2008) Serum C-reactive protein concentrations are inversely associated with dietary flavonoid intake in U.S. adults. $J$ Nutr 138, $753-760$

324. Esmaillzadeh A, Kimiagar M, Mehrabi Y, et al. (2006) Fruit and vegetable intakes, C-reactive protein, and the metabolic syndrome. Am J Clin Nutr 84, 1489-1497.

325. Gao X, Bermudez OI \& Tucker KL (2004) Plasma C-reactive protein and homocysteine concentrations are related to frequent fruit and vegetable intake in Hispanic and nonHispanic white elders. J Nutr 134, 913-918.

326. Hermsdorff HH, Zulet MA, Puchau B, et al. (2010) Fruit and vegetable consumption and proinflammatory gene expression from peripheral blood mononuclear cells in young adults: a translational study. Nutr Metab (Lond) 7, 42.

327. Holt EM, Steffen LM, Moran A, et al. (2009) Fruit and vegetable consumption and its relation to markers of inflammation and oxidative stress in adolescents. $J \mathrm{Am}$ Diet Assoc 109, 414-421.

328. Oliveira A, Rodriguez-Artalejo F \& Lopes C (2009) The association of fruits, vegetables, antioxidant vitamins and fibre intake with high-sensitivity C-reactive protein: sex and body mass index interactions. Eur J Clin Nutr 63, $1345-1352$.

329. Wannamethee SG, Lowe GD, Rumley A, et al. (2006) Associations of vitamin $\mathrm{C}$ status, fruit and vegetable intakes, and markers of inflammation and hemostasis. Am J Clin Nutr 83, 567-574.

330. Yannakoulia M, Yiannakouris N, Melistas L, et al. (2008) Dietary factors associated with plasma high molecular 
weight and total adiponectin levels in apparently healthy women. Eur J Endocrinol 159, R5-R10.

331. Basu A, Du M, Leyva MJ, et al. (2010) Blueberries decrease cardiovascular risk factors in obese men and women with metabolic syndrome. J Nutr 140, 1582-1587.

332. Castilla P, Echarri R, Davalos A, et al. (2006) Concentrated red grape juice exerts antioxidant, hypolipidemic, and antiinflammatory effects in both hemodialysis patients and healthy subjects. Am J Clin Nutr 84, 252-262.

333. Curtis PJ, Kroon PA, Hollands WJ, et al. (2009) Cardiovascular disease risk biomarkers and liver and kidney function are not altered in postmenopausal women after ingesting an elderberry extract rich in anthocyanins for 12 weeks. J Nutr 139, 2266-2271.

334. Dalgard C, Nielsen F, Morrow JD, et al. (2009) Supplementation with orange and blackcurrant juice, but not vitamin $\mathrm{E}$, improves inflammatory markers in patients with peripheral arterial disease. Br J Nutr 101, 263-269.

335. Esposito K, Nappo F, Giugliano F, et al. (2003) Effect of dietary antioxidants on postprandial endothelial dysfunction induced by a high-fat meal in healthy subjects. $A m \mathrm{~J}$ Clin Nutr 77, 139-143.

336. Freese R, Vaarala O, Turpeinen AM, et al. (2004) No difference in platelet activation or inflammation markers after diets rich or poor in vegetables, berries and apple in healthy subjects. Eur J Nutr 43, 175-182.

337. Karlsen A, Retterstol L, Laake P, et al. (2007) Anthocyanins inhibit nuclear factor-kappaB activation in monocytes and reduce plasma concentrations of pro-inflammatory mediators in healthy adults. J Nutr 137, 1951-1954.

338. Karlsen A, Paur I, Bohn SK, et al. (2010) Bilberry juice modulates plasma concentration of NF-kappaB related inflammatory markers in subjects at increased risk of CVD. Eur J Nutr 49, 345-355.

339. Kelley DS, Rasooly R, Jacob RA, et al. (2006) Consumption of Bing sweet cherries lowers circulating concentrations of inflammation markers in healthy men and women. $J$ Nutr 136, 981-986.

340. Larmo P, Alin J, Salminen E, et al. (2008) Effects of sea buckthorn berries on infections and inflammation: a double-blind, randomized, placebo-controlled trial. Eur $J$ Clin Nutr 62, 1123-1130.

341. Morand C, Dubray C, Milenkovic D, et al. (2011) Hesperidin contributes to the vascular protective effects of orange juice: a randomized crossover study in healthy volunteers. Am J Clin Nutr 93, 73-80.

342. Sanchez-Moreno C, Cano MP, de AB, et al. (2004) Consumption of high-pressurized vegetable soup increases plasma vitamin $\mathrm{C}$ and decreases oxidative stress and inflammatory biomarkers in healthy humans. J Nutr 134, 3021-3025.

343. Sanchez-Moreno C, Cano MP, de AB, et al. (2003) Highpressurized orange juice consumption affects plasma vitamin $\mathrm{C}$, antioxidative status and inflammatory markers in healthy humans. J Nutr 133, 2204-2209.

344. van Doorn MB, Espirito Santo SM, Meijer P, et al. (2006) Effect of garlic powder on C-reactive protein and plasma lipids in overweight and smoking subjects. Am J Clin Nutr 84, 1324-1329.

345. Watzl B, Kulling SE, Moseneder J, et al. (2005) A 4-wk intervention with high intake of carotenoid-rich vegetables and fruit reduces plasma C-reactive protein in healthy, nonsmoking men. Am J Clin Nutr 82, 1052-1058.

346. Zern TL, Wood RJ, Greene C, et al. (2005) Grape polyphenols exert a cardioprotective effect in pre- and postmenopausal women by lowering plasma lipids and reducing oxidative stress. J Nutr 135, 1911-1917.

347. Azadbakht L, Kimiagar M, Mehrabi Y, et al. (2007) Soy consumption, markers of inflammation, and endothelial function: a cross-over study in postmenopausal women with the metabolic syndrome. Diabetes Care 30, 967-973.

348. Azadbakht L, Atabak S \& Esmaillzadeh A (2008) Soy protein intake, cardiorenal indices, and C-reactive protein in type 2 diabetes with nephropathy: a longitudinal randomized clinical trial. Diabetes Care 31, 648-654.

349. Beavers KM, Serra MC, Beavers DP, et al. (2010) Soy and the exercise-induced inflammatory response in postmenopausal women. Appl Physiol Nutr Metab 35, 261-269.

350. Beavers KM, Serra MC, Beavers DP, et al. (2009) Soymilk supplementation does not alter plasma markers of inflammation and oxidative stress in postmenopausal women. Nutr Res 29, 616-622.

351. Blum A, Lang N, Peleg A, et al. (2003) Effects of oral soy protein on markers of inflammation in postmenopausal women with mild hypercholesterolemia. Am Heart $J$ 145, e7.

352. Charles C, Yuskavage J, Carlson O, et al. (2009) Effects of high-dose isoflavones on metabolic and inflammatory markers in healthy postmenopausal women. Menopause 16, 395-400.

353. Greany KA, Nettleton JA, Wangen KE, et al. (2008) Consumption of isoflavone-rich soy protein does not alter homocysteine or markers of inflammation in postmenopausal women. Eur J Clin Nutr 62, 1419-1425.

354. Hanson LN, Engelman HM, Alekel DL, et al. (2006) Effects of soy isoflavones and phytate on homocysteine, C-reactive protein, and iron status in postmenopausal women. $A m \mathrm{~J}$ Clin Nutr 84, 774-780.

355. Hilpert KF, Kris-Etherton PM \& West SG (2005) Lipid response to a low-fat diet with or without soy is modified by C-reactive protein status in moderately hypercholesterolemic adults. J Nutr 135, 1075-1079.

356. Jenkins DJ, Kendall CW, Jackson CJ, et al. (2002) Effects of high- and low-isoflavone soyfoods on blood lipids, oxidized LDL, homocysteine, and blood pressure in hyperlipidemic men and women. Am J Clin Nutr 76, 365-372.

357. Maskarinec G, Steude JS, Franke AA, et al. (2009) Inflammatory markers in a 2-year soy intervention among premenopausal women. I Inflamm (Lond) 6, 9.

358. Matthan NR, Jalbert SM, Ausman LM, et al. (2007) Effect of soy protein from differently processed products on cardiovascular disease risk factors and vascular endothelial function in hypercholesterolemic subjects. Am J Clin Nutr 85, 960-966.

359. McVeigh BL, Dillingham BL, Lampe JW, et al. (2006) Effect of soy protein varying in isoflavone content on serum lipids in healthy young men. Am J Clin Nutr 83, 244-251.

360. Mukuddem-Petersen J, Stonehouse OW, Jerling JC, et al. (2007) Effects of a high walnut and high cashew nut diet on selected markers of the metabolic syndrome: a controlled feeding trial. Br J Nutr 97, 1144-1153.

361. Nasca MM, Zhou JR \& Welty FK (2008) Effect of soy nuts on adhesion molecules and markers of inflammation in hypertensive and normotensive postmenopausal women. Am J Cardiol 102, 84-86.

362. Ryan-Borchers TA, Park JS, Chew BP, et al. (2006) Soy isoflavones modulate immune function in healthy postmenopausal women. Am J Clin Nutr 83, 1118-1125.

363. Teede HJ, Dalais FS \& McGrath BP (2004) Dietary soy containing phytoestrogens does not have detectable estrogenic 
effects on hepatic protein synthesis in postmenopausal women. Am J Clin Nutr 79, 396-401.

364. Zemel MB, Sun X, Sobhani T, et al. (2010) Effects of dairy compared with soy on oxidative and inflammatory stress in overweight and obese subjects. Am J Clin Nutr 91, $16-22$.

365. Maskarinec G, Oum R, Chaptman AK, et al. (2009) Inflammatory markers in a randomised soya intervention among men. Br J Nutr 101, 1740-1744.

366. Napora JK, Short RG, Muller DC, et al. (2011) High-dose isoflavones do not improve metabolic and inflammatory parameters in androgen-deprived men with prostate cancer. J Androl 32, 40-48.

367. Jiang R, Jacobs DR Jr, Mayer-Davis E, et al. (2006) Nut and seed consumption and inflammatory markers in the multiethnic study of atherosclerosis. Am J Epidemiol $\mathbf{1 6 3}$, $222-231$.

368. Li TY, Brennan AM, Wedick NM, et al. (2009) Regular consumption of nuts is associated with a lower risk of cardiovascular disease in women with type 2 diabetes. J Nutr 139, 1333-1338.

369. Sari I, Baltaci Y, Bagci C, et al. (2010) Effect of pistachio diet on lipid parameters, endothelial function, inflammation, and oxidative status: a prospective study. Nutrition $\mathbf{2 6}$, 399-404.

370. Rajaram S, Connell KM \& Sabate J (2010) Effect of almondenriched high-monounsaturated fat diet on selected markers of inflammation: a randomised, controlled, crossover study. Br J Nutr 103, 907-912.

371. Banel DK \& Hu FB (2009) Effects of walnut consumption on blood lipids and other cardiovascular risk factors: a meta-analysis and systematic review. Am J Clin Nutr 90, $56-63$.

372. Ros E (2009) Nuts and novel biomarkers of cardiovascular disease. Am J Clin Nutr 89, 1649S-1656S.

373. Lopez-Garcia E, Schulze MB, Manson JE, et al. (2004) Consumption of $(n-3)$ fatty acids is related to plasma biomarkers of inflammation and endothelial activation in women. J Nutr 134, 1806-1811.

374. Madsen T, Skou HA, Hansen VE, et al. (2001) C-reactive protein, dietary $n-3$ fatty acids, and the extent of coronary artery disease. Am J Cardiol 88, 1139-1142.

375. King DE, Egan BM \& Geesey ME (2003) Relation of dietary fat and fiber to elevation of C-reactive protein. Am J Cardiol 92, 1335-1339.

376. Hickling S, Hung J, Knuiman M, et al. (2008) Are the associations between diet and C-reactive protein independent of obesity? Prev Med 47, 71-76.

377. Zampelas A, Panagiotakos DB, Pitsavos C, et al. (2005) Fish consumption among healthy adults is associated with decreased levels of inflammatory markers related to cardiovascular disease: the ATTICA study. J Am Coll Cardiol 46, 120-124.

378. Lindqvist H, Langkilde AM, Undeland I, et al. (2007) Herring (Clupea harengus) supplemented diet influences risk factors for CVD in overweight subjects. Eur J Clin Nutr 61, 1106-1113.

379. Maki T, Pham NM, Yoshida D, et al. (2010) The relationship of coffee and green tea consumption with high-sensitivity C-reactive protein in Japanese men and women. Clin Chem Lab Med 48, 849-854.

380. De Bacquer D, Clays E, Delanghe J, et al. (2006) Epidemiological evidence for an association between habitual tea consumption and markers of chronic inflammation. Atherosclerosis 189, 428-435.
381. Basu A, Du M, Sanchez K, et al. (2011) Green tea minimally affects biomarkers of inflammation in obese subjects with metabolic syndrome. Nutrition 27, 206-213.

382. de Maat MP, Pijl H, Kluft C, et al. (2000) Consumption of black and green tea had no effect on inflammation, haemostasis and endothelial markers in smoking healthy individuals. Eur J Clin Nutr 54, 757-763.

383. Fukino Y, Ikeda A, Maruyama K, et al. (2008) Randomized controlled trial for an effect of green tea-extract powder supplementation on glucose abnormalities. Eur J Clin Nutr 62, 953-960.

384. Hodgson JM, Puddey IB, Mori TA, et al. (2001) Effects of regular ingestion of black tea on haemostasis and cell adhesion molecules in humans. Eur J Clin Nutr 55, 881-886.

385. Mukamal KJ, MacDermott K, Vinson JA, et al. (2007) A 6-month randomized pilot study of black tea and cardiovascular risk factors. Am Heart J 154, 724-726.

386. Ryu OH, Lee J, Lee KW, et al. (2006) Effects of green tea consumption on inflammation, insulin resistance and pulse wave velocity in type 2 diabetes patients. Diabetes Res Clin Pract 71, 356-358.

387. Steptoe A, Gibson EL, Vuononvirta R, et al. (2007) The effects of chronic tea intake on platelet activation and inflammation: a double-blind placebo controlled trial. Atherosclerosis 193, 277-282.

388. Sung H, Min WK, Lee W, et al. (2005) The effects of green tea ingestion over four weeks on atherosclerotic markers. Ann Clin Biochem 42, 292-297.

389. Widlansky ME, Duffy SJ, Hamburg NM, et al. (2005) Effects of black tea consumption on plasma catechins and markers of oxidative stress and inflammation in patients with coronary artery disease. Free Radic Biol Med 38, 499-506.

390. Khan N, Afaq F, Saleem M, et al. (2006) Targeting multiple signaling pathways by green tea polyphenol (-)-epigallocatechin-3-gallate. Cancer Res 66, 2500-2505.

391. Kim SJ, Jeong HJ, Lee KM, et al. (2007) Epigallocatechin-3gallate suppresses NF-kappaB activation and phosphorylation of p38 MAPK and JNK in human astrocytoma U373MG cells. $J$ Nutr Biochem 18, 587-596.

392. Syed DN, Afaq F, Kweon MH, et al. (2007) Green tea polyphenol EGCG suppresses cigarette smoke condensateinduced NF-kappaB activation in normal human bronchial epithelial cells. Oncogene 26, 673-682.

393. Feng WY (2006) Metabolism of green tea catechins: an overview. Curr Drug Metab 7, 755-809.

394. Renouf M, Guy P, Marmet C, et al. (2010) Plasma appearance and correlation between coffee and green tea metabolites in human subjects. Br J Nutr 104, 1635-1640.

395. Hamer M, Williams ED, Vuononvirta R, et al. (2006) Association between coffee consumption and markers of inflammation and cardiovascular function during mental stress. J Hypertens 24, 2191-2197.

396. Lopez-Garcia E, van Dam RM, Qi L, et al. (2006) Coffee consumption and markers of inflammation and endothelial dysfunction in healthy and diabetic women. Am J Clin Nutr 84, 888-893.

397. Williams CJ, Fargnoli JL, Hwang JJ, et al. (2008) Coffee consumption is associated with higher plasma adiponectin concentrations in women with or without type 2 diabetes: a prospective cohort study. Diabetes Care 31, 504-507.

398. Zampelas A, Panagiotakos DB, Pitsavos C, et al. (2004) Associations between coffee consumption and inflammatory markers in healthy persons: the ATTICA study. $A m \mathrm{~J}$ Clin Nutr 80, 862-867. 
399. Rodrigues IM \& Klein LC (2006) Boiled or filtered coffee? Effects of coffee and caffeine on cholesterol, fibrinogen and C-reactive protein. Toxicol Rev 25, 55-69.

400. Bonita JS, Mandarano M, Shuta D, et al. (2007) Coffee and cardiovascular disease: in vitro, cellular, animal, and human studies. Pharmacol Res 55, 187-198.

401. Moisey LL, Kacker S, Bickerton AC, et al. (2008) Caffeinated coffee consumption impairs blood glucose homeostasis in response to high and low glycemic index meals in healthy men. Am J Clin Nutr 87, 1254-1261.

402. Lane JD, Feinglos MN \& Surwit RS (2008) Caffeine increases ambulatory glucose and postprandial responses in coffee drinkers with type 2 diabetes. Diabetes Care 31, 221-222.

403. Battram DS, Arthur R, Weekes A, et al. (2006) The glucose intolerance induced by caffeinated coffee ingestion is less pronounced than that due to alkaloid caffeine in men. J Nutr 136, 1276-1280.

404. Kempf K, Herder C, Erlund I, et al. (2010) Effects of coffee consumption on subclinical inflammation and other risk factors for type 2 diabetes: a clinical trial. Am J Clin Nutr 91, 950-957

405. Adamson GE, Lazarus SA, Mitchell AE, et al. (1999) HPLC method for the quantification of procyanidins in cocoa and chocolate samples and correlation to total antioxidant capacity. J Agric Food Chem 47, 4184-4188.

406. Hammerstone JF, Lazarus SA, Mitchell AE, et al. (1999) Identification of procyanidins in cocoa (Theobroma cacao) and chocolate using high-performance liquid chromatography/mass spectrometry. J Agric Food Chem 47, 490-496.

407. Natsume M, Osakabe N, Yamagishi M, et al. (2000) Analyses of polyphenols in cacao liquor, cocoa, and chocolate by normal-phase and reversed-phase HPLC. Biosci Biotechnol Biochem 64, 2581-2587.

408. Lazarus SA, Adamson GE, Hammerstone JF, et al. (1999) High-performance liquid chromatography/mass spectrometry analysis of proanthocyanidins in foods and beverages. J Agric Food Chem 47, 3693-3701.

409. Lazarus SA, Hammerstone JF \& Schmitz HH (1999) Chocolate contains additional flavonoids not found in tea. Lancet 354, 1825.

410. Vinson JA, Jang J, Dabbagh YA, et al. (1995) Plant polyphenols exhibit lipo-protein bound antioxidant activity using an in vitro oxidation model for heart disease. J Agric Food Chem 43, 2798-2799.

411. Hammerstone JF, Lazarus SA \& Schmitz HH (2000) Procyanidin content and variation in some commonly consumed foods. J Nutr 130, 2086S-2092S.

412. Mao T, Van de Water J, Keen CL, et al. (2000) Cocoa procyanidins and human cytokine transcription and secretion. J Nutr 130, 2093S-2099S.

413. Mao TK, Powell J, Van de Water J, et al. (2000) The effect of cocoa procyanidins on the transcription and secretion of interleukin 1 beta in peripheral blood mononuclear cells. Life Sci 66, 1377-1386.

414. di Giuseppe R, Di Castelnuovo A, Centritto F, et al. (2008) Regular consumption of dark chocolate is associated with low serum concentrations of C-reactive protein in a healthy Italian population. J Nutr 138, 1939-1945.

415. Mathur S, Devaraj S, Grundy SM, et al. (2002) Cocoa products decrease low density lipoprotein oxidative susceptibility but do not affect biomarkers of inflammation in humans. J Nutr 132, 3663-3667.

416. Hamed MS, Gambert S, Bliden KP, et al. (2008) Dark chocolate effect on platelet activity, C-reactive protein and lipid profile: a pilot study. South Med J 101, 1203-1208.
417. Monagas M, Khan N, Andres-Lacueva C, et al. (2009) Effect of cocoa powder on the modulation of inflammatory biomarkers in patients at high risk of cardiovascular disease. Am J Clin Nutr 90, 1144-1150.

418. Albert MA, Glynn RJ \& Ridker PM (2003) Alcohol consumption and plasma concentration of C-reactive protein. Circulation 107, 443-447.

419. Alho H, Sillanaukee P, Kalela A, et al. (2004) Alcohol misuse increases serum antibodies to oxidized LDL and C-reactive protein. Alcohol Alcohol 39, 312-315.

420. Averina M, Nilssen O, Arkhipovsky VL, et al. (2006) C-reactive protein and alcohol consumption: Is there a U-shaped association? Results from a population-based study in Russia. The Arkhangelsk study. Atherosclerosis 188, 309-315.

421. Bermudez EA, Rifai N, Buring J, et al. (2002) Interrelationships among circulating interleukin-6, C-reactive protein, and traditional cardiovascular risk factors in women. Arterioscler Thromb Vasc Biol 22, 1668-1673.

422. Englund OL, Brohall G, Behre CJ, et al. (2006) Alcohol consumption in relation to metabolic regulation, inflammation, and adiponectin in 64-year-old Caucasian women: a population-based study with a focus on impaired glucose regulation. Diabetes Care 29, 908-913.

423. Gonzalez-Quintela A, Alende R, Gude F, et al. (2008) Serum levels of immunoglobulins (IgG, IgA, IgM) in a general adult population and their relationship with alcohol consumption, smoking and common metabolic abnormalities. Clin Exp Immunol 151, 42-50.

424. Hoffmeister A, Imhof A, Rothenbacher D, et al. (2003) Moderate alcohol consumption and plasma concentration of sensitive markers of inflammation. Comment on an atheroprotective relationship. Dtsch Med Wochenschr 128, $2237-2241$.

425. Imhof A, Froehlich M, Brenner H, et al. (2001) Effect of alcohol consumption on systemic markers of inflammation. Lancet 357, 763-767.

426. Imhof A, Woodward M, Doering A, et al. (2004) Overall alcohol intake, beer, wine, and systemic markers of inflammation in western Europe: results from three MONICA samples (Augsburg, Glasgow, Lille). Eur Heart J 25, 2092-2100

427. Mukamal KJ, Cushman M, Mittleman MA, et al. (2004) Alcohol consumption and inflammatory markers in older adults: the Cardiovascular Health Study. Atherosclerosis 173, 79-87.

428. Pai JK, Hankinson SE, Thadhani R, et al. (2006) Moderate alcohol consumption and lower levels of inflammatory markers in US men and women. Atherosclerosis 186, 113-120.

429. Shai I, Rimm EB, Schulze MB, et al. (2004) Moderate alcohol intake and markers of inflammation and endothelial dysfunction among diabetic men. Diabetologia $\mathbf{4 7}$, $1760-1767$.

430. Stewart SH, Mainous AG III \& Gilbert G (2002) Relation between alcohol consumption and C-reactive protein levels in the adult US population. J Am Board Fam Pract 15, 437-442.

431. Volpato S, Pahor M, Ferrucci L, et al. (2004) Relationship of alcohol intake with inflammatory markers and plasminogen activator inhibitor-1 in well-functioning older adults: the Health, Aging, and Body Composition study. Circulation 109, 607-612

432. Wannamethee SG, Lowe GD, Shaper G, et al. (2003) The effects of different alcoholic drinks on lipids, insulin and 
haemostatic and inflammatory markers in older men. Thromb Haemost 90, 1080-1087.

433. Beulens JW, de Zoete EC, Kok FJ, et al. (2008) Effect of moderate alcohol consumption on adipokines and insulin sensitivity in lean and overweight men: a diet intervention study. Eur J Clin Nutr 62, 1098-1105.

434. Beulens JW, van den BR, Kok FJ, et al. (2008) Moderate alcohol consumption and lipoprotein-associated phospholipase A2 activity. Nutr Metab Cardiovasc Dis 18, 539-544.

435. Watzl B, Bub A, Briviba K, et al. (2002) Acute intake of moderate amounts of red wine or alcohol has no effect on the immune system of healthy men. Eur J Nutr 41, 264-270.

436. Watzl B, Bub A, Pretzer G, et al. (2004) Daily moderate amounts of red wine or alcohol have no effect on the immune system of healthy men. Eur J Clin Nutr 58, 40-45.

437. Beulens JW, van Beers RM, Stolk RP, et al. (2006) The effect of moderate alcohol consumption on fat distribution and adipocytokines. Obesity (Silver Spring) 14, 60-66.

438. Blanco-Colio LM, Valderrama M, Alvarez-Sala LA, et al. (2000) Red wine intake prevents nuclear factor-kappaB activation in peripheral blood mononuclear cells of healthy volunteers during postprandial lipemia. Circulation 102, 1020-1026.

439. Blann AD, Williams NR, Lip GY, et al. (2002) Acute ingestion of red wine by men activates platelets but does not influence endothelial markers: no effect of white wine. Blood Coagul Fibrinolysis 13, 647-651.

440. Dhindsa S, Tripathy D, Mohanty P, et al. (2004) Differential effects of glucose and alcohol on reactive oxygen species generation and intranuclear nuclear factor-kappaB in mononuclear cells. Metabolism 53, 330-334.

441. Gonzalez-Quintela A, Dominguez-Santalla MJ, Perez LF, et al. (2000) Influence of acute alcohol intake and alcohol withdrawal on circulating levels of IL-6, IL-8, IL-10 and IL-12. Cytokine 12, 1437-1440.

442. Greenfield JR, Samaras K, Hayward CS, et al. (2005) Beneficial postprandial effect of a small amount of alcohol on diabetes and cardiovascular risk factors: modification by insulin resistance. J Clin Endocrinol Metab 90, 661-672.

443. Pedersen N, Larsen S, Seidelin JB, et al. (2004) Alcohol modulates circulating levels of interleukin- 6 and monocyte chemoattractant protein-1 in chronic pancreatitis. Scand $J$ Gastroenterol 39, 277-282.

444. Retterstol L, Berge KE, Braaten O, et al. (2005) A daily glass of red wine: does it affect markers of inflammation? Alcohol 40, 102-105.

445. Sacanella E, Vazquez-Agell M, Mena MP, et al. (2007) Down-regulation of adhesion molecules and other inflammatory biomarkers after moderate wine consumption in healthy women: a randomized trial. Am J Clin Nutr 86, 1463-1469.

446. Sierksma A, van der Gaag MS, Kluft C, et al. (2002) Moderate alcohol consumption reduces plasma C-reactive protein and fibrinogen levels; a randomized, diet-controlled intervention study. Eur J Clin Nutr 56, 1130-1136.

447. Vazquez-Agell M, Sacanella E, Tobias E, et al. (2007) Inflammatory markers of atherosclerosis are decreased after moderate consumption of cava (sparkling wine) in men with low cardiovascular risk. J Nutr 137, 2279-2284.

448. Williams MJ, Sutherland WH, Whelan AP, et al. (2004) Acute effect of drinking red and white wines on circulating levels of inflammation-sensitive molecules in men with coronary artery disease. Metabolism 53, 318-323.
449. Vlassara H (2005) Advanced glycation in health and disease: role of the modern environment. Ann N Y Acad Sci 1043, 452-460.

450. Vlassara H \& Striker G (2007) Glycotoxins in the diet promote diabetes and diabetic complications. Curr Diab Rep 7, 235-241.

451. Ames JM (2007) Evidence against dietary advanced glycation endproducts being a risk to human health. Mol Nutr Food Res 51, 1085-1090.

452. Henle T (2007) Dietary advanced glycation end products a risk to human health? A call for an interdisciplinary debate. Mol Nutr Food Res 51, 1075-1078.

453. Sebekova K \& Somoza V (2007) Dietary advanced glycation endproducts (AGEs) and their health effects-PRO. Mol Nutr Food Res 51, 1079-1084.

454. Henle T (2003) AGEs in foods: do they play a role in uremia? Kidney Int Suppl 63, S145-S147.

455. Monnier VM (2003) Intervention against the Maillard reaction in vivo. Arch Biochem Biophys 419, 1-15.

456. Koschinsky T, He CJ, Mitsuhashi T, et al. (1997) Orally absorbed reactive glycation products (glycotoxins) an environmental risk factor in diabetic nephropathy. Proc Natl Acad Sci U S A 94, 6474-6479.

457. Sebekova K, Podracka L, Heidland A, et al. (2001) Enhanced plasma levels of advanced glycation end products (AGE) and pro-inflammatory cytokines in children/ adolescents with chronic renal insufficiency and after renal replacement therapy by dialysis and transplantation - are they inter-related? Clin Nephrol 56, S21-S26.

458. Thornalley PJ (2005) Glycation free adduct accumulation in renal disease: the new AGE. Pediatr Nephrol 20, $1515-1522$.

459. Uribarri J, Peppa M, Cai W, et al. (2003) Dietary glycotoxins correlate with circulating advanced glycation end product levels in renal failure patients. Am J Kidney Dis 42, 532-538.

460. Baynes JW \& Thorpe SR (1999) Role of oxidative stress in diabetic complications: a new perspective on an old paradigm. Diabetes $\mathbf{4 8}, 1-9$.

461. Goldberg T, Cai W, Peppa M, et al. (2004) Advanced glycoxidation end products in commonly consumed foods. J Am Diet Assoc 104, 1287-1291.

462. Cerami C, Founds H, Nicholl I, et al. (1997) Tobacco smoke is a source of toxic reactive glycation products. Proc Natl Acad Sci U S A 94, 13915-13920.

463. Erbersdobler HF \& Faist V (2001) Metabolic transit of Amadori products. Nabrung 45, 177-181.

464. Bergmann R, Helling R, Heichert C, et al. (2001) Radio fluorination and positron emission tomography (PET) as a new approach to study the in vivo distribution and elimination of the advanced glycation endproducts $\mathrm{N}$ epsilon-carboxymethyllysine (CML) and N epsilon-carboxyethyllysine (CEL). Nabrung 45, 182-188.

465. Finot PA \& Magnenat E (1981) Metabolic transit of early and advanced Maillard products. Prog Food Nutr Sci 5, 193-207.

466. Ahmed N (2005) Advanced glycation endproducts-role in pathology of diabetic complications. Diabetes Res Clin Pract 67, 3-21.

467. Hidalgo FJ \& Zamora R (2005) Interplay between the maillard reaction and lipid peroxidation in biochemical systems. Ann N Y Acad Sci 1043, 319-326.

468. Esterbauer H, Schaur RJ \& Zollner H (1991) Chemistry and biochemistry of 4-hydroxynonenal, malonaldehyde and related aldehydes. Free Radic Biol Med 11, 81-128. 
469. Uchida K (2000) Role of reactive aldehyde in cardiovascular diseases. Free Radic Biol Med 28, 1685-1696.

470. Fu MX, Requena JR, Jenkins AJ, et al. (1996) The advanced glycation end product, Nepsilon-(carboxymethyl)lysine, is a product of both lipid peroxidation and glycoxidation reactions. J Biol Chem 271, 9982-9986.

471. Brownlee M (1995) Advanced protein glycosylation in diabetes and aging. Annu Rev Med 46, 223-234.

472. Basta G, Lazzerini G, Del TS, et al. (2005) At least 2 distinct pathways generating reactive oxygen species mediate vascular cell adhesion molecule- 1 induction by advanced glycation end products. Arterioscler Thromb Vasc Biol 25, $1401-1407$.

473. Jay D, Hitomi H \& Griendling KK (2006) Oxidative stress and diabetic cardiovascular complications. Free Radic Biol Med 40, 183-192.

474. Bierhaus A, Humpert PM, Morcos M, et al. (2005) Understanding RAGE, the receptor for advanced glycation end products. J Mol Med 83, 876-886.

475. Bierhaus A, Rudofsky GJ \& Humpert PM (2005) The immune response to food and its impact on atherogenesis Diabetes Stoffwechsel Suppl. 1, 14-15.

476. Lander HM, Tauras JM, Ogiste JS, et al. (1997) Activation of the receptor for advanced glycation end products triggers a p21(ras)-dependent mitogen-activated protein kinase pathway regulated by oxidant stress. J Biol Chem $\mathbf{2 7 2}$, 17810-17814.

477. Ramasamy R, Yan SF \& Schmidt AM (2007) Arguing for the motion: yes, RAGE is a receptor for advanced glycation endproducts. Mol Nutr Food Res 51, 1111-1115.

478. Stern D, Yan SD, Yan SF, et al. (2002) Receptor for advanced glycation endproducts: a multiligand receptor magnifying cell stress in diverse pathologic settings. $A d v$ Drug Deliv Rev 54, 1615-1625.

479. Li YM, Mitsuhashi T, Wojciechowicz D, et al. (1996) Molecular identity and cellular distribution of advanced glycation endproduct receptors: relationship of p60 to OST-48 and p90 to 80K-H membrane proteins. Proc Natl Acad Sci U S A 93, 11047-11052.

480. Vlassara H, Li YM, Imani F, et al. (1995) Identification of galectin-3 as a high-affinity binding protein for advanced glycation end products (AGE): a new member of the AGE-receptor complex. Mol Med 1, 634-646.

481. Heizmann CW (2007) The mechanism by which dietary AGEs are a risk to human health is via their interaction with RAGE: arguing against the motion. Mol Nutr Food Res 51, 1116-1119.

482. Cai W, He JC, Zhu L, et al. (2006) Advanced glycation end product (AGE) receptor 1 suppresses cell oxidant stress and activation signaling via EGF receptor. Proc Natl Acad Sci U S A 103, 13801-13806.

483. Iacobini C, Amadio L, Oddi G, et al. (2003) Role of galectin-3 in diabetic nephropathy. J Am Soc Nephrol 14, S264-S270

484. Horiuchi S, Sakamoto Y \& Sakai M (2003) Scavenger receptors for oxidized and glycated proteins. Amino Acids 25, 283-292.

485. Greaves DR \& Gordon S (2005) Thematic review series: the immune system and atherogenesis. Recent insights into the biology of macrophage scavenger receptors. J Lipid Res $\mathbf{4 6}$ $11-20$.

486. Murphy JE, Tedbury PR, Homer-Vanniasinkam S, et al. (2005) Biochemistry and cell biology of mammalian scavenger receptors. Atherosclerosis 182, 1-15.
487. Moore KJ \& Freeman MW (2006) Scavenger receptors in atherosclerosis: beyond lipid uptake. Arterioscler Thromb Vasc Biol 26, 1702-1711.

488. Nagai R, Mera K, Nakajou K, et al. (2007) The ligand activity of AGE-proteins to scavenger receptors is dependent on their rate of modification by AGEs. Biochim Biophys Acta 1772, 1192-1198

489. Park L, Raman KG, Lee KJ, et al. (1998) Suppression of accelerated diabetic atherosclerosis by the soluble receptor for advanced glycation endproducts. Nat Med $\mathbf{4}$, 1025-1031.

490. Bucciarelli LG, Wendt T, Qu W, et al. (2002) RAGE blockade stabilizes established atherosclerosis in diabetic apolipoprotein E-null mice. Circulation 106, 2827-2835.

491. Flyvbjerg A, Denner L, Schrijvers BF, et al. (2004) Long-term renal effects of a neutralizing RAGE antibody in obese type 2 diabetic mice. Diabetes 53, 166-172.

492. Yamamoto Y, Kato I, Doi T, et al. (2001) Development and prevention of advanced diabetic nephropathy in RAGEoverexpressing mice. J Clin Invest 108, 261-268.

493. Wendt TM, Tanji N, Guo J, et al. (2003) RAGE drives the development of glomerulosclerosis and implicates podocyte activation in the pathogenesis of diabetic nephropathy. Am J Pathol 162, 1123-1137.

494. Bierhaus A, Haslbeck KM, Humpert PM, et al. (2004) Loss of pain perception in diabetes is dependent on a receptor of the immunoglobulin superfamily. J Clin Invest 114, $1741-1751$.

495. Haslbeck KM, Schleicher E, Bierhaus A, et al. (2005) The AGE/RAGE/NF-(kappa)B pathway may contribute to the pathogenesis of polyneuropathy in impaired glucose tolerance (IGT). Exp Clin Endocrinol Diabetes 113, 288-291.

496. Pugliese G, Pricci F, Iacobini C, et al. (2001) Accelerated diabetic glomerulopathy in galectin-3/AGE receptor 3 knockout mice. FASEB J 15, 2471-2479.

497. Iacobini C, Menini S, Oddi G, et al. (2004) Galectin-3/AGEreceptor 3 knockout mice show accelerated AGE-induced glomerular injury: evidence for a protective role of galectin-3 as an AGE receptor. FASEB J 18, 1773-1775.

498. Iacobini C, Oddi G, Menini S, et al. (2005) Development of age-dependent glomerular lesions in galectin-3/AGE-receptor-3 knockout mice. Am J Physiol Renal Physiol 289, F611-F621.

499. Arar C, Gaudin JC, Capron L, et al. (1998) Galectin-3 gene (LGALS3) expression in experimental atherosclerosis and cultured smooth muscle cells. FEBS Lett 430, 307-311.

500. Nachtigal M, Al-Assaad Z, Mayer EP, et al. (1998) Galectin-3 expression in human atherosclerotic lesions. Am J Pathol 152, 1199-1208.

501. Iacobini C, Menini S, Ricci C, et al. (2009) Accelerated lipidinduced atherogenesis in galectin-3-deficient mice: role of lipoxidation via receptor-mediated mechanisms. Arterioscler Thromb Vasc Biol 29, 831-836.

502. Lu C, He JC, Cai W, et al. (2004) Advanced glycation endproduct (AGE) receptor 1 is a negative regulator of the inflammatory response to AGE in mesangial cells. Proc Natl Acad Sci U S A 101, 11767-11772.

503. He CJ, Zheng F, Stitt A, et al. (2000) Differential expression of renal AGE-receptor genes in NOD mice: possible role in nonobese diabetic renal disease. Kidney Int 58, 1931-1940.

504. He CJ, Koschinsky T, Buenting C, et al. (2001) Presence of diabetic complications in type 1 diabetic patients correlates with low expression of mononuclear cell AGE-receptor-1 and elevated serum AGE. Mol Med 7, 159-168.

505. Finot PA, Bujard E, Mottu F, et al. (1977) Availability of the true Schiff's bases of lysine. Chemical evaluation of the 
Schiff's base between lysine and lactose in milk. Adv Exp Med Biol 86B, 343-365.

506. Vlassara H \& Palace MR (2003) Glycoxidation: the menace of diabetes and aging. Mt Sinai J Med 70, 232-241.

507. Hofmann SM, Dong HJ, Li Z, et al. (2002) Improved insulin sensitivity is associated with restricted intake of dietary glycoxidation products in the $\mathrm{db} / \mathrm{db}$ mouse. Diabetes 51, 2082-2089.

508. Sandu O, Song K, Cai W, et al. (2005) Insulin resistance and type 2 diabetes in high-fat-fed mice are linked to high glycotoxin intake. Diabetes 54, 2314-2319.

509. Peppa M, He C, Hattori M, et al. (2003) Fetal or neonatal low-glycotoxin environment prevents autoimmune diabetes in NOD mice. Diabetes 52, 1441-1448.

510. Zheng F, He C, Cai W, et al. (2002) Prevention of diabetic nephropathy in mice by a diet low in glycoxidation products. Diabetes Metab Res Rev 18, 224-237.

511. Lin RY, Choudhury RP, Cai W, et al. (2003) Dietary glycotoxins promote diabetic atherosclerosis in apolipoprotein E-deficient mice. Atherosclerosis 168, 213-220.

512. Lin RY, Reis ED, Dore AT, et al. (2002) Lowering of dietary advanced glycation endproducts (AGE) reduces neointimal formation after arterial injury in genetically hypercholesterolemic mice. Atherosclerosis 163, 303-311.

513. Peppa M, Brem H, Ehrlich P, et al. (2003) Adverse effects of dietary glycotoxins on wound healing in genetically diabetic mice. Diabetes 52, 2805-2813.

514. Negrean M, Stirban A, Stratmann B, et al. (2007) Effects of low- and high-advanced glycation endproduct meals on macro- and microvascular endothelial function and oxidative stress in patients with type 2 diabetes mellitus. Am J Clin Nutr 85, 1236-1243.

515. Peppa M, Uribarri J, Cai W, et al. (2004) Glycoxidation and inflammation in renal failure patients. Am J Kidney Dis $\mathbf{4 3}$, 690-695.

516. Schiekofer S, Franke S, Andrassy M, et al. (2006) Postprandial mononuclear NF-kappaB activation is independent of the AGE-content of a single meal. Exp Clin Endocrinol Diabetes 114, 160-167.

517. Sebekova K, Somoza V, Jarcuskova M, et al. (2009) Plasma advanced glycation end products are decreased in obese children compared with lean controls. Int J Pediatr Obes 4, 112-118.

518. Stirban A, Negrean M, Stratmann B, et al. (2006) Benfotiamine prevents macro- and microvascular endothelial dysfunction and oxidative stress following a meal rich in advanced glycation end products in individuals with type 2 diabetes. Diabetes Care 29, 2064-2071.

519. Uribarri J, Stirban A, Sander D, et al. (2007) Single oral challenge by advanced glycation end products acutely impairs endothelial function in diabetic and nondiabetic subjects. Diabetes Care 30, 2579-2582.

520. Uribarri J, Cai W, Peppa M, et al. (2007) Circulating glycotoxins and dietary advanced glycation endproducts: two links to inflammatory response, oxidative stress, and aging. J Gerontol A Biol Sci Med Sci 62, 427-433.

521. Vlassara H, Cai W, Crandall J, et al. (2002) Inflammatory mediators are induced by dietary glycotoxins, a major risk factor for diabetic angiopathy. Proc Natl Acad Sci US A 99, 15596-15601.

522. Venkatesan A, Hemalatha A, Bobby Z, et al. (2006) Nonenzymatic glycation of plasma proteins in smokers. Indian J Physiol Pharmacol 50, 403-408.

523. Uribarri J, Peppa M, Cai W, et al. (2003) Restriction of dietary glycotoxins reduces excessive advanced glycation end products in renal failure patients. J Am Soc Nephrol 14, 728-731.

524. Busch M, Franke S, Wolf G, et al. (2006) The advanced glycation end product N(epsilon)-carboxymethyllysine is not a predictor of cardiovascular events and renal outcomes in patients with type 2 diabetic kidney disease and hypertension. Am J Kidney Dis 48, 571-579.

525. Schwedler SB, Metzger T, Schinzel R, et al. (2002) Advanced glycation end products and mortality in hemodialysis patients. Kidney Int 62, 301-310.

526. Baynes JW (2007) Dietary ALEs are a risk to human healthNOT!. Mol Nutr Food Res 51, 1102-1106.

527. Tessier FJ \& Birlouez-Aragon I (2010) Health effects of dietary Maillard reaction products: the results of ICARE and other studies. Amino Acids (Epublication ahead of print version 15 October 2010).

528. Faist V \& Erbersdobler HF (2001) Metabolic transit and in vivo effects of melanoidins and precursor compounds deriving from the Maillard reaction. Ann Nutr Metab 45, $1-12$.

529. Somoza V, Wenzel E, Weiss C, et al. (2006) Dose-dependent utilisation of casein-linked lysinoalanine, N(epsilon)-fructoselysine and N(epsilon)-carboxymethyllysine in rats. $\mathrm{Mol}$ Nutr Food Res 50, 833-841.

530. Hultsch C, Hellwig M, Pawelke B, et al. (2006) Biodistribution and catabolism of 18F-labeled N-epsilon-fructoselysine as a model of Amadori products. Nucl Med Biol 33, 865-873.

531. Somoza V (2005) Five years of research on health risks and benefits of Maillard reaction products: an update. Mol Nutr Food Res 49, 663-672.

532. Foerster A \& Henle T (2003) Glycation in food and metabolic transit of dietary AGEs (advanced glycation endproducts): studies on the urinary excretion of pyrraline. Biochem Soc Trans 31, 1383-1385.

533. Miyata T, Ueda Y, Horie K, et al. (1998) Renal catabolism of advanced glycation end products: the fate of pentosidine. Kidney Int 53, 416-422.

534. Wiame E, Delpierre G, Collard F, et al. (2002) Identification of a pathway for the utilization of the Amadori product fructoselysine in Escherichia coli. J Biol Chem 277, 42523-42529.

535. Penfold SA, Coughlan MT, Patel SK, et al. (2010) Circulating high-molecular-weight RAGE ligands activate pathways implicated in the development of diabetic nephropathy. Kidney Int 78, 287-295.

536. Hammes HP, Du X, Edelstein D, et al. (2003) Benfotiamine blocks three major pathways of hyperglycemic damage and prevents experimental diabetic retinopathy. Nat Med 9, 294-299.

537. Mehta R, Shangari N \& O'Brien PJ (2008) Preventing cell death induced by carbonyl stress, oxidative stress or mitochondrial toxins with vitamin B anti-AGE agents. Mol Nutr Food Res 52, 379-385.

538. Thornalley PJ, Babaei-Jadidi R, Al AH, et al. (2007) High prevalence of low plasma thiamine concentration in diabetes linked to a marker of vascular disease. Diabetologia 50, 2164-2170.

539. Ostendorp T, Leclerc E, Galichet A, et al. (2007) Structural and functional insights into RAGE activation by multimeric S100B. EMBO J 26, 3868-3878.

540. Schleicher ED (2010) A race for RAGE ligands. Kidney Int 78, 234-236.

541. Xie J, Reverdatto S, Frolov A, et al. (2008) Structural basis for pattern recognition by the receptor for advanced glycation end products (RAGE). J Biol Chem 283, 27255-27269. 
542. Buetler TM, Latado H, Leclerc E, et al. (2011) Glycolaldehyde-modified beta-lactoglobulin AGEs are unable to stimulate inflammatory signaling pathways in RAGE-expressing human cell lines. Mol Nutr Food Res 55, 291-299.

543. Reznikov LL, Waksman J, Azam T, et al. (2004) Effect of advanced glycation end products on endotoxin-induced TNF-alpha, IL-1beta and IL-8 in human peripheral blood mononuclear cells. Clin Nephrol 61, 324-336.

544. Ahmed N, Mirshekar-Syahkal B, Kennish L, et al. (2005) Assay of advanced glycation endproducts in selected beverages and food by liquid chromatography with tandem mass spectrometric detection. Mol Nutr Food Res 49, 691-699.

545. Uribarri J, Woodruff S, Goodman S, et al. (2010) Advanced glycation end products in foods and a practical guide to their reduction in the diet. J Am Diet Assoc 110, 911-916.

546. Assar SH, Moloney C, Lima M, et al. (2009) Determination of Nepsilon-(carboxymethyl)lysine in food systems by ultra performance liquid chromatography-mass spectrometry. Amino Acids 36, 317-326.

547. Morales FJ, Somoza V \& Fogliano V (2010) Physiological relevance of dietary melanoidins. Amino Acids DOI: 10.1007/s00726-010-0774-1.

548. Chuyen NV, Arai H, Nakanishi T, et al. (2005) Are food advanced glycation end products toxic in biological systems? Ann N Y Acad Sci 1043, 467-473.

549. Rudofsky G Jr, Reismann P, Schiekofer S, et al. (2004) Reduction of postprandial hyperglycemia in patients with type 2 diabetes reduces NF-kappaB activation in PBMCs. Horm Metab Res 36, 630-638.

550. Monnier L, Mas E, Ginet C, et al. (2006) Activation of oxidative stress by acute glucose fluctuations compared with sustained chronic hyperglycemia in patients with type 2 diabetes. JAMA 295, 1681-1687.

551. Ceriello A (2005) Postprandial hyperglycemia and diabetes complications: is it time to treat? Diabetes 54, 1-7.

552. Giacco F \& Brownlee M (2010) Oxidative stress and diabetic complications. Circ Res 107, 1058-1070.

553. Schindhelm RK, Alssema M, Scheffer PG, et al. (2007) Fasting and postprandial glycoxidative and lipoxidative stress are increased in women with type 2 diabetes. Diabetes Care 30, 1789-1794.

554. Alipour A, Elte JW, van Zaanen HC, et al. (2007) Postprandial inflammation and endothelial dysfuction. Biochem Soc Trans 35, 466-469.

555. Sourris KC, Forbes JM \& Cooper ME (2008) Therapeutic interruption of advanced glycation in diabetic nephropathy: do all roads lead to Rome? Ann N Y Acad Sci 1126, 101-106.

556. Spiteller G (2008) Peroxyl radicals are essential reagents in the oxidation steps of the Maillard reaction leading to generation of advanced glycation end products. Ann N Y Acad Sci 1126, 128-133.

557. He C, Sabol J, Mitsuhashi T, et al. (1999) Dietary glycotoxins: inhibition of reactive products by aminoguanidine facilitates renal clearance and reduces tissue sequestration. Diabetes 48, 1308-1315.

558. Shangari N, Depeint F, Furrer R, et al. (2005) The effects of partial thiamin deficiency and oxidative stress (i.e. glyoxal and methylglyoxal) on the levels of alpha-oxoaldehyde plasma protein adducts in Fischer 344 rats. FEBS Lett 579, 5596-5602.

559. Shangari N, Depeint F, Furrer R, et al. (2007) A thermolyzed diet increases oxidative stress, plasma alpha-aldehydes and colonic inflammation in the rat. Chem Biol Interact 169 , $100-109$

560. Sebekova K, Krajcoviova-Kudlackova M, Schinzel R, et al. (2001) Plasma levels of advanced glycation end products in healthy, long-term vegetarians and subjects on a western mixed diet. Eur J Nutr 40, 275-281.

561. Schalkwijk CG (2006) Is reduction of AGEs in food a potential medical nutrition therapy: are AGEs the real culprits? IMARS Highlights 1, 8.

562. Tai AW \& Newkirk MM (2000) An autoantibody targeting glycated IgG is associated with elevated serum immune complexes in rheumatoid arthritis (RA). Clin Exp Immunol 120, 188-193.

563. Turk Z, Ljubic S, Turk N, et al. (2001) Detection of autoantibodies against advanced glycation endproducts and AGE-immune complexes in serum of patients with diabetes mellitus. Clin Chim Acta 303, 105-115.

564. Ames JM, Wynne A, Hofmann A, et al. (1999) The effect of a model melanoidin mixture on faecal bacterial populations in vitro. Br J Nutr 82, 489-495.

565. Dittrich R, Dragonas C, Kannenkeril D, et al. (2009) A diet rich in Maillard reaction products protects LDL against copper induced oxidation ex vivo, a human intervention trial. Food Res Int 42, 1315-1322.

566. Lindenmeier M, Faist V \& Hofmann T (2002) Structural and functional characterization of pronyl-lysine, a novel protein modification in bread crust melanoidins showing in vitro antioxidative and phase I/II enzyme modulating activity. J Agric Food Chem 50, 6997-7006.

567. Marko D, Habermeyer M, Kemeny M, et al. (2003) Maillard reaction products modulating the growth of human tumor cells in vitro. Chem Res Toxicol 16, 48-55.

568. Chuyen NV (2006) Toxicity of the AGEs generated from the Maillard reaction: on the relationship of food-AGEs and biological-AGEs. Mol Nutr Food Res 50, 1140-1149.

569. Calder PC (2003) n-3 Polyunsaturated fatty acids and inflammation: from molecular biology to the clinic. Lipids 38, 343-352.

570. Calder PC (2006) n-3 Polyunsaturated fatty acids, inflammation, and inflammatory diseases. Am J Clin Nutr 83, 1505S-1519S.

571. van den Berghe W, Vermeulen L, Delerive P, et al. (2003) A paradigm for gene regulation: inflammation, NF-kappaB and PPAR. Adv Exp Med Biol 544, 181-196.

572. Weigert C, Brodbeck K, Staiger H, et al. (2004) Palmitate, but not unsaturated fatty acids, induces the expression of interleukin-6 in human myotubes through proteasomedependent activation of nuclear factor-kappaB. J Biol Chem 279, 23942-23952.

573. Ajuwon KM \& Spurlock ME (2005) Palmitate activates the NF-kappaB transcription factor and induces IL-6 and TNFalpha expression in 3T3-L1 adipocytes. J Nutr $\mathbf{1 3 5}$, 1841-1846.

574. Lee JY, Plakidas A, Lee WH, et al. (2003) Differential modulation of Toll-like receptors by fatty acids: preferential inhibition by $n-3$ Polyunsaturated fatty acids. J Lipid Res 44, 479-486.

575. Weatherill AR, Lee JY, Zhao L, et al. (2005) Saturated and polyunsaturated fatty acids reciprocally modulate dendritic cell functions mediated through TLR4. J Immunol 174, 5390-5397.

576. Eschen O, Christensen JH, Toft E, et al. (2005) Soluble adhesion molecules and marine $n-3$ fatty acids in patients referred for coronary angiography. Atherosclerosis 180, $327-331$ 
577. Fernandez-Real JM, Broch M, Vendrell J, et al. (2003) Insulin resistance, inflammation, and serum fatty acid composition. Diabetes Care 26, 1362-1368.

578. Ferrucci L, Cherubini A, Bandinelli S, et al. (2006) Relationship of plasma polyunsaturated fatty acids to circulating inflammatory markers. J Clin Endocrinol Metab 91, 439-446.

579. Klein-Platat C, Drai J, Oujaa M, et al. (2005) Plasma fatty acid composition is associated with the metabolic syndrome and low-grade inflammation in overweight adolescents. Am J Clin Nutr 82, 1178-1184.

580. Lopez-Garcia E, Schulze MB, Meigs JB, et al. (2005) Consumption of trans fatty acids is related to plasma biomarkers of inflammation and endothelial dysfunction. J Nutr 135, $562-566$.

581. Niu K, Hozawa A, Kuriyama S, et al. (2006) Dietary longchain $n$ - 3 fatty acids of marine origin and serum C-reactive protein concentrations are associated in a population with a diet rich in marine products. Am J Clin Nutr 84, 223-229.

582. Petersson H, Basu S, Cederholm T, et al. (2008) Serum fatty acid composition and indices of stearoyl-CoA desaturase activity are associated with systemic inflammation: longitudinal analyses in middle-aged men. Br J Nutr 99, 1186-1189.

583. Pischon T, Hankinson SE, Hotamisligil GS, et al. (2003) Habitual dietary intake of $n-3$ and $n-6$ fatty acids in relation to inflammatory markers among US men and women. Circulation 108, 155-160.

584. Poudel-Tandukar K, Nanri A, Matsushita Y, et al. (2009) Dietary intakes of alpha-linolenic and linoleic acids are inversely associated with serum C-reactive protein levels among Japanese men. Nutr Res 29, 363-370.

585. Yli-Jama P, Seljeflot I, Meyer HE, et al. (2002) Serum nonesterified very long-chain PUFA are associated with markers of endothelial dysfunction. Atherosclerosis 164, 275-281.

586. Baer DJ, Judd JT, Clevidence BA, et al. (2004) Dietary fatty acids affect plasma markers of inflammation in healthy men fed controlled diets: a randomized crossover study. Am J Clin Nutr 79, 969-973.

587. Li G, Barnes D, Butz D, et al. (2005) 10t,12c-conjugated linoleic acid inhibits lipopolysaccharide-induced cyclooxygenase expression in vitro and in vivo. J Lipid Res 46, 2134-2142.

588. Li G, Dong B, Butz DE, et al. (2006) NF-kappaB independent inhibition of lipopolysaccharide-induced cyclooxygenase by a conjugated linoleic acid cognate, conjugated nonadecadienoic acid. Biochim Biophys Acta 1761, 969-972.

589. Moloney F, Toomey S, Noone E, et al. (2007) Antidiabetic effects of cis-9, trans-11-conjugated linoleic acid may be mediated via anti-inflammatory effects in white adipose tissue. Diabetes 56, 574-582.

590. Mullen A, Moloney F, Nugent AP, et al. (2007) Conjugated linoleic acid supplementation reduces peripheral blood mononuclear cell interleukin-2 production in healthy middle-aged males. J Nutr Biochem 18, 658-666.

591. Nugent AP, Roche HM, Noone EJ, et al. (2005) The effects of conjugated linoleic acid supplementation on immune function in healthy volunteers. Eur J Clin Nutr 59, 742-750.

592. Ramakers JD, Plat J, Sebedio JL, et al. (2005) Effects of the individual isomers cis-9,trans-11 vs. trans-10, cis-12 of conjugated linoleic acid (CLA) on inflammation parameters in moderately overweight subjects with LDL-phenotype B. Lipids 40, 909-918.

593. Riserus U, Basu S, Jovinge S, et al. (2002) Supplementation with conjugated linoleic acid causes isomer-dependent oxi- dative stress and elevated C-reactive protein: a potential link to fatty acid-induced insulin resistance. Circulation 106, 1925-1929.

594. Smedman A, Basu S, Jovinge S, et al. (2005) Conjugated linoleic acid increased C-reactive protein in human subjects. Br J Nutr 94, 791-795.

595. Song HJ, Grant I, Rotondo D, et al. (2005) Effect of CLA supplementation on immune function in young healthy volunteers. Eur J Clin Nutr 59, 508-517.

596. Tricon S, Burdge GC, Kew S, et al. (2004) Effects of cis9,trans-11 and trans-10,cis-12 conjugated linoleic acid on immune cell function in healthy humans. Am J Clin Nutr 80, 1626-1633.

597. Turpeinen AM, Basu S \& Mutanen M (1998) A high linoleic acid diet increases oxidative stress in vivo and affects nitric oxide metabolism in humans. Prostaglandins Leukot Essent Fatty Acids 59, 229-233.

598. Burdge GC \& Calder PC (2006) Dietary alpha-linolenic acid and health-related outcomes: a metabolic perspective. Nutr Res Rev 19, 26-52.

599. Bemelmans WJ, Lefrandt JD, Feskens EJ, et al. (2004) Increased alpha-linolenic acid intake lowers C-reactive protein, but has no effect on markers of atherosclerosis. Eur J Clin Nutr 58, 1083-1089.

600. Junker R, Pieke B, Schulte H, et al. (2001) Changes in hemostasis during treatment of hypertriglyceridemia with a diet rich in monounsaturated and $n-3$ polyunsaturated fatty acids in comparison with a low-fat diet. Thromb Res 101, 355-366.

601. Paschos GK, Rallidis LS, Liakos GK, et al. (2004) Background diet influences the anti-inflammatory effect of alpha-linolenic acid in dyslipidaemic subjects. BrJ Nutr 92, 649-655.

602. Paschos GK, Zampelas A, Panagiotakos DB, et al. (2007) Effects of flaxseed oil supplementation on plasma adiponectin levels in dyslipidemic men. Eur J Nutr 46, 315-320.

603. Rallidis LS, Paschos G, Liakos GK, et al. (2003) Dietary alpha-linolenic acid decreases C-reactive protein, serum amyloid $\mathrm{A}$ and interleukin-6 in dyslipidaemic patients. Atherosclerosis 167, 237-242.

604. Rallidis LS, Paschos G, Papaioannou ML, et al. (2004) The effect of diet enriched with alpha-linolenic acid on soluble cellular adhesion molecules in dyslipidaemic patients. Atherosclerosis 174, 127-132.

605. Thies F, Miles EA, Nebe-von-Caron G, et al. (2001) Influence of dietary supplementation with long-chain $n-3$ or $n-6$ polyunsaturated fatty acids on blood inflammatory cell populations and functions and on plasma soluble adhesion molecules in healthy adults. Lipids $\mathbf{3 6}$ 1183-1193.

606. Zhao G, Etherton TD, Martin KR, et al. (2004) Dietary alpha-linolenic acid reduces inflammatory and lipid cardiovascular risk factors in hypercholesterolemic men and women. J Nutr 134, 2991-2997.

607. Liou YA, King DJ, Zibrik D, et al. (2007) Decreasing linoleic acid with constant alpha-linolenic acid in dietary fats increases $(n-3)$ eicosapentaenoic acid in plasma phospholipids in healthy men. J Nutr 137, 945-952.

608. Calder PC (2009) Polyunsaturated fatty acids and inflammatory processes: new twists in an old tale. Biochimie $\mathbf{9 1}$ $791-795$.

609. Kelley DS, Taylor PC, Nelson GJ, et al. (1997) Effects of dietary arachidonic acid on human immune response. Lipids 32, 449-456.

610. Yaqoob P, Pala HS, Cortina-Borja M, et al. (2000) Encapsulated fish oil enriched in alpha-tocopherol alters plasma 
phospholipid and mononuclear cell fatty acid compositions but not mononuclear cell functions. Eur J Clin Invest 30, $260-274$

611. Healy DA, Wallace FA, Miles EA, et al. (2000) Effect of lowto-moderate amounts of dietary fish oil on neutrophil lipid composition and function. Lipids 35, 763-768.

612. Rees D, Miles EA, Banerjee T, et al. (2006) Dose-related effects of eicosapentaenoic acid on innate immune function in healthy humans: a comparison of young and older men. Am J Clin Nutr 83, 331-342.

613. Calder PC (2007) Immunomodulation by omega-3 fatty acids. Prostaglandins Leukot Essent Fatty Acids 77, 327-335.

614. Calder PC (2008) The relationship between the fatty acid composition of immune cells and their function. Prostaglandins Leukot Essent Fatty Acids 79, 101-108.

615. Calder PC (2008) PUFA, inflammatory processes and rheumatoid arthritis. Proc Nutr Soc 67, 409-418.

616. Calder PC (2008) Polyunsaturated fatty acids, inflammatory processes and inflammatory bowel diseases. Mol Nutr Food Res 52, 885-897.

617. Kremmyda LS, Vlachava M, Noakes PS, et al. (2009) Atopy risk in infants and children in relation to early exposure to fish, oily fish, or long-chain omega-3 fatty acids: a systematic review. Clin Rev Allergy Immunol 41, 36-66.

618. Abe Y, El-Masri B, Kimball KT, et al. (1998) Soluble cell adhesion molecules in hypertriglyceridemia and potential significance on monocyte adhesion. Arterioscler Thromb Vasc Biol 18, 723-731.

619. Berstad P, Seljeflot I, Veierod MB, et al. (2003) Supplementation with fish oil affects the association between very long-chain $n$-3 Polyunsaturated fatty acids in serum non-esterified fatty acids and soluble vascular cell adhesion molecule-1. Clin Sci (Lond) 105, 13-20.

620. Blok WL, Deslypere JP, Demacker PN, et al. (1997) Pro- and anti-inflammatory cytokines in healthy volunteers fed various doses of fish oil for 1 year. Eur J Clin Invest 27, $1003-1008$.

621. Browning LM, Krebs JD, Moore CS, et al. (2007) The impact of long chain $n-3$ Polyunsaturated fatty acid supplementation on inflammation, insulin sensitivity and CVD risk in a group of overweight women with an inflammatory phenotype. Diabetes Obes Metab 9, 70-80.

622. Cazzola R, Russo-Volpe S, Miles EA, et al. (2007) Age- and dose-dependent effects of an eicosapentaenoic acid-rich oil on cardiovascular risk factors in healthy male subjects. Atherosclerosis 193, 159-167.

623. Chan DC, Watts GF, Mori TA, et al. (2002) Factorial study of the effects of atorvastatin and fish oil on dyslipidaemia in visceral obesity. Eur J Clin Invest 32, 429-436.

624. Ciubotaru I, Lee YS \& Wander RC (2003) Dietary fish oil decreases C-reactive protein, interleukin-6, and triacylglycerol to HDL-cholesterol ratio in postmenopausal women on HRT. J Nutr Biochem 14, 513-521.

625. Eschen O, Christensen JH, De CR, et al. (2004) Soluble adhesion molecules in healthy subjects: a dose-response study using $n-3$ fatty acids. Nutr Metab Cardiovasc Dis 14, 180-185.

626. Fujioka S, Hamazaki K, Itomura M, et al. (2006) The effects of eicosapentaenoic acid-fortified food on inflammatory markers in healthy subjects - a randomized, placebocontrolled, double-blind study. J Nutr Sci Vitaminol (Tokyo) 52, 261-265.

627. Grundt H, Nilsen DW, Mansoor MA, et al. (2003) Reduction in homocysteine by $n-3$ Polyunsaturated fatty acids after 1 year in a randomised double-blind study following an acute myocardial infarction: no effect on endothelial adhesion properties. Pathophysiol Haemost Thromb 33, $88-95$.

628. Hjerkinn EM, Seljeflot I, Ellingsen I, et al. (2005) Influence of long-term intervention with dietary counseling, longchain $n$-3 fatty acid supplements, or both on circulating markers of endothelial activation in men with long-standing hyperlipidemia. Am J Clin Nutr 81, 583-589.

629. Johansen O, Seljeflot I, Hostmark AT, et al. (1999) The effect of supplementation with omega- 3 fatty acids on soluble markers of endothelial function in patients with coronary heart disease. Arterioscler Thromb Vasc Biol 19, 1681-1686.

630. Kabir M, Skurnik G, Naour N, et al. (2007) Treatment for 2 mo with $n 3$ polyunsaturated fatty acids reduces adiposity and some atherogenic factors but does not improve insulin sensitivity in women with type 2 diabetes: a randomized controlled study. Am J Clin Nutr 86, 1670-1679.

631. Kelley DS, Siegel D, Fedor DM, et al. (2009) DHA supplementation decreases serum C-reactive protein and other markers of inflammation in hypertriglyceridemic men. J Nutr 139, 495-501.

632. Krebs JD, Browning LM, McLean NK, et al. (2006) Additive benefits of long-chain $n$-3 polyunsaturated fatty acids and weight-loss in the management of cardiovascular disease risk in overweight hyperinsulinaemic women. Int $J$ Obes (Lond) 30, 1535-1544.

633. Madsen T, Christensen JH, Blom M, et al. (2003) The effect of dietary $n$ - 3 fatty acids on serum concentrations of C-reactive protein: a dose-response study. Br J Nutr 89, 517-522.

634. Madsen T, Schmidt EB \& Christensen JH (2007) The effect of $n-3$ fatty acids on C-reactive protein levels in patients with chronic renal failure. J Ren Nutr 17, 258-263.

635. Madsen T, Christensen JH \& Schmidt EB (2007) C-reactive protein and $n-3$ fatty acids in patients with a previous myocardial infarction: a placebo-controlled randomized study. Eur J Nutr 46, 428-430.

636. Mori TA, Woodman RJ, Burke V, et al. (2003) Effect of eicosapentaenoic acid and docosahexaenoic acid on oxidative stress and inflammatory markers in treated-hypertensive type 2 diabetic subjects. Free Radic Biol Med 35, 772-781.

637. Murphy KJ, Meyer BJ, Mori TA, et al. (2007) Impact of foods enriched with $n$-3 long-chain polyunsaturated fatty acids on erythrocyte $n$-3 levels and cardiovascular risk factors. $B r J$ Nutr 97, 749-757.

638. Pot GK, Brouwer IA, Enneman A, et al. (2009) No effect of fish oil supplementation on serum inflammatory markers and their interrelationships: a randomized controlled trial in healthy, middle-aged individuals. Eur J Clin Nutr 63, 1353-1359.

639. Rasic-Milutinovic Z, Perunicic G, Pljesa S, et al. (2007) Effects of $N-3$ PUFAs supplementation on insulin resistance and inflammatory biomarkers in hemodialysis patients. Ren Fail 29, 321-329.

640. Sampson MJ, Davies IR, Brown JC, et al. (2001) n-3 polyunsaturated fatty acid supplementation, monocyte adhesion molecule expression and pro-inflammatory mediators in type 2 diabetes mellitus. Diabet Med 18, 51-58.

641. Sanders TA, Lewis F, Slaughter S, et al. (2006) Effect of varying the ratio of $n-6$ to $n-3$ fatty acids by increasing the dietary intake of alpha-linolenic acid, eicosapentaenoic and docosahexaenoic acid, or both on fibrinogen and clotting factors VII and XII in persons aged $45-70$ y: the OPTILIP study. Am J Clin Nutr 84, 513-522. 
642. Seljeflot I, Arnesen H, Brude IR, et al. (1998) Effects of omega-3 fatty acids and/or antioxidants on endothelial cell markers. Eur J Clin Invest 28, 629-635.

643. Troseid M, Arnesen H, Hjerkinn EM, et al. (2009) Serum levels of interleukin-18 are reduced by diet and $n-3$ fatty acid intervention in elderly high-risk men. Metabolism 58, 1543-1549.

644. Woodman RJ, Mori TA, Burke V, et al. (2003) Effects of purified eicosapentaenoic acid and docosahexaenoic acid on platelet, fibrinolytic and vascular function in hypertensive type 2 diabetic patients. Atherosclerosis 166, 85-93.

645. Yamada H, Yoshida M, Nakano Y, et al. (2008) In vivo and in vitro inhibition of monocyte adhesion to endothelial cells and endothelial adhesion molecules by eicosapentaenoic acid. Arterioscler Thromb Vasc Biol 28, 2173-2179.

646. Yusof HM, Miles EA \& Calder PC (2008) Influence of very long-chain $\mathrm{n}-3$ fatty acids on plasma markers of inflammation in middle-aged men. Prostaglandins Leukot Essent Fatty Acids 78, 219-228.

647. Todoric J, Loffler M, Huber J, et al. (2006) Adipose tissue inflammation induced by high-fat diet in obese diabetic mice is prevented by $n-3$ polyunsaturated fatty acids. Diabetologia 49, 2109-2119.

648. Rossi AS, Lombardo YB, Lacorte JM, et al. (2005) Dietary fish oil positively regulates plasma leptin and adiponectin levels in sucrose-fed, insulin-resistant rats. Am J Physiol Regul Integr Comp Physiol 289, R486-R494.

649. Grimble RF, Howell WM, O'Reilly G, et al. (2002) The ability of fish oil to suppress tumor necrosis factor alpha production by peripheral blood mononuclear cells in healthy men is associated with polymorphisms in genes that influence tumor necrosis factor alpha production. Am J Clin Nutr 76, 454-459.

650. Shen J, Arnett DK, Peacock JM, et al. (2007) Interleukin1beta genetic polymorphisms interact with polyunsaturated fatty acids to modulate risk of the metabolic syndrome. J Nutr 137, 1846-1851.

651. Ceriello A, Quagliaro L, Piconi L, et al. (2004) Effect of postprandial hypertriglyceridemia and hyperglycemia on circulating adhesion molecules and oxidative stress generation and the possible role of simvastatin treatment. Diabetes 53, 701-710.

652. Ceriello A, Taboga C, Tonutti L, et al. (2002) Evidence for an independent and cumulative effect of postprandial hypertriglyceridemia and hyperglycemia on endothelial dysfunction and oxidative stress generation: effects of short- and long-term simvastatin treatment. Circulation 106, 1211-1218.

653. Kempf K, Rose B, Herder C, et al. (2007) The metabolic syndrome sensitizes leukocytes for glucose-induced immune gene expression. J Mol Med 85, 389-396.

654. Giugliano D, Ceriello A \& Esposito K (2006) The effects of diet on inflammation: emphasis on the metabolic syndrome. J Am Coll Cardiol 48, 677-685.

655. Davidson SM \& Duchen MR (2007) Endothelial mitochondria: contributing to vascular function and disease. Circ Res 100, 1128-1141.

656. Mohan IK \& Das UN (1998) Effect of L-arginine-nitric oxide system on chemical-induced diabetes mellitus. Free Radic Biol Med 25, 757-765.

657. Williams SB, Goldfine AB, Timimi FK, et al. (1998) Acute hyperglycemia attenuates endothelium-dependent vasodilation in humans in vivo. Circulation 97, 1695-1701.

658. Scognamiglio R, Negut C, De Kreutzenberg SV, et al. (2005) Postprandial myocardial perfusion in healthy subjects and in type 2 diabetic patients. Circulation 112, 179-184.
659. Jacob S, Ruus P, Hermann R, et al. (1999) Oral administration of RAC-alpha-lipoic acid modulates insulin sensitivity in patients with type- 2 diabetes mellitus: a placebo-controlled pilot trial. Free Radic Biol Med 27, 309-314.

660. Caballero B (1993) Vitamin E improves the action of insulin. Nutr Rev 51, 339-340.

661. May JM (2000) How does ascorbic acid prevent endothelial dysfunction? Free Radic Biol Med 28, 1421-1429.

662. Evans JL, Goldfine ID, Maddux BA, et al. (2002) Oxidative stress and stress-activated signaling pathways: a unifying hypothesis of type 2 diabetes. Endocr Rev 23, 599-622.

663. Giugliano D, Marfella R, Coppola L, et al. (1997) Vascular effects of acute hyperglycemia in humans are reversed by L-arginine. Evidence for reduced availability of nitric oxide during hyperglycemia. Circulation 95, 1783-1790.

664. Ceriello A, Bortolotti N, Motz E, et al. (1999) Meal-induced oxidative stress and low-density lipoprotein oxidation in diabetes: the possible role of hyperglycemia. Metabolism 48, 1503-1508.

665. Ursini F, Zamburlini A, Cazzolato G, et al. (1998) Postprandial plasma lipid hydroperoxides: a possible link between diet and atherosclerosis. Free Radic Biol Med 25, 250-252.

666. Marfella R, Quagliaro L, Nappo F, et al. (2001) Acute hyperglycemia induces an oxidative stress in healthy subjects. J Clin Invest 108, 635-636.

667. Price KD, Price CS \& Reynolds RD (2001) Hyperglycemiainduced ascorbic acid deficiency promotes endothelial dysfunction and the development of atherosclerosis. Atherosclerosis 158, 1-12.

668. Chen MS, Hutchinson ML, Pecoraro RE, et al. (1983) Hyperglycemia-induced intracellular depletion of ascorbic acid in human mononuclear leukocytes. Diabetes 32, 1078-1081.

669. Beisswenger PJ, Howell SK, O’Dell RM, et al. (2001) alphaDicarbonyls increase in the postprandial period and reflect the degree of hyperglycemia. Diabetes Care 24, 726-732.

670. Cai W, He JC, Zhu L, et al. (2004) High levels of dietary advanced glycation end products transform low-density lipoprotein into a potent redox-sensitive mitogen-activated protein kinase stimulant in diabetic patients. Circulation 110, 285-291.

671. Kempf K, Rose B, Herder C, et al. (2006) Inflammation in metabolic syndrome and type 2 diabetes: impact of dietary glucose. Ann N Y Acad Sci 1084, 30-48.

672. Levitan EB, Cook NR, Stampfer MJ, et al. (2008) Dietary glycemic index, dietary glycemic load, blood lipids, and C-reactive protein. Metabolism 57, 437-443.

673. Du H, van der AD, van Bakel MM, et al. (2008) Glycemic index and glycemic load in relation to food and nutrient intake and metabolic risk factors in a Dutch population. Am J Clin Nutr 87, 655-661.

674. Pittas AG, Roberts SB, Das SK, et al. (2006) The effects of the dietary glycemic load on type 2 diabetes risk factors during weight loss. Obesity (Silver Spring) 14, 2200-2209.

675. Shikany JM, Phadke RP, Redden DT, et al. (2009) Effects of low- and high-glycemic index/glycemic load diets on coronary heart disease risk factors in overweight/obese men. Metabolism 58, 1793-1801.

676. Vrolix R \& Mensink RP (2010) Effects of glycemic load on metabolic risk markers in subjects at increased risk of developing metabolic syndrome. Am J Clin Nutr 92, 366-374.

677. Ajani UA, Ford ES \& Mokdad AH (2004) Dietary fiber and C-reactive protein: findings from national health and nutrition examination survey data. J Nutr 134, 1181-1185. 
678. King DE, Mainous AG III, Egan BM, et al. (2005) Fiber and C-reactive protein in diabetes, hypertension, and obesity. Diabetes Care 28, 1487-1489.

679. Bo S, Durazzo M, Guidi S, et al. (2006) Dietary magnesium and fiber intakes and inflammatory and metabolic indicators in middle-aged subjects from a populationbased cohort. Am J Clin Nutr 84, 1062-1069.

680. Ma Y, Griffith JA, Chasan-Taber L, et al. (2006) Association between dietary fiber and serum C-reactive protein. $\mathrm{Am} \mathrm{J}$ Clin Nutr 83, 760-766.

681. Ma Y, Hebert JR, Li W, et al. (2008) Association between dietary fiber and markers of systemic inflammation in the Women's Health Initiative Observational Study. Nutrition 24, 941-949.

682. King DE, Egan BM, Woolson RF, et al. (2007) Effect of a high-fiber diet vs a fiber-supplemented diet on C-reactive protein level. Arch Intern Med 167, 502-506.

683. Zemel MB \& Sun X (2008) Dietary calcium and dairy products modulate oxidative and inflammatory stress in mice and humans. J Nutr 138, 1047-1052.

684. Jauhiainen T, Vapaatalo H, Poussa T, et al. (2005) Lactobacillus helveticus fermented milk lowers blood pressure in hypertensive subjects in 24-h ambulatory blood pressure measurement. Am J Hypertens 18, 1600-1605.

685. Pins JJ \& Keenan JM (2006) Effects of whey peptides on cardiovascular disease risk factors. J Clin Hypertens (Greenwich) 8, 775-782.

686. Lee YM, Skurk T, Hennig M, et al. (2007) Effect of a milk drink supplemented with whey peptides on blood pressure in patients with mild hypertension. Eur J Nutr 46, 21-27.

687. Crouch SP, Slater KJ \& Fletcher J (1992) Regulation of cytokine release from mononuclear cells by the iron-binding protein lactoferrin. Blood 80, 235-240.

688. Conneely OM (2001) Antiinflammatory activities of lactoferrin. J Am Coll Nutr 20, 389S-395S.

689. Legrand D, Elass E, Carpentier M, et al. (2005) Lactoferrin: a modulator of immune and inflammatory responses. Cell Mol Life Sci 62, 2549-2559.

690. Ahluwalia N (1998) Diagnostic utility of serum transferrin receptors measurement in assessing iron status. Nutr Rev 56, 133-141

691. Weiss G \& Goodnough LT (2005) Anemia of chronic disease. N Engl J Med 352, 1011-1023.

692. Baynes RD (1996) Assessment of iron status. Clin Biochem 29, 209-215.

693. Cook JD, Flowers CH \& Skikne BS (2003) The quantitative assessment of body iron. Blood 101, 3359-3364

694. Northrop-Clewes CA (2008) Interpreting indicators of iron status during an acute phase response-lessons from malaria and human immunodeficiency virus. Ann Clin Biochem 45, 18-32.

695. Weiss G (2002) Iron and immunity: a double-edged sword Eur J Clin Invest 32, Suppl. 1, 70-78.

696. Ahluwalia N, Sun J, Krause D, et al. (2004) Immune function is impaired in iron-deficient, homebound, older women. Am J Clin Nutr 79, 516-521.

697. de Silva A, Atukorala S, Weerasinghe I, et al. (2003) Iron supplementation improves iron status and reduces morbidity in children with or without upper respiratory tract infections: a randomized controlled study in Colombo, Sri Lanka. Am J Clin Nutr 77, 234-241.

698. Wood RJ (2004) The iron-heart disease connection: is it dead or just hiding? Ageing Res Rev 3, 355-367.

699. Wagener FA, Volk HD, Willis D, et al. (2003) Different faces of the heme-heme oxygenase system in inflammation. Pharmacol Rev 55, 551-571.
700. McCord JM (1998) Iron, free radicals, and oxidative injury. Semin Hematol 35, 5-12.

701. Lee DH \& Jacobs DR Jr (2004) Serum markers of stored body iron are not appropriate markers of health effects of iron: a focus on serum ferritin. Med Hypotheses 62, 442-445.

702. Rajpathak S, Ma J, Manson J, et al. (2006) Iron intake and the risk of type 2 diabetes in women: a prospective cohort study. Diabetes Care 29, 1370-1376.

703. Ascherio A, Willett WC, Rimm EB, et al. (1994) Dietary iron intake and risk of coronary disease among men. Circulation 89, 969-974.

704. Jiang R, Ma J, Ascherio A, et al. (2004) Dietary iron intake and blood donations in relation to risk of type 2 diabetes in men: a prospective cohort study. Am J Clin Nutr 79, $70-75$.

705. Wrede CE, Buettner R, Bollheimer LC, et al. (2006) Association between serum ferritin and the insulin resistance syndrome in a representative population. Eur J Endocrinol 154, 333-340.

706. Fernandez-Real JM, Moreno JM, Lopez-Bermejo A, et al. (2007) Circulating soluble transferrin receptor according to glucose tolerance status and insulin sensitivity. Diabetes Care 30, 604-608.

707. Jehn M, Clark JM \& Guallar E (2004) Serum ferritin and risk of the metabolic syndrome in U.S. adults. Diabetes Care 27, 2422-2428.

708. Alissa EM, Ahmed WH, Al-Ama N, et al. (2007) Relationship between indices of iron status and coronary risk factors including diabetes and the metabolic syndrome in Saudi subjects without overt coronary disease. I Trace Elem Med Biol 21, 242-254.

709. Sullivan JL (1989) The iron paradigm of ischemic heart disease. Am Heart J 117, 1177-1188.

710. Salonen JT, Nyyssonen K, Korpela H, et al. (1992) High stored iron levels are associated with excess risk of myocardial infarction in eastern Finnish men. Circulation 86, 803-811.

711. Kiechl S, Willeit J, Egger G, et al. (1997) Body iron stores and the risk of carotid atherosclerosis: prospective results from the Bruneck study. Circulation 96, 3300-3307.

712. Ramakrishnan U, Kuklina E \& Stein AD (2002) Iron stores and cardiovascular disease risk factors in women of reproductive age in the United States. Am J Clin Nutr 76, 1256-1260.

713. Danesh J \& Appleby P (1999) Coronary heart disease and iron status: meta-analyses of prospective studies. Circulation 99, 852-854.

714. Ahluwalia N, Genoux A, Ferrieres J, et al. (2010) Iron status is associated with carotid atherosclerotic plaques in middleaged adults. J Nutr 140, 812-816.

715. Facchini FS \& Saylor KL (2002) Effect of iron depletion on cardiovascular risk factors: studies in carbohydrateintolerant patients. Ann N Y Acad Sci 967, 342-351.

716. Selzer CC \& Mayer J (1963) Serum iron and iron-binding capacity in adolescents. II. Comparison of obese and nonobese subects. Am J Clin Nutr 13, 354-361.

717. Nead KG, Halterman JS, Kaczorowski JM, et al. (2004) Overweight children and adolescents: a risk group for iron deficiency. Pediatrics 114, 104-108.

718. Micozzi MS, Albanes D \& Stevens RG (1989) Relation of body size and composition to clinical biochemical and hematologic indices in US men and women. Am J Clin Nutr 50, 1276-1281.

719. Gillum RF (2001) Association of serum ferritin and indices of body fat distribution and obesity in Mexican American 
men - the Third National Health and Nutrition Examination Survey. Int J Obes Relat Metab Disord 25, 639-645.

720. Lecube A, Carrera A, Losada E, et al. (2006) Iron deficiency in obese postmenopausal women. Obesity (Silver Spring) 14, 1724-1730.

721. Yanoff LB, Menzie CM, Denkinger B, et al. (2007) Inflammation and iron deficiency in the hypoferremia of obesity. Int J Obes (Lond) 31, 1412-1419.

722. Anty R, Dahman M, Iannelli A, et al. (2008) Bariatric surgery can correct iron depletion in morbidly obese women: a link with chronic inflammation. Obes Surg 18, 709-714.

723. Hodgson JM, Ward NC, Burke V, et al. (2007) Increased lean red meat intake does not elevate markers of oxidative stress and inflammation in humans. J Nutr 137, 363-367.

724. Rosales FJ, Kang Y, Pfeiffer B, et al. (2004) Twice the recommended daily allowance of iron is associated with an increase in plasma [alpha]-1 antichymotrypsin concentrations in Guatemalan school-aged children. Nutr Res 24, 875-887.

725. Schumann K, Kroll S, Weiss G, et al. (2005) Monitoring of hematological, inflammatory and oxidative reactions to acute oral iron exposure in human volunteers: preliminary screening for selection of potentially-responsive biomarkers. Toxicology 212, 10-23.

726. Krafft A, Perewusnyk G, Hanseler E, et al. (2005) Effect of postpartum iron supplementation on red cell and iron parameters in non-anaemic iron-deficient women: a randomised placebo-controlled study. BJOG 112, 445-450.

727. Froicu M, Zhu Y \& Cantorna MT (2006) Vitamin D receptor is required to control gastrointestinal immunity in IL-10 knockout mice. Immunology 117, 310-318.

728. Baeke F, van EE, Gysemans C, et al. (2008) Vitamin D signaling in immune-mediated disorders: evolving insights and therapeutic opportunities. Mol Aspects Med 29, $376-387$.

729. Sun X \& Zemel MB (2007) Calcium and 1,25-dihydroxyvitamin D3 regulation of adipokine expression. Obesity (Silver Spring) 15, 340-348.

730. Borradale D \& Kimlin M (2009) Vitamin D in health and disease: an insight into traditional functions and new roles for the 'sunshine vitamin'. Nutr Res Rev 22, 118-136.

731. Talmor Y, Golan E, Benchetrit S, et al. (2008) Calcitriol blunts the deleterious impact of advanced glycation end products on endothelial cells. Am J Physiol Renal Physiol 294, F1059-F1064.

732. Martinesi M, Treves C, d'Albasio G, et al. (2008) Vitamin D derivatives induce apoptosis and downregulate ICAM-1 levels in peripheral blood mononuclear cells of inflammatory bowel disease patients. Inflamm Bowel Dis 14, 597-604.

733. Giulietti A, van EE, Overbergh L, et al. (2007) Monocytes from type 2 diabetic patients have a pro-inflammatory profile. 1,25-Dihydroxyvitamin D(3) works as anti-inflammatory. Diabetes Res Clin Pract 77, 47-57.

734. Cohen-Lahav M, Douvdevani A, Chaimovitz C, et al. (2007) The anti-inflammatory activity of 1,25-dihydroxyvitamin D3 in macrophages. J Steroid Biochem Mol Biol 103, 558-562.

735. Sadeghi K, Wessner B, Laggner U, et al. (2006) Vitamin D3 down-regulates monocyte TLR expression and triggers hyporesponsiveness to pathogen-associated molecular patterns. Eur J Immunol 36, 361-370.

736. Hewison M (2008) Vitamin D and innate immunity. Curr Opin Investig Drugs 9, 485-490.

737. Do JE, Kwon SY, Park S, et al. (2008) Effects of vitamin D on expression of Toll-like receptors of monocytes from patients with Behcet's disease. Rheumatology (Oxford) 47, 840-848.

738. Aparna R, Subhashini J, Roy KR, et al. (2008) Selective inhibition of cyclooxygenase-2 (COX-2) by 1alpha, 25-dihydroxy-16-ene-23-yne-vitamin D3, a less calcemic vitamin D analog. J Cell Biochem 104, 1832-1842.

739. Ziv E, Rotem C, Miodovnik M, et al. (2008) Two modes of ERK activation by TNF in keratinocytes: different cellular outcomes and bi-directional modulation by vitamin $\mathrm{D}$. J Cell Biochem 104, 606-619.

740. Richards JB, Valdes AM, Gardner JP, et al. (2007) Higher serum vitamin D concentrations are associated with longer leukocyte telomere length in women. $A m J$ Clin Nutr 86, 1420-1425.

741. Shea MK, Booth SL, Massaro JM, et al. (2008) Vitamin K and vitamin D status: associations with inflammatory markers in the Framingham Offspring Study. Am J Epidemiol 167, $313-320$.

742. Michos ED, Streeten EA, Ryan KA, et al. (2009) Serum 25-hydroxyvitamin D levels are not associated with subclinical vascular disease or C-reactive protein in the old order amish. Calcif Tissue Int 84, 195-202.

743. Jorde R, Sneve M, Torjesen PA, et al. (2010) No effect of supplementation with cholecalciferol on cytokines and markers of inflammation in overweight and obese subjects. Cytokine 50, 175-180.

744. Hypponen E, Berry D, Cortina-Borja M, et al. (2010) 25-Hydroxyvitamin D and pre-clinical alterations in inflammatory and hemostatic markers: a cross sectional analysis in the 1958 British Birth Cohort. PLoS One 5, e10801.

745. Vilarrasa N, Vendrell J, Maravall J, et al. (2010) Is plasma $25(\mathrm{OH}) \mathrm{D}$ related to adipokines, inflammatory cytokines and insulin resistance in both a healthy and morbidly obese population? Endocrine 38, 235-242.

746. Jablonski KL, Chonchol M, Pierce GL, et al. (2011) 25-Hydroxyvitamin D deficiency is associated with inflammation-linked vascular endothelial dysfunction in middleaged and older adults. Hypertension 57, 63-69.

747. Devaraj S, Yun JM, Duncan-Staley CR, et al. (2011) Low vitamin D levels correlate with the proinflammatory state in type 1 diabetic subjects with and without microvascular complications. Am J Clin Pathol 135, 429-433.

748. Ganji V, Zhang X, Shaikh N, et al. (2011) Serum 25-hydroxyvitamin D concentrations are associated with prevalence of metabolic syndrome and various cardiometabolic risk factors in US children and adolescents based on assayadjusted serum 25-hydroxyvitamin D data from NHANES 2001-2006. Am J Clin Nutr 94, 225-233.

749. Pittas AG, Harris SS, Stark PC, et al. (2007) The effects of calcium and vitamin D supplementation on blood glucose and markers of inflammation in nondiabetic adults. Diabetes Care 30, 980-986.

750. Tasinato A, Boscoboinik D, Bartoli GM, et al. (1995) D-alpha-tocopherol inhibition of vascular smooth muscle cell proliferation occurs at physiological concentrations, correlates with protein kinase $\mathrm{C}$ inhibition, and is independent of its antioxidant properties. Proc Natl Acad Sci U S A 92, 12190-12194.

751. Witham MD, Crighton LJ, Gillespie ND, et al. (2010) The effects of vitamin D supplementation on physical function and quality of life in older patients with heart failure: a randomized controlled trial. Circ Heart Fail 3, 195-201.

752. Zittermann A, Frisch S, Berthold HK, et al. (2009) Vitamin D supplementation enhances the beneficial effects of weight loss on cardiovascular disease risk markers. Am J Clin Nutr 89, 1321-1327. 
753. Palmieri VO, Grattagliano I, Portincasa P, et al. (2006) Systemic oxidative alterations are associated with visceral adiposity and liver steatosis in patients with metabolic syndrome. J Nutr 136, 3022-3026.

754. Sargeant LA, Wareham NJ, Bingham S, et al. (2000) Vitamin $\mathrm{C}$ and hyperglycemia in the European Prospective Investigation into Cancer - Norfolk (EPIC-Norfolk) study: a population-based study. Diabetes Care 23, 726-732.

755. Will JC \& Byers T (1996) Does diabetes mellitus increase the requirement for vitamin C? Nutr Rev 54, 193-202.

756. Ford ES, Mokdad AH, Giles WH, et al. (2003) The metabolic syndrome and antioxidant concentrations: findings from the Third National Health and Nutrition Examination Survey. Diabetes 52, 2346-2352.

757. Aasheim ET, Hofso D, Hjelmesaeth J, et al. (2008) Vitamin status in morbidly obese patients: a cross-sectional study. Am J Clin Nutr 87, 362-369.

758. Canoy D, Wareham N, Welch A, et al. (2005) Plasma ascorbic acid concentrations and fat distribution in 19,068 British men and women in the European Prospective Investigation into Cancer and Nutrition Norfolk cohort study. Am J Clin Nutr 82, 1203-1209.

759. Devaraj S, Li D \& Jialal I (1996) The effects of alpha tocopherol supplementation on monocyte function. Decreased lipid oxidation, interleukin 1 beta secretion, and monocyte adhesion to endothelium. J Clin Invest 98, 756-763.

760. Cominacini L, Garbin U, Pasini AF, et al. (1997) Antioxidants inhibit the expression of intercellular cell adhesion molecule-1 and vascular cell adhesion molecule-1 induced by oxidized LDL on human umbilical vein endothelial cells. Free Radic Biol Med 22, 117-127.

761. Devaraj S \& Jialal I (1999) Alpha-tocopherol decreases interleukin-1 beta release from activated human monocytes by inhibition of 5-lipoxygenase. Arterioscler Thromb Vasc Biol 19, 1125-1133.

762. Cooney RV, Franke AA, Harwood PJ, et al. (1993) Gammatocopherol detoxification of nitrogen dioxide: superiority to alpha-tocopherol. Proc Natl Acad Sci U S A 90, 1771-1775.

763. Christen S, Woodall AA, Shigenaga MK, et al. (1997) gamma-Tocopherol traps mutagenic electrophiles such as $\mathrm{NO}(\mathrm{X})$ and complements alpha-tocopherol: physiological implications. Proc Natl Acad Sci U S A 94, 3217-3222.

764. Handelman GJ, Machlin LJ, Fitch K, et al. (1985) Oral alpha-tocopherol supplements decrease plasma gammatocopherol levels in humans. J Nutr 115, 807-813.

765. Sundl I, Resch U, Bergmann AR, et al. (2004) The decrease in gamma-tocopherol in plasma and lipoprotein fractions levels off within two days of vitamin E supplementation. Ann N Y Acad Sci 1031, 378-380.

766. Lodge JK, Ridlington J, Leonard S, et al. (2001) Alpha- and gamma-tocotrienols are metabolized to carboxyethylhydroxychroman derivatives and excreted in human urine. Lipids 36, 43-48.

767. Jiang Q, Christen S, Shigenaga MK, et al. (2001) gammaTocopherol, the major form of vitamin $\mathrm{E}$ in the US diet, deserves more attention. Am J Clin Nutr 74, 714-722.

768. De Oliveira MC, Alonso A, Lee DH, et al. (2011) Dietary micronutrient intakes are associated with markers of inflammation, but not with markers of subclinical atherosclerosis. J Nutr 141, 1508-1515.

769. Wang L, Gaziano JM, Norkus EP, et al. (2008) Associations of plasma carotenoids with risk factors and biomarkers related to cardiovascular disease in middle-aged and older women. Am J Clin Nutr 88, 747-754.
770. Kritchevsky SB, Bush AJ, Pahor M, et al. (2000) Serum carotenoids and markers of inflammation in nonsmokers. Am J Epidemiol 152, 1065-1071.

771. Erlinger TP, Guallar E, Miller ER III, et al. (2001) Relationship between systemic markers of inflammation and serum beta-carotene levels. Arch Intern Med 161, 1903-1908.

772. Aeberli I, Molinari L, Spinas G, et al. (2006) Dietary intakes of fat and antioxidant vitamins are predictors of subclinical inflammation in overweight Swiss children. Am J Clin Nutr 84, 748-755.

773. van Herpen-Broekmans WM, Klopping-Ketelaars IA, Bots ML, et al. (2004) Serum carotenoids and vitamins in relation to markers of endothelial function and inflammation. Eur J Epidemiol 19, 915-921.

774. Suzuki K, Inoue T, Hashimoto S, et al. (2010) Association of serum carotenoids with high molecular weight adiponectin and inflammation markers among Japanese subjects. Clin Chim Acta 411, 1330-1334.

775. Kubota Y, Moriyama Y, Yamagishi K, et al. (2010) Serum vitamin $\mathrm{C}$ concentration and hs-CRP level in middle-aged Japanese men and women. Atherosclerosis 208, 496-500.

776. Mah E, Matos MD, Kawiecki D, et al. (2011) Vitamin C status is related to proinflammatory responses and impaired vascular endothelial function in healthy, college-aged lean and obese men. J Am Diet Assoc 111, 737-743.

777. Jonasson L, Wikby A \& Olsson AG (2003) Low serum betacarotene reflects immune activation in patients with coronary artery disease. Nutr Metab Cardiovasc Dis 13, 120-125.

778. Hozawa A, Jacobs DR Jr, Steffes MW, et al. (2007) Relationships of circulating carotenoid concentrations with several markers of inflammation, oxidative stress, and endothelial dysfunction: the Coronary Artery Risk Development in Young Adults (CARDIA)/Young Adult Longitudinal Trends in Antioxidants (YALTA) study. Clin Chem 53, 447-455.

779. Walston J, Xue Q, Semba RD, et al. (2006) Serum antioxidants, inflammation, and total mortality in older women. Am J Epidemiol 163, 18-26.

780. Helmersson J, Arnlov J, Larsson A, et al. (2009) Low dietary intake of beta-carotene, alpha-tocopherol and ascorbic acid is associated with increased inflammatory and oxidative stress status in a Swedish cohort. Br J Nutr 101, 1775-1782.

781. Carroll MF \& Schade DS (2003) Timing of antioxidant vitamin ingestion alters postprandial proatherogenic serum markers. Circulation 108, 24-31.

782. Scott DA, Poston RN, Wilson RF, et al. (2005) The influence of vitamin C on systemic markers of endothelial and inflammatory cell activation in smokers and non-smokers. Inflamm Res 54, 138-144.

783. Fumeron C, Nguyen-Khoa T, Saltiel C, et al. (2005) Effects of oral vitamin $\mathrm{C}$ supplementation on oxidative stress and inflammation status in haemodialysis patients. Nephrol Dial Transplant 20, 1874-1879.

784. Jacob K, Periago MJ, Bohm V, et al. (2008) Influence of lycopene and vitamin $\mathrm{C}$ from tomato juice on biomarkers of oxidative stress and inflammation. Br J Nutr 99, 137-146.

785. Tahir M, Foley B, Pate G, et al. (2005) Impact of vitamin E and $\mathrm{C}$ supplementation on serum adhesion molecules in chronic degenerative aortic stenosis: a randomized controlled trial. Am Heart J 150, 302-306.

786. Block G, Jensen C, Dietrich M, et al. (2004) Plasma C-reactive protein concentrations in active and passive smokers: influence of antioxidant supplementation. $J \mathrm{Am}$ Coll Nutr 23, 141-147.

787. Tousoulis D, Antoniades C, Tentolouris C, et al. (2003) Effects of combined administration of vitamins $\mathrm{C}$ and $\mathrm{E}$ 
on reactive hyperemia and inflammatory process in chronic smokers. Atherosclerosis 170, 261-267.

788. Devaraj S \& Jialal I (2000) Alpha tocopherol supplementation decreases serum C-reactive protein and monocyte interleukin-6 levels in normal volunteers and type 2 diabetic patients. Free Radic Biol Med 29, 790-792.

789. Dalgard C, Nielsen F, Morrow JD, et al. (2009) Supplementation with orange and blackcurrant juice, but not vitamin E, improves inflammatory markers in patients with peripheral arterial disease. Br J Nutr 101, 263-269.

790. Devaraj S, Leonard S, Traber MG, et al. (2008) gammaTocopherol supplementation alone and in combination with alpha-tocopherol alters biomarkers of oxidative stress and inflammation in subjects with metabolic syndrome. Free Radic Biol Med 44, 1203-1208.

791. Vincent HK, Bourguignon CM, Vincent KR, et al. (2006) Antioxidant supplementation lowers exercise-induced oxidative stress in young overweight adults. Obesity (Silver Spring) 14, 2224-2235.

792. Wu JH, Ward NC, Indrawan AP, et al. (2007) Effects of alpha-tocopherol and mixed tocopherol supplementation on markers of oxidative stress and inflammation in type 2 diabetes. Clin Chem 53, 511-519.

793. Leichtle A, Teupser D \& Thiery J (2006) Alpha-tocopherol distribution in lipoproteins and anti-inflammatory effects differ between CHD-patients and healthy subjects. $J$ Am Coll Nutr 25, 420-428.

794. Bruunsgaard H, Poulsen HE, Pedersen BK, et al. (2003) Long-term combined supplementations with alpha-tocopherol and vitamin $\mathrm{C}$ have no detectable anti-inflammatory effects in healthy men. $J$ Nutr $\mathbf{1 3 3}, 1170-1173$.

795. Engler MM, Engler MB, Malloy MJ, et al. (2003) Antioxidant vitamins $\mathrm{C}$ and $\mathrm{E}$ improve endothelial function in children with hyperlipidemia: Endothelial Assessment of Risk from Lipids in Youth (EARLY) Trial. Circulation 108, 1059-1063.

796. Mastaloudis A, Morrow JD, Hopkins DW, et al. (2004) Antioxidant supplementation prevents exercise-induced lipid peroxidation, but not inflammation, in ultramarathon runners. Free Radic Biol Med 36, 1329-1341.

797. Rizzo MR, Abbatecola AM, Barbieri M, et al. (2008) Evidence for anti-inflammatory effects of combined administration of vitamin $\mathrm{E}$ and $\mathrm{C}$ in older persons with impaired fasting glucose: impact on insulin action. J Am Coll Nutr 27, 505-511.

798. Rytter E, Vessby B, Asgard R, et al. (2010) Supplementation with a combination of antioxidants does not affect glycaemic control, oxidative stress or inflammation in type 2 diabetes subjects. Free Radic Res 44, 1445-1453.

799. Denniss SG, Haffner TD, Kroetsch JT, et al. (2008) Effect of short-term lycopene supplementation and postprandial dyslipidemia on plasma antioxidants and biomarkers of endothelial health in young, healthy individuals. Vasc Health Risk Manag 4, 213-222.

800. Belisle SE, Leka LS, Delgado-Lista J, et al. (2009) Polymorphisms at cytokine genes may determine the effect of vitamin $\mathrm{E}$ on cytokine production in the elderly. $J$ Nutr 139, 1855-1860.

801. Song Y, Manson JE, Buring JE, et al. (2005) Associations of dietary flavonoids with risk of type 2 diabetes, and markers of insulin resistance and systemic inflammation in women: a prospective study and cross-sectional analysis. $\mathrm{J} \mathrm{Am} \mathrm{Coll}$ Nutr 24, 376-384.

802. Landberg R, Sun Q, Rimm EB, et al. (2011) Selected dietary flavonoids are associated with markers of inflammation and endothelial dysfunction in U.S. women. J Nutr 141, $618-625$.
803. Naruszewicz M, Laniewska I, Millo B, et al. (2007) Combination therapy of statin with flavonoids rich extract from chokeberry fruits enhanced reduction in cardiovascular risk markers in patients after myocardial infraction (MI). Atherosclerosis 194, e179-e184.

804. Suomela JP, Ahotupa M, Yang B, et al. (2006) Absorption of flavonols derived from sea buckthorn (Hippophae rhamnoides L.) and their effect on emerging risk factors for cardiovascular disease in humans. J Agric Food Chem 54, 7364-7369.

805. Egert S, Wolffram S, Bosy-Westphal A, et al. (2008) Daily quercetin supplementation dose-dependently increases plasma quercetin concentrations in healthy humans. J Nutr 138, 1615-1621.

806. Egert S, Bosy-Westphal A, Seiberl J, et al. (2009) Quercetin reduces systolic blood pressure and plasma oxidised low-density lipoprotein concentrations in overweight subjects with a high-cardiovascular disease risk phenotype: a double-blinded, placebo-controlled cross-over study. Br J Nutr 102, 1065-1074.

807. Nieman DC, Henson DA, Davis JM, et al. (2007) Quercetin ingestion does not alter cytokine changes in athletes competing in the Western States Endurance Run. J Interferon Cytokine Res 27, 1003-1011.

808. Nieman DC, Henson DA, Davis JM, et al. (2007) Quercetin's influence on exercise-induced changes in plasma cytokines and muscle and leukocyte cytokine mRNA. J Appl Physiol 103, 1728-1735.

809. McAnulty SR, McAnulty LS, Nieman DC, et al. (2008) Chronic quercetin ingestion and exercise-induced oxidative damage and inflammation. Appl Physiol Nutr Metab 33, $254-262$.

810. D'Anna R, Baviera G, Corrado F, et al. (2005) The effect of the phytoestrogen genistein and hormone replacement therapy on homocysteine and C-reactive protein level in postmenopausal women. Acta Obstet Gynecol Scand 84, 474-477.

811. Yildiz MF, Kumru S, Godekmerdan A, et al. (2005) Effects of raloxifene, hormone therapy, and soy isoflavone on serum high-sensitive C-reactive protein in postmenopausal women. Int J Gynaecol Obstet 90, 128-133.

812. Aubertin-Leheudre M, Lord C, Khalil A, et al. (2007) Effect of 6 months of exercise and isoflavone supplementation on clinical cardiovascular risk factors in obese postmenopausal women: a randomized, double-blind study. Menopause 14, 624-629.

813. Hall WL, Vafeiadou K, Hallund J, et al. (2005) Soyisoflavone-enriched foods and inflammatory biomarkers of cardiovascular disease risk in postmenopausal women: interactions with genotype and equol production. $A m J$ Clin Nutr 82, 1260-1268.

814. Nikander E, Metsa-Heikkila M, Tiitinen A, et al. (2003) Evidence of a lack of effect of a phytoestrogen regimen on the levels of C-reactive protein, E-selectin, and nitrate in postmenopausal women. J Clin Endocrinol Metab 88, $5180-5185$.

815. Steinberg FM, Guthrie NL, Villablanca AC, et al. (2003) Soy protein with isoflavones has favorable effects on endothelial function that are independent of lipid and antioxidant effects in healthy postmenopausal women. Am J Clin Nutr 78, 123-130.

816. Clerici C, Setchell KD, Battezzati PM, et al. (2007) Pasta naturally enriched with isoflavone aglycons from soy germ reduces serum lipids and improves markers of cardiovascular risk. J Nutr 137, 2270-2278. 
817. Hallund J, Tetens I, Bugel S, et al. (2008) The effect of a lignan complex isolated from flaxseed on inflammation markers in healthy postmenopausal women. Nutr Metab Cardiovasc Dis 18, 497-502.

818. Pan A, Demark-Wahnefried W, Ye X, et al. (2009) Effects of a flaxseed-derived lignan supplement on C-reactive protein, IL- 6 and retinol-binding protein 4 in type 2 diabetic patients. Br J Nutr 101, 1145-1149.

819. Pellegrini N, Valtuena S, Ardigo D, et al. (2010) Intake of the plant lignans matairesinol, secoisolariciresinol, pinoresinol, and lariciresinol in relation to vascular inflammation and endothelial dysfunction in middle age-elderly men and post-menopausal women living in Northern Italy. Nutr Metab Cardiovasc Dis 20, 64-71.

820. Salminen S, Bouley C, Boutron-Ruault MC, et al. (1998) Functional food science and gastrointestinal physiology and function. Br J Nutr 80, Suppl. 1, S147-S171.

821. Nova E, Warnberg J, Gomez-Martinez S, et al. (2007) Immunomodulatory effects of probiotics in different stages of life. Br J Nutr 98, Suppl. 1, S90-S95.

822. Ljungh A \& Wadstrom T (2006) Lactic acid bacteria as probiotics. Curr Issues Intest Microbiol 7, 73-89.

823. Sartor RB (2008) Microbial influences in inflammatory bowel diseases. Gastroenterology 134, 577-594.

824. Ley RE, Backhed F, Turnbaugh P, et al. (2005) Obesity alters gut microbial ecology. Proc Natl Acad Sci U S A $\mathbf{1 0 2}$ 11070-11075.

825. Tennyson CA \& Friedman G (2008) Microecology, obesity, and probiotics. Curr Opin Endocrinol Diabetes Obes 15 422-427.

826. Rescigno M (2008) The pathogenic role of intestinal flora in IBD and colon cancer. Curr Drug Targets 9, 395-403.

827. Lomax AR \& Calder PC (2009) Probiotics, immune function, infection and inflammation: a review of the evidence from studies conducted in humans. Curr Pharm Des 15, 1428-1518.

828. De Simone C, Ciardi A, Grassi A, et al. (1992) Effect of Bifidobacterium bifidum and Lactobacillus acidophilus on gut mucosa and peripheral blood B lymphocytes. Immunopharmacol Immunotoxicol 14, 331-340.

829. Collins JK, Dunne C, Murphy L, et al. (2002) A randomized controlled trial of a probiotic Lactobacillus strain in healthy adults: assessment of its delivery, transit and influence on microbial flora and enteric immunity. Micro Ecol Health Dis 81-89.

830. Olivares M, Díaz-Ropero MP, Gomez N, et al. (2006) The consumption of two new probiotic strains Lactobacillus gasseri CECT 5714 and Lactobacillus coryniformis CECT 5711 , boosts the immune system of healthy humans. Int Microbiol 9, 47-52.

831. Olivares M, Paz Diaz-Ropero M, Gomez N, et al. (2006) Dietary deprivation of fermented foods causes a fall in innate immune response. Lactic acid bacteria can counteract the immunological effect of this deprivation. $J$ Dairy Res 73, 492-498.

832. Kekkonen RA, Lummela N, Karjalainen H, et al. (2008) Probiotic intervention has strain-specific anti-inflammatory effects in healthy adults. World $J$ Gastroenterol 14, 2029-2036.

833. Ouwehand AC, Tiihonen K, Saarinen M, et al. (2009) Influence of a combination of Lactobacillus acidophilus NCFM and lactitol on healthy elderly: intestinal and immune parameters. Br J Nutr 101, 367-375.

834. Gibson GR, Probert HM, Loo JV, et al. (2004) Dietary modulation of the human colonic microbiota: updating the concept of prebiotics. Nutr Res Rev 17, 259-275.

835. Food and Agricultural Organisation (2007) FAO Technical Meeting Report: Prebiotics. http://www.fao.org/ ag/agn/ agns/files/Prebiotics_Tech_Meeting_Report.pdf

836. Cani PD, Neyrinck AM, Fava F, et al. (2007) Selective increases of bifidobacteria in gut microflora improve high-fat-diet-induced diabetes in mice through a mechanism associated with endotoxaemia. Diabetologia 50, 2374-2383

837. Guigoz Y, Rochat F, Perruisseau-Carrier G, et al. (2002) Effects of oligosaccharide on the faecal flora and nonspecific immune system in elderly people. Nutr Res (New York, NY) 22, 13-25.

838. Schiffrin EJ, Thomas DR, Kumar VB, et al. (2007) Systemic inflammatory markers in older persons: the effect of oral nutritional supplementation with prebiotics. $J$ Nutr Health Aging 11, 475-479.

839. Lomax AR \& Calder PC (2009) Prebiotics, immune function, infection and inflammation: a review of the evidence. Br J Nutr 101, 633-658.

840. Haussinger D \& Lang F (1991) Cell volume in the regulation of hepatic function: a mechanism for metabolic control. Biochim Biophys Acta 1071, 331-350.

841. Lang F, Busch GL, Ritter M, et al. (1998) Functional significance of cell volume regulatory mechanisms. Physiol Rev 78, 247-306. 